\title{
IMPROVING RECEIVER STATION-KEEPING IN AERIAL REFUELING BY FORMULATING TANKER MOTION AS DISTURBANCE
}

\author{
by \\ CHRISTOPHER MICHAEL ELLIOTT
}

Presented to the Faculty of the Graduate School of

The University of Texas at Arlington in Partial Fulfillment

of the Requirements

for the Degree of

MASTER OF SCIENCE IN AEROSPACE ENGINEERING

THE UNIVERSITY OF TEXAS AT ARLINGTON

December 2009 
Copyright (c) by Christopher Michael Elliott 2009

All Rights Reserved 
To Kelley, Zachary, Shannon, Avery, Brandon, and Kaylin. 


\section{ACKNOWLEDGEMENTS}

I express gratitude to Dr. Dogan for the opportunity to conduct this interesting research on autonomous aerial refueling and for enthusiastically conducting tireless evening discussions to accommodate a busy schedule of a full time engineer, part time distance (remote) education graduate student, and father of five. Thank you to my wife, Kelley, for her unwavering support of a demanding career in conjunction with an intense graduate school schedule. I am grateful to the Distance Education program at the University of Texas at Arlington (UTA), which provided the avenue to continue my Aerospace Engineering college studies in conjunction with a full time position in industry. I thank my company, Lockheed Martin Aeronautics, for encouraging higher education amongst its team members by providing tuition assistance and promoting professional membership participation (e.g. AIAA). Thank you to Mark Tibbs, a colleague at Lockheed Martin (LM), whom has taught among many things, that in an analogous sense, one must walk first by understanding and gaining insight into a low order simplified linear approximation before running blindly into the often cumbersome full order nonlinear problem. Thank you to my other colleagues and friends at LM for providing years of lessons and for providing letters of recommendation to assist my admittance into UTA graduate school (Ken Dorsett, Bob Eller, Dr. Mark Hollingsworth, Dr. Kevin Jawad and many others). Today I appreciate the e-stop red button at one of my early professional positions at the Johnson Space Center, a story in itself, which provided a portion of the fire necessary to burn forward with ambition to complete a MS degree on solely a nocturnal and weekend time allotment. Thank you to Howard and Elnora Cordova for being supportive grandparents, instrumental in my success today. Thank you to my parents, Mike and 
Brenda, for raising a son with encouragement to appreciate math, science, and the art of engineering. The momentum of a helpful lesson from Dad to a confused young student, frustrated and intimidated after a first lesson in basic Algebra to find the solution of $2 x=x+1$, will never cease. Finally, I thank God for all that I have achieved.

November 13, 2009 


\title{
ABSTRACT \\ IMPROVING RECEIVER STATION-KEEPING IN AERIAL REFUELING BY FORMULATING TANKER MOTION AS DISTURBANCE
}

\author{
Christopher Michael Elliott, M.S. \\ The University of Texas at Arlington, 2009
}

Supervising Professor: Atilla Dogan

During aerial refueling operations, the receiver aircraft should hold position within a "refueling box" to maintain the boom-receptacle connection while the tanker aircraft flies in a racetrack maneuver. Prior research work shows, especially in turns, significant difficulty resides in meeting $y$-deviation requirements, i.e., to stay within the box in the lateral direction. This observation indicates tanker motion as "the biggest disturbance" in turn. The nonlinear equations of motion for the receiver aircraft used in this work are developed in terms of position and orientation states relative to the tanker. Linearization results in a set of equations with tanker motion clearly quantified as disturbance. The linearized state-space equation has an additional term with a disturbance matrix representing how tanker motion affects the relative motion. In this research work, a disturbance rejection method, based on these linearized equations, is employed to develop a new control law to reduce the effect of the tanker turning maneuver on the stationkeeping performance of the receiver. This would lead to proactive (feed-forward) control action in addition to reactive (feedback) control. Practical implementation of this approach will require the communication of tanker states (velocity, attitude, angular rates, 
etc.) to the receiver controller. Research findings conclude the new control law offers significant potential to improve the receiver station-keeping performance in the presence of a disturbance where the improvement potential appears directly proportional to the fidelity of the disturbance model. 


\section{TABLE OF CONTENTS}

ACKNOWLEDGEMENTS ............................. iv

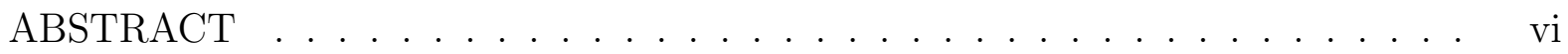

LIST OF FIGURES . . . . . . . . . . . . . . . . . . . . xi

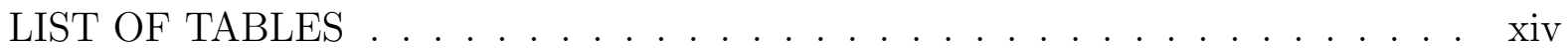

Chapter Page

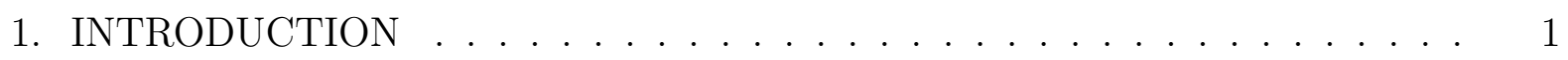

1.1 Background on Aerial Refueling Related Research . . . . . . . . . . . . . 1

1.2 Contribution . . . . . . . . . . . . . . . . . . 4

1.3 Thesis Summary . . . . . . . . . . . . . . . . . 4

2. DETAILED PROBLEM DESCRIPTION . . . . . . . . . . . . . 5

2.1 Translational Kinematics . . . . . . . . . . . . . . . . . . 6

2.1.1 Receiver ..................... 6

2.1 .2 Tanker ........................... 7

2.2 Translational Dynamics . . . . . . . . . . . . . . . . 8

2.2.1 Receiver ..................... 8

2.2.2 Tanker ............................ 9

2.3 Rotational Kinematics . . . . . . . . . . . . . . . . . 10

2.3.1 Receiver .......................... 10

2.3.2 Tanker ......................... 10

2.4 Rotational Dynamics . . . . . . . . . . . . . . . . . . 10

2.4 Receiver ...................... 10

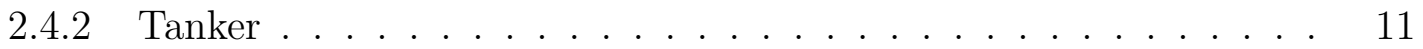


2.5 Linearization . . . . . . . . . . . . . . . . . . . . 11

2.6 Friedland Disturbance Rejection Method . . . . . . . . . . . . . . 13

3. CONTROL SCHEME . . . . . . . . . . . . . . . . . . . . 17

3.1 Tanker . . . . . . . . . . . . . . . . . . . . . . . . 17

3.2 Receiver . . . . . . . . . . . . . . . . . . . . . 20

4. TRANSFORMATION FROM TANKER STATE VECTOR TO

RECEIVER DISTURBANCE VECTOR . . . . . . . . . . . . . . 25

5. SIMULATION RESULTS . . . . . . . . . . . . . . . . . . . 37

5.1 Linear Model . . . . . . . . . . . . . . . . . . . . . . . . 37

5.2 Nonlinear Model . . . . . . . . . . . . . . . . . . . . . . . 55

5.2.1 Control Law Analysis in the Presence of Tanker Flow

Field . . . . . . . . . . . . . . . . . . 56

5.2.2 Control Law Analysis in the Presence of Tanker Flow Field and Non-Steady Prevailing Wind . . . . . . . . . . . . . . . 75

6. SUMMARY, CONCLUSIONS, AND RECOMMENDATIONS FOR FUTURE

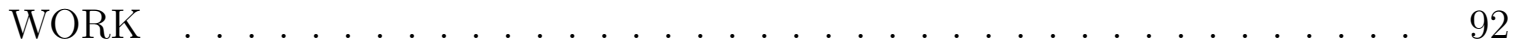

6.1 Summary . . . . . . . . . . . . . . . . . . . . . . . . . 92

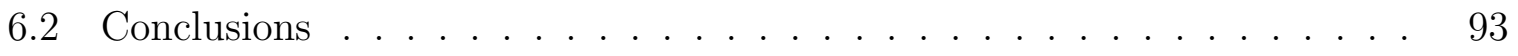

6.3 Future Work . . . . . . . . . . . . . . . . . . . . . . . . . . 93

Appendix

A. EXPANDED SCALAR EQUATIONS OF MOTION FOR RECEIVER AIRCRAFT IN THE ABSENCE OF WINDS $\ldots \ldots \ldots . . \ldots . . . . .98$

B. EXPANDED SCALAR EQUATIONS OF MOTION FOR TANKER AIRCRAFT IN THE ABSENCE OF WINDS . . . . . . . . . . . . . . . . . . . . 103

C. CONTINUOUS TIME LINEAR QUADRATIC REGULATOR SUBOPTIMAL

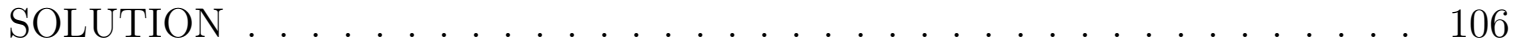

D. TRANSFORMATION OF RECEIVER DISTURBANCE STATE-SPACE MA-

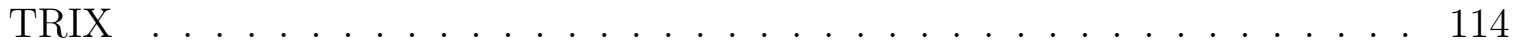

E. SIMULATION RESULTS REPEATED AT OBSERVATION RELATIVE RE- 
FUELING POSITION . . . . . . . . . . . . . . . . . . . 123

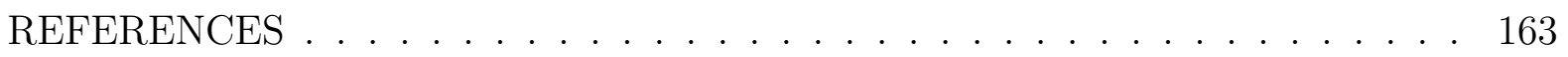

BIOGRAPHICAL STATEMENT . . . . . . . . . . . . . . . . . 167 


\section{LIST OF FIGURES}

Figure $\quad$ Page

4.1 Receiver Open Loop LTI State-Space System . . . . . . . . . . . . . . . . 36

5.1 Receiver Closed Loop LTI State-Space System . . . . . . . . . . . . . . . 38

5.2 Augmented Receiver Closed Loop LTI Metastate-Space System . . . . . . 39

5.3 Receiver Lateral (y) Deviation Step Response (Unity Weight

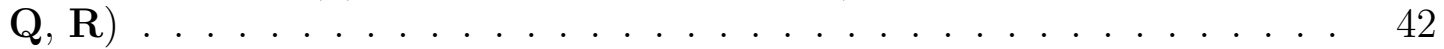

5.4 Receiver Lateral (y) Deviation Frequency Response (Unity Weight

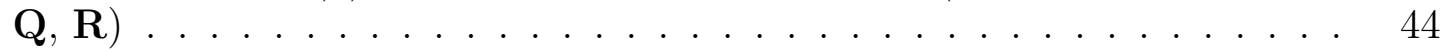

5.5 Receiver Lateral (y) Deviation Step Response (Currently Employed Q, R . . . . . . . . . . . . . . . . . . . 47

5.6 Receiver Lateral (y) Deviation Frequency Response (Currently Employed $\mathbf{Q}, \mathbf{R}) \ldots \ldots \ldots \ldots \ldots \ldots$

5.7 Receiver Lateral (y) Deviation Step Response (Revised Weight

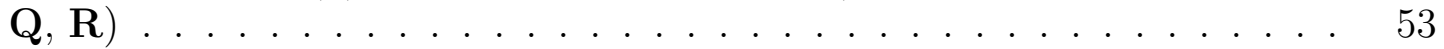

5.8 Receiver Lateral (y) Deviation Frequency Response (Revised Weight

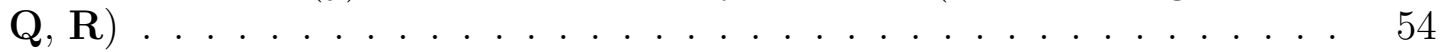

5.9 Receiver Closed Loop Nonlinear System _ . . . . . . . . . . . . . . . . 55

5.10 Nonlinear Simulation Tanker Performing a 30 Degree Bank . . . . . . . . . 56

5.11 Unity Weight Q, R: Station-Keeping and Effectors (Interactive Flow Field Only) with LQR MIMO . . . . . . . . . . . . . . . . 59

5.12 Unity Weight $\mathbf{Q}, \mathbf{R}: V, \beta, \alpha$, and Winds (Interactive Flow Field Only) with $\mathrm{LQR} \mathrm{MIMO} \ldots \ldots \ldots \ldots \ldots$

5.13 Unity Weight Q, R: Station-Keeping and Effectors (Interactive Flow Field Only) with LQR MIMO + Friedland . . . . . . . . . . . . . . . .

5.14 Unity Weight $\mathbf{Q}, \mathbf{R}: V, \beta, \alpha$, and Winds (Interactive Flow Field Only) with LQR MIMO + Friedland . . . . . . . . . . . . . . . .

5.15 Currently Employed Q, R: Station-Keeping and Effectors (Interactive Flow 
Field Only) with LQR MIMO . . . . . . . . . . . . . . . . 64

5.16 Currently Employed Q, R: $V, \beta, \alpha$, and Winds (Interactive Flow Field Only) with LQR MIMO . . . . . . . . . . . . . . . .

5.17 Currently Employed Q, R: Station-Keeping and Effectors (Interactive Flow Field Only) with LQR MIMO + Friedland . . . . . . . . . . . 66

5.18 Currently Employed Q, R: $V, \beta, \alpha$, and Winds (Interactive Flow Field Only) with LQR MIMO + Friedland $\ldots \ldots \ldots \ldots \ldots$

5.19 Currently Employed Q, R: Phase Plane Controller Comparison (Interactive Flow Field Only $\ldots \ldots \ldots \ldots \ldots \ldots$

5.20 Revised Weight Q, R: Station-Keeping and Effectors (Interactive Flow Field Only) with LQR MIMO . . . . . . . . . . . . 70

5.21 Revised Weight $\mathbf{Q}, \mathbf{R}: V, \beta, \alpha$, and Winds (Interactive Flow Field Only) with LQR MIMO . . . . . . . . . . . . . . . . . . 71

5.22 Revised Weight Q, R: Station-Keeping and Effectors (Interactive Flow Field Only) with LQR MIMO + Friedland . . . . . . . . . . . . . 72

5.23 Revised Weight Q, R: $V, \beta, \alpha$, and Winds (Interactive Flow Field Only) with LQR MIMO + Friedland $\ldots \ldots \ldots \ldots \ldots$

5.24 Revised Weight Q, R: Phase Plane LQR MIMO + Friedland Only (Interactive Flow Field Only $\ldots \ldots \ldots \ldots \ldots$

5.25 Nonlinear Simulation Tanker Performing a 15 Degree Right Bank in NonSteady Atmosphere . . . . . . . . . . . . . . . 76

5.26 Nonlinear Simulation Tanker Performing a -15 Degree Left Bank in NonSteady Atmosphere . . . . . . . . . . . . . . . . . 77

5.27 Currently Employed Q, R: Station-Keeping and Effectors (Non-Steady Atmosphere) with LQR MIMO in Right Turn . . . . . . . . . . . . .

5.28 Currently Employed $\mathbf{Q}, \mathbf{R}: V, \beta, \alpha$, and Winds (Non-Steady Atmosphere) with LQR MIMO in Right Turn $\ldots \ldots \ldots \ldots \ldots$

5.29 Currently Employed Q, R: Station-Keeping and Effectors (Non-Steady Atmosphere) with LQR MIMO + Friedland in Right Turn . . . . . . . .

5.30 Currently Employed $\mathbf{Q}, \mathbf{R}: V, \beta, \alpha$, and Winds (Non-Steady Atmosphere) with LQR MIMO + Friedland in Right Turn . . . . . . . . . . .

5.31 Currently Employed Q, R: Gaussian PDF Controller Comparison (Non- 
Steady Atmosphere) in Right Turn . . . . . . . . . . . . . . .

5.32 Currently Employed Q, R: Station-Keeping and Effectors (Non-Steady Atmosphere) with LQR MIMO in Left Turn . . . . . . . . . . . . . .

5.33 Currently Employed Q, R: $V, \beta, \alpha$, and Winds (Non-Steady Atmosphere)

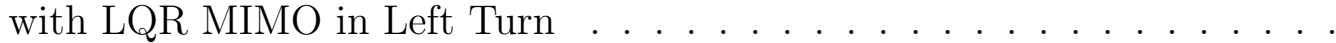

5.34 Currently Employed Q, R: Station-Keeping and Effectors (Non-Steady Atmosphere) with LQR MIMO + Friedland in Left Turn . . . . . . . . . .

5.35 Currently Employed Q, R: $V, \beta, \alpha$, and Winds (Non-Steady Atmosphere) with LQR MIMO + Friedland in Left Turn . . . . . . . . . . . . .

5.36 Currently Employed Q, R: Gaussian PDF LQR MIMO + Friedland Only (Non-Steady Atmosphere) in Left Turn . . . . . . . . . . . . . . . . 


\section{LIST OF TABLES}

Table $\quad$ Page

5.1 Nominal Conditions by Turn Rate and Airspeed of the Tanker . . . . . . . 39

6.1 Summary of Results for Nonlinear Simulation with Receiver at Contact Position . . . . . . . . . . . . . . . . . . . . 92 


\section{CHAPTER 1}

\section{INTRODUCTION}

\subsection{Background on Aerial Refueling Related Research}

While the automation of AR (Aerial Refueling) is disputably a welcomed development in manned air vehicle applications, there is little argument, if any, that this capability is highly desirable for unmanned air vehicles (UAV). Man in the loop AR can pose to be a heavy task workload for both the pilot and fuel boom operator, with a number of variables weighting the complexity and uniqueness of the specific operation. Handling qualities of the receiver airframe aside, the visual conditions, flight profile of the tanker, fuel remaining onboard the receiver, and number of wingmen in queue may constitute only a few stress factors on the pilot's mind during operation. Many of these stressors are potentially minimized, if not alleviated, with an AAR (Automated Aerial Refueling) capability, while the advantage to the UAV application is an obvious increase in endurance time, allowing for a vehicle to maintain theater presence for prolonged durations [1]. Additionally, in flight refueling offered by AAR allows an unmanned vehicle to augment take-off gross weight and refuel once airborne after establishing flight either (i) in a shorter runway distance or (ii) with a valuable payload and cargo weight substitution for initial fuel [2].

Two types of aerial refueling technologies exist in service today. One, the probe and drogue refueling (PDR) method, typically exercised by the US Navy and North Atlantic Treaty Organization (NATO) nations, involves a flexible refueling hose with a wind sock or cone serving as a stabilizing target for the receiver to plug $[1,2]$. The hose and drogue is deployed directly behind the tanker vehicle and basically drug down wake, 
with only the physics of the system serving as stabilization of the refuel point. When not in refueling mode, the PDR hose and drogue is reeled into the body of the tanker and out of sight [2]. The second refueling method is known as boom and receptacle refueling (BRR) and is exercised by the US Air Force (USAF) [1,2]. The BRR method utilizes a rigid boom to transfer fuel from the tanker to the receiver vehicle. For stabilization and active control by a boom operator mounted in the tanker vehicle, the tip of the boom is actuated with control surfaces known as ruddevators [2]. When not in refueling mode, the boom may either remain in flight aft of the tanker vehicle or rotate to a fixed storage position external to the airframe in order to minimize induced drag [2]. A few of the immediate advantages of the BRR method are: the boom operator can help the receiver pilot plug the refueling port where in PDR the pilot is solely responsible for tracking the refueling position at the drogue; maximum fuel transfer rates are significantly higher than PDR transfer rates on the order of 6000 pounds of fuel per minute for BRR compared to only 1500 pounds of fuel per minute with PDR; and the BRR refueling port is typically aft of the critical forward plane of the aircraft (at times directly aft of the pilot station) while the PDR refueling port resides in dangerous proximity to the receiver engine inlet and nose of the vehicle body (which may harness critical pitot static and angle of attack sensors) $[2]$.

A number of research efforts have taken place within academia and the aerospace industry on the automation of aerial refueling. Dr. Atilla Dogan, with the Aerospace Engineering Department of the University of Texas at Arlington, has focused on the development of AAR for the specific BRR refueling method, although many of the concepts are expandable to the PDR method as well. Ref. [3] summarizes the findings in this research to quantify the tanker acceleration in a disturbance model in order to enable control law compensation. Ref. [4] performs a trade study on multiple conceptual vehicles for the AAR task. Ref. [5-8] derives the receiver equations of motion with respect to 
a non-inertial frame. The non-inertial frame considered is the accelerating tanker body, which facilitates an intuitive control approach to tracking relative position between the receiver and the tanker. Interested readers are encouraged to reference these works as the equations of motion derived also allow consideration of time varying mass in the receiver vehicle as the fuel state changes during the AAR task. Ref. [1,9,10] establishes the baseline inner loop linear quadratic regulator and outer loop tracker architecture for controlling relative position in AAR while the tanker vehicle performs a racetrack inertial profile consisting of straight legs and turning legs. Note the term baseline denotes the existing control laws in place targeted for improvement by this thesis research effort. Ref. [11-19] entails the substantial work accomplished on modeling the aerodynamic coupling between two vehicles flying in close proximity and encapsulates the dynamic flow field effects of the tanker down wash onto the receiver vehicle.

While this research work focuses on employing a disturbance rejection methodology described in detail by B. Friedland in Ref. [20], the problem of designing this type of control law has been studied in detail in multiple sources. Early progress in the topic was pioneered by C. Johnson in Ref. [21] which investigated Disturbance Accommodation Control (DAC) for linear time invariant systems by using descriptive waveform models to predict and counteract external effects. Extending the analytical technique into the frequency domain, the authors in Ref. [22] present a similar design method to Johnson's for a linear time invariant system with the use of a different specific waveform model for estimating the disturbance dynamics. More recent work by Johnson in Ref. [23] expands the methodology into Adaptive (or Active) Disturbance Accommodation Control (ADAC). Other modern day research efforts in Ref. [24,25] include nonlinear compensation and emphasize minimizing the control law error (as with classical control) with less dependency on an accurate disturbance model. 


\subsection{Contribution}

The purpose of this research is to improve the AAR control law in place governing the nature of the receiver lateral transient response with respect to the station-keeping BRR refueling position due to tanker flight path heading deviations. Particularly, when the tanker initiates and performs a turn by capturing a bank attitude and maintaining altitude (steady-level turn), the refueling position accelerates away from the receiver. The specific objective undertaken here is to modify the receiver control law sufficiently in order to maintain adequate tracking performance on the refueling position (within half of a meter on all axes), despite the motion of the tanker. With state availability on the tanker flight path heading profile to be commanded, these changes in the target profile are shown to be essentially disturbance inputs in the open loop receiver state-space equation and are used as a feed-forward control law to minimize station-keeping position error.

\subsection{Thesis Summary}

Following a detailed problem description in Chapter 2, a disturbance rejection technique [20] is referenced. Next, Chapter 3 presents the existing control scheme for the tanker and receiver vehicle, which leads to the required steps taken to modify the baseline receiver control algorithm in order to entertain the new disturbance rejection approach in Chapter 4. Subsequently, simulation results are presented in Chapter 5 followed by a final summary, conclusion, and future work recommendation in Chapter 6. Additionally, supporting data, equations, and simulation results are included at the appendices of the thesis. 


\section{CHAPTER 2}

\section{DETAILED PROBLEM DESCRIPTION}

This chapter includes presentation of the matrix-form set of nonlinear, Six Degree of Freedom (6-DOF), rigid body Equations Of Motion (EOM) developed in earlier research [5-8], followed by a detailed reference of the disturbance rejection technique [20] to be employed as a candidate improvement to the existing control law and simulation. In order to realize the EOM for both the receiver and tanker air vehicles, the set of frames used to describe the bodies requires definition. The inertial frame denotes a fixed, nonaccelerating reference set of coordinate axes conventionally used in EOM definition with the application of Newtonian physics and the second law of motion. A body frame is defined for each vehicle and remains rigidly fixed to a reference location and attitude on the airframe. For example, the receiver body frame is fixed in orientation to the fuselage and wing and may be used to describe the vehicle orientation and position with respect to any other coordinate frame. Likewise, the tanker body frame is also fixed to a reference point and orientation on the vehicle. Finally, a wind frame is defined for each vehicle, in which orientation of the set of coordinate axes is fixed to the velocity vector with respect to the surrounding air, rather than the vehicle body. For example, the wind frame of the aircraft is typically used to define the orientation of the vehicle with respect to motion through the air. In other words, the wind frame provides the means to describe the orientation of the vehicle body frame with respect to the velocity vector by the angle of attack and angle of sideslip coordinate rotations.

The tanker vehicle EOM are defined in the conventional sense, with respect to an inertial frame and are provided below in order to allow direct comparison to the receiver 
EOM. The receiver EOM, on the other hand, are defined in a unique manner, with motion often defined relative to an accelerating, non-inertial frame (e.g. tanker body). Refer to Appendix A and Appendix B for the full scalar expansions of the provided matrix equation set, without the wind terms (these components are not used in the control law formulation), for both the receiver vehicle and tanker vehicle, respectively.

\subsection{Translational Kinematics}

\subsubsection{Receiver}

$$
\dot{\xi}=\mathbf{R}_{\mathbf{B}_{\mathbf{R}} \mathbf{B}_{\mathbf{T}}}^{\mathbf{T}} \mathbf{R}_{\mathbf{B}_{\mathbf{R} \mathbf{w}_{\mathbf{R}}}} V_{w}+\mathbf{R}_{\mathbf{B}_{\mathbf{R}} \mathbf{B}_{\mathbf{T}}}^{\mathbf{T}} W_{B_{R}}-\mathbf{R}_{\mathbf{B}_{\mathbf{T}} \mathbf{I}} \dot{r}_{B_{T}}+\mathbf{S}\left(\omega_{\mathbf{B}_{\mathbf{T}}}\right) \xi
$$

where $\xi$ is the representation, in the tanker body frame, of the receiver relative position vector with respect to the tanker vehicle body. The first term on the right side of the equation denotes the velocity vector of the receiver vehicle (with respect to the surrounding air) represented in the receiver wind frame, $\left(V_{w}\right)$, transformed to the tanker vehicle body frame by i) the rotation matrix from receiver wind frame to receiver body frame, $\mathbf{R}_{\mathbf{B}_{\mathbf{R}} \mathbf{w}_{\mathbf{R}}}$, and ii) the rotation matrix from receiver body frame to tanker body frame, $\mathbf{R}_{\mathbf{B}_{\mathbf{R}} \mathbf{B}_{\mathbf{T}}}^{\mathbf{T}}$. Note that the vector $V_{w}=\left[\begin{array}{lll}V & 0 & 0\end{array}\right]^{T}$ where $V$ is the true airspeed of the receiver. The second term includes the atmospheric wind velocity vector with respect to the inertial frame and represented in the receiver vehicle body frame, $W_{B_{R}}$ (and includes flow field effects of tanker downwash) or, in other words, the air fluidic velocity in the neighborhood of the receiver position, represented in the receiver body. This wind term is transformed to the tanker vehicle body frame by the rotation matrix, $\mathbf{R}_{\mathbf{B}_{\mathbf{R}} \mathbf{B}_{\mathbf{T}}}$. The latter two terms on the right hand side of Eq. (2.1) represent the influence of the tanker motion on the receiver relative position to the tanker body. The component with $\dot{r}_{B_{T}}$ is the velocity vector of the tanker with respect to the inertial frame, transformed from 
inertial frame to tanker body with the Euler angle rotation matrix, $\mathbf{R}_{\mathbf{B}_{\mathbf{T}} \mathbf{I}}$. Finally, the remaining term on the right side of the equation describes the impact of tanker angular velocity, $\omega_{B_{T}}$, defined with respect to the inertial frame and represented in the tanker body frame, on the receiver relative motion rate. In other words, the pitch, yaw, and roll rates of the tanker directly contribute to the relative velocity between the two vehicles and that contribution is proportional to the relative position between the two vehicles, $\xi$. Additionally, due to describing the equations in matrix form, the notation $\mathbf{S}(\cdot)$ represents the skew symmetric operation as follows [1]:

$$
\mathbf{S}(x)=\left[\begin{array}{ccc}
0 & x_{3} & -x_{2} \\
-x_{3} & 0 & x_{1} \\
x_{2} & -x_{1} & 0
\end{array}\right],
$$

where $x=\left[\begin{array}{lll}x_{1} & x_{2} & x_{3}\end{array}\right]^{T}$ is the representation of an arbitrary vector $\underline{x}$.

\subsubsection{Tanker}

$$
\dot{r}_{B_{T}}=\mathbf{R}_{\mathbf{B}_{\mathbf{T}} \mathbf{I}}^{\mathbf{T}} \mathbf{R}_{\mathbf{B}_{\mathbf{T}} \mathbf{w}_{\mathbf{T}}} V_{w_{T}}+W
$$

In contrast, due to the conventional definition with respect to a fixed inertial frame, the tanker translational kinematics matrix-form equation includes only the first two terms describing the vehicle velocity, $V_{w_{T}}$, with respect to the surrounding air, and again the wind velocity $W$ (the air fluidic velocity now includes only the atmospheric prevailing wind and inertial turbulence in the neighborhood of the tanker, represented and defined with respect to the inertial frame). Reiterating, $W$ is the representation of winds in the inertial frame while $W_{B_{R}}$ in Eq. (2.1) is the representation of winds in the receiver body frame. 
2.2 Translational Dynamics

\subsubsection{Receiver}

$$
\begin{aligned}
{\left[\begin{array}{c}
\dot{V} \\
\dot{\beta} \\
\dot{\alpha}
\end{array}\right]=} & \mathcal{E}_{\mathbf{R}}^{-\mathbf{1}}\left[\mathbf{S}\left(\omega_{\mathbf{B}_{\mathbf{R}} \mathbf{B}_{\mathbf{T}}}\right)+\mathbf{R}_{\mathbf{B}_{\mathbf{R}} \mathbf{B}_{\mathbf{T}}} \mathbf{S}\left(\omega_{\mathbf{B}_{\mathbf{T}}}\right) \mathbf{R}_{\mathbf{B}_{\mathbf{R}} \mathbf{B}_{\mathbf{T}}}^{\mathbf{T}}\right]\left(\mathbf{R}_{\mathbf{B}_{\mathbf{R}} \mathbf{w}_{\mathbf{R}}} V_{w}+W_{B_{R}}\right) \\
& -\mathcal{E}_{\mathbf{R}}^{-\mathbf{1}} \dot{W}_{B_{R}}+\frac{1}{m_{R}} \mathcal{E}_{\mathbf{R}}^{-\mathbf{1}}\left(\mathbf{R}_{\mathbf{B}_{\mathbf{R}} \mathbf{B}_{\mathbf{T}}} \mathbf{R}_{\mathbf{B}_{\mathbf{T}} \mathbf{I}} M_{R}+\mathbf{R}_{\mathbf{B}_{\mathbf{R}} \mathbf{w}_{\mathbf{R}}} A_{R}+P_{R}\right)
\end{aligned}
$$

where

$$
\mathcal{E}_{\mathbf{R}}^{-1}=\left[\begin{array}{ccc}
\cos \alpha \cos \beta & \sin \beta & \cos \beta \sin \alpha \\
-\frac{1}{V} \cos \alpha \sin \beta & \frac{1}{V} \cos \beta & -\frac{1}{V} \sin \alpha \sin \beta \\
-\frac{1}{V} \sec \beta \sin \alpha & 0 & \frac{1}{V} \cos \alpha \sec \beta
\end{array}\right]
$$

The translational dynamics equation is written in terms of the velocity vector of the receiver aircraft relative to the surrounding air in the neighborhood of the vehicle. Specifically, the true airspeed $V$, angle of sideslip $\beta$, and angle of attack $\alpha$ are used for expressing this vector. Inspecting the right hand side of the equation, there are three basic components contributing to the acceleration: (i) the angular velocity vectors $\left(\omega_{B_{R} B_{T}}\right.$ and $\left.\omega_{B_{T}}\right)$ of the two vehicles coupled with both the receiver velocity $V_{w}$ and the wind velocity $W_{B_{R}}$, (ii) the wind acceleration, $\dot{W}_{B_{R}}$, and (iii) the external forces on the receiver $\left(M_{R}\right.$ gravitational force represented in the inertial frame, $A_{R}$ aerodynamic force represented in the receiver wind frame, and $P_{R}$ propulsive force represented in the receiver body frame). 
2.2.2 Tanker

$$
\begin{aligned}
{\left[\begin{array}{c}
\dot{V}_{T} \\
\dot{\beta}_{T} \\
\dot{\alpha}_{T}
\end{array}\right]=} & \mathcal{E}_{\mathbf{T}}^{-1} \mathbf{S}\left(\omega_{\mathbf{B}_{\mathbf{T}}}\right)\left(\mathbf{R}_{\mathbf{B}_{\mathbf{T}} \mathbf{w}_{\mathbf{T}}} V_{w_{T}}+\mathbf{R}_{\mathbf{B}_{\mathbf{T}} \mathbf{I}} W\right)-\mathcal{E}_{\mathbf{T}}^{-1} \mathbf{R}_{\mathbf{B}_{\mathbf{T}} \mathbf{I}} \dot{W} \\
& +\frac{1}{m_{T}} \mathcal{E}_{\mathbf{T}}^{-1}\left(\mathbf{R}_{\mathbf{B}_{\mathbf{T}} \mathbf{I}} M_{T}+\mathbf{R}_{\mathbf{B}_{\mathbf{T}} \mathbf{w}_{\mathbf{T}}} A_{T}+P_{T}\right)
\end{aligned}
$$

where

$$
\mathcal{E}_{\mathbf{T}}^{-1}=\left[\begin{array}{ccc}
\cos \alpha_{T} \cos \beta_{T} & \sin \beta_{T} & \cos \beta_{T} \sin \alpha_{T} \\
-\frac{1}{V_{T}} \cos \alpha_{T} \sin \beta_{T} & \frac{1}{V_{T}} \cos \beta_{T} & -\frac{1}{V_{T}} \sin \alpha_{T} \sin \beta_{T} \\
-\frac{1}{V_{T}} \sec \beta_{T} \sin \alpha_{T} & 0 & \frac{1}{V_{T}} \cos \alpha_{T} \sec \beta_{T}
\end{array}\right]
$$

Comparison of Eq. (2.6) to Eq. (2.4) reveals that the primary difference resides in the first term on the right hand side of the equations. This difference is due to the fact that the angular velocity of the receiver is defined relative to a non-inertial rotating frame while the tanker angular velocity is written relative to the inertial frame. There are also subtle differences in the components (ii) and (iii). Component (ii) now includes the rotation matrix from inertial frame to tanker body frame, $\mathbf{R}_{\mathbf{B}_{\mathbf{T}} \mathbf{I}}$, due to the wind vector representation in the inertial frame. Although the form of component (iii) in Eq. (2.6) is slightly different, the term remains describing the external forces on the tanker vehicle $\left(M_{T}\right.$ gravitational force represented in the inertial frame, $A_{T}$ aerodynamic force represented in the tanker wind frame, and $P_{T}$ propulsive force represented in the tanker body frame). 


\subsection{Rotational Kinematics}

\subsubsection{Receiver}

$$
\mathbf{R}_{\mathbf{B}_{\mathbf{R}} \mathbf{B}_{T}} \dot{\mathbf{R}}_{\mathbf{B}_{\mathbf{R}} \mathbf{B}_{\mathrm{T}}}^{\mathbf{T}}=-\mathbf{S}\left(\omega_{\mathbf{B}_{\mathrm{R}} \mathbf{B}_{\mathrm{T}}}\right)
$$

Eq. (2.8) governs the attitude rate of the receiver vehicle, defined with respect to the tanker vehicle body, where $\omega_{B_{R} B_{T}}$ denotes the angular velocity of the receiver with respect to the tanker body frame and expressed in the receiver body frame.

\subsubsection{Tanker}

$$
\mathbf{R}_{\mathbf{B}_{\mathbf{T}} \mathbf{I}} \dot{\mathbf{R}}_{\mathbf{B}_{\mathbf{T}} \mathbf{I}}^{\mathbf{T}}=-\mathbf{S}\left(\omega_{\mathbf{B}_{\mathbf{T}}}\right)
$$

The rotational kinematics of the tanker vehicle are defined with respect to the inertial frame in Eq. (2.9). This set of equations in conventional form is the closest resemblance to the new unique set of equations of motion for the receiver, defined with respect to the accelerating tanker vehicle body frame. The sole difference is the relative frame definition.

\subsection{Rotational Dynamics}

\subsubsection{Receiver}

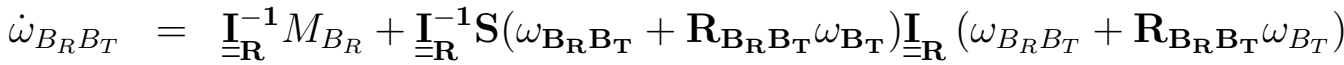

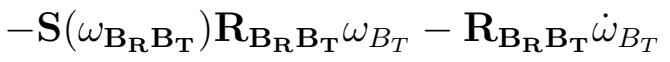

Eq. (2.10) governs the rotational dynamics of the receiver vehicle, where the rotational velocity of the receiver is defined with respect to the tanker body frame and represented 
in the receiver body frame. Four acceleration components constitute the right hand side of the equation. The first two terms are the (i) external moments on the receiver vehicle, $M_{B_{R}}$, taken about the receiver body frame origin, and (ii) the receiver inertia component on total angular velocity between the two rotating vehicles where $\underline{\underline{\mathbf{I}}}_{\mathbf{R}}$ denotes the receiver aircraft inertia matrix. The last two terms, (iii) and (iv), are the effect of the tanker angular velocity and acceleration on the angular acceleration of the receiver relative to the tanker body, respectively.

\subsubsection{Tanker}

$$
\dot{\omega}_{B_{T}}=\underline{\underline{\mathbf{I}}}_{\mathbf{T}}^{-\mathbf{1}} M_{B_{T}}+\underline{\underline{\mathbf{I}}}_{\mathbf{T}}^{-\mathbf{1}} \mathbf{S}\left(\omega_{\mathbf{B}_{\mathbf{T}}}\right) \underline{\underline{\mathbf{I}}}_{\mathbf{T}} \omega_{B_{T}}
$$

The traditional definition of rotational acceleration with respect to an inertial frame is used to define the tanker vehicle in Eq. (2.11). In contrast to Eq. (2.10), the primary difference is the complete absence of the last two components (iii) and (iv) as described in the receiver rotational dynamics. The remaining components are similar only now $M_{B_{T}}$ in (i) denotes external moments on the tanker vehicle about the tanker body frame origin and the angular velocity term in (ii) is strictly the tanker. Finally, $\underline{\mathbf{I}}_{\mathbf{T}}$ denotes the tanker aircraft inertia matrix.

\subsection{Linearization}

Recapitulating, the unconventional terms in the receiver set of equations of motion are due to derivation with respect to a non-inertial frame under the effect of wind exposure. Interestingly, the terms resulting from this approach liquidate to define the motion of the rotating non-inertial frame (tanker body) to the fixed, inertial frame. For example, $\dot{r}_{B_{T}}$ defines the tanker inertial translational kinematics, while the $\omega_{B_{T}}$ and $\mathbf{R}_{\mathbf{B}_{\mathbf{T}} \mathbf{I}}$ compo- 
nents define the tanker angular velocity and inertial orientation, respectively. Linearizing, the receiver equations are represented in the state-space form as

$$
\underline{\dot{x}}=\mathbf{A} \underline{x}+\mathbf{B} \underline{u}+\mathbf{H}(\mathbf{t}) \underline{w}
$$

where $\mathbf{A} \in \Re^{12 x 12}, \mathbf{B} \in \Re^{12 x 6}$, and $\mathbf{H}(\mathbf{t}) \in \Re^{12 x 12}$. Again, breaking from typical convention for a linearized set of equations of motion with respect to an inertial frame, the unique terms governing motion relative to an accelerating tanker body frame are conglomerated into the disturbance or exogenous vector

$$
\underline{w}=\left[\begin{array}{llllllllllll}
\dot{x}_{T} & \dot{y}_{T} & \dot{z}_{T} & p_{T} & q_{T} & r_{T} & \dot{p}_{T} & \dot{q}_{T} & \dot{r}_{T} & \psi_{T} & \theta_{T} & \phi_{T}
\end{array}\right]^{T} \in \Re^{12 x 1}
$$

with the receiver state vector and input vector represented as $\underline{x}$ and $\underline{u}$, respectively, as

$$
\begin{gathered}
\underline{x}=\left[\begin{array}{cccccccccccc}
V & \beta & \alpha & p & q & r & \psi & \theta & \phi & x & y & z
\end{array}\right]^{T} \in \Re^{12 x 1} \\
\underline{u}=\left[\begin{array}{lllllll}
\delta_{a} & \delta_{e} & \delta_{r} & \xi & \delta_{y} & \delta_{z}
\end{array}\right]^{T} \in \Re^{6 x 1}
\end{gathered}
$$

A key point requiring emphasis is that the inclusion of the time variant $\mathbf{H}(\mathbf{t}) \underline{w}$ term in the open loop receiver state-space equation does not yet define a control scheme but represents the governing physics between the two bodies of interest. Closing the loop on this information however, as proposed in detail by Friedland [20], will construct the unique control law in order to improve tracking performance. The existing LQR MIMO $[1,9,10]$ position tracker in place does not employ this technique to utilize a disturbance measurement, and asserts that the approach is not an absolute requirement to achieve a controllable closed loop system. However, the objective for this implementation is to improve the controller transient response in station-keeping position error, primarily with respect to lateral deviation from the target. As observed in previous simulations $[1,5-7,9$, 10], the most prominent lateral deviations encountered with the existing control law are in response to a tanker flight path deviation (i.e. heading rate onset due to a level turn). Although additional disturbance sources on the receiver closed loop response exist such 
as prevailing wind, wake-vortex induced wind, and freestream turbulence [16-19], this research work focuses on improving the performance degradation caused by acceleration and rotation of the tanker. Nevertheless, in the simulations for evaluating the closed loop performance, other disturbances will also be included.

\subsection{Friedland Disturbance Rejection Method}

Consider the linear state-space representation of a system experiencing disturbance as

$$
\underline{\dot{x}}=\mathbf{A} \underline{x}+\mathbf{B} \underline{u}+\mathbf{E} \underline{x}_{T_{A}}
$$

Friedland [20] proposes that if the disturbance vector of the system is described in a first order differential equation of the form,

$$
\underline{\dot{x}}_{T_{A}}=\mathbf{A}_{\mathbf{T}_{\mathbf{C L}}} \underline{x}_{T_{A}}
$$

the exogenous dynamics may be combined with the open loop state-space representation to formulate a set of equations or metastate-space system as

$$
\underline{\dot{x}}_{o}=\mathbf{A}_{\mathbf{o}} \underline{x}_{o}+\mathbf{B}_{\mathbf{o}} \underline{u}
$$

where

$$
\underline{x}_{o}=\left[\frac{\underline{x}}{\underline{x}_{T_{A}}}\right]
$$

represents the adjoined receiver state and disturbance vectors (in this case, the latter as augmented tanker states, $\left.\underline{x}_{T_{A}}\right)$. Metastate matrices $\mathbf{A}_{\mathbf{o}}$ and $\mathbf{B}_{\mathbf{o}}$ represent the original state-space expressed in Eq. (2.14), as well as encapsulation of the exogenous components into the open loop state and control matrices,

$$
\mathbf{A}_{\mathbf{o}}=\left[\begin{array}{c|c}
\mathbf{A} & \mathbf{E} \\
\hline 0 & \mathbf{A}_{\mathbf{T}_{\mathbf{C L}}}
\end{array}\right] \text { and } \mathbf{B}_{\mathbf{o}}=\left[\begin{array}{c}
\mathbf{B} \\
\hline 0
\end{array}\right]
$$


respectively. Precluding further detailed discussion until Chapters 3 and 4, note the appearance of the tanker closed loop dynamics matrix, $\mathbf{A}_{\mathbf{T}_{\mathbf{C L}}}$, in the receiver open loop metastate matrix, which will be of particular value. This value, to be presented, will enable a key step in the problem approach to utilize Eq. (2.14) as opposed to Eq. (2.12), in order to realize the time invariant metastate-space system [20].

Friedland continues description of the solution to the exogenous problem with explanation of two notable considerations for the LQR application. One, the exogenous states are uncontrollable and therefore render zero weighting components in the metastate penalty matrix, Q, within the quadratic performance integral. In other words, considering the cost minimization problem, there is no penalty for the behavior of the external system. Secondly, considering the infinite horizon linear quadratic regulator problem whose performance index takes the form,

$$
V_{\infty}=\int_{0}^{\infty}\left[\underline{x}_{o}^{T}(t) \mathbf{Q} \underline{x}_{o}(t)+\underline{u}^{T}(t) \mathbf{R} \underline{u}(t)\right] d t
$$

also poses the realization that an uncontrollable exogenous state within the $\underline{x}_{o}$ vector may cause the controllable receiver state, $\underline{x}$, to be non-zero as time approaches infinite (e.g. the tanker imposes a disturbance on the receiver which leads to a static error in the state regulator control law). In turn, the cost (performance index) integral approaches infinite due to a non-zero $\underline{x}_{o}^{T}(t) \mathbf{Q} \underline{x}_{o}(t)$ term, and a steady state solution to the LQR problem may fail to exist [20]. Friedland's approach to this problem is to employ a control action, $\underline{u}(t)$, to ensure a zero steady state error (i.e. drive $\underline{x}$ to zero regardless of disturbance). Appropriately, per this proposition, the research work here utilizes an infrastructure with an integral control outer loop structure (as presented ahead in Chapter 3) in conjunction with a suboptimal linear quadratic regulator inner loop. Solving for the suboptimal control law, using the procedure referenced in detail in Appendix C,

$$
\underline{u}(t)=-\mathbf{R}^{-1} \mathbf{B}_{\mathbf{o}}^{T} \overline{\mathbf{M}} \underline{x}_{o}(t)
$$


where $\overline{\mathbf{M}}$ represents the steady state solution to the well known Ricatti differential equation (or algebraic Ricatti equation due to the steady state form) typically encountered in optimal control practice, given by

$$
-\dot{\hat{\mathbf{M}}}=\mathbf{A}_{\mathrm{o}}{ }^{T} \hat{\mathbf{M}}+\hat{\mathbf{M}} \mathbf{A}_{\mathrm{o}}-\hat{\mathbf{M}} \mathbf{B}_{\mathrm{o}} \mathbf{R}^{-1} \mathbf{B}_{\mathrm{o}}{ }^{T} \hat{\mathbf{M}}+\mathbf{Q}=\mathbf{0}
$$

Note the control law in Eq. (2.18) by definition formulates a compensatory action on the receiver alone. In other words, the exogenous system (tanker) is not impacted by the feedback of $\underline{u}(t)$. Therefore, the product of the metastate $\mathbf{B}_{\mathbf{o}}$ input matrix and the control action, $\underline{u}(t)$ is a contribution solely to the receiver state rate $\underline{\dot{x}}$ in Eq. (2.14) and is a vector of compatible length $\in \Re^{12 x 1}$. With this logic in regard, the Ricatti kernel, $\hat{\mathbf{M}}$ in Eq. (2.19) is partitioned in accordance with the metastate-space system construction.

$$
\hat{\mathbf{M}}=\left[\begin{array}{ll}
\hat{\mathbf{M}}_{1} & \hat{\mathbf{M}}_{2} \\
\hat{\mathbf{M}}_{2}^{T} & \hat{\mathbf{M}}_{3}
\end{array}\right]
$$

Continuing expansion of the receiver control law in Eq. (2.18) in accordance with the partitioned Ricatti kernels in Eq. (2.20),

$$
\begin{aligned}
\underline{u}(t) & =-\mathbf{R}^{-1}\left[\begin{array}{ll}
\mathbf{B}^{T} & \mathbf{0}
\end{array}\right]\left[\begin{array}{cc}
\hat{\mathbf{M}}_{1} & \hat{\mathbf{M}}_{2} \\
\hat{\mathbf{M}}_{2}^{T} & \hat{\mathbf{M}}_{\mathbf{3}}
\end{array}\right]\left[\begin{array}{c}
\underline{x} \\
\underline{\underline{x}} \\
\underline{T}_{A}
\end{array}\right] \\
& =-\left[\begin{array}{ll}
\mathbf{R}^{-1} \mathbf{B}^{T} \hat{\mathbf{M}}_{\mathbf{1}} & \mathbf{R}^{-1} \mathbf{B}^{T} \hat{\mathbf{M}}_{\mathbf{2}}
\end{array}\right]\left[\begin{array}{c}
\underline{x} \\
\underline{\underline{x}}
\end{array}\right]
\end{aligned}
$$

Therefore, the suboptimal linear control law is

$$
\underline{u}=-\mathbf{R}^{-1} \mathbf{B}^{\mathrm{T}} \overline{\mathbf{M}}_{1} \underline{x}-\mathbf{R}^{-1} \mathbf{B}^{\mathrm{T}} \overline{\mathbf{M}}_{\mathbf{2}} \underline{x}_{T_{A}}
$$

offering "effective control for a class of exogenous inputs" [20]. Stated alternatively, the control action counteracts the tanker induced disturbances by the factor $\mathbf{R}^{-1} \mathbf{B}^{\mathbf{T}} \overline{\mathbf{M}}_{\mathbf{2}}$ where $\overline{\mathbf{M}}_{1}$ and $\overline{\mathbf{M}}_{2}$ are constant matrices and represent the partitioned kernels in the 
steady state solution to the Ricatti algebraic equation presented above in Eq. (2.19). Therefore, the control law in Eq. (2.22) only requires solving the gain submatrices $\overline{\mathbf{M}}_{\mathbf{1}}$ and $\overline{\mathbf{M}}_{\mathbf{2}}$ in Eq. (2.20), provided by expanding the partitions in Eq. (2.19). Eq. (2.23) below presents the expanded equation governing $\overline{\mathbf{M}}_{\mathbf{1}}$ and retains the general Ricatti algebraic form. (Note $\mathbf{Q}_{x}$ represents the state weight matrix components on $\underline{x}$ alone and does not include the zero penalty terms on the exogenous vector, $\underline{x}_{T_{A}}$ ).

$$
-\dot{\bar{M}}_{1}=\overline{\mathbf{M}}_{1} \mathrm{~A}+\mathrm{A}^{\mathrm{T}} \overline{\mathbf{M}}_{1}-\overline{\mathbf{M}}_{1} \mathrm{BR}^{-1} \mathrm{~B}^{\mathrm{T}} \overline{\mathbf{M}}_{1}+\mathrm{Q}_{x}=0
$$

$\overline{\mathbf{M}}_{2}$ however, requires consideration of a new differential equation (again now in the steady state algebraic form), unique to the exogenous metastate-space system.

$$
-\dot{\bar{M}}_{2}=\overline{\mathbf{M}}_{1} \mathbf{E}+\overline{\mathbf{M}}_{2} \mathbf{A}_{\mathbf{T}_{\mathrm{CL}}}+\left(\mathrm{A}^{\mathbf{T}}-\overline{\mathbf{M}}_{1} \mathrm{BR}^{-1} \mathbf{B}^{\mathbf{T}}\right) \overline{\mathbf{M}}_{2}=\mathbf{0}
$$

The key matrices in Eq. (2.24) that must be known in order to employ the Friedland disturbance rejection method [20] are $\mathbf{E}$ and $\mathbf{A}_{\mathbf{T}_{\mathbf{C L}}}$, also observed in Eq. (2.14) and Eq. (2.15), respectively. In other words, (i) the transformation matrix of the exogenous state vector into the receiver state-space system (E) and (ii) the matrix governing the first order differential equation, $\mathbf{A}_{\mathbf{T}_{\mathbf{C L}}}$, describing the exogenous dynamics both require quantification for this control law. 


\section{CHAPTER 3}

\section{CONTROL SCHEME}

The purpose of this chapter is to serve as a brief reference to the existing control methodology developed in prior work $[1,9,10]$. The linearized equations for the open loop and closed loop vehicle dynamics are provided herein for both the tanker and receiver. Additionally, full state availability for both vehicles is assumed, and all conditions necessary for the convergence of the linear quadratic solution are met. Namely, (i) the state-space matrices (A, B) are controllable; and (ii) the positive semidefinite state penalty matrix, $\mathbf{Q}$, results in the observability of $(\sqrt{\mathbf{Q}}, \mathbf{A})$ in both applications below [26].

\subsection{Tanker}

The open loop linearized state-space system is described per nominal condition $i$ by the following:

$$
\Delta \underline{x}_{T}=\mathbf{A}_{T, i} \Delta \underline{x}_{T}+\mathbf{B}_{T, i} \Delta \underline{u}_{T}
$$

with state vector,

$$
\Delta \underline{x}_{T}=\left[\begin{array}{lllllllllll}
\Delta V_{T} & \Delta \beta_{T} & \Delta \alpha_{T} & \Delta p_{T} & \Delta q_{T} & \Delta r_{T} & \Delta \theta_{T} & \Delta \phi_{T} & \Delta z_{T} & \Delta \psi_{T}
\end{array}\right]^{T} \in \Re^{10 x 1}(3
$$

and control input vector,

$$
\Delta \underline{u}_{T}=\left[\Delta \delta_{a_{T}} \Delta \delta_{e_{T}} \Delta \delta_{r_{T}} \Delta \xi_{t_{T}}\right]^{T}
$$

where index $i$ refers to the $i^{\text {th }}$ nominal condition and conventional effectors of aileron, elevator, rudder, and throttle are denoted with $\delta_{a_{T}}, \delta_{e_{T}}, \delta_{r_{T}}$, and $\xi_{t_{T}}$, respectively. For outer loop control, the output vector is chosen as velocity, altitude, and bank attitude.

$$
\underline{y}_{T}=\left[\begin{array}{lll}
\Delta V_{T} & \Delta z_{T} & \Delta \phi_{T}
\end{array}\right]^{T}
$$


The control error rate (to be utilized for integral control) is defined as the difference between output vector and the desired reference or commanded value as

$$
\underline{\dot{e}}_{T}=\underline{y}_{T}-\underline{y}_{T, c}=\mathbf{C}_{\mathbf{T}, \mathbf{i}} \Delta \underline{x}_{T}-\underline{y}_{T, c}
$$

or in scalar form,

$$
\begin{aligned}
& \dot{e}_{V_{T}}=\Delta V_{T}-\Delta V_{T, c} \\
& \dot{e}_{z_{T}}=\Delta z_{T}-\Delta z_{T, c} \\
& \dot{e}_{\phi_{T}}=\Delta \phi_{T}-\Delta \phi_{T, c}
\end{aligned}
$$

Next, the open loop system state vector is augmented with the errors as follows:

$$
\left[\begin{array}{c}
\Delta \underline{\dot{x}}_{T} \\
\underline{\dot{e}}_{T}
\end{array}\right]=\left[\begin{array}{cc}
\mathbf{A}_{T, i} & \mathbf{0}_{\mathbf{1 0 \times 3}} \\
\mathbf{C}_{\mathbf{T}, \mathbf{i}} & \mathbf{0}_{\mathbf{3} \times \mathbf{3}}
\end{array}\right]\left[\begin{array}{c}
\Delta \underline{x}_{T} \\
\underline{e}_{T}
\end{array}\right]+\left[\begin{array}{c}
\mathbf{B}_{T, i} \\
\mathbf{0}_{\mathbf{3} \times \mathbf{4}}
\end{array}\right] \underline{u}_{T}-\left[\begin{array}{c}
\mathbf{0}_{\mathbf{1 0 \times 3}} \\
\mathbf{I}_{\mathbf{3} \times \mathbf{3}}
\end{array}\right] \underline{y}_{T, c}
$$

The feedback control gain matrix is computed as the solution to the algebraic Ricatti equation (in a similar procedure as discussed in Chapter 2.

$$
\Delta \underline{u}_{T, i}=-\mathbf{K}_{\mathbf{T}, \mathbf{i}}\left[\begin{array}{c}
\Delta \underline{x}_{T} \\
\underline{e}_{T}
\end{array}\right]=-\mathbf{K}_{\mathbf{x}_{\mathbf{T}, \mathbf{i}}} \Delta \underline{x}_{T}-\mathbf{K}_{\mathbf{e}_{\mathbf{T}, \mathbf{i}}} \underline{e}_{T}
$$

The gain, $\mathbf{K}_{\mathbf{T}, \mathbf{i}}$ is then decomposed to allow for inner loop state feedback in conjunction with outer loop integral control on the output vector. Finally, the control law is substituted into the open loop augmented system, yielding the closed loop augmented system as

$$
\left[\begin{array}{c}
\Delta \underline{\dot{x}}_{T} \\
\dot{e}_{T}
\end{array}\right]=\left[\begin{array}{ll}
\left(\mathbf{A}_{T, i}-\mathbf{B}_{T, i} \mathbf{K}_{\mathbf{x}_{\mathbf{T}, \mathbf{i}}}\right) & -\mathbf{B}_{T, i} \mathbf{K}_{\mathbf{e}_{\mathbf{T}, \mathbf{i}}} \\
\mathbf{C}_{\mathbf{T}, \mathbf{i}} & \mathbf{0}_{\mathbf{3} \times \mathbf{3}}
\end{array}\right]\left[\begin{array}{c}
\Delta_{\underline{x}_{T}} \\
\underline{e}_{T}
\end{array}\right]-\left[\begin{array}{c}
\mathbf{0}_{\mathbf{1 0} \times \mathbf{3}} \\
\mathbf{I}_{\mathbf{3} \times \mathbf{3}}
\end{array}\right] \underline{y}_{T, c}
$$

Or equivalently, written as

$$
\Delta \underline{\dot{x}}_{T_{A}}=\mathbf{A}_{\mathbf{T}_{\mathbf{C L}, \mathbf{i}}} \Delta \underline{x}_{T_{A}}-\left[\begin{array}{c}
\mathbf{0}_{10 \times \mathbf{3}} \\
\mathbf{I}_{\mathbf{3} \times \mathbf{3}}
\end{array}\right] \underline{y}_{T, c}
$$


where $\Delta \underline{x}_{T_{A}}=\left[\Delta \underline{x}_{T}^{T} \underline{e}_{T}^{T}\right]^{T}$ now represents the full augmented state vector and $\mathbf{A}_{\mathbf{T}_{\mathbf{C L}, \mathbf{i}}}$ represents the closed loop dynamics matrix of the tanker.

Employment of the linear control law in Eq. (3.8) into the full order nonlinear system requires realization that the feedback gain computes deviations of the control variables from their nominal values, based on state deviations, $\Delta \underline{x}_{T_{A}}$, multiplied by the Kalman gain. The actual state and control vectors are written as the sum of the nominal values and the perturbations from the nominal values as

$$
\begin{aligned}
& \underline{x}_{T_{A}}=\underline{x}_{T_{A_{o, i}}}+\Delta \underline{x}_{T_{A}} \\
& \underline{u}_{T, i}=\underline{u}_{T_{o, i}}+\Delta \underline{u}_{T, i}
\end{aligned}
$$

where $\underline{x}_{T_{A_{o, i}}}$ and $\underline{u}_{T_{o, i}}$ denote nominal state and control action values, respectively. In other words, the total control action is found by adding the computed control deviations to their nominal values. Solving for the deviations, the state perturbations are calculated in real time as

$$
\Delta \underline{x}_{T_{A}}=\underline{x}_{T_{A}}-\underline{x}_{T_{A_{o, i}}}
$$

and the control action perturbations are calculated as

$$
\Delta \underline{u}_{T, i}=\underline{u}_{T, i}-\underline{u}_{T_{o, i}}
$$

Substitution of Eq. (3.12) and Eq. (3.13) into the linear control law in Eq. (3.8) yields the final implementable form

$$
\underline{u}_{T, i}=\underline{u}_{T_{o, i}}-\mathbf{K}_{\mathbf{T}, \mathbf{i}}\left(\underline{x}_{T_{A}}-\underline{x}_{T_{A_{o, i}}}\right)
$$

to be scheduled between nominal conditions for real time execution. For this task, Eq. (3.14) is computed for the $i^{\text {th }}$ nominal condition and the scheduled control action is 
calculated by interpolating among all the nominal conditions using a Lagrange interpolation scheme mechanized as [1]

$$
\begin{aligned}
\underline{u}_{T}= & {\left[\frac{\left(\phi_{T, C}-\phi_{T, 2}\right)\left(\phi_{T, C}-\phi_{T, 3}\right)}{\left(\phi_{T, 1}-\phi_{T, 2}\right)\left(\phi_{T, 1}-\phi_{T, 3}\right)}\right]\left[\frac{\left(V_{T, C}-V_{T, 2}\right)}{\left(V_{T, 1}-V_{T, 2}\right)} \underline{u}_{T, 1}+\frac{\left(V_{T, C}-V_{T, 1}\right)}{\left(V_{T, 2}-V_{T, 1}\right)} \underline{u}_{T, 2}\right] } \\
& {\left[\frac{\left(\phi_{T, C}-\phi_{T, 1}\right)\left(\phi_{T, C}-\phi_{T, 3}\right)}{\left(\phi_{T, 2}-\phi_{T, 1}\right)\left(\phi_{T, 2}-\phi_{T, 3}\right)}\right]\left[\frac{\left(V_{T, C}-V_{T, 2}\right)}{\left(V_{T, 1}-V_{T, 2}\right)} \underline{u}_{T, 3}+\frac{\left(V_{T, C}-V_{T, 1}\right)}{\left(V_{T, 2}-V_{T, 1}\right)} \underline{u}_{T, 4}\right] } \\
& {\left[\frac{\left(\phi_{T, C}-\phi_{T, 1}\right)\left(\phi_{T, C}-\phi_{T, 2}\right)}{\left(\phi_{T, 3}-\phi_{T, 1}\right)\left(\phi_{T, 3}-\phi_{T, 2}\right)}\right]\left[\frac{\left(V_{T, C}-V_{T, 2}\right)}{\left(V_{T, 1}-V_{T, 2}\right)} \underline{u}_{T, 5}+\frac{\left(V_{T, C}-V_{T, 1}\right)}{\left(V_{T, 2}-V_{T, 1}\right)} \underline{u}_{T, 6}\right] }
\end{aligned}
$$

$V_{T, C}$ and $\phi_{T, C}$ are the total velocity and bank attitude commands, respectively, used as the scheduling independent variables. $\underline{u}_{T, i}$ denotes the total control action required at each specific nominal condition given by Eq. (3.14), and finally $V_{T, i}$ and $\phi_{T, i}$ are the nominal values of the scheduling variables at each nominal condition.

\subsection{Receiver}

The receiver vehicle open loop linearized state-space system including disturbance is described per nominal condition $i$ by the following:

$$
\Delta \underline{\dot{x}}=\mathbf{A}_{i} \Delta \underline{x}+\mathbf{B}_{i} \Delta \underline{u}+\mathbf{H}_{i}(\mathbf{t}) \Delta \underline{w}
$$

with state vector,

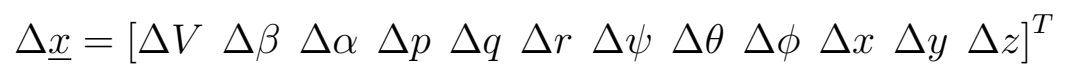

and control input vector,

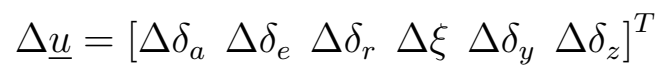

where index $i$ refers to the $i^{\text {th }}$ nominal condition and in addition to the conventional effectors (aileron, elevator, rudder, throttle), $\delta_{y}$ and $\delta_{z}$ define thrust vectoring control inputs. The disturbance vector due to the motion of the tanker is given by

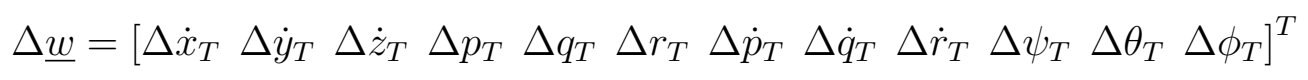


Here, a proposition is introduced that there exists a matrix $\Upsilon \mathbf{( t )} \in \Re^{12 x 13}$ which can be used as a linear transformation from the tanker state vector to the disturbance vector of the receiver as

$$
\Delta \underline{w}=\Upsilon(\mathbf{t}) \Delta \underline{x}_{T_{A}}
$$

Substituting this equation into Eq. (3.16) yields

$$
\Delta \underline{\dot{x}}=\mathbf{A}_{i} \Delta \underline{x}+\mathbf{B}_{i} \Delta \underline{u}+\mathbf{E}_{i} \Delta \underline{x}_{T_{A}}
$$

where

$$
\mathbf{E}_{i}=\mathbf{H}_{i}(\mathbf{t}) \Upsilon_{i}(\mathbf{t}) \in \Re^{12 x 13}
$$

Note that the product of these two time-varying matrices yields a constant matrix. This proposition will be proven in Chapter 4. Matrix $\mathbf{E}_{i}$ can be decomposed as

$$
\mathbf{E}_{i}=\left[\begin{array}{ll}
\mathbf{E}_{\mathbf{x}_{\mathbf{T}, \mathbf{i}}} & \mathbf{E}_{\mathbf{e}_{\mathbf{T}, \mathbf{i}}}
\end{array}\right]
$$

where $\mathbf{E}_{\mathbf{x}_{\mathbf{T}, \mathbf{i}}} \in \Re^{12 x 10}$ and $\mathbf{E}_{\mathbf{e}_{\mathbf{T}, \mathbf{i}}} \in \Re^{12 x 3}$.

The outer loop output vector for the receiver is chosen as relative position from the tanker vehicle,

$$
\underline{y}=\left[\begin{array}{lll}
\Delta x & \Delta y & \Delta z
\end{array}\right]^{T}
$$

and the error control variable is defined as the difference between the output vector and a desired relative position.

$$
\underline{\dot{e}}=\underline{y}-\underline{y_{c}}
$$

Expanding to scalar form for reference,

$$
\begin{aligned}
& \dot{e}_{x}=\Delta x-\Delta x_{c} \\
& \dot{e}_{y}=\Delta y-\Delta y_{c} \\
& \dot{e}_{z}=\Delta z-\Delta z_{c}
\end{aligned}
$$


Repeating the equivalent procedure utilized to augment the tanker open loop equation, the receiver error vector is adjoined with the receiver states to formulate an augmented open loop state-space equation as follows:

$$
\left[\begin{array}{c}
\Delta \underline{\dot{x}} \\
\underline{\dot{e}}
\end{array}\right]=\left[\begin{array}{cc}
\mathbf{A}_{i} & \mathbf{0}_{\mathbf{1 2} \times \mathbf{3}} \\
\mathbf{C}_{, \mathbf{i}} & \mathbf{0}_{\mathbf{3} \times \mathbf{3}}
\end{array}\right]\left[\begin{array}{c}
\Delta \underline{x} \\
\underline{e}
\end{array}\right]+\left[\begin{array}{c}
\mathbf{B}_{i} \\
\mathbf{0}_{\mathbf{3} \times \mathbf{6}}
\end{array}\right] \Delta \underline{u}+\left[\begin{array}{c}
\mathbf{E}_{i} \\
\mathbf{0}_{\mathbf{3} \times \mathbf{6}}
\end{array}\right] \Delta \underline{x}_{T_{A}}-\left[\begin{array}{c}
\mathbf{0}_{\mathbf{1 2} \times \mathbf{3}} \\
\mathbf{I}_{\mathbf{3} \times \mathbf{3}}
\end{array}\right] \underline{y_{c}}
$$

Next, as shown in Eq. (2.16) per the Friedland procedure to reduce a known exogenous quantity into the state-space equation [20], the receiver open loop equation is augmented once more with the tanker state vector $\underline{x}_{T}$ and the tanker error vector $\underline{e}_{T}$ to formulate the two vehicle metastate-space open loop system as follows:

$$
\begin{aligned}
& {\left[\begin{array}{c}
\Delta \underline{\dot{x}} \\
\underline{\dot{e}} \\
\Delta \underline{\dot{x}}_{T} \\
\underline{e}_{T}
\end{array}\right]=\left[\begin{array}{llll}
\mathbf{A}_{i} & \mathbf{0}_{\mathbf{1 2 \times 3}} & \mathbf{E}_{\mathbf{x}_{\mathbf{T}, \mathbf{i}}} & \mathbf{E}_{\mathbf{e}_{\mathbf{T}, \mathbf{i}}} \\
\mathbf{C}_{, \mathbf{i}} & \mathbf{0}_{\mathbf{3} \times \mathbf{3}} & \mathbf{0}_{\mathbf{3} \times \mathbf{1 0}} & \mathbf{0}_{\mathbf{3} \times \mathbf{3}} \\
\mathbf{0}_{10 \times \mathbf{1 2}} & \mathbf{0}_{\mathbf{1 0} \times \mathbf{3}} & \left(\mathbf{A}_{T, i}-\mathbf{B}_{T, i} \mathbf{K}_{\mathbf{x}_{\mathbf{T}, \mathbf{i}}}\right) & -\mathbf{B}_{T, i} \mathbf{K}_{\mathbf{e}_{\mathbf{T}, \mathbf{i}}} \\
\mathbf{0}_{\mathbf{3} \times \mathbf{1 2}} & \mathbf{0}_{\mathbf{3} \times \mathbf{3}} & \mathbf{C}_{\mathbf{T}, \mathbf{i}} & \mathbf{0}_{\mathbf{3} \times \mathbf{3}}
\end{array}\right]\left[\begin{array}{c}
\Delta \underline{x} \\
\underline{e} \\
\Delta \underline{x}_{T} \\
\underline{e}_{T}
\end{array}\right]} \\
& +\left[\begin{array}{c}
\mathbf{B}_{i} \\
\mathbf{0}_{3 \times 6} \\
\mathbf{0}_{10 \times 6} \\
\mathbf{0}_{3 \times 6}
\end{array}\right] \Delta \underline{u}-\left[\begin{array}{c}
\mathbf{0}_{12 \times \mathbf{3}} \\
\mathbf{I}_{3 \times \mathbf{3}} \\
\mathbf{0}_{10 \times \mathbf{3}} \\
\mathbf{0}_{\mathbf{3} \times \mathbf{3}}
\end{array}\right] \underline{y_{c}}-\left[\begin{array}{c}
\mathbf{0}_{12 \times \mathbf{3}} \\
\mathbf{0}_{\mathbf{3} \times \mathbf{3}} \\
\mathbf{0}_{10 \times \mathbf{3}} \\
\mathbf{I}_{3 \times \mathbf{3}}
\end{array}\right] \underline{y}_{T, c}
\end{aligned}
$$

Finally, the metastate matrices $\mathbf{A}_{\mathbf{o}}$ and $\mathbf{B}_{\mathbf{o}}$ (presented in expanded form in Eq. (3.28)) are used in the LQR procedure, referenced in Chapter 2, to compute the feedback gain matrix utilized in the linear control law of the form

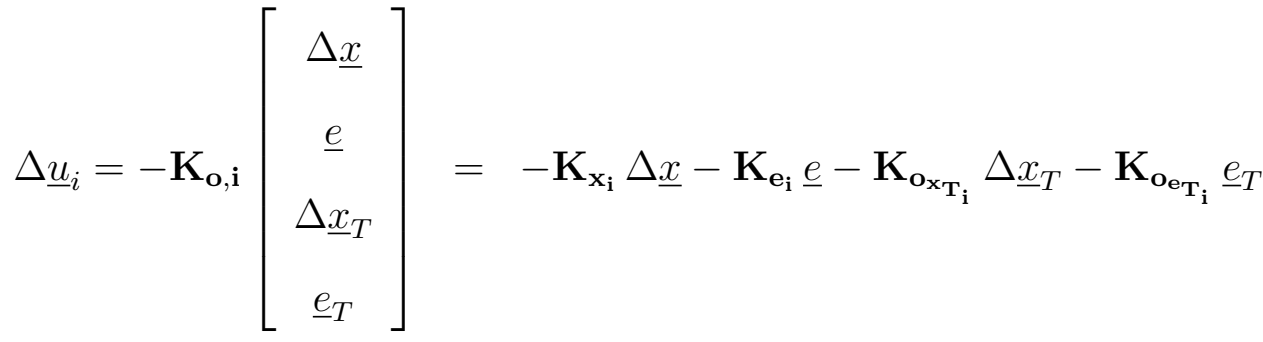

$$
\begin{aligned}
& =-\mathbf{K}_{\mathbf{x}_{\mathbf{i}}} \Delta \underline{x}-\mathbf{K}_{\mathbf{e}_{\mathbf{i}} \underline{e}}-\mathbf{K}_{\mathbf{o}_{\mathbf{T}_{\mathbf{i}}}} \Delta \underline{x}_{T_{A}}
\end{aligned}
$$


The receiver linear control law in Eq. (3.29) is employed into the full order nonlinear model in an equivalent manner as the tanker controller. Defining the complete state

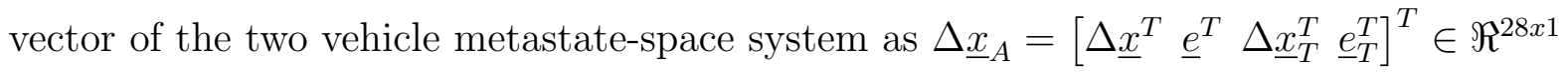
and rewriting the receiver linear control in Eq. (3.29) as

$$
\Delta \underline{u}=-\mathbf{K}_{\mathbf{o}, \mathbf{i}} \underline{x}_{A}
$$

the total control action is found by considering deviations for both the state $\underline{x}_{A}$ and receiver control variables, $\underline{u}$, about each nominal condition, $i$. The actual vectors are written as the sum of the nominal values plus a perturbation as

$$
\begin{aligned}
& \underline{x}_{A}=\underline{x}_{A_{o, i}}+\Delta \underline{x}_{A} \\
& \underline{u}_{, i}=\underline{u}_{o, i}+\Delta \underline{u}_{, i}
\end{aligned}
$$

where $\underline{x}_{A_{o, i}}$ and $\underline{u}_{o, i}$ denote receiver nominal state and control action values, respectively. Solving for perturbations,

$$
\begin{aligned}
\Delta \underline{x}_{A} & =\underline{x}_{A}-\underline{x}_{A_{o, i}} \\
\Delta \underline{u}_{, i} & =\underline{u}_{, i}-\underline{u}_{o, i}
\end{aligned}
$$

Substitution of Eq. (3.32) into the linear control law in Eq. (3.30) yields the form suitable for a gain schedule in real time execution.

$$
\underline{u}_{, i}=\underline{u}_{o, i}-\mathbf{K}_{\mathbf{o}, \mathbf{i}}\left(\underline{x}_{A}-\underline{x}_{A_{o, i}}\right)
$$

Reiterating an equivalent procedure as used in the tanker controller, the gain scheduling task for the receiver computes $\underline{u}_{, i}$ in Eq. (3.33) for the $i^{\text {th }}$ nominal condition in real time 
with the state vector, $\underline{x}_{A}$. Next, the total receiver control action required, $\underline{u}$, is computed using the following Lagrange interpolation scheme: [1]

$$
\begin{aligned}
\underline{u}= & {\left[\frac{\left(\phi_{T, C}-\phi_{T, 2}\right)\left(\phi_{T, C}-\phi_{T, 3}\right)}{\left(\phi_{T, 1}-\phi_{T, 2}\right)\left(\phi_{T, 1}-\phi_{T, 3}\right)}\right]\left[\frac{\left(V_{T, C}-V_{T, 2}\right)}{\left(V_{T, 1}-V_{T, 2}\right)} \underline{u}_{, 1}+\frac{\left(V_{T, C}-V_{T, 1}\right)}{\left(V_{T, 2}-V_{T, 1}\right)} \underline{u}_{, 2}\right] } \\
& {\left[\frac{\left(\phi_{T, C}-\phi_{T, 1}\right)\left(\phi_{T, C}-\phi_{T, 3}\right)}{\left(\phi_{T, 2}-\phi_{T, 1}\right)\left(\phi_{T, 2}-\phi_{T, 3}\right)}\right]\left[\frac{\left(V_{T, C}-V_{T, 2}\right)}{\left(V_{T, 1}-V_{T, 2}\right)} \underline{u}_{, 3}+\frac{\left(V_{T, C}-V_{T, 1}\right)}{\left(V_{T, 2}-V_{T, 1}\right)} \underline{u}_{, 4}\right] } \\
& {\left[\frac{\left(\phi_{T, C}-\phi_{T, 1}\right)\left(\phi_{T, C}-\phi_{T, 2}\right)}{\left(\phi_{T, 3}-\phi_{T, 1}\right)\left(\phi_{T, 3}-\phi_{T, 2}\right)}\right]\left[\frac{\left(V_{T, C}-V_{T, 2}\right)}{\left(V_{T, 1}-V_{T, 2}\right)} \underline{u}_{, 5}+\frac{\left(V_{T, C}-V_{T, 1}\right)}{\left(V_{T, 2}-V_{T, 1}\right)} \underline{u}_{, 6}\right] }
\end{aligned}
$$

The coefficients of $V_{T, C}, \phi_{T, C}, V_{T, i}$, and $\phi_{T, i}$ are the equivalent total velocity and bank attitude commands and nominal values of these scheduling variables as employed in the tanker gain scheduler. The sole difference for the receiver Lagrange interpolation scheme is the $\underline{u}_{, i}$ total control action required for the receiver at each specific nominal condition, given by Eq. (3.33). 


\section{CHAPTER 4}

\section{TRANSFORMATION FROM TANKER STATE VECTOR TO RECEIVER DISTURBANCE VECTOR}

Implementation of the disturbance rejection approach [20] in the receiver stationkeeping control law requires transformation to the disturbance vector in the receiver linearized equation of motion in Eq. (2.12) from the augmented state vector for the tanker as defined in Eq. (3.7). Recalling Eq. (3.2) and adjoining error states, the augmented tanker state vector is

$$
\underline{x}_{T_{A}}=\left[\begin{array}{lllllllllllll}
V_{T} & \beta_{T} & \alpha_{T} & p_{T} & q_{T} & r_{T} & \theta_{T} & \phi_{T} & z_{T} & \psi_{T} & e_{V_{T}} & e_{z_{T}} & e_{\phi_{T}}
\end{array}\right]^{T} \in \Re^{13 x 1}
$$

where the final three components represent error elements for the tanker autopilot type one control action (i.e. total velocity, altitude, and bank attitude integral errors where the error is defined as the respective state minus the reference command). Comparing the existing tanker state vector, $\underline{x}_{T_{A}}$ in Eq. (4.1) to the receiver disturbance vector, $\underline{w}$ (reference recalled Eq. (2.13) below), a direct substitution of the tanker states into the exogenous vector for the disturbance rejection approach is not readily possible.

$$
\underline{w}=\left[\begin{array}{llllllllllll}
\dot{x}_{T} & \dot{y}_{T} & \dot{z}_{T} & p_{T} & q_{T} & r_{T} & \dot{p}_{T} & \dot{q}_{T} & \dot{r}_{T} & \psi_{T} & \theta_{T} & \phi_{T}
\end{array}\right]^{T} \in \Re^{12 x 1}
$$

In order to utilize the existing tanker state vector as the exogenous term (note the expressions of exogenous and disturbance vector are used interchangeably) in the receiver state-space, an inspection is performed on the disturbance vector and the tanker state vector on an element by element basis. Comparing the first three elements in the state vector to the disturbance vector, observe that both arrays describe the equivalent tanker translational kinematics in the absence of wind, although the former is expressed in the wind frame as opposed to the inertial frame. Therefore, a relation to map velocities from 
one representation to the other is identified by resorting to the matrix form of the tanker translational kinematics relative to the inertial frame (and represented in the inertial frame), given by

$$
\dot{r}_{B_{T}}=\mathbf{R}_{\mathbf{B}_{\mathbf{T}} \mathbf{I}}^{\mathbf{T}} \mathbf{R}_{\mathbf{B}_{\mathbf{T}} \mathbf{w}_{\mathbf{T}}} V_{w_{T}}
$$

Note, Eq. (4.2) is obtained from the Eq. (2.3) in the absence of winds (i.e. $W=0$ ). (This approximation is consistent throughout the formulation of the linear model and controller design as an established step in prior research. The inclusion of an approximation for winds in these steps is a recommended investigation, discussed further in Chapter 6).

Expanding the nonlinear scalar equations, the transformation mapping for the first three elements in the inertial-represented state vector to the wind-represented disturbance begins to emerge clearly (reference Eqs. (4.3) - (4.5)).

$$
\begin{aligned}
\dot{x}_{T}= & V_{T}\left[\cos \beta_{T} \cos \alpha_{T} \cos \theta_{T} \cos \psi_{T}+\sin \beta_{T}\left(-\cos \phi_{T} \sin \psi_{T}+\sin \phi_{T} \sin \theta_{T} \cos \psi_{T}\right)\right. \\
& \left.+\cos \beta_{T} \sin \alpha_{T}\left(\sin \phi_{T} \sin \psi_{T}+\cos \phi_{T} \sin \theta_{T} \cos \psi_{T}\right)\right] \\
\dot{y}_{T}=V_{T}\left[\cos \beta_{T} \cos \alpha_{T} \cos \theta_{T} \sin \psi_{T}+\sin \beta_{T}\left(\cos \phi_{T} \cos \psi_{T}+\sin \phi_{T} \sin \theta_{T} \sin \psi_{T}\right)\right. & \\
& \left.\quad+\cos \beta_{T} \sin \alpha_{T}\left(-\sin \phi_{T} \cos \psi_{T}+\cos \phi_{T} \sin \theta_{T} \sin \psi_{T}\right)\right] \\
\dot{z}_{T}=\quad & V_{T}\left[-\cos \beta_{T} \cos \alpha_{T} \sin \theta_{T}+\sin \beta_{T} \sin \phi_{T} \cos \theta_{T}\right. \\
& \left.\quad+\cos \beta_{T} \sin \alpha_{T} \cos \phi_{T} \cos \theta_{T}\right]
\end{aligned}
$$

Finally, linearizing the translational kinematics equations about a given tanker nominal condition yields a transformation mapping in a suitable form for implementation into the LQR MIMO $[1,9,10]$ position tracking controller,

$$
\begin{aligned}
\Delta \dot{x}_{T} & =\mathrm{X}_{V_{T_{o}}} \Delta V_{T}+\mathrm{X}_{\beta_{T_{o}}} \Delta \beta_{T}+\mathrm{X}_{\alpha_{T_{o}}} \Delta \alpha_{T}+\mathrm{X}_{\theta_{T_{o}}} \Delta \theta_{T}+\mathrm{X}_{\phi_{T_{o}}} \Delta \phi_{T}+\mathrm{X}_{\psi_{T_{o}}} \Delta \psi_{T}\left(4.6 \mathrm{Y}_{\beta_{T_{o}}} \Delta \beta_{T}+\mathrm{Y}_{\alpha_{T_{o}}} \Delta \alpha_{T}+\mathrm{Y}_{\theta_{T_{o}}} \Delta \theta_{T}+\mathrm{Y}_{\phi_{T_{o}}} \Delta \phi_{T}+\mathrm{Y}_{\psi_{T_{o}}} \Delta \psi_{T}(4.7\right. \\
\Delta \dot{y}_{T} & =\mathrm{Y}_{V_{T_{o}}} \Delta V_{T}+\mathrm{Y}_{\beta_{T_{o}}} \Delta \beta_{T}+\mathrm{Z}_{\alpha_{T_{o}}} \Delta \alpha_{T}+\mathrm{Z}_{\theta_{T_{o}}} \Delta \theta_{T}+\mathrm{Z}_{\phi_{T_{o}}} \Delta \phi_{T} \\
\Delta \dot{z}_{T} & =\mathrm{Z}_{V_{T_{o}}} \Delta V_{T}+\mathrm{Z}_{\beta_{T}}
\end{aligned}
$$


where $\Delta$ represents deviation from the nominal condition, and new coefficient notations on the right hand side of the equations represent partial derivative components with respect to each tanker state (again evaluated at the nominal condition). For example, the first element in Eq. (4.6) denotes

$$
\left.\mathrm{X}_{V_{T_{o}}} \equiv \frac{\partial \dot{x}_{T}}{\partial V_{T}}\right|_{o}
$$

and similarly for the remaining coefficients as well as the terms in Eq. (4.7) and Eq. (4.8). Also of note at this point in the approach is the time variance introduced in the mapping due to dependency on tanker heading, $\psi_{T}$, in the coefficient terms within the $\dot{x}_{T}$ and $\dot{y}_{T}$ linearized translational equations, which presents a hurdle as the LQR MIMO $[1,9,10]$ position tracking controller requires a time invariant state-space system. Ideally, the most convenient implementation of the Friedland disturbance rejection methodology [20] in the application will preserve time invariance in the receiver control law, allowing an equivalent architecture to remain in place. Unfortunately, accounting for the receiver disturbance [20] induced by a tanker heading deviation in this particular case with the tanker translational kinematics appears to offer no convenient path forward. Optimism is maintained however with the benefit of hindsight. In short, a simple re-facilitation of the current time invariant control law is not viable with this new disturbance due to operating conditions where the tanker is in a steady-level turn, which constitutes a changing $\psi_{T}$ as a function of time, i.e., if in a steady turn, $\dot{\psi}_{T}$ is presumably a constant, $C$, then

$$
\psi_{T_{o}}=\int \dot{\psi}_{T_{o}} d \tau=\int C d \tau=C t+C_{1}=f(t)
$$

Therefore, the mapping transformation defining the first three elements from tanker state to receiver disturbance array is a time varying function describing inertial $x_{T}$ and $y_{T}$ position due to these $\psi_{T_{o}}$ components. Note, $z_{T}$ position is not a function of tanker heading as one would expect and yields a time invariant map (i.e. altitude is not a 
function of heading). In summary, with the given findings at this point regarding the translational kinematics mapping from wind frame (tanker state $\underline{x}_{T_{A}}$ ) to inertial frame (receiver disturbance $\underline{w}$ ), the worst case foreseen is that the receiver control architecture will require revision to execute as a time varying controller.

Moving forward with the definition of the mapping transformation, the next three elements (four through six) in the receiver disturbance vector $\underline{w}$ are the tanker body axis angular velocity components $\left(p_{T}, q_{T}\right.$, and $\left.r_{T}\right)$, and are all common to the tanker state vector, $\underline{x}_{T_{A}}$. Therefore, the mapping in this case is simply unity. No transformation is required to be defined here assuming the tanker states are available to the receiver vehicle.

Elements seven through nine in the receiver disturbance vector define the tanker body axis angular acceleration components $\left(\dot{p}_{T}, \dot{q}_{T}\right.$, and $\left.\dot{r}_{T}\right)$ and are not immediately available in the existing tanker state vector. However, as aforementioned in elements four through six, the integrals of these components are tanker states as the angular velocities. Given that the LQR MIMO $[1,9,10]$ position tracking controller gains are computed offline (i.e. before real time operation), the closed loop matrix $\mathbf{A}_{\mathbf{T}_{\mathbf{C L}}}$ directly provides the transformation required. The closed loop state-space equation offers a direct mapping from tanker state to receiver disturbance (in this case, components of $\underline{\dot{x}}_{T_{A}}$ ). Therefore, rows four through six are extracted out of the closed loop equation below

$$
\underline{\dot{x}}_{T_{A}}=\mathbf{A}_{\mathbf{T}_{\mathrm{CL}}} \underline{x}_{T_{A}}
$$


to define the transformation from tanker states to the tanker angular acceleration for the receiver disturbance. In scalar form,

$$
\begin{aligned}
& \dot{p}_{T}=A_{T_{C L_{4,1}}} V_{T}+A_{T_{C L_{4,2}}} \beta_{T}+A_{T_{C L_{4,3}}} \alpha_{T}+A_{T_{C L_{4,4}}} p_{T}+A_{T_{C L_{4}, 5}} q_{T}+A_{T_{C L_{4,6}}} r_{T} \\
& +A_{T_{C L_{4}, 7}} \theta_{T}+A_{T_{C L_{4,8}}} \phi_{T}+A_{T_{C L_{4}, 9}} z_{T}+A_{T_{C L_{4}, 10}} \psi_{T}+A_{T_{C L_{4}, 11}} e_{V_{T}}+A_{T_{C L_{4}, 12}} e_{z_{T}} \\
& +A_{T_{C L_{4,13}}} e_{\phi_{T}} \\
& \dot{q}_{T}=A_{T_{C L_{5,1}}} V_{T}+A_{T_{C L_{5}, 2}} \beta_{T}+A_{T_{C L_{5}, 3}} \alpha_{T}+A_{T_{C L_{5}, 4}} p_{T}+A_{T_{C L_{5}, 5}} q_{T}+A_{T_{C L_{5}, 6}} r_{T} \\
& +A_{T_{C L_{5}, 7}} \theta_{T}+A_{T_{C L_{5}, 8}} \phi_{T}+A_{T_{C L_{5}, 9}} z_{T}+A_{T_{C L_{5}, 10}} \psi_{T}+A_{T_{C L_{5}, 11}} e_{V_{T}}+A_{T_{C L_{5}, 12}} e_{z_{T}} \\
& +A_{T_{C L 5,13}} e_{\phi_{T}} \\
& \dot{r}_{T}=A_{T_{C L_{6,1}}} V_{T}+A_{T_{C L_{6,2}}} \beta_{T}+A_{T_{C L_{6,3}}} \alpha_{T}+A_{T_{C L_{6,4}}} p_{T}+A_{T_{C L_{6}, 5}} q_{T}+A_{T_{C L_{6,6}}} r_{T} \\
& +A_{T_{C L_{6}, 7}} \theta_{T}+A_{T_{C L_{6,8}}} \phi_{T}+A_{T_{C L_{6,9}}} z_{T}+A_{T_{C L_{6,10}}} \psi_{T}+A_{T_{C L_{6,11}}} e_{V_{T}}+A_{T_{C L_{6,12}}} e_{z_{T}} \\
& +A_{T_{C L_{6,13}}} e_{\phi_{T}}
\end{aligned}
$$

where $A_{T_{C L_{i, j}}}$ denotes the $i^{\text {th }}$ row and $j^{\text {th }}$ column element from the $\mathbf{A}_{\mathbf{T}_{\mathbf{C L}}}$ tanker closed loop matrix in Eq. (4.10).

Finally, the remaining three components in the receiver disturbance array $\left(\psi_{T}, \theta_{T}\right.$, and $\phi_{T}$ ) are also already common to the tanker state vector, $\underline{x}_{T_{A}}$. Therefore again, as with elements four through six, the mapping is unity and allows direct implementation into the receiver disturbance vector, $\underline{w}$.

Combining Eqs. (4.6) - (4.8) and Eqs. (4.11) - (4.13), compatibility between the receiver state-space equation and the tanker state-space equation as the disturbance model is established with the transformation matrix $\Upsilon$ to map augmented tanker state vector, $\underline{x}_{T_{A}} \in \Re^{13 x 1}$ to the receiver disturbance vector $\underline{w} \in \Re^{12 x 1}$. Recalling Eq. (3.20),

$$
\Delta \underline{w}=\Upsilon(\mathbf{t}) \Delta \underline{x}_{T_{A}}
$$

where transformation matrix $\Upsilon \mathbf{( t )} \in \Re^{12 \times 13}$ contains the resultant mappings discussed in detail above. Note in the interest of presenting $\mathbf{\Upsilon}(\mathbf{t})$ in the limited space below, 
the parenthetical dual numbered elements indicate the corresponding row and column component of the tanker closed loop state matrix. For example, $(4,1)$ denotes the fourth row, first column component of $\mathbf{A}_{\mathbf{T}_{\mathrm{CL}}}$ as observed in Eq. (4.11) for the $V_{T}$ coefficient.

$$
\Upsilon_{(\mathbf{t})}=\left[\begin{array}{ccccccccccccc}
\mathrm{X}_{V_{T_{O}}}(t) & \mathrm{X}_{\beta_{T_{O}}}(t) & \mathrm{X}_{\alpha_{T_{O}}}(t) & 0 & 0 & 0 & \mathrm{X}_{\theta_{T_{O}}}(t) & \mathrm{X}_{\phi_{T_{o}}}(t) & 0 & \mathrm{X}_{\psi_{T_{O}}}(t) & 0 & 0 & 0 \\
\mathrm{Y}_{V_{T_{O}}}(t) & \mathrm{Y}_{\beta_{T_{O}}}(t) & \mathrm{Y}_{\alpha_{T_{O}}}(t) & 0 & 0 & 0 & \mathrm{Y}_{\theta_{T_{O}}}(t) & \mathrm{Y}_{\phi_{T_{O}}}(t) & 0 & \mathrm{Y}_{\psi_{T_{O}}}(t) & 0 & 0 & 0 \\
\mathrm{Z}_{V_{T_{O}}} & \mathrm{Z}_{\beta_{T_{O}}} & \mathrm{Z}_{\alpha_{T_{O}}} & 0 & 0 & 0 & \mathrm{Z}_{\theta_{T_{O}}} & \mathrm{Z}_{\phi_{T_{O}}} & 0 & 0 & 0 & 0 & 0 \\
0 & 0 & 0 & 1 & 0 & 0 & 0 & 0 & 0 & 0 & 0 & 0 & 0 \\
0 & 0 & 0 & 0 & 1 & 0 & 0 & 0 & 0 & 0 & 0 & 0 & 0 \\
0 & 0 & 0 & 0 & 0 & 1 & 0 & 0 & 0 & 0 & 0 & 0 & 0 \\
(4,1) & (4,2) & (4,3) & (4,4) & (4,5) & (4,6) & (4,7) & (4,8) & (4,9) & (4,10) & (4,11) & (4,12) & (4,13) \\
(5,1) & (5,2) & (5,3) & (5,4) & (5,5) & (5,6) & (5,7) & (5,8) & (5,9) & (5,10) & (5,11) & (5,12) & (5,13) \\
(6,1) & (6,2) & (6,3) & (6,4) & (6,5) & (6,6) & (6,7) & (6,8) & (6,9) & (6,10) & (6,11) & (6,12) & (6,13) \\
0 & 0 & 0 & 0 & 0 & 0 & 0 & 0 & 0 & 1 & 0 & 0 & 0 \\
0 & 0 & 0 & 0 & 0 & 0 & 1 & 0 & 0 & 0 & 0 & 0 & 0 \\
0 & 0 & 0 & 0 & 0 & 0 & 0 & 1 & 0 & 0 & 0 & 0 & 0
\end{array}\right]
$$

In summary, unity elements in $\Upsilon$ indicate a direct mapping from the modified tanker state vector $\underline{x}_{T_{A}}$ to receiver disturbance $\underline{w}$. The first three rows of the transformation matrix constitute the linearized tanker translational kinematics transformed from wind frame $\left[\begin{array}{lll}V_{T} & \beta_{T} & \alpha_{T}\end{array}\right]^{T}$ to inertial frame $\left[\begin{array}{lll}\dot{x}_{T} & \dot{y}_{T} & \dot{z}_{T}\end{array}\right]^{T}$ where individual elements represent the partial derivative coefficients evaluated at the nominal condition as shown in Eqs. (4.6) - (4.8). Note coefficients include time variant components due to inertial heading $\psi_{T_{o}}(t)$ dependency as previously discussed. And finally, rows seven through nine are plumbed directly from the closed loop dynamics equation governing the tanker angular acceleration.

As discussed in Chapter 3, substitution of Eq. (4.14) into Eq. (2.12) yields

$$
\underline{\dot{x}}=\mathbf{A} \underline{x}+\mathbf{B} \underline{u}+\mathbf{H}(\mathbf{t}) \mathbf{\Upsilon}(\mathbf{t}) \underline{x}_{T_{A}}
$$

delivering the necessary form to apply the Friedland disturbance rejection approach with the existing definition of the tanker state vector and known closed loop dynamics equation (Eq. (4.10)). However, the current control methodology calls for a time invariant system, and despite augmentation into a open loop metasystem (Eq. (2.16)), $\mathbf{A}_{\mathbf{o}}$ will remain a function of time due to $\mathbf{H}(\mathbf{t})$ and $\boldsymbol{\Upsilon}(\mathbf{t})$. 
Previously, time variance was investigated in the newly defined transformation $\Upsilon$. Repeating this investigation for $\mathbf{H}(\mathbf{t})$ at the nominal condition of a steady-level tanker turn, time variance is based on two terms: (i) the inertial heading $\psi_{T_{o}}(t)$, and (ii) the inertial groundtrack velocities $\dot{x}_{T_{o}}(t)$, and $\dot{y}_{T_{o}}(t)$ which are implicitly time variant due to item (i) dependency. Inspection reveals that the tanker inertial heading and inertial groundtrack velocities are components described in $\mathbf{R}_{\mathbf{B}_{\mathbf{T}} \mathbf{I}}$ and $\dot{r}_{B_{T}}$, respectively, and appear only as exogenous terms governing motion between the two relative bodies in the receiver translational kinematics (Eq. (2.1)) and in the receiver translational dynamics (Eq. (2.4)). In the latter, only the rotational matrix $\mathbf{R}_{\mathbf{B}_{\mathbf{T}} \mathbf{I}}$ appears as a gravitational term $\left(\mathbf{R}_{\mathbf{B}_{\mathbf{T}} \mathbf{I}} M_{R}\right)$. Upon expansion of the receiver translational dynamics scalar equations, there is no dependency on the tanker heading, $\psi_{T}$. This result is intuitive as the receiver's acceleration due to gravity is not a function of whether the tanker vehicle is flying due North or South. Therefore, the time variance within $\mathbf{H}(\mathbf{t})$ is posed by the receiver translational kinematics equations alone. Reference $\mathbf{H}(\mathbf{t})$ below noting the appearance of time variant terms denoted with $(t)$.

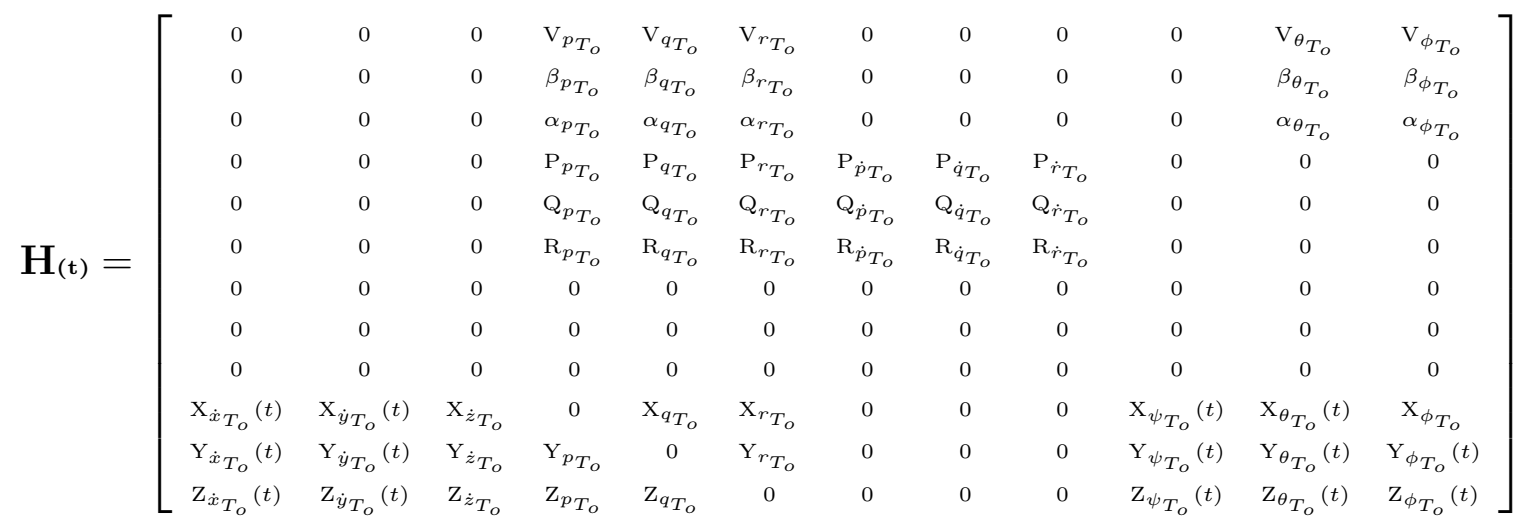

Additionally, the element notation above is abbreviated to preserve space, and again, coefficients denote partial derivatives of receiver states with respect to the disturbance vector, $\underline{w}$. For example, the element in row ten, column one in $\mathbf{H}(\mathbf{t})$ represents the time variant coefficient of the partial derivative of receiver relative $\dot{x}$ velocity with respect to tanker inertial velocity $\dot{x}_{T}$, evaluated at the nominal condition, as 


$$
\left.\mathrm{X}_{\dot{x}_{T_{o}}}(t) \equiv \frac{\partial \dot{x}}{\partial \dot{x}_{T}}\right|_{o}
$$

Computing the product $\mathbf{H}(\mathbf{t}) \mathbf{\Upsilon}(\mathbf{t})$ reveals the time variant components of $\mathbf{\Upsilon}(\mathbf{t})$ are multiplied by either i) zero elements or ii) additional time variant components in $\mathbf{H}(\mathbf{t})$. For clarity, the product $\mathbf{H}(\mathbf{t}) \Upsilon(\mathbf{t})$ is presented below with a substitution of $\mathrm{C}$ and $\mathrm{f}(t)$ for constant time invariant terms and time variant terms, respectively.

$$
\left[\begin{array}{cccccccccccc}
0 & 0 & 0 & \mathrm{C} & \mathrm{C} & \mathrm{C} & 0 & 0 & 0 & 0 & \mathrm{C} & \mathrm{C} \\
0 & 0 & 0 & \mathrm{C} & \mathrm{C} & \mathrm{C} & 0 & 0 & 0 & 0 & \mathrm{C} & \mathrm{C} \\
0 & 0 & 0 & \mathrm{C} & \mathrm{C} & \mathrm{C} & 0 & 0 & 0 & 0 & \mathrm{C} & \mathrm{C} \\
0 & 0 & 0 & \mathrm{C} & \mathrm{C} & \mathrm{C} & \mathrm{C} & \mathrm{C} & \mathrm{C} & 0 & 0 & 0 \\
0 & 0 & 0 & \mathrm{C} & \mathrm{C} & \mathrm{C} & \mathrm{C} & \mathrm{C} & \mathrm{C} & 0 & 0 & 0 \\
0 & 0 & 0 & \mathrm{C} & \mathrm{C} & \mathrm{C} & \mathrm{C} & \mathrm{C} & \mathrm{C} & 0 & 0 & 0 \\
0 & 0 & 0 & 0 & 0 & 0 & 0 & 0 & 0 & 0 & 0 & 0 \\
0 & 0 & 0 & 0 & 0 & 0 & 0 & 0 & 0 & 0 & 0 & 0 \\
0 & 0 & 0 & 0 & 0 & 0 & 0 & 0 & 0 & 0 & 0 & 0 \\
\mathrm{f}(t) & \mathrm{f}(t) & \mathrm{C} & 0 & \mathrm{C} & \mathrm{C} & 0 & 0 & 0 & \mathrm{f}(t) & \mathrm{f}(t) & \mathrm{C} \\
\mathrm{f}(t) & \mathrm{f}(t) & \mathrm{C} & \mathrm{C} & 0 & \mathrm{C} & 0 & 0 & 0 & \mathrm{f}(t) & \mathrm{f}(t) & \mathrm{f}(t) \\
\mathrm{f}(t) & \mathrm{f}(t) & \mathrm{C} & \mathrm{C} & \mathrm{C} & 0 & 0 & 0 & 0 & \mathrm{f}(t) & \mathrm{f}(t) & \mathrm{f}(t)
\end{array}\right]\left[\begin{array}{cccccccccccccc}
\mathrm{f}(t) & \mathrm{f}(t) & \mathrm{f}(t) & 0 & 0 & 0 & \mathrm{f}(t) & \mathrm{f}(t) & 0 & \mathrm{f}(t) & 0 & 0 & 0 \\
\mathrm{f}(t) & \mathrm{f}(t) & \mathrm{f}(t) & 0 & 0 & 0 & \mathrm{f}(t) & \mathrm{f}(t) & 0 & \mathrm{f}(t) & 0 & 0 & 0 \\
\mathrm{C} & \mathrm{C} & \mathrm{C} & 0 & 0 & 0 & \mathrm{C} & \mathrm{C} & 0 & 0 & 0 & 0 & 0 \\
0 & 0 & 0 & 1 & 0 & 0 & 0 & 0 & 0 & 0 & 0 & 0 & 0 \\
0 & 0 & 0 & 0 & 1 & 0 & 0 & 0 & 0 & 0 & 0 & 0 & 0 \\
0 & 0 & 0 & 0 & 0 & 1 & 0 & 0 & 0 & 0 & 0 & 0 & 0 \\
\mathrm{C} & \mathrm{C} & \mathrm{C} & \mathrm{C} & \mathrm{C} & \mathrm{C} & \mathrm{C} & \mathrm{C} & \mathrm{C} & \mathrm{C} & \mathrm{C} & \mathrm{C} & \mathrm{C} \\
\mathrm{C} & \mathrm{C} & \mathrm{C} & \mathrm{C} & \mathrm{C} & \mathrm{C} & \mathrm{C} & \mathrm{C} & \mathrm{C} & \mathrm{C} & \mathrm{C} & \mathrm{C} & \mathrm{C} \\
\mathrm{C} & \mathrm{C} & \mathrm{C} & \mathrm{C} & \mathrm{C} & \mathrm{C} & \mathrm{C} & \mathrm{C} & \mathrm{C} & \mathrm{C} & \mathrm{C} & \mathrm{C} & \mathrm{C} \\
0 & 0 & 0 & 0 & 0 & 0 & 0 & 0 & 0 & 1 & 0 & 0 & 0 \\
0 & 0 & 0 & 0 & 0 & 0 & 1 & 0 & 0 & 0 & 0 & 0 & 0 \\
0 & 0 & 0 & 0 & 0 & 0 & 0 & 1 & 0 & 0 & 0 & 0 & 0
\end{array}\right]
$$

In the case of (i), the resultant elements in the product, $\mathbf{E}$ as previously defined in Eq. (3.22), are zero and there is no time dependency. Eq. (4.16) depicts the location of these zero components due to the product operation. Again for brevity in display, $C$ denotes constant time invariant terms, and $f(t$ ? ) now represents potential time variant components resulting from the multiplication described in case (ii) above. The nature of these time variant terms in question will be addressed ahead.

$$
\mathbf{H}_{(\mathrm{t})} \boldsymbol{\Upsilon}_{(\mathrm{t})}=\mathbf{E}=\left[\begin{array}{ccccccccccccc}
0 & 0 & 0 & \mathrm{C} & \mathrm{C} & \mathrm{C} & \mathrm{C} & \mathrm{C} & 0 & 0 & 0 & 0 & 0 \\
0 & 0 & 0 & \mathrm{C} & \mathrm{C} & \mathrm{C} & \mathrm{C} & \mathrm{C} & 0 & 0 & 0 & 0 & 0 \\
0 & 0 & 0 & \mathrm{C} & \mathrm{C} & \mathrm{C} & \mathrm{C} & \mathrm{C} & 0 & 0 & 0 & 0 & 0 \\
\mathrm{C} & \mathrm{C} & \mathrm{C} & \mathrm{C} & \mathrm{C} & \mathrm{C} & \mathrm{C} & \mathrm{C} & \mathrm{C} & \mathrm{C} & \mathrm{C} & \mathrm{C} & \mathrm{C} \\
\mathrm{C} & \mathrm{C} & \mathrm{C} & \mathrm{C} & \mathrm{C} & \mathrm{C} & \mathrm{C} & \mathrm{C} & \mathrm{C} & \mathrm{C} & \mathrm{C} & \mathrm{C} & \mathrm{C} \\
\mathrm{C} & \mathrm{C} & \mathrm{C} & \mathrm{C} & \mathrm{C} & \mathrm{C} & \mathrm{C} & \mathrm{C} & \mathrm{C} & \mathrm{C} & \mathrm{C} & \mathrm{C} & \mathrm{C} \\
0 & 0 & 0 & 0 & 0 & 0 & 0 & 0 & 0 & 0 & 0 & 0 & 0 \\
0 & 0 & 0 & 0 & 0 & 0 & 0 & 0 & 0 & 0 & 0 & 0 & 0 \\
0 & 0 & 0 & 0 & 0 & 0 & 0 & 0 & 0 & 0 & 0 & 0 & 0 \\
\mathrm{f}(t ?) & \mathrm{f}(t ?) & \mathrm{f}(t ?) & 0 & \mathrm{C} & \mathrm{C} & \mathrm{f}(t ?) & \mathrm{f}(t ?) & 0 & \mathrm{f}(t ?) & 0 & 0 & 0 \\
\mathrm{f}(t ?) & \mathrm{f}(t ?) & \mathrm{f}(t ?) & \mathrm{C} & 0 & \mathrm{C} & \mathrm{f}(t ?) & \mathrm{f}(t ?) & 0 & \mathrm{f}(t ?) & 0 & 0 & 0 \\
\mathrm{f}(t ?) & \mathrm{f}(t ?) & \mathrm{f}(t ?) & \mathrm{C} & \mathrm{C} & 0 & \mathrm{f}(t ?) & \mathrm{f}(t ?) & 0 & \mathrm{f}(t ?) & 0 & 0 & 0
\end{array}\right]
$$


Close investigation of the potential time variant components posed in the product, $\mathbf{H}(\mathbf{t}) \mathbf{\Upsilon}(\mathbf{t})$, rewards a welcomed insight. For example, consider the multiplicative element formed in row ten, column one of the matrix above as

$$
\mathbf{E}_{(10,1)}=C+\mathrm{X}_{\dot{x}_{T_{o}}}(t) \mathrm{X}_{V_{T_{o}}}(t)+\mathrm{X}_{\dot{y}_{T_{o}}}(t) \mathrm{Y}_{V_{T_{o}}}(t)
$$

Using the coefficient expansions provided in Appendix D for $\mathrm{X}_{\dot{x}_{T_{o}}}(t)$ and $\mathrm{X}_{\dot{y}_{T_{o}}}(t)$ substitutions, Eq. (4.17) becomes

$$
\mathbf{E}_{(10,1)}=C-\cos \theta_{T_{o}} \cos \psi_{T_{o}}(t) \mathrm{X}_{V_{T_{o}}}(t)-\cos \theta_{T_{o}} \sin \psi_{T_{o}}(t) \mathrm{Y}_{V_{T_{o}}}(t)
$$

Again, from Appendix D, the coefficients $\mathrm{X}_{V_{T_{o}}}(t)$ and $\mathrm{Y}_{V_{T_{o}}}(t)$ are written as

$$
\begin{aligned}
& \mathrm{X}_{V_{T_{o}}}(t)=K_{1} \cos \psi_{T_{o}}(t)+K_{2} \sin \psi_{T_{o}}(t) \\
& \mathrm{Y}_{V_{T_{o}}}(t)=L_{1} \cos \psi_{T_{o}}(t)+L_{2} \sin \psi_{T_{o}}(t)
\end{aligned}
$$

where $K_{1}, K_{2}, L_{1}$, and $L_{2}$ represent time invariant constants gathered to a common term. Substituting Eq. (4.19) into Eq. (4.18),

$$
\begin{aligned}
\mathbf{E}_{(10,1)}= & C-\cos \theta_{T_{o}}\left[K_{1} \cos ^{2} \psi_{T_{o}}(t)+K_{2} \sin \psi_{T_{o}}(t) \cos \psi_{T_{o}}(t)\right. \\
& \left.+L_{1} \sin \psi_{T_{o}}(t) \cos \psi_{T_{o}}(t)+L_{2} \sin ^{2} \psi_{T_{o}}(t)\right]
\end{aligned}
$$

Rearranging the trigonometric terms,

$$
\begin{aligned}
\mathbf{E}_{(10,1)}= & C-\cos \theta_{T_{o}}\left[K_{1} \cos ^{2} \psi_{T_{o}}(t)+L_{2} \sin ^{2} \psi_{T_{o}}(t)\right. \\
& \left.+\left(K_{2}+L_{1}\right) \sin \psi_{T_{o}}(t) \cos \psi_{T_{o}}(t)\right]
\end{aligned}
$$

Inspection of Eq. (4.21) reveals the time variance posed by the $\psi_{T_{o}}(t)$ term will cancel if the following conditions hold:

$$
\begin{aligned}
& K_{1}=L_{2} \\
& K_{2}=-L_{1}
\end{aligned}
$$


which, if true would render Eq. (4.21) as

$$
\mathbf{E}_{(10,1)}=C-\cos \theta_{T_{o}}\left\{K_{1}\left[\cos ^{2} \psi_{T_{o}}(t)+\sin ^{2} \psi_{T_{o}}(t)\right]\right\}
$$

With the Pythagorean trigonometric identity of

$$
\cos ^{2} \psi_{T_{o}}(t)+\sin ^{2} \psi_{T_{o}}(t)=1
$$

and the assumption that Eq. (4.22) holds, $\mathbf{E}_{(10,1)}$ in Eq. (4.23) becomes a time invariant constant as

$$
\mathbf{E}_{(10,1)}=C-K_{1} \cos \theta_{T_{o}}
$$

Therefore, to prove time invariance in the element, Eq. (4.22) must hold true. Comparing the constants gathered in the formulation of Eq. (4.19) from Appendix D,

$$
\begin{gathered}
K_{1}=\cos \beta_{T} \sin \alpha_{T} \cos \phi_{T} \sin \theta_{T}+\sin \beta_{T} \sin \phi_{T} \sin \theta_{T}+\left.\cos \alpha_{T} \cos \beta_{T} \cos \theta_{T}\right|_{o} \\
L_{2}=\sin \beta_{T} \sin \phi_{T} \sin \theta_{T}+\cos \beta_{T} \sin \alpha_{T} \cos \phi_{T} \sin \theta_{T}+\left.\cos \alpha_{T} \cos \beta_{T} \cos \theta_{T}\right|_{o}
\end{gathered}
$$

exhibits $K_{1}=L_{2}$ and

$$
\begin{gathered}
K_{2}=\cos \beta_{T} \sin \alpha_{T} \sin \phi_{T}-\left.\sin \beta_{T} \cos \phi_{T}\right|_{o} \\
L_{1}=\sin \beta_{T} \cos \phi_{T}-\left.\cos \beta_{T} \sin \alpha_{T} \sin \phi_{T}\right|_{o}
\end{gathered}
$$

exhibits $K_{2}=-L_{1}$ satisfying Eq. (4.22) which proves $\mathbf{E}_{(10,1)}$ is not a function of $\psi_{T_{o}}(t)$ and simplifies to a time invariant constant.

The remaining elements in $\mathbf{E}$ of Eq. (4.16) are proven to be independent of time with an equivalent procedure described above in the row ten, column one analysis. Understanding the source or reason for this result requires a broader matrix-level view of the EOM presented in Chapter 2. In hindsight, the direct substitution of the nonlinear tanker translational kinematics in Eq. (2.3) into the nonlinear receiver translational kinematics 
in Eq. (2.1) prior to linearization, and without the wind terms, results in a time invariant receiver disturbance matrix, $\mathbf{H}$. However, this step will modify the receiver disturbance vector, $\underline{w}$, (to include the tanker translational velocity components with respect to the tanker wind frame rather than the inertial frame) which was initially assumed fixed as a given problem description in the original research objective. Future work should consider this simplification as recommended in Chapter 6 .

In summary, the time variance in the $\mathbf{H}(\mathbf{t})$ and $\mathbf{\Upsilon}(\mathbf{t})$ matrices conveniently dissipate in the product, and the constant resultant matrix allows the problem to be formulated in a manner suitable for application of (i) the Friedland disturbance rejection control law, (ii) the existing linear time invariant LQR MIMO $[1,9,10]$ structure, and (iii) utilization of the existing definition for the tanker state vector, $\underline{x}_{T_{A}}$. Reposing the receiver open loop state-space system now as time invariant as previously described in Eq. (2.14),

$$
\underline{\dot{x}}=\mathbf{A} \underline{x}+\mathbf{B} \underline{u}+\mathbf{E} \underline{x}_{T_{A}}
$$

where $\mathbf{E}$ represents the time invariant product, $\mathbf{H}(\mathbf{t}) \mathbf{\Upsilon}(\mathbf{t})$. Refer to Fig. (4.1) for the block diagram depiction of Eq. (4.28) as well as the tanker closed loop system in Eq. (2.15). Note the asterisk on the $\mathbf{C}$ receiver output is used to indicate a sparse matrix with only the final three columns populated as a $3 \times 3$ identity square, i.e., relative position between the two vehicles for the integral control law. 


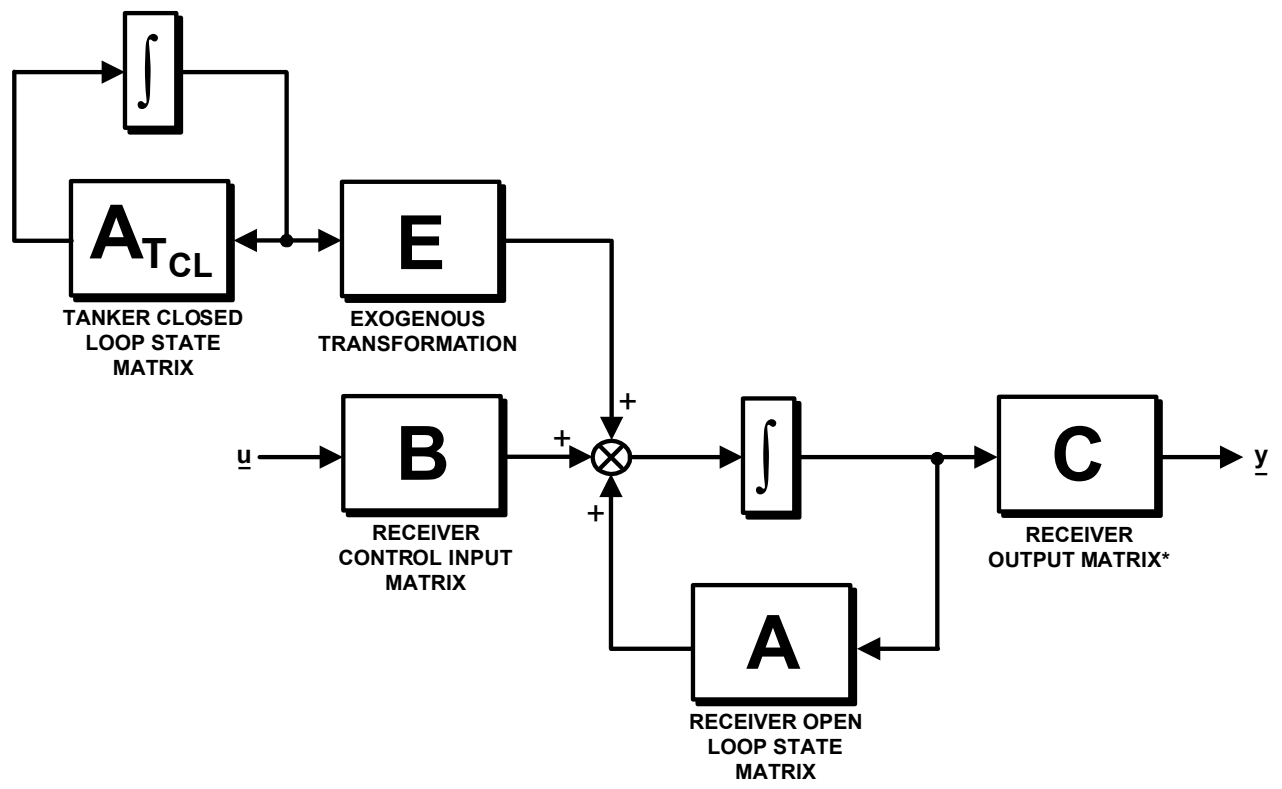

Figure 4.1. Receiver Open Loop LTI State-Space System. 


\section{CHAPTER 5}

\section{SIMULATION RESULTS}

This chapter presents results from two simulation environments, a linear model and a nonlinear model. The first section describes the linear model which is used in a Single Input Single Output (SISO) classical analysis of the receiver vehicle for observing both step responses in the time domain and Bode plots in the frequency domain. A trade study is conducted on multiple performance indices, which are later employed into the nonlinear model (by incorporation of the Kalman gain sets generated with each performance index in evaluation). The second simulation environment, discussed in the subsequent section of this chapter, presents results for the high fidelity nonlinear model. This simulation employs the full Linear Quadratic Regulator Multiple Input Multiple Output (LQR MIMO) control architecture on both the receiver and tanker vehicle and includes dynamic modeling of the aerodynamic coupling between the two vehicles, as well as the inertial winds of the atmosphere.

\subsection{Linear Model}

A linear model construction allows for rapid preliminary result generation and expedited comparison between control law architectures. Refer to Fig. (5.1) which depicts the closed loop system for both the LQR MIMO $[1,9,10]$ controller and the Friedland control law. Due to the distinct form of Eq. (3.29), the absence of the $\mathbf{K}_{\mathbf{o}_{\mathbf{T}}}$ path yields the existing control law methodology in place. The Friedland improvement is enabled solely via this feed-forward path from the tanker state equation alone, where, per Eq. (2.22), 
$\mathbf{K}_{\mathbf{o}_{\mathbf{T}}}=-\mathbf{R}^{-\mathbf{1}} \mathbf{B}^{\mathbf{T}} \overline{\mathbf{M}}_{\mathbf{2}}$ represents the new gain to be summed into the existing LQR MIMO $[1,9,10]$ controller.

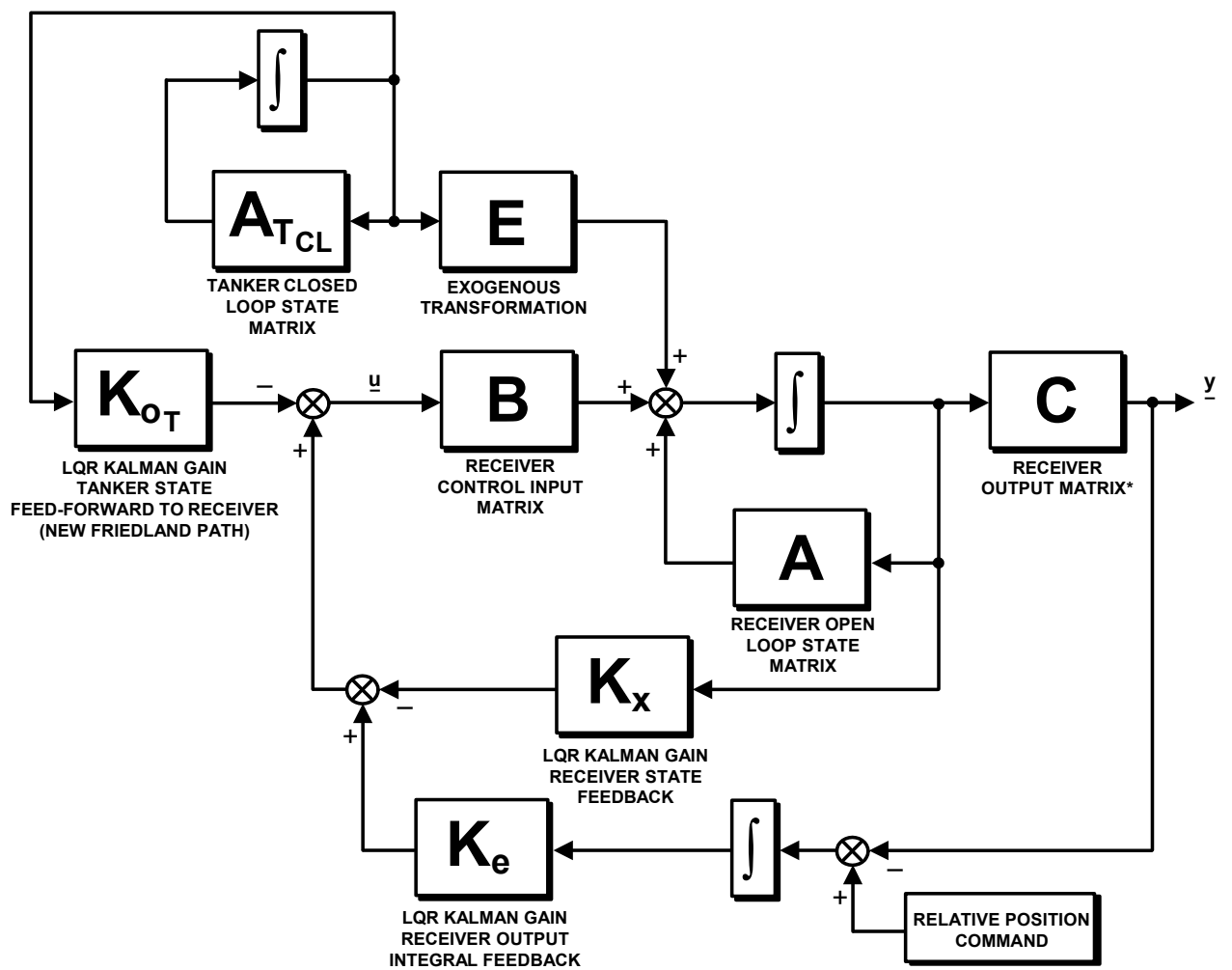

Figure 5.1. Receiver Closed Loop LTI State-Space System.

The solution to the infinite horizon linear quadratic regulator problem is generated from the augmented metastate-space system as depicted in the block diagram Fig. (5.2) and represents Eq. (3.28). Next, the Kalman steady state gains are extracted for use in the expanded architecture shown previously in the expanded block diagram in Fig. (5.1), which is consistent with the control law presented in Eq. (3.29), and allows formulation of a SISO linear analysis from the tanker bank attitude command path as the input and the receiver lateral y-deviation from the refueling position as the output. For the purpose of figure brevity, the single input tanker attitude command path is not explicitly depicted 
as a result of containment within the closed loop tanker system. Actual linear model results are generated with the fully expanded model. Additionally, the output pick off point of receiver lateral y-deviation stems from the second $\mathbf{C}$ metastate-space system output.

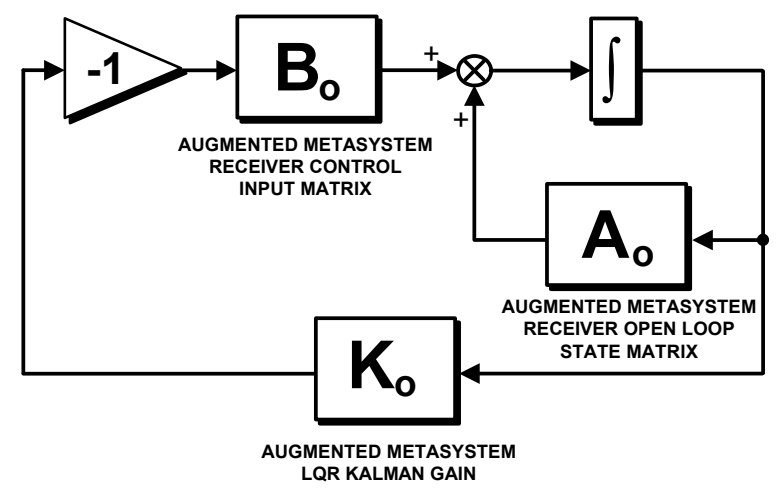

Figure 5.2. Augmented Receiver Closed Loop LTI Metastate-Space System.

The employment of the LQR MIMO $[1,9,10]$ control law into the nonlinear simulation utilizes a Lagrange interpolation scheme between six tanker nominal conditions as described in Table 5.1 [1]. Therefore, the SISO linear analysis is repeated for each nominal condition.

Table 5.1. Nominal Conditions by Turn Rate and Airspeed of the Tanker

\begin{tabular}{|c|c|c|}
\hline Nominal Condition & Turn Rate $(\mathrm{deg} / \mathrm{s})$ & Airspeed $(\mathrm{m} / \mathrm{s})$ \\
\hline \hline 1 & $\dot{\psi}_{T, 1}=0$ & $V_{T, 1}=190$ \\
\hline 2 & $\dot{\psi}_{T, 1}=0$ & $V_{T, 2}=210$ \\
\hline 3 & $\dot{\psi}_{T, 2}=1.7$ & $V_{T, 1}=190$ \\
\hline 4 & $\dot{\psi}_{T, 2}=1.7$ & $V_{T, 2}=210$ \\
\hline 5 & $\dot{\psi}_{T, 3}=-1.7$ & $V_{T, 1}=190$ \\
\hline 6 & $\dot{\psi}_{T, 3}=-1.7$ & $V_{T, 2}=210$ \\
\hline
\end{tabular}


To begin the comparison of the existing LQR MIMO $[1,9,10]$ performance with the addition of the Friedland disturbance rejection control law, the Kalman gains are generated for the augmented metastate-space system (Fig. (5.1)) at the six nominal conditions with unity weights in the performance index. Both state and control action penalty matrices, $\mathbf{Q}_{x}$ and $\mathbf{R}$, respectively, are set to identity matrices. Recall the state penalty matrix $\mathbf{Q}_{x}$ corresponds to the receiver states alone. The metastate penalty matrix, $\mathbf{Q}$, appends the receiver state penalty matrix, $\mathbf{Q}_{x}$, with zeroes as discussed in Chapter 2 due to the fact that the uncontrollable tanker states are not penalized in the cost minimization. The other weights, set to one, reflect an even distribution of penalty across both receiver states and control actions. The following Eq. (5.1) presents the unity performance index and is evaluated for all six nominal conditions where diag denotes the diagonal components of the square cost matrices, $\mathbf{Q} \in \Re^{28 x 28}$ and $\mathbf{R} \in \Re^{6 x 6}$

$$
\begin{aligned}
& \mathbf{Q}=\operatorname{diag}\left[\begin{array}{llllllllllllllllllllllllllll}
1 & 1 & 1 & 1 & 1 & 1 & 1 & 1 & 1 & 1 & 1 & 1 & 1 & 1 & 1 & 0 & 0 & 0 & 0 & 0 & 0 & 0 & 0 & 0 & 0 & 0 & 0 & 0
\end{array}\right] \\
& \mathbf{R}=\operatorname{diag}\left[\begin{array}{llllll}
1 & 1 & 1 & 1 & 1 & 1
\end{array}\right]
\end{aligned}
$$

Two SISO figures are generated with a subfigure per nominal condition. The first is a time history of the receiver relative positional y-lateral deviation from the refueling station due to a tanker bank attitude command step response. The blue time history with circle markers represent the existing LQR MIMO $[1,9,10]$ structure in place (although with newly generated Kalman gains as aforementioned) and the green square marked lines represent the equivalent structure with the addition of the Friedland disturbance rejection path. The second figure type presented below are frequency response Bode plots, depicting both magnitude $(\mathrm{dB})$ and phase angle (deg) of the equivalent SISO relationship used in the time history. Reiterating, the output is the receiver y-lateral deviation now due to a sinusoidal frequency sweep via the tanker bank attitude command path input to the metastate-space system. 
Fig. (5.3) clearly exhibits a marketable improvement with the Friedland control law active where all six nominal conditions show significant increase in damping. Peak lateral errors from the refueling position are reduced $750 \%$ from $8 \mathrm{e}-3$ meters $(0.026 \mathrm{ft})$ to less than 1e-3 meters $(0.003 \mathrm{ft})$. However, as will be shown in the nonlinear model simulation results in the following section, the price is expensive for this performance in terms of the control action and therefore unrealistic. This initial result simply exhibits the potential improvement to the existing LQR MIMO $[1,9,10]$ with the Friedland feed-forward compensation in an ideal circumstance with excess control power available (unity $\mathbf{R}$ provides evenly distributed penalty for all effectors). Despite the generous circumstance which assumes control power margin, two additional attributes are noted within these time histories. One, the existing LQR MIMO $[1,9,10]$ control law exhibits a subtle steady state error due to a tanker bank in nominal conditions three through six despite being a type one system. Second, the transient response settling time with the Friedland compensation enabled appears to reside from approximately 50 to 70 seconds. Therefore, as the control action is further penalized in the forthcoming analysis (with performance indices utilizing increased weight in $\mathbf{R}$ to account for actual control power available), one would not expect a reduction in this settling time transitory period. However, upon further linear analysis, the minimum settling time of 50 to 70 seconds for the ideal performance index with the Friedland compensation active may be improved by modifying the feedforward command dynamics in the tanker bank attitude controller. Two first order .10 rps lags in place for preventing tanker effector saturation, in response to a step bank attitude command, appear to dominate the maximum bandwidth achievable for receiver station-keeping control. This topic is also referenced as a potential future investigative effort in Chapter 6.

Fig. (5.4) depicts the Bode frequency response of the system with equivalent control law gains generated by unity $\mathbf{Q}$ and $\mathbf{R}$ performance index weights. Readily apparent is 

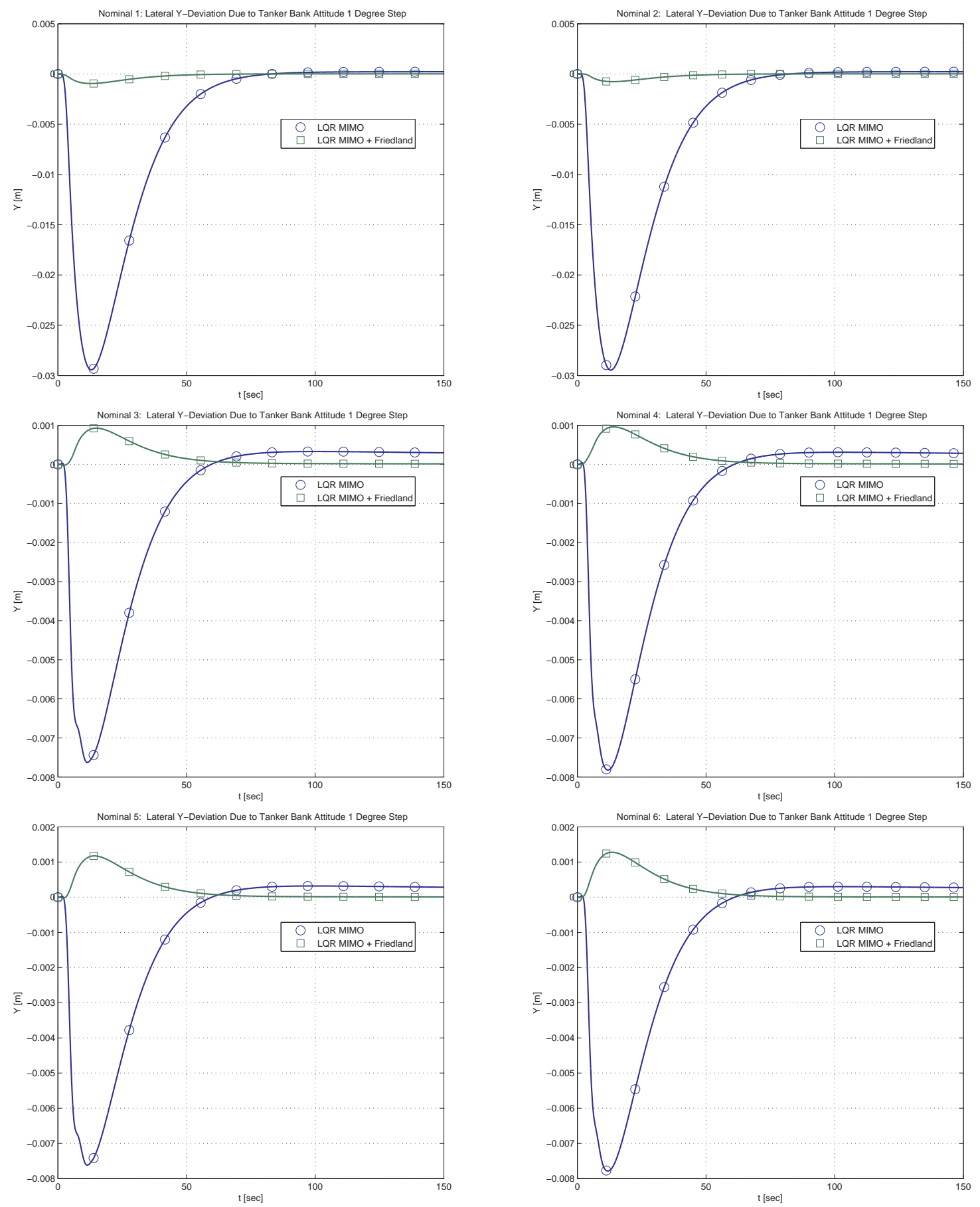

Figure 5.3. Receiver Lateral (y) Deviation Response Due to Tanker Bank Attitude Command Unit Step [m/deg] per Nominal Condition (Unity Weight Q, R). 
the 20-30 dB reduction in magnitude response offered by the Friedland control law over the baseline from steady state to approximately $4 \mathrm{rps}$. Above these frequencies, the differences between the controllers converge due to operating frequencies beyond the rigid body bandwidth of the total metasystem. Regarding the phase response presented in Fig. (5.4), all nominal conditions indicate a significant increase in phase angle across the frequency spectrum with the Friedland control law enabled. This result is indicative of more lead in the system in that the Friedland control law increases the receiver proactive (feed-forward) response due to the accelerating tanker.

Moving beyond the assumption of perfect control power, the Kalman gains are now recomputed at each nominal condition using the current performance index definitions $(\mathbf{Q}, \mathbf{R})$ in place for the existing $\operatorname{LQR}$ MIMO $[1,9,10]$ controller. These results are expected to be more representative of actual performance improvements in the nonlinear system as the performance index weights were derived in previous work to prevent control saturation while optimizing the station-keeping task. Two baseline performance indices are used in order to schedule between the nominal conditions involving level and turning flight conditions for the tanker. The level flight baseline performance index (nominal conditions one and two) is

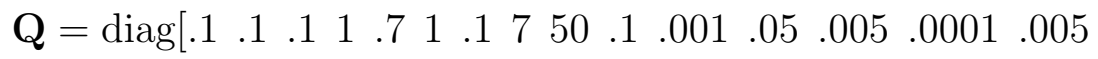

$$
\begin{aligned}
& \left.\begin{array}{lllllllllllll}
0 & 0 & 0 & 0 & 0 & 0 & 0 & 0 & 0 & 0 & 0 & 0 & 0
\end{array}\right] \\
& \mathbf{R}=\operatorname{diag}\left[\begin{array}{llllll}
25 & 10 & 15 & 1200 & 5000000 & 5000000
\end{array}\right]
\end{aligned}
$$



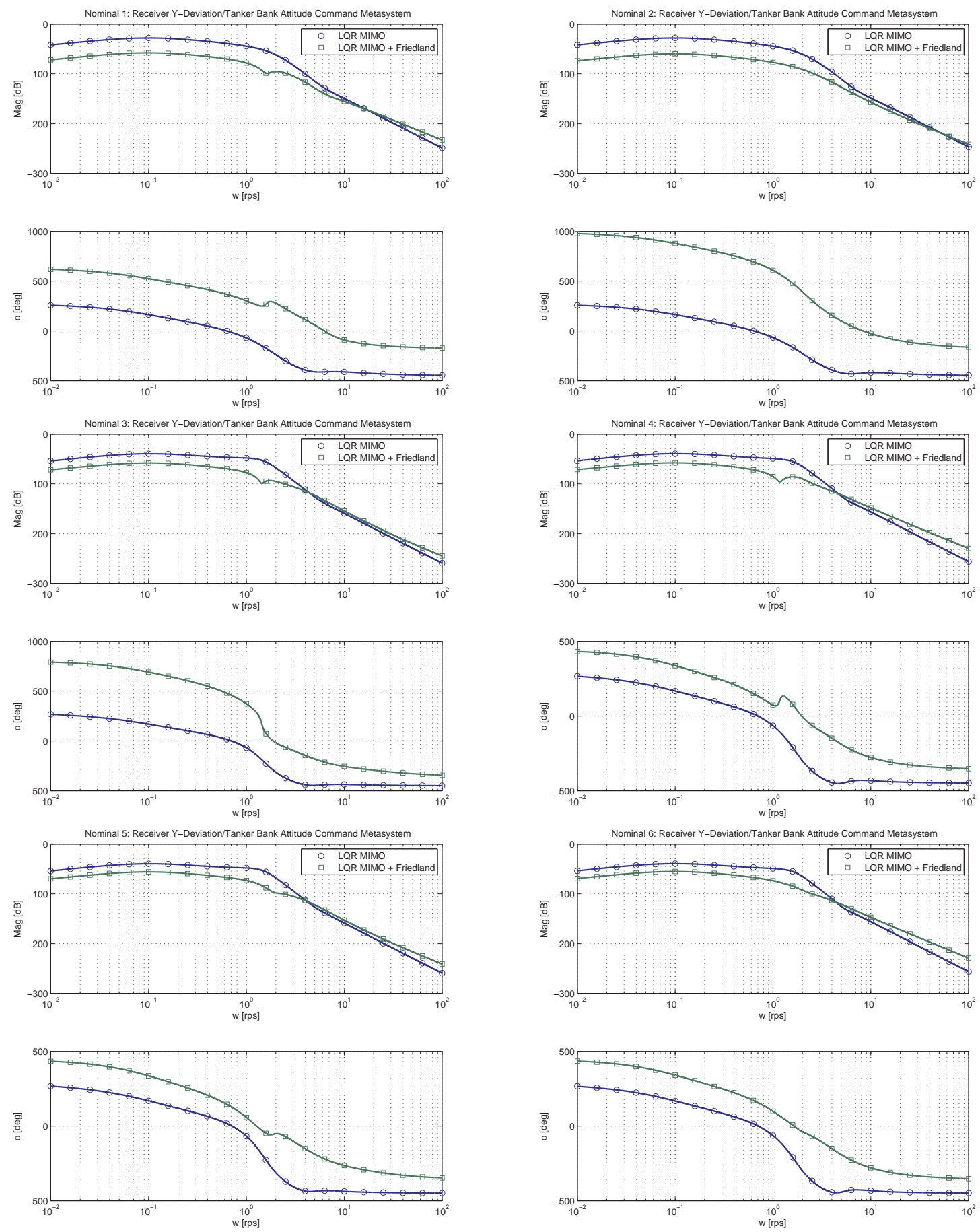

Figure 5.4. Receiver Lateral (y) Deviation to Tanker Bank Attitude Command Metasystem Frequency Response per Nominal Condition (Unity Weight Q, R). 
and the turning flight baseline performance index (nominal conditions three through six) is

$$
\begin{aligned}
& \mathbf{Q}=\operatorname{diag}[.01100 .0110 .51001001107 e-6 \quad 1 e-67 e-65 e-85 e-85 e-8 \\
& \left.\begin{array}{lllllllllllll}
0 & 0 & 0 & 0 & 0 & 0 & 0 & 0 & 0 & 0 & 0 & 0 & 0
\end{array}\right] \\
& \mathbf{R}=\operatorname{diag}\left[\begin{array}{lllll}
5 & .1 & 2 & 12005000000 & 5000000
\end{array}\right]
\end{aligned}
$$

Given that many of the effectors and states possess different units, a simple sort operation on the weighted components does not instantly reveal the ultimate control law priority provided by the performance index. However, for the purpose of a quick insight in presenting each performance index in evaluation, the sort output is listed and includes variable units. Consideration is required on the type of units on the variable at question in order to determine priority relative to another variable. Apparently, the baseline control law prioritizes the use of the receiver elevators (deg) as the primary effectors for all nominal conditions (level and turning flight). Thereafter, the selected control effectors of the receiver are weighted in the following order from highest to lowest utilization priority: rudder (deg), aileron (deg), thrust $(\mathrm{N})$, thrust vectoring about the $y$ body axis (deg), and thrust vectoring about the $z$ body axis (deg). (Note in this specific application with a Learjet as the receiver vehicle, thrust vectoring does not exist. Rather than re-tailoring the input vector in the controller formulation, the thrust vectoring actions are weighted significantly higher than the other effectors to prevent use). The control law does not prioritize receiver state deviation in an equivalent manner for level and turning flight however. Sorting the weights of the $\mathbf{Q}$ matrix reveals the receiver control law will attempt to prioritize state deviations (from the nominal operating condition) in the following order for level flight from the most allowed deviation to the most penalized deviation: integral error of lateral deviation $e_{y}(-), \Delta y$ lateral deviation $(\mathrm{m})$, integral error of axial deviation $e_{x}(-)$, integral error of height deviation $e_{z}(-), \Delta z$ deviation $(\mathrm{m}), \Delta V$ 
velocity deviation (m/s), $\Delta \beta$ sideslip deviation (deg), $\Delta \alpha$ angle of attack deviation (deg), $\Delta \psi$ relative heading deviation (deg), $\Delta x$ axial deviation $(\mathrm{m}), \Delta q$ pitch rate deviation $(\mathrm{deg} / \mathrm{s}), \Delta p$ roll rate deviation $(\mathrm{deg} / \mathrm{s}), \Delta r$ yaw rate deviation $(\mathrm{deg} / \mathrm{s}), \Delta \theta$ relative pitch deviation (deg), and finally the $\Delta \phi$ relative roll deviation (deg). For turning flight, the receiver control law prioritizes the state deviations as follows (again from lowest to highest penalty): integral error of axial deviation $e_{x}(-)$, integral error of lateral deviation $e_{y}(-$ ), integral error of height deviation $e_{z}(-), \Delta y$ lateral deviation (m), $\Delta x$ axial deviation (m), $\Delta z$ height deviation $(\mathrm{m}), \Delta V$ velocity deviation $(\mathrm{m} / \mathrm{s}), \Delta \alpha$ angle of attack deviation (deg), $\Delta q$ pitch rate deviation $(\mathrm{deg} / \mathrm{s}), \Delta \theta$ relative pitch deviation (deg), $\Delta p$ roll rate deviation (deg/s), $\Delta \phi$ relative roll deviation (deg), $\Delta \beta$ sideslip deviation (deg), $\Delta r$ yaw rate deviation (deg/s), and finally the $\Delta \psi$ relative heading deviation (deg).

Refer to Fig. (5.5) for a direct comparison between the existing LQR MIMO $[1,9,10]$ controller and one with Friedland compensation enabled. Again, consistent with the ideal linear analysis (unity performance index), the Friedland compensation offers considerable improvement for receiver station-keeping amidst a tanker bank maneuver. Peak lateral errors observed in the step response time histories are reduced approximately $215 \%$ from the worst case profile (nominal condition six) at $14 \mathrm{~m}(45.9 \mathrm{ft})$ to nearly $6.5 \mathrm{~m}(21.3$ ft) deviation. The steady state error with the existing LQR MIMO [1,9,10] structure is now grossly apparent for nominal conditions three through six with these optimized gains and is corrected with the Friedland compensation. Unfortunately, due to the less invasive control power scheme with the performance index, the time duration of the plots are now extended to 500 seconds to allow for extremely slow settling times. Although significant improvement is observed for both the steady state error and the transient response with the Friedland compensation, a settling time of 300 seconds with a $6.5 \mathrm{~m}(21.3 \mathrm{ft})$ lateral refueling deviation is clearly unacceptable. This response is addressed in the final linear analysis results with a revised performance index. 

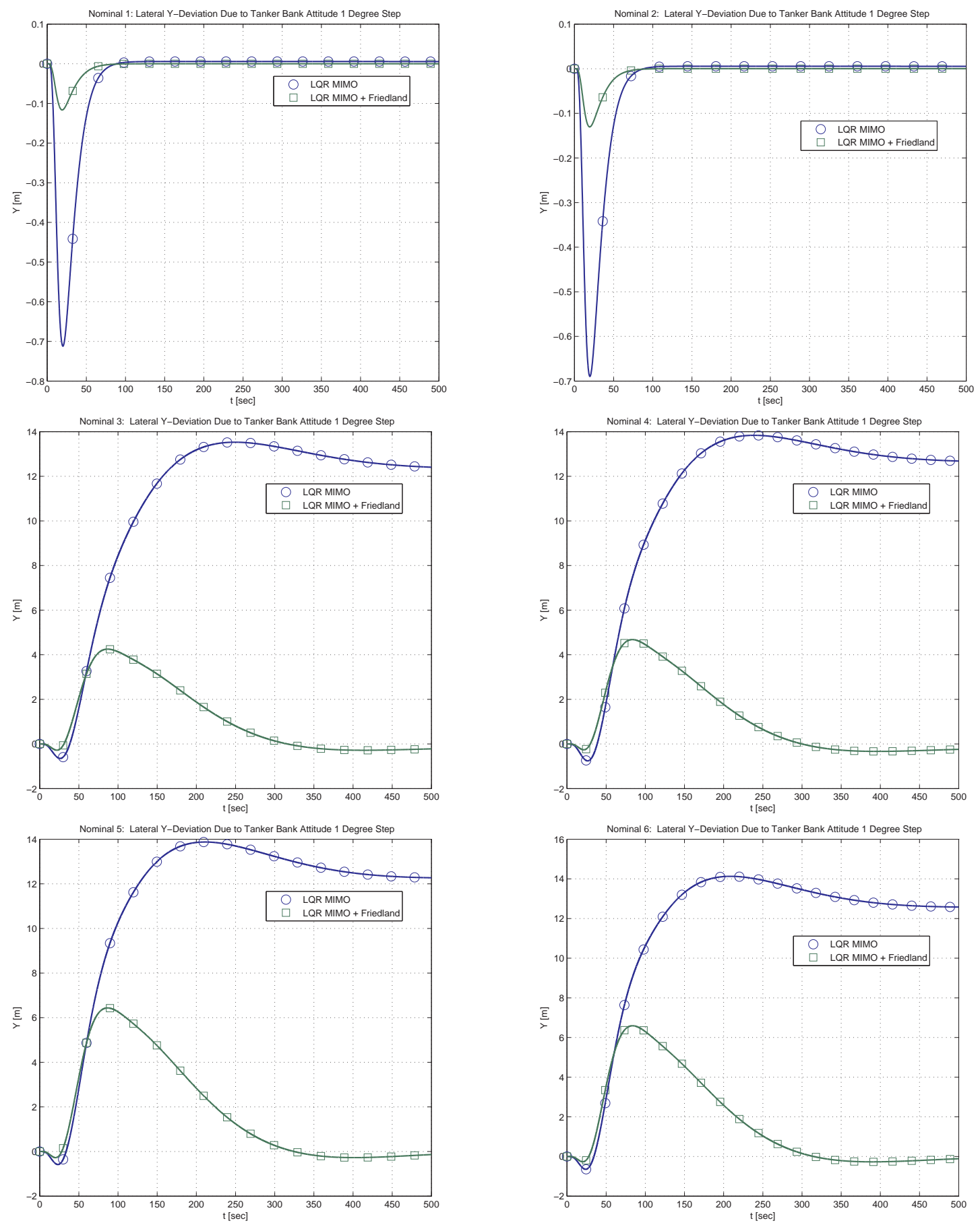

Figure 5.5. Receiver Lateral (y) Deviation Response Due to Tanker Bank Attitude Command Unit Step [m/deg] per Nominal Condition (Currently Employed Q, R). 
Fig. (5.6) repeats the Bode frequency response plots for the receiver lateral ydeviation in response to a tanker bank attitude command input path for the current performance index in place. Consistent with the time histories, the disturbance magnitude attenuation is significantly improved with the new control law for nominal conditions one and two with nearly a $25 \mathrm{~dB}$ gain reduction from steady state to approximately 2 rps. In the remaining nominal conditions, the impact of the Friedland compensation is lessened considerably with only a 5 to $10 \mathrm{~dB}$ reduction for frequencies below $20^{-2} \mathrm{rps}$. Above this frequency, disturbance attenuation is minimal. Note below $15^{-1} \mathrm{rps}$, the current performance index generates two controllers that effectively amplify the disturbance. This result is in agreement with the time history observation that a 1 degree bank step unacceptably results in a $14 \mathrm{~m}$ (45.9 ft) deviation. The phase lead offered by the Friedland compensation in Fig. (5.6) with the baseline performance index is not as dramatic as observed with the unity performance index. In fact, the first nominal condition indicates a greater phase angle with the Friedland compensation disabled for frequencies up to 40 rps with this gain set.

Finally, the performance index is revised to correct the poor linear responses (settling time and disturbance attenuation, or lack thereof) encountered above with the existing $\mathbf{Q}$ and $\mathbf{R}$ weights. The refined performance index for level flight (nominal conditions one and two) is

$$
\begin{aligned}
& \mathbf{Q}=\operatorname{diag}\left[\begin{array}{llllllllllllll}
10 & 10 & 10 & 100 & 70 & 100 & 10 & 700 & 5000 & 10 & 20 & 5 & 2.5 & 25
\end{array}\right. \\
& \left.\begin{array}{lllllllllllll}
0 & 0 & 0 & 0 & 0 & 0 & 0 & 0 & 0 & 0 & 0 & 0 & 0
\end{array}\right] \\
& \mathbf{R}=\operatorname{diag}\left[\begin{array}{llllll}
25 & 10 & 15 & 1200 & 5000000 & 5000000
\end{array}\right]
\end{aligned}
$$



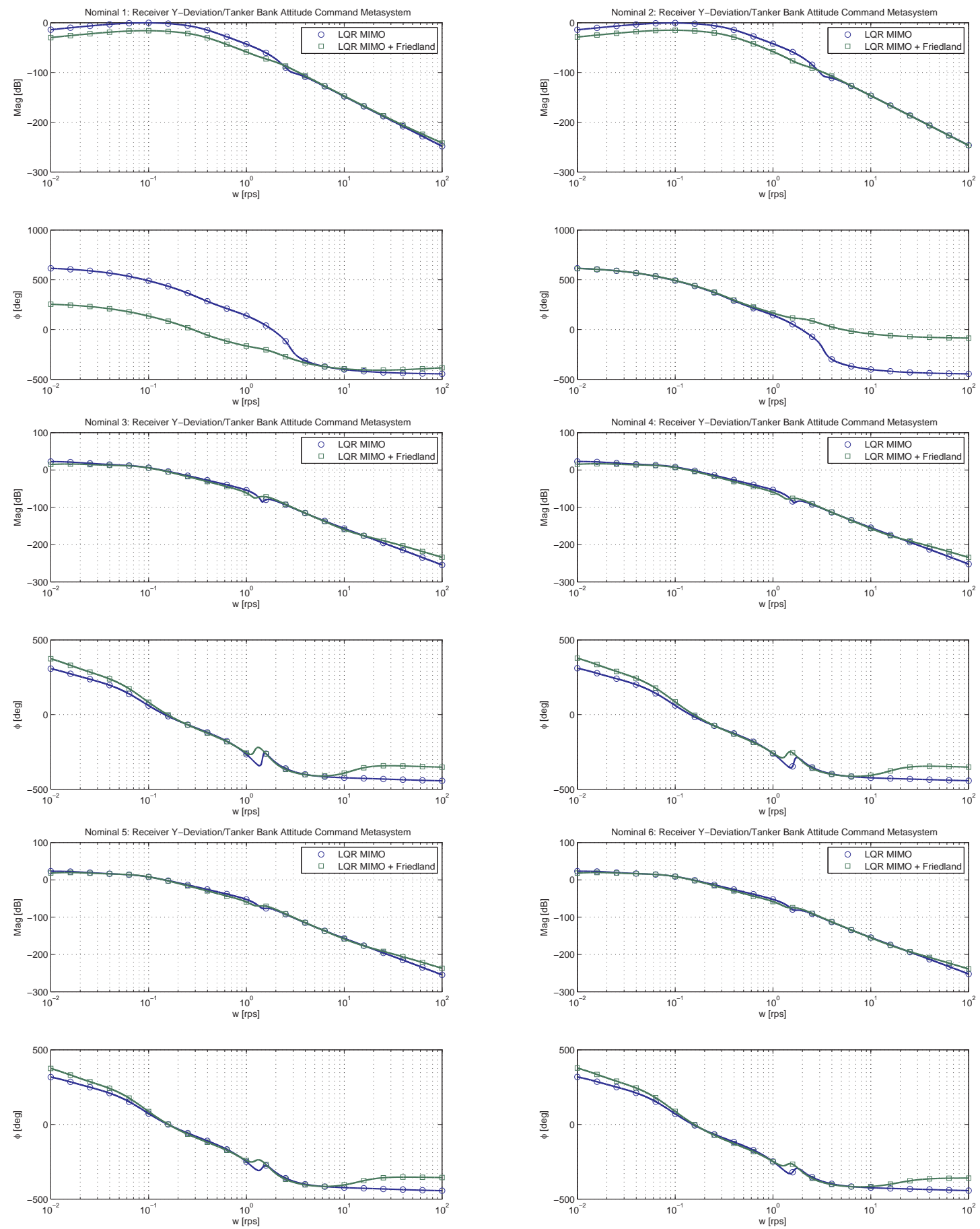

Figure 5.6. Receiver Lateral (y) Deviation to Tanker Bank Attitude Command Metasystem Frequency Response per Nominal Condition (Currently Employed Q, R). 
and the turning flight refined performance index (nominal conditions three through six) is

$$
\begin{aligned}
& \mathbf{Q}=\operatorname{diag}[1100001100050100001000010010007 e-4.027 e-42.5 e-51 e-4
\end{aligned}
$$

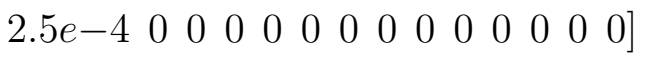

$$
\begin{aligned}
& \mathbf{R}=\operatorname{diag}\left[\begin{array}{lllll}
5 & .1 & 2 & 12005000000 & 5000000
\end{array}\right]
\end{aligned}
$$

Inspection of the refined performance indices reveals the receiver effector apparent priorities remain equivalent to the baseline control law with the elevators (deg) as the primary effectors followed by the rudder (deg), aileron (deg), thrust (N), thrust vectoring about the $y$ body axis (deg), and thrust vectoring about the $z$ body axis (deg) for all nominal conditions (level and turning flight). (Again the specific Learjet receiver vehicle studied in this application does not employ thrust vectoring and is disabled by utilizing significantly higher weights in the control action penalty matrix, $\mathbf{R}$ ). The refined performance index does prioritize the receiver state deviations differently from the baseline control law however. In the refined controller, the apparent priority sort is as follows from the least penalized to greatest penalized deviation for level flight: integral error of lateral deviation $e_{y}(-)$, integral error of axial deviation $e_{x}(-), \Delta z$ height deviation $(\mathrm{m}), \Delta V$ velocity deviation $(\mathrm{m} / \mathrm{s}), \Delta \beta$ sideslip deviation (deg), $\Delta \alpha$ angle of attack deviation (deg), $\Delta \psi$ relative heading deviation (deg), $\Delta x$ axial deviation $(\mathrm{m}), \Delta y$ lateral deviation $(\mathrm{m})$, integral error of height deviation $e_{z}(-), \Delta q$ pitch rate deviation (deg/s), $\Delta p$ roll rate deviation $(\mathrm{deg} / \mathrm{s}), \Delta r$ yaw rate deviation $(\mathrm{deg} / \mathrm{s}), \Delta \theta$ relative pitch deviation $(\mathrm{deg})$, and finally the $\Delta \phi$ relative roll deviation (deg). For turning flight, the receiver control law apparent priority sort for state deviations from lowest to highest penalty is as follows: integral error of axial deviation $e_{x}(-)$, integral error of lateral deviation $e_{y}(-)$, integral error of height deviation $e_{z}(-), \Delta x$ axial deviation $(\mathrm{m}), \Delta z$ height deviation $(\mathrm{m}), \Delta y$ lateral deviation $(\mathrm{m}), \Delta V$ velocity deviation $(\mathrm{m} / \mathrm{s}), \Delta \alpha$ angle of attack deviation (deg), 
$\Delta q$ pitch rate deviation $(\mathrm{deg} / \mathrm{s}), \Delta \theta$ relative pitch deviation (deg), $\Delta p$ roll rate deviation (deg/s), $\Delta \phi$ relative roll deviation (deg), $\Delta \beta$ sideslip deviation (deg), $\Delta r$ yaw rate deviation $(\mathrm{deg} / \mathrm{s})$, and finally the $\Delta \psi$ relative heading deviation (deg).

Reference Fig. (5.7) for the time history receiver y-deviation responses due to a tanker bank step command with the newly generated Kalman gains (using the revised performance index). All six nominal conditions demonstrate superior performance with the Friedland compensation. Also, the responses do not amplify the input as previously discussed and settle within acceptable durations.

Fig. (5.8) asserts these results with the newly revised performance index in the frequency domain. Similarly to the baseline performance index, nominal conditions one and two dominate in disturbance magnitude attenuation at approximately $25 \mathrm{~dB}$ in difference over the metasystem rigid body bandwidth, also consistent with the time histories for this gain set. Again, the remaining nominal conditions exhibit lessened impact of the Friedland compensation, but the distinguishing point from the prior (currently employed) performance index resides at a frequency of $10^{-1} \mathrm{rps}$. Below this frequency, the benefits of the new control law begin to show as the Friedland compensation magnitude response monotonically decreases to approximately $25 \mathrm{~dB}$ of attenuation at steady state. Interestingly, the revision of the performance index alone optimized the Friedland control law benefits on the low frequency decade observed in Fig. (5.8) between $10^{-2}$ rps and $10^{-1} \mathrm{rps}$. Additionally, as observed in time history form, the performance index revision improves the existing LQR MIMO $[1,9,10]$ controller and now exhibits unity gain at these low frequencies rather than amplification. Finally, the refined performance index depicts an increase in phase angle response with the Friedland control law enabled as previously observed in the unity performance index results. Consistent with the magnitude differences between the two controllers for the remaining nominal conditions (turning flight), the phase angle lead offered by the Friedland control law is lessened compared to the 
level flight nominal conditions. This result is considered directly attributable to the fact that two performance indices are being evaluated across all six nominal conditions (level and turning). 

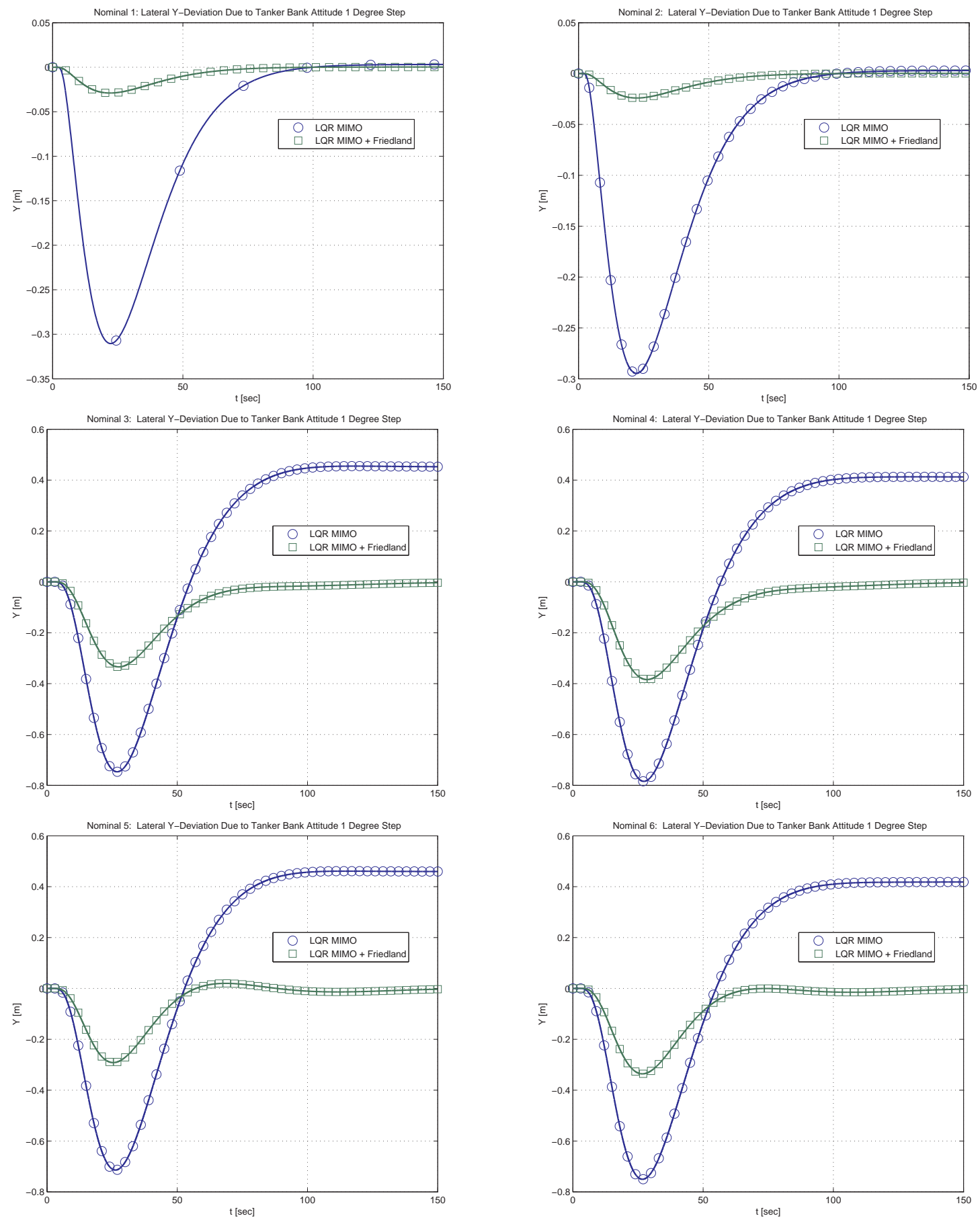

Figure 5.7. Receiver Lateral (y) Deviation Response Due to Tanker Bank Attitude Command Unit Step [m/deg] per Nominal Condition (Revised Weight Q, R). 

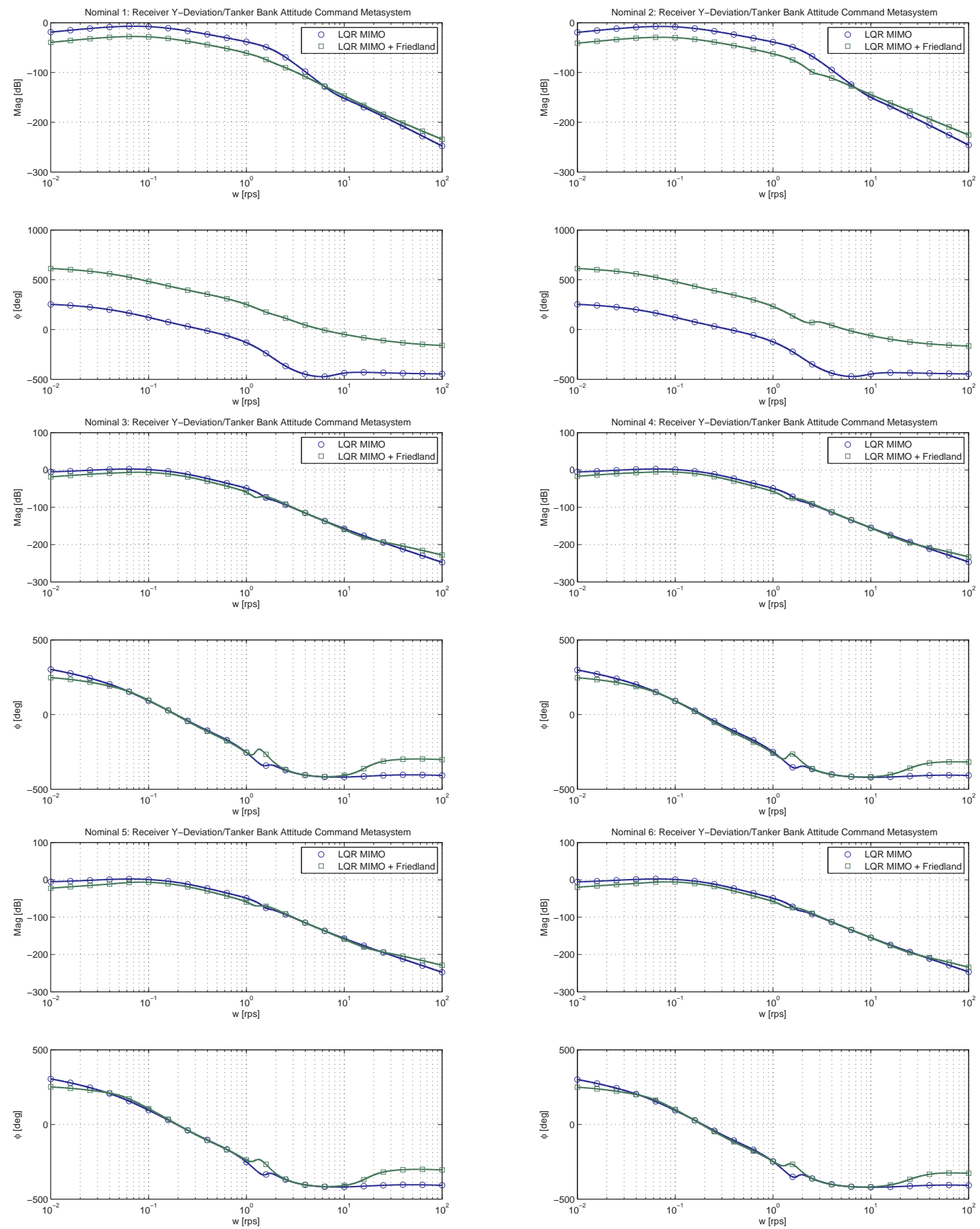

Figure 5.8. Receiver Lateral (y) Deviation to Tanker Bank Attitude Command Metasystem Frequency Response per Nominal Condition (Revised Weight Q, R). 


\subsection{Nonlinear Model}

The control law feedback and feed-forward gains, computed using the linear model as previously discussed, are extracted for direct use in the nonlinear model, referenced in Fig. (5.9). Note the state-space matrices are no longer used in the high fidelity simulation, and the nonlinear blocks pertain to the EOM presented in detail in Chapter 2. Therefore, in order for good correlation between the linear and nonlinear results regarding stability and performance, an accurate representation in the initial linearized state-space matrices is imperative. However, depending on the robustness of the controller, some deviation between the two (linear and nonlinear) may be tolerated. Note in this section, additional disturbances (wind) will be discussed and included in the simulation results. These effects were not included in the linear control law formulation in this research and are recommended to be considered in future work in Chapter 6.

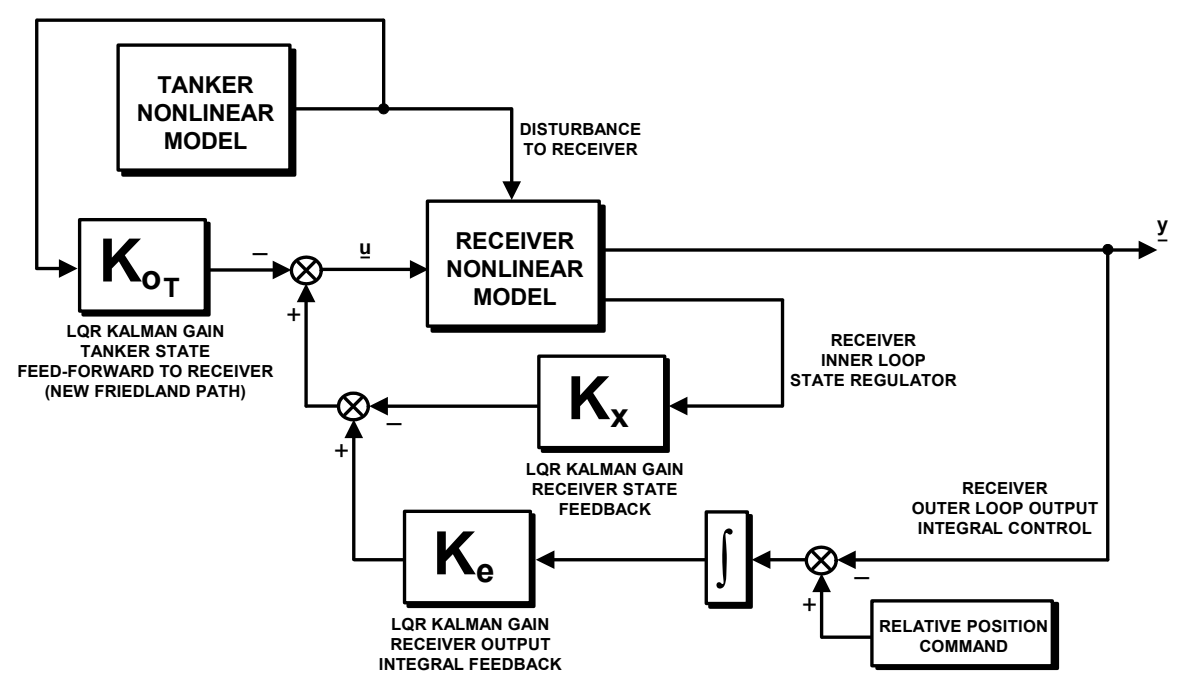

Figure 5.9. Receiver Closed Loop Nonlinear System. 


\subsubsection{Control Law Analysis in the Presence of Tanker Flow Field}

Employment of the controllers into the full order nonlinear simulation confirms both performance results observed in the linear analysis as well as expectations of control power saturation for ideal $\mathbf{Q}, \mathbf{R}$ weights set as unity in the performance index. Additionally, the full simulation now includes the effect of the tanker wake-vortex induced wind [16-19] on the receiver while behind the tanker. However, the prevailing wind and turbulence are disabled at this point in the analysis. The subsequent portion of this chapter will enable all known external disturbances on the receiver including these inertial atmospheric effects. For the initial analysis of the controllers employed into the nonlinear simulation, the station-keeping task is to maintain refueling contact position while the tanker performs a 30 degree bank maneuver as depicted in Fig. (5.10).

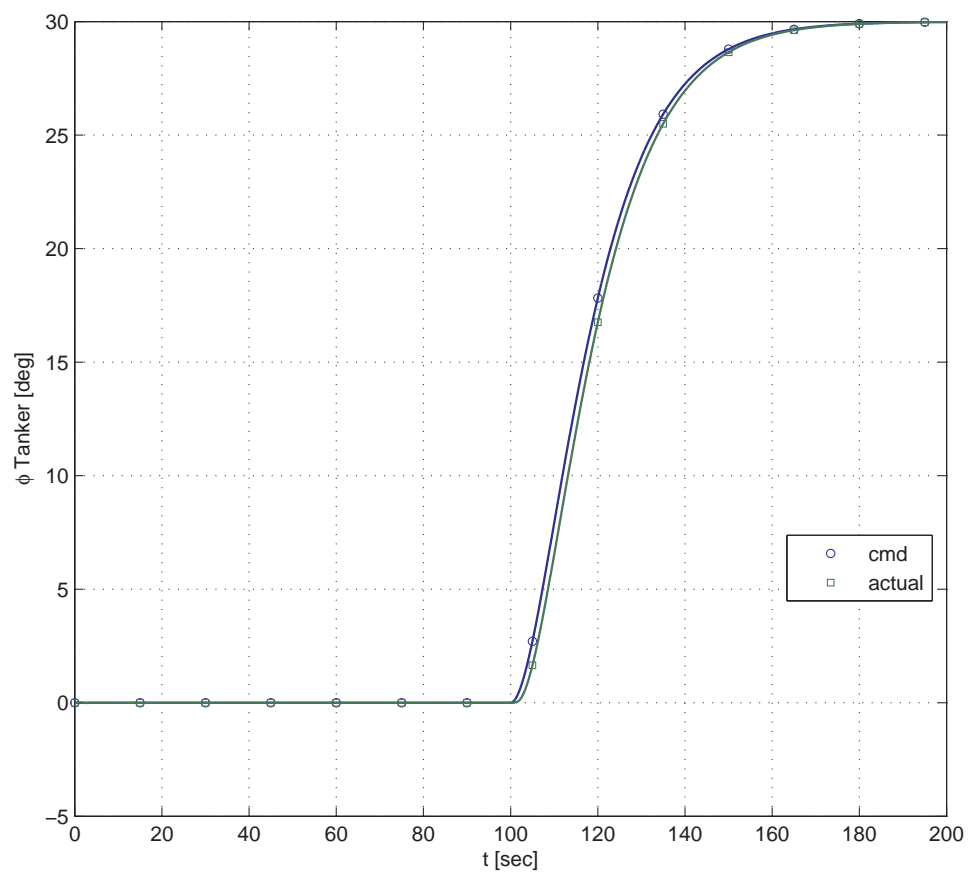

Figure 5.10. Nonlinear Simulation Tanker Performing a 30 Degree Bank. 
The simulation is initialized at a trim equilibrium point in the absence of the tanker wake-vortex induced wind disturbance on the receiver. Upon execution, the effects are brought into the simulation over a small time duration (all wind components are gradually turned on through a first order filter with a time constant of 1 second starting at 1 second in the simulation execution time). The activation of these disturbances yields an obvious transient response on the simulated receiver dynamics and is regarded unrealistic. Therefore, the simulation is allowed sufficient execution time in order for the transients to subside before the tanker begins the turning maneuver.

Simulation results of the existing LQR MIMO $[1,9,10]$ controller derived with a unity performance index are observed in Fig. (5.11) and Fig. (5.12). As anticipated, the receiver system is divergent and cannot hold the refueling position at the onset of the run, considered partly due to thrust, elevator, aileron, and rudder limitations. However, the divergence is encountered early in the time history, well before the tanker maneuver begins at 100 seconds. Therefore, the receiver response is triggered by the initial transient encountered by the tanker wake-vortex induced wind activation. Also, note that thrust vectoring is disabled for the specific receiver vehicle utilized in this study, and therefore, figures do not include $\delta_{y}$ and $\delta_{z}$ effectors. (Regardless, control saturation is still encountered when including thrust vectoring as allowed in a unity weighted performance index. For the other two performance indices to be analyzed in the nonlinear model, the $\mathbf{R}$ control action penalty matrix is set to minimize use of thrust vectoring in the Kalman gain solution). The Friedland compensation also quickly saturates control power with an ideal performance index as observed in Fig. (5.13) and Fig. (5.14), leading to a divergent station-keeping response.

Beyond the failed tracking task, both closed loop systems (baseline and new Friedland compensation) lead to the loss of controlled flight indicated by the erratic responses in airspeed $(V)$, angle of attack $(\alpha)$, and angle of sideslip $(\beta)$ with a unity performance 
index. Additionally, Euler angles (not depicted) oscillate throughout the time histories indicating the receiver tumbles in attitude after the instability is encountered. Clearly, a unity weighted performance index solution for either linear quadratic regulator control law is not a viable option. However, the remaining question is whether (i) the control action required exceeds the available position and rate limits of the effectors due to weights that are too low in the penalty matrix, $\mathbf{R}$, or (ii) the controller simply failed to stabilize the system which eventually results in effector saturation. The latter item (ii) may be caused if the linear model used to generate the controller is invalid and poorly represents the nonlinear system, or if the nominal conditions utilized in the gain scheduling fail to encompass the operating regime. This question is further discussed in Chapter 6 and recommended as a future work investigation. 

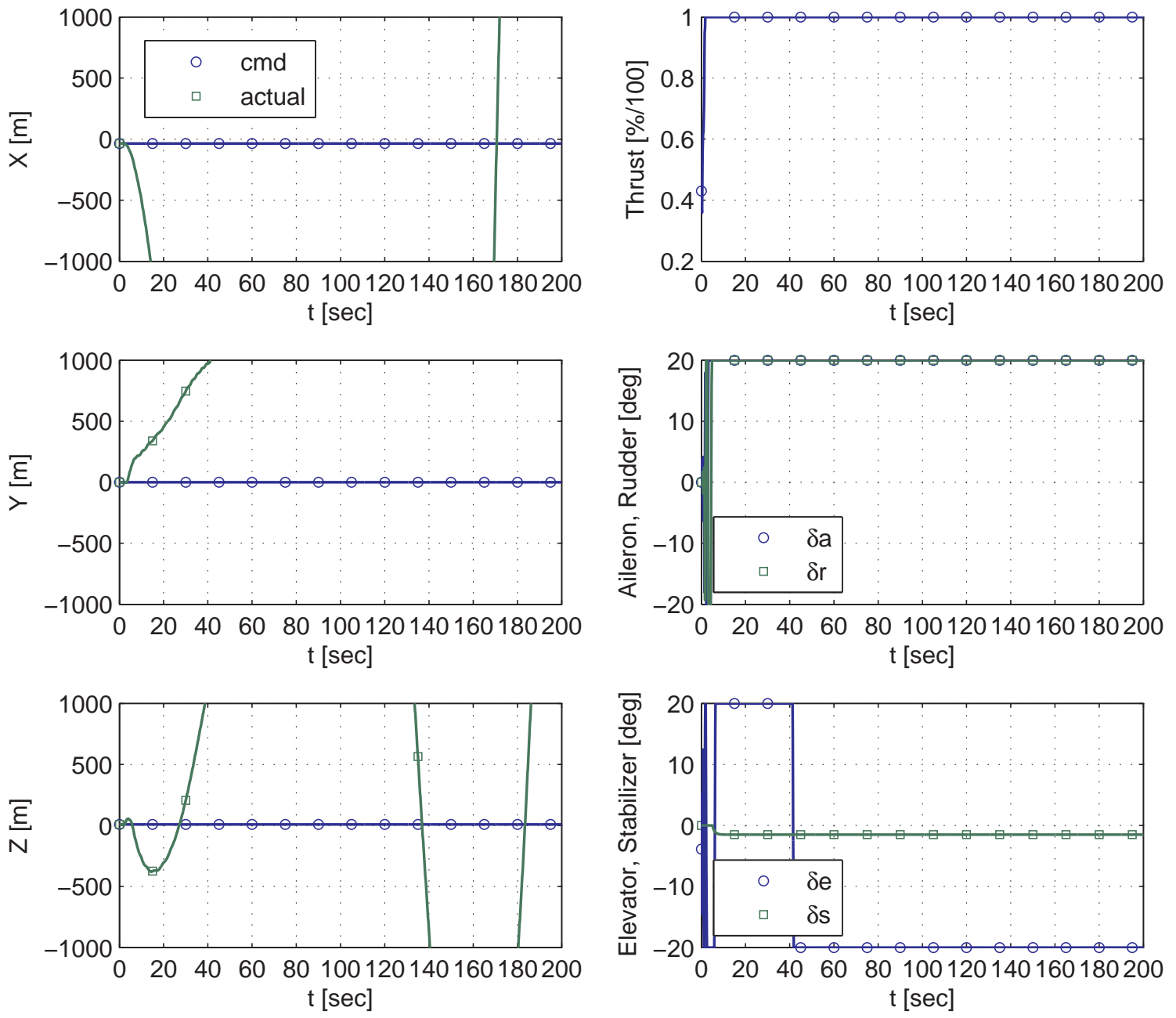

Figure 5.11. Unity Weight Q, R: Station-Keeping and Effectors for Nonlinear Simulation (Interactive Flow Field Only) LQR MIMO Control while Tanker Performs a 30 Degree Bank. 

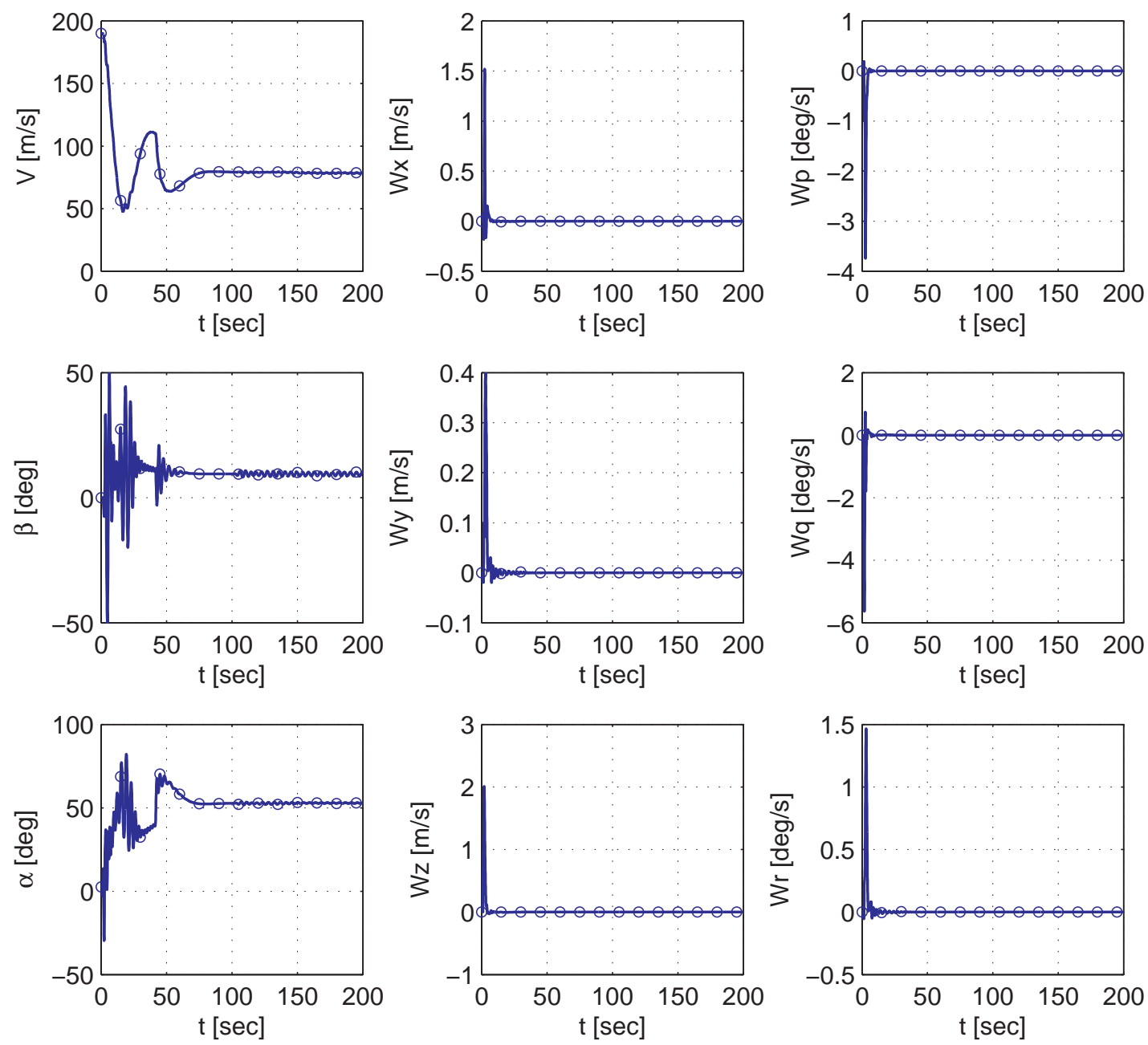

Figure 5.12. Unity Weight Q, R: $V, \beta, \alpha$, and Winds for Nonlinear Simulation (Interactive Flow Field Only) LQR MIMO Control while Tanker Performs a 30 Degree Bank. 

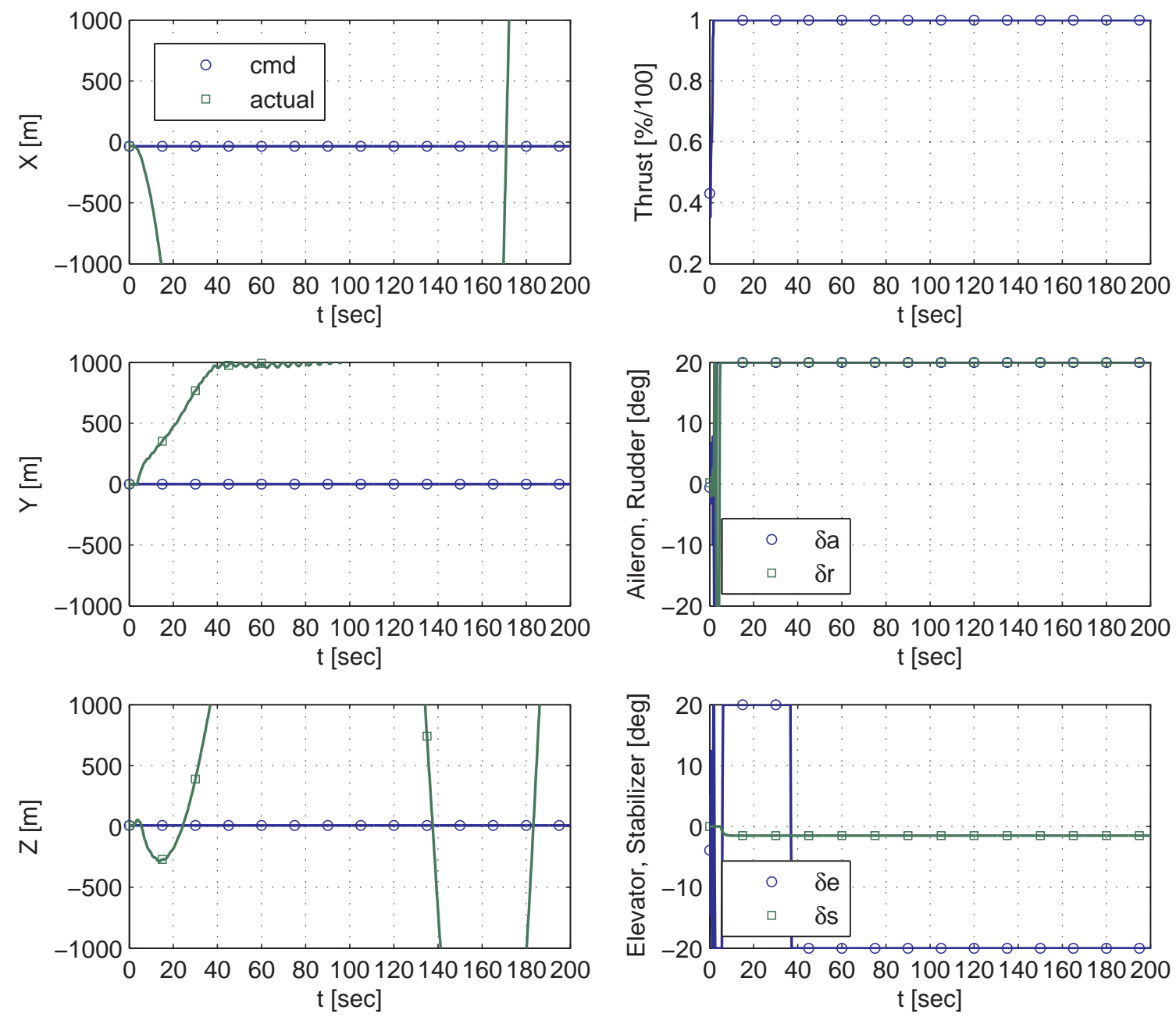

Figure 5.13. Unity Weight Q, R: Station-Keeping and Effectors for Nonlinear Simulation (Interactive Flow Field Only) LQR MIMO + Friedland Control Disturbance Rejection while Tanker Performs a 30 Degree Bank. 

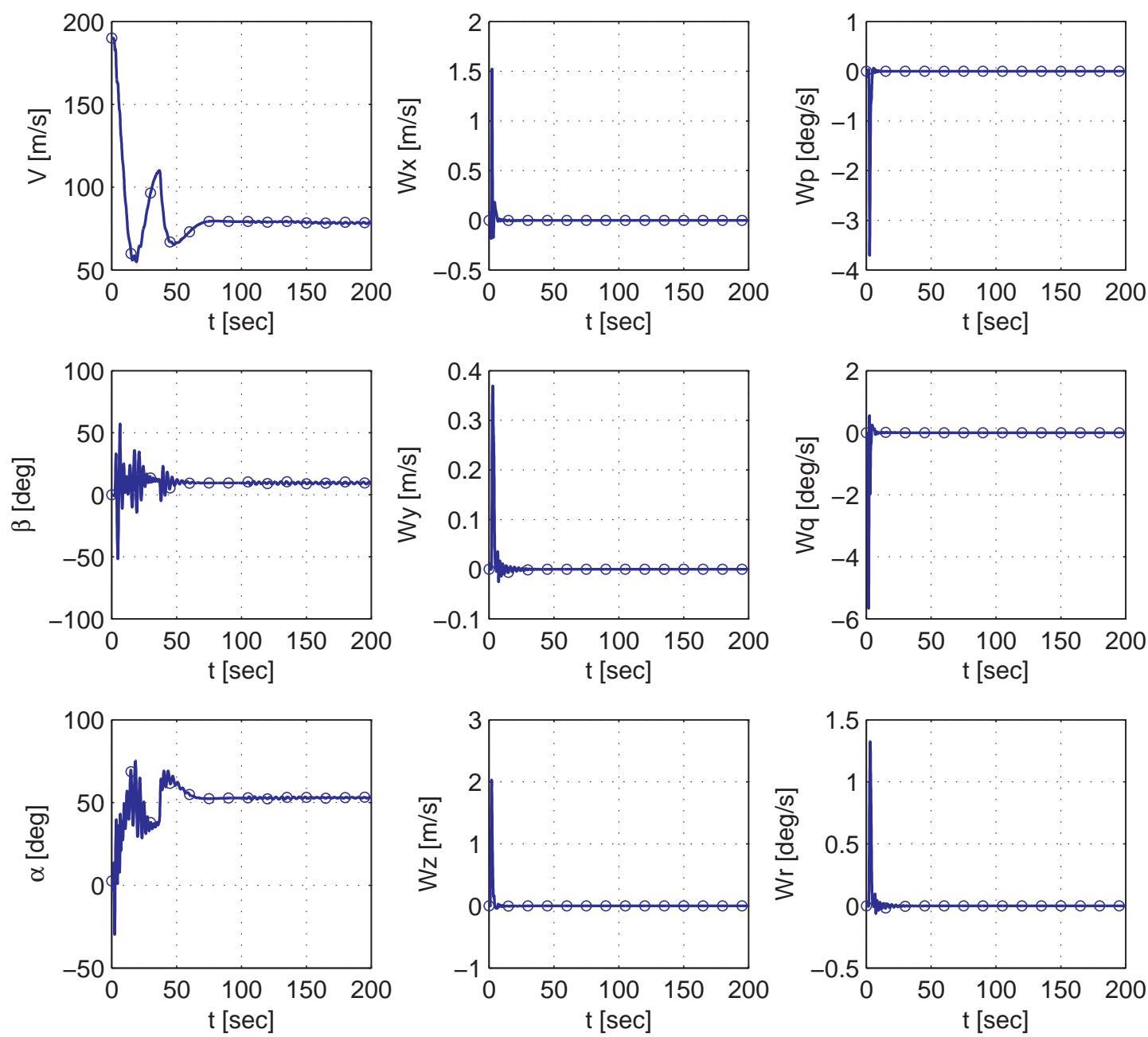

Figure 5.14. Unity Weight Q, R: $V, \beta, \alpha$, and Winds for Nonlinear Simulation (Interactive Flow Field Only) LQR MIMO + Friedland Control Disturbance Rejection while Tanker Performs a 30 Degree Bank. 
Fig. (5.15) and Fig. (5.16) repeats the simulation with the existing LQR MIMO $[1,9,10]$ controller and performance index. Poor tracking performance is observed with nearly a $25 \mathrm{~m}(82 \mathrm{ft})$ lateral deviation and a transient duration of 50 seconds. Interestingly, the controller eventually recovers and the steady state error observed in the linear analysis (nominal conditions three through six in Fig. (5.5)) is not apparent. (Note the wind components presented in Fig. (5.16) describe the tanker flow field resolved into receiver body axis components as effective wash and vorticity freestream disturbance components). Fig. (5.17) and Fig. (5.18) on the other hand, demonstrate improvement by solely enabling the Friedland compensation without modifying the existing performance index. Lateral deviation is reduced from $25 \mathrm{~m}(82 \mathrm{ft})$ to approximately $7 \mathrm{~m}(23$ $\mathrm{ft})$, although there is a $1 \mathrm{~m}(3.28 \mathrm{ft})$ overshoot upon controller recovery. Additionally, the transitory response duration appears roughly equivalent to the baseline controller at 50 seconds with the baseline performance index gain set driving the new Friedland control laws. Fig. (5.19) presents a direct comparison of the two controllers with phase plane plots for the time segment of interest from 95 seconds to 200 seconds (for evaluation of the receiver station-keeping response when the tanker initiates the turn at 100 seconds). In each phase plane, the desired station-keeping performance is indicated with a tracking box centered about the relative position command. The size of the tracking box allows for a half of meter of deviation from the contact position on all axes and therefore represents a three dimensional cubic meter box in space relative to the tanker. The $x-z$ phase plane indicates there is a $2 \mathrm{~m}(6.5 \mathrm{ft})$ degradation in the axial $x$ direction with the new Friedland controller and baseline performance index, although the $18 \mathrm{~m}$ (60 ft) improvement in the lateral $y$ axis is considered to outweigh this aspect. Clearly, neither controller meets the desired tracking performance of maintaining position within a half a meter of contact position and is addressed ahead with a performance index revision. 

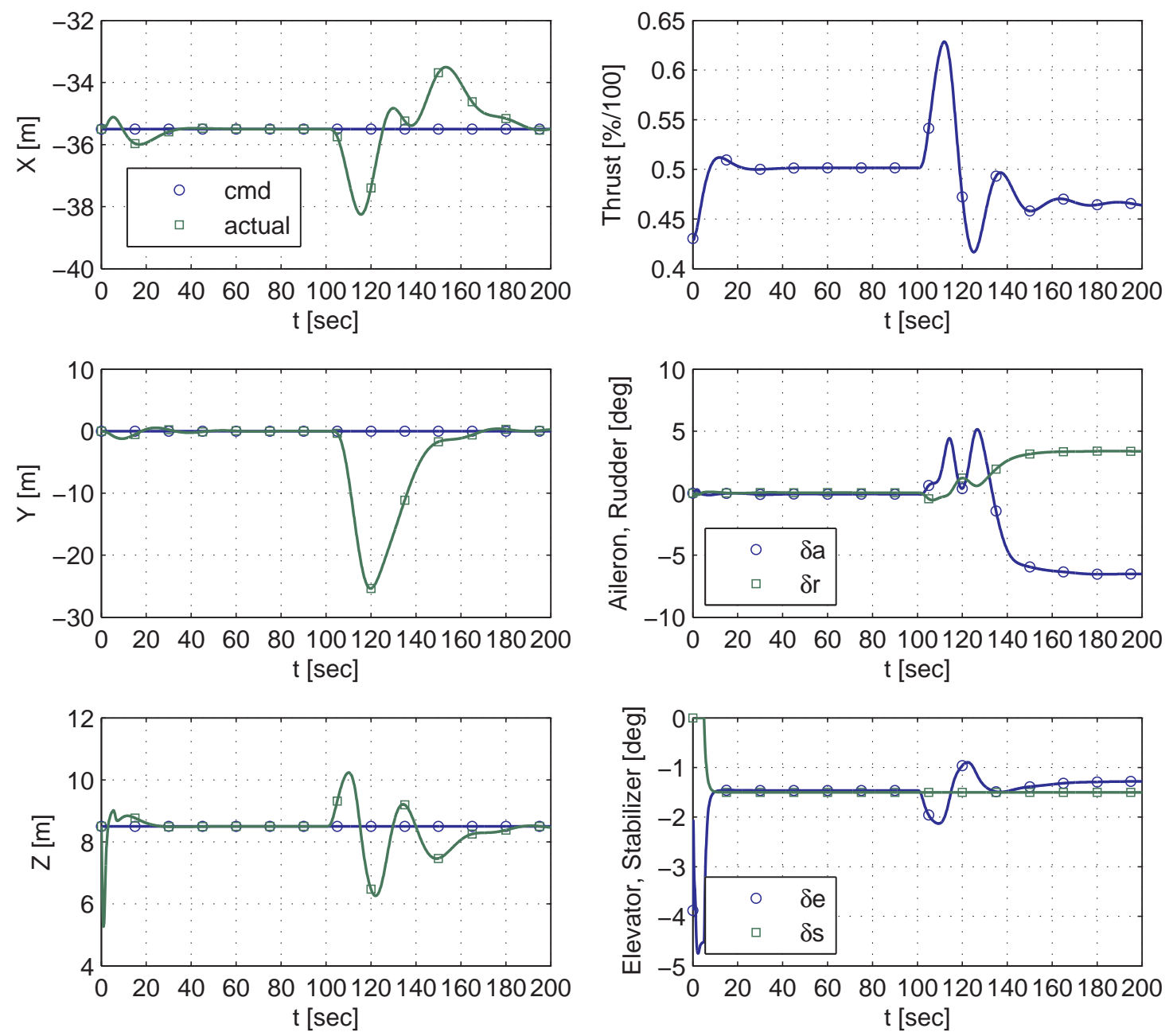

Figure 5.15. Currently Employed Q, R: Station-Keeping and Effectors for Nonlinear Simulation (Interactive Flow Field Only) LQR MIMO Control while Tanker Performs a 30 Degree Bank. 

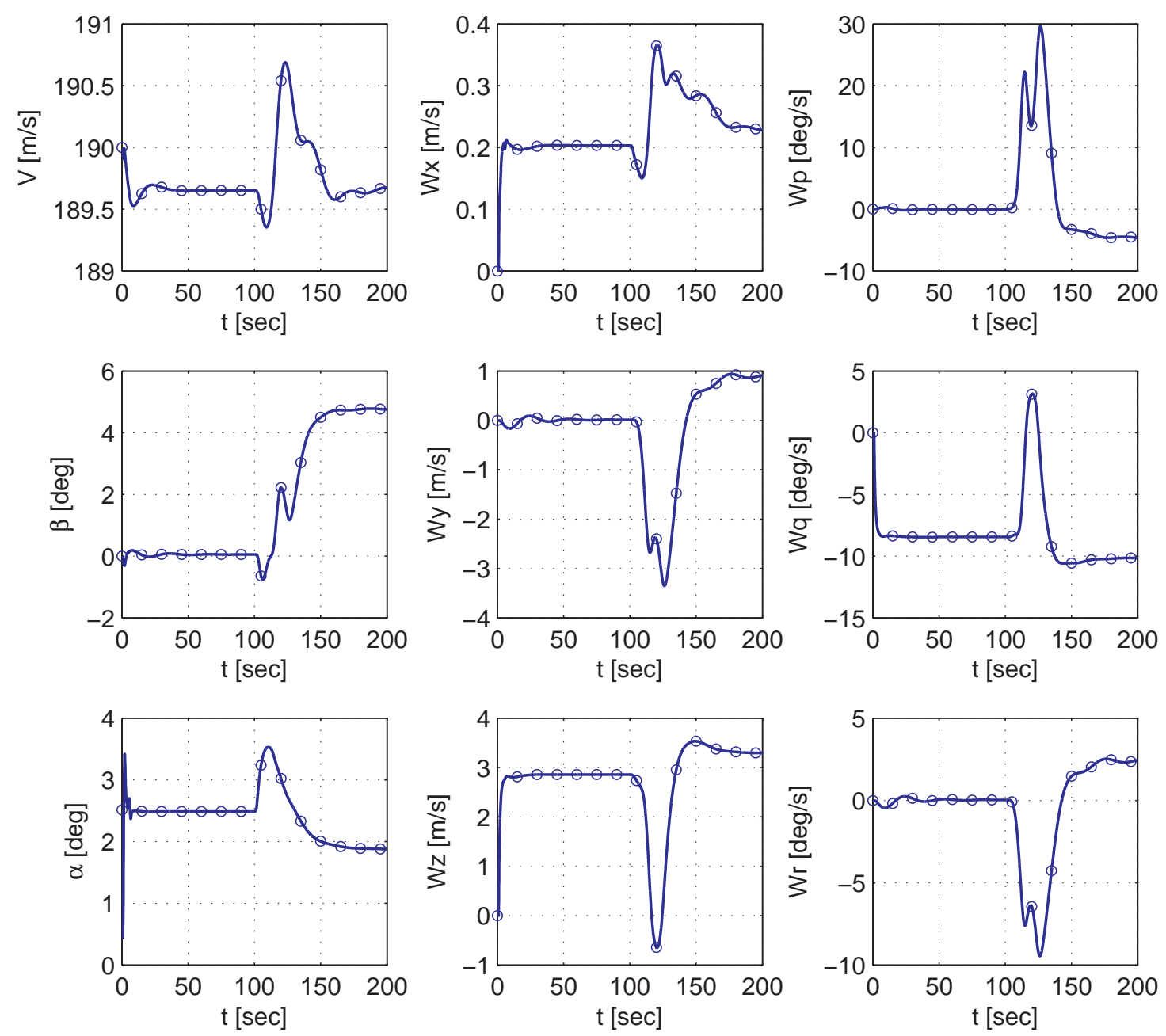

Figure 5.16. Currently Employed Q, R: $V, \beta, \alpha$, and Winds for Nonlinear Simulation (Interactive Flow Field Only) LQR MIMO Control while Tanker Performs a 30 Degree Bank. 

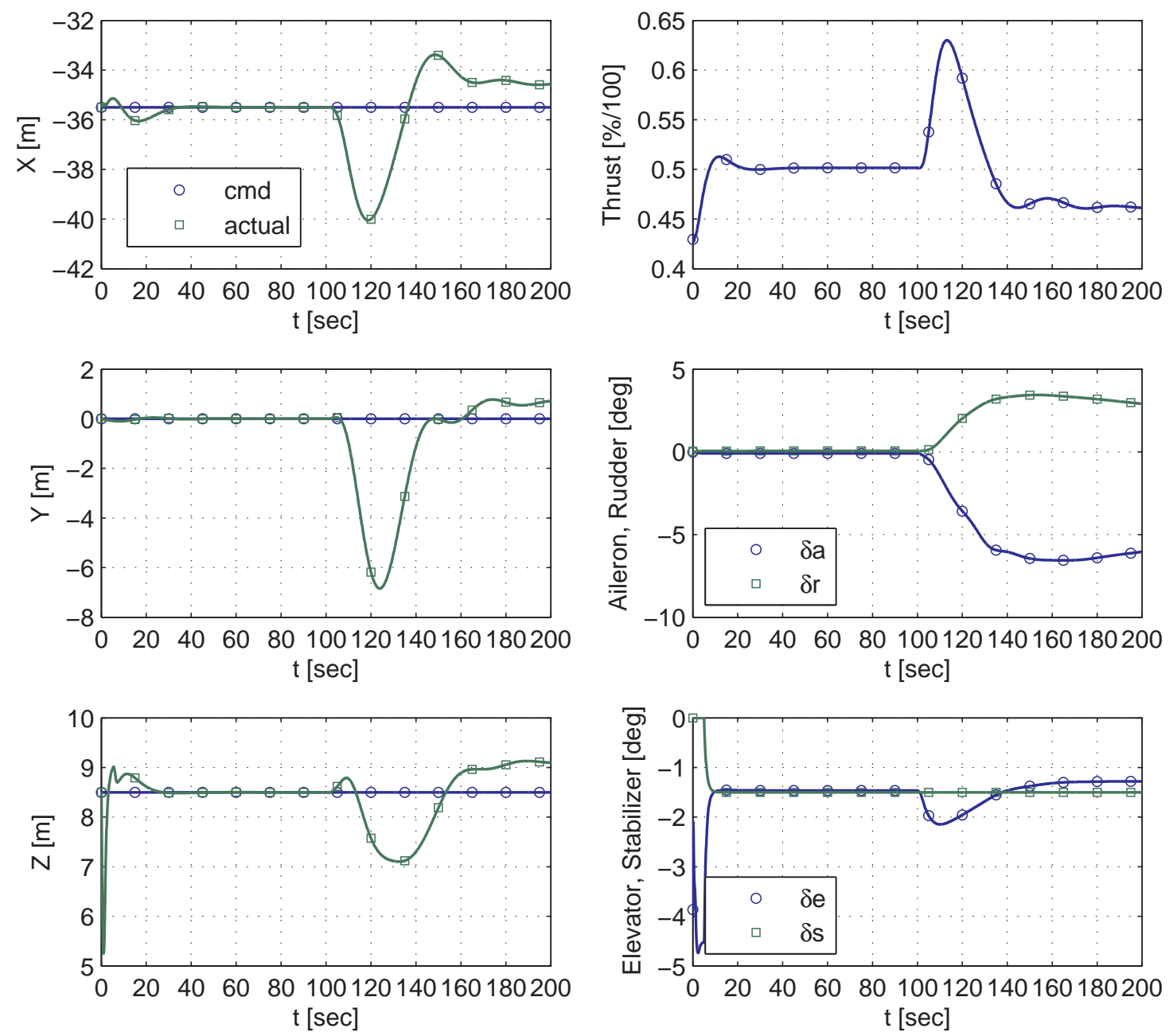

Figure 5.17. Currently Employed Q, R: Station-Keeping and Effectors for Nonlinear Simulation (Interactive Flow Field Only) LQR MIMO + Friedland Control Disturbance Rejection while Tanker Performs a 30 Degree Bank. 

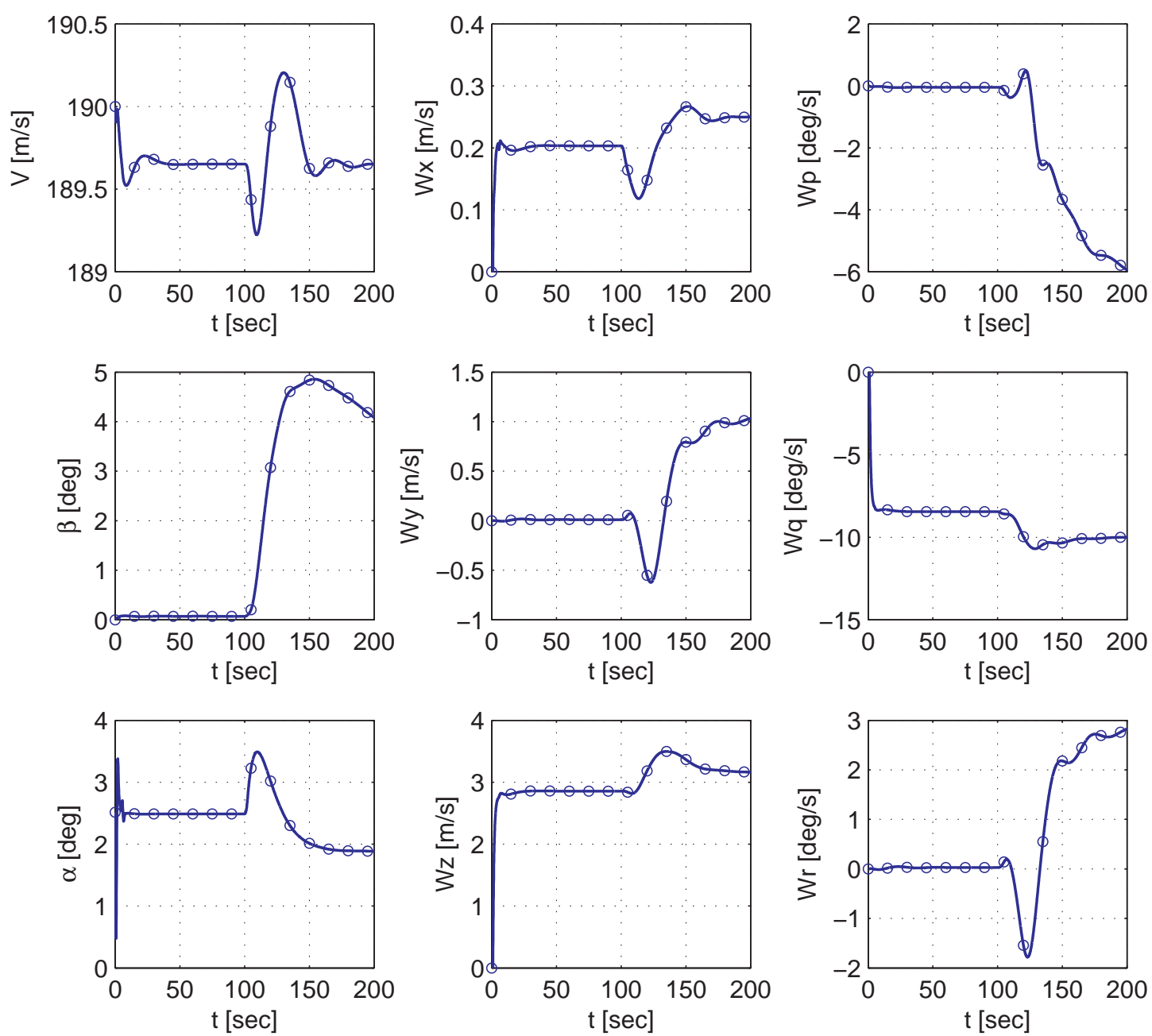

Figure 5.18. Currently Employed Q, R: $V, \beta, \alpha$, and Winds for Nonlinear Simulation (Interactive Flow Field Only) LQR MIMO + Friedland Control Disturbance Rejection while Tanker Performs a 30 Degree Bank. 

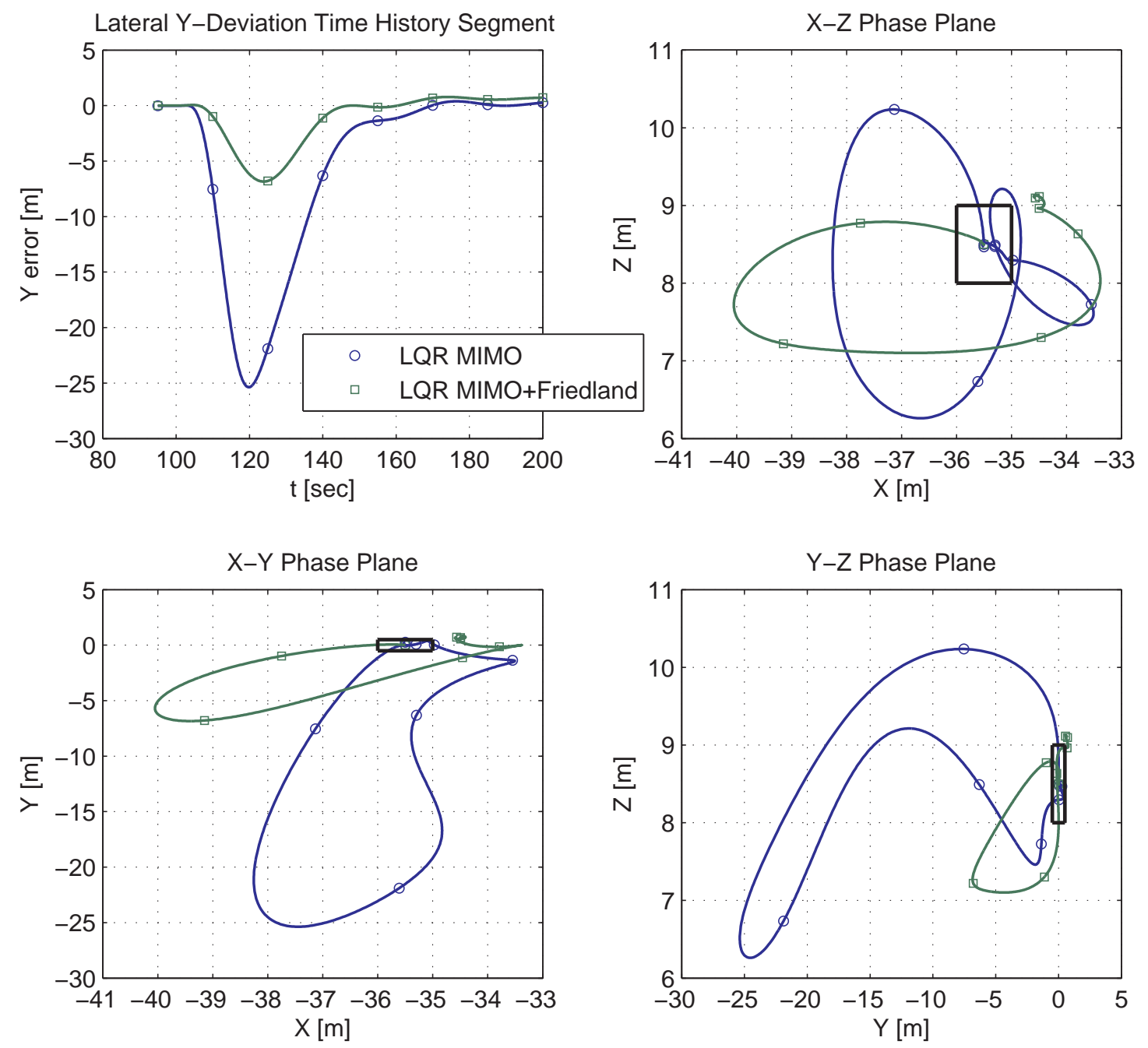

Figure 5.19. Currently Employed Q, R: Phase Plane Controller Comparison for Nonlinear Simulation (Interactive Flow Field Only) while Tanker Performs a 30 Degree Bank. 
Refer to Fig. (5.20) and Fig. (5.21) for the existing LQR MIMO [1,9,10] controller with the new revised performance index. Although the linear analysis in Fig. (5.7) indicated improved performance with the baseline controller, employment into the nonlinear model yields too costly of a control action. Effector saturation is encountered at approximately 125 seconds in the run leading to a failed task of receiver station-keeping, demonstrating the current performance index in place has been optimized to near maximum potential with the existing LQR MIMO $[1,9,10]$ infrastructure. Note the saturation appears to coincide with the tanker disturbance (right turn at 100 seconds) in this case, and the system remains stable throughout the initial simulation transient response. Nevertheless, as with the unity performance index, the receiver fails to maintain stable flight with this control law. Fig. (5.22) and Fig. (5.23), on the other hand, emphasize greater potential in performance index refinement. Enabling the Friedland disturbance rejection with the revised performance index exhibits solid tracking characteristics as the tanker flies into a 30 deg bank attitude. The new feed-forward control law, combined with $\mathbf{Q}$ and $\mathbf{R}$ revision, maintains refueling position deviation to approximately a half a meter (1.31 ft) on all axes. The settling time appears to remain approximately 60 seconds for lateral response subsidence. However, the rudder and elevator time histories in Fig. (5.23) exhibit a bifurcated response in the final 40 seconds of the run and are recommended for future investigation as a potential stability issue in Chapter 6. Regardless, the time history segment in Fig. (5.24) demonstrates the improvement potential offered by the Friedland disturbance rejection method in that desired tracking performance is maintained throughout the tanker bank maneuver [20]. Note the phase plane plots for the new revised performance index include only the new Friedland controller results as, again, the existing LQR MIMO $[1,9,10]$ controller did not successfully track contact position with the new gain set. 

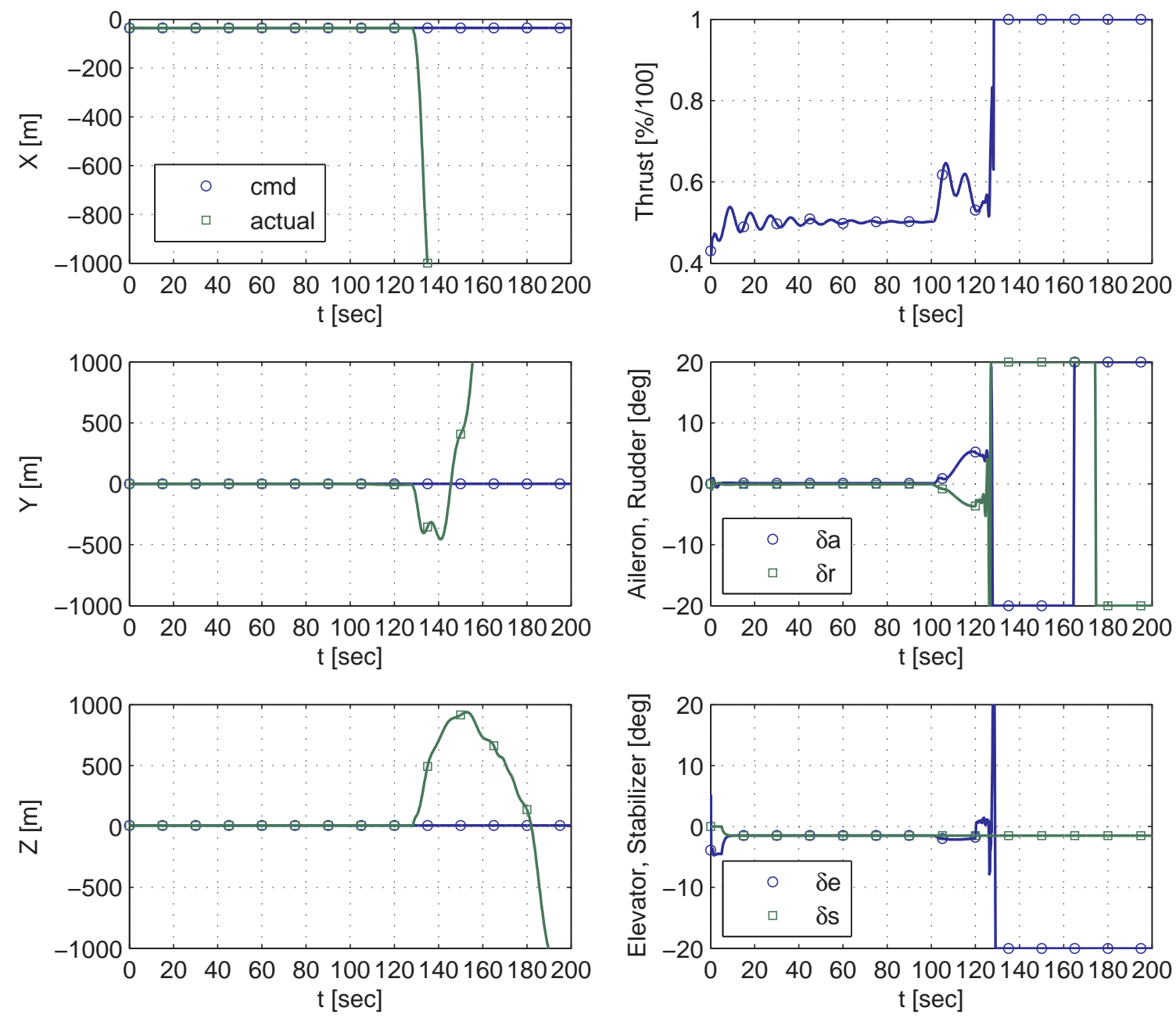

Figure 5.20. Revised Weight Q, R: Station-Keeping and Effectors for Nonlinear Simulation (Interactive Flow Field Only) LQR MIMO Control while Tanker Performs a 30 Degree Bank. 

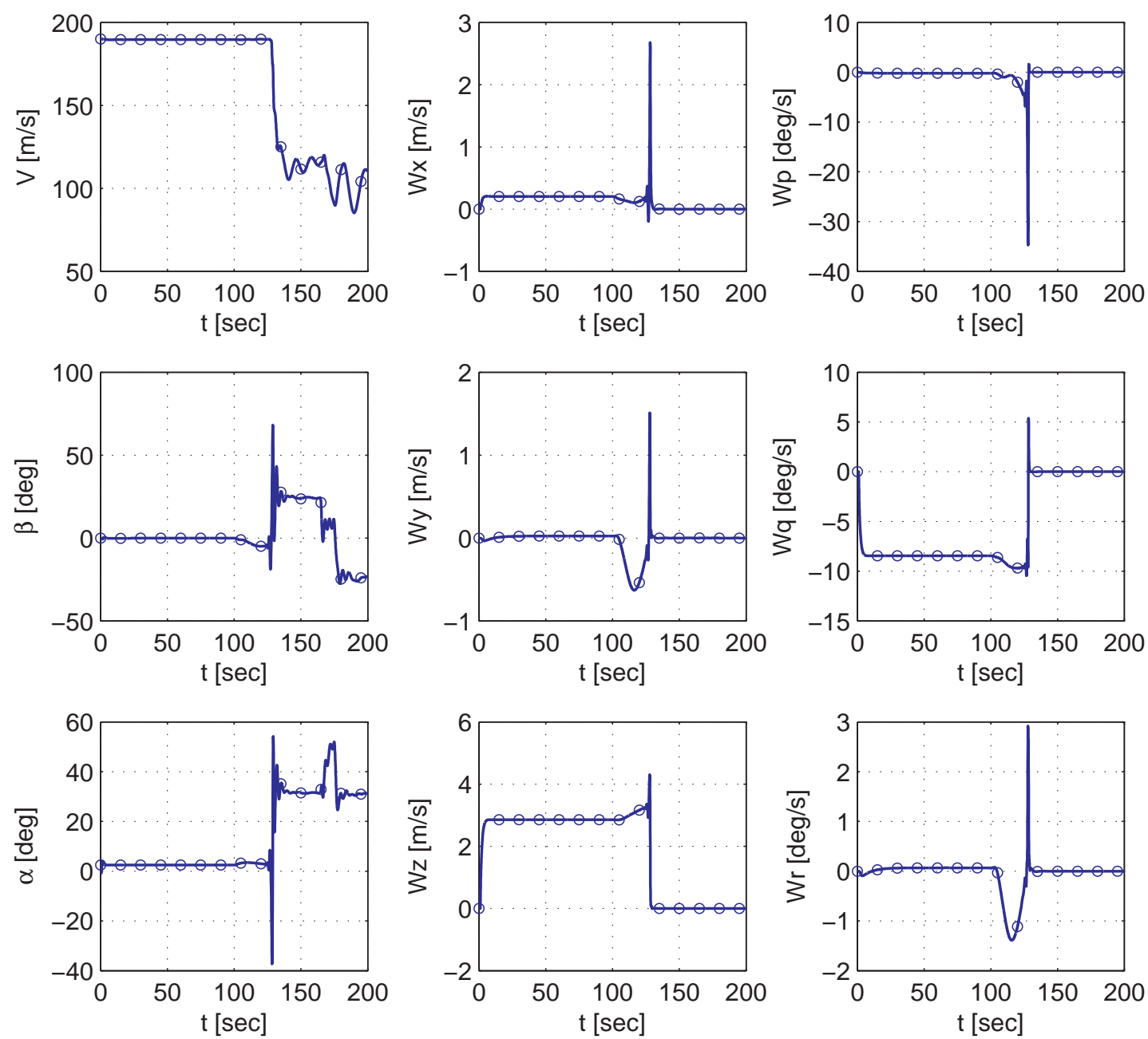

Figure 5.21. Revised Weight $\mathbf{Q}, \mathbf{R}: V, \beta, \alpha$, and Winds for Nonlinear Simulation (Interactive Flow Field Only) LQR MIMO Control while Tanker Performs a 30 Degree Bank. 

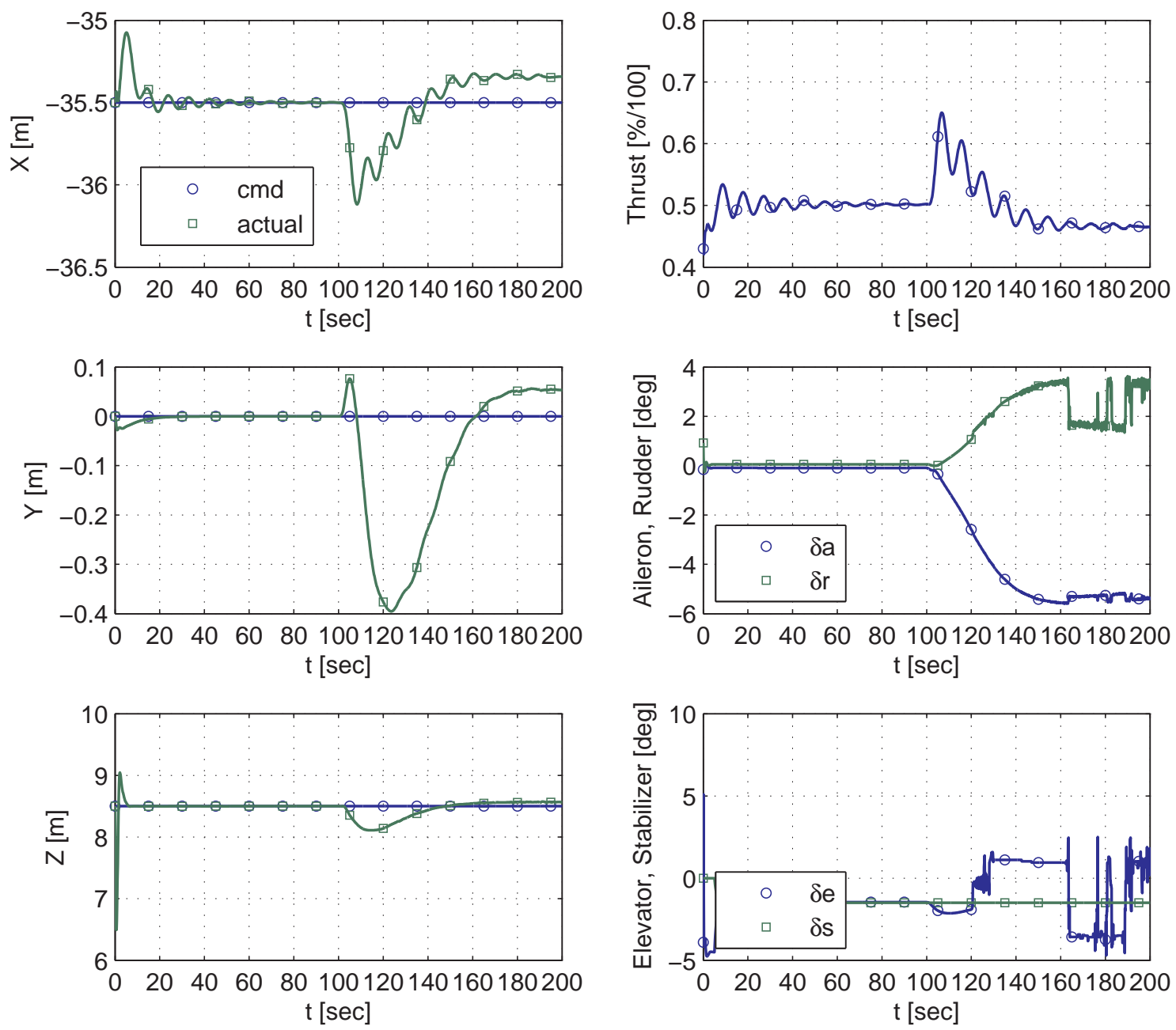

Figure 5.22. Revised Weight Q, R: Station-Keeping and Effectors for Nonlinear Simulation (Interactive Flow Field Only) LQR MIMO + Friedland Control Disturbance Rejection while Tanker Performs a 30 Degree Bank. 

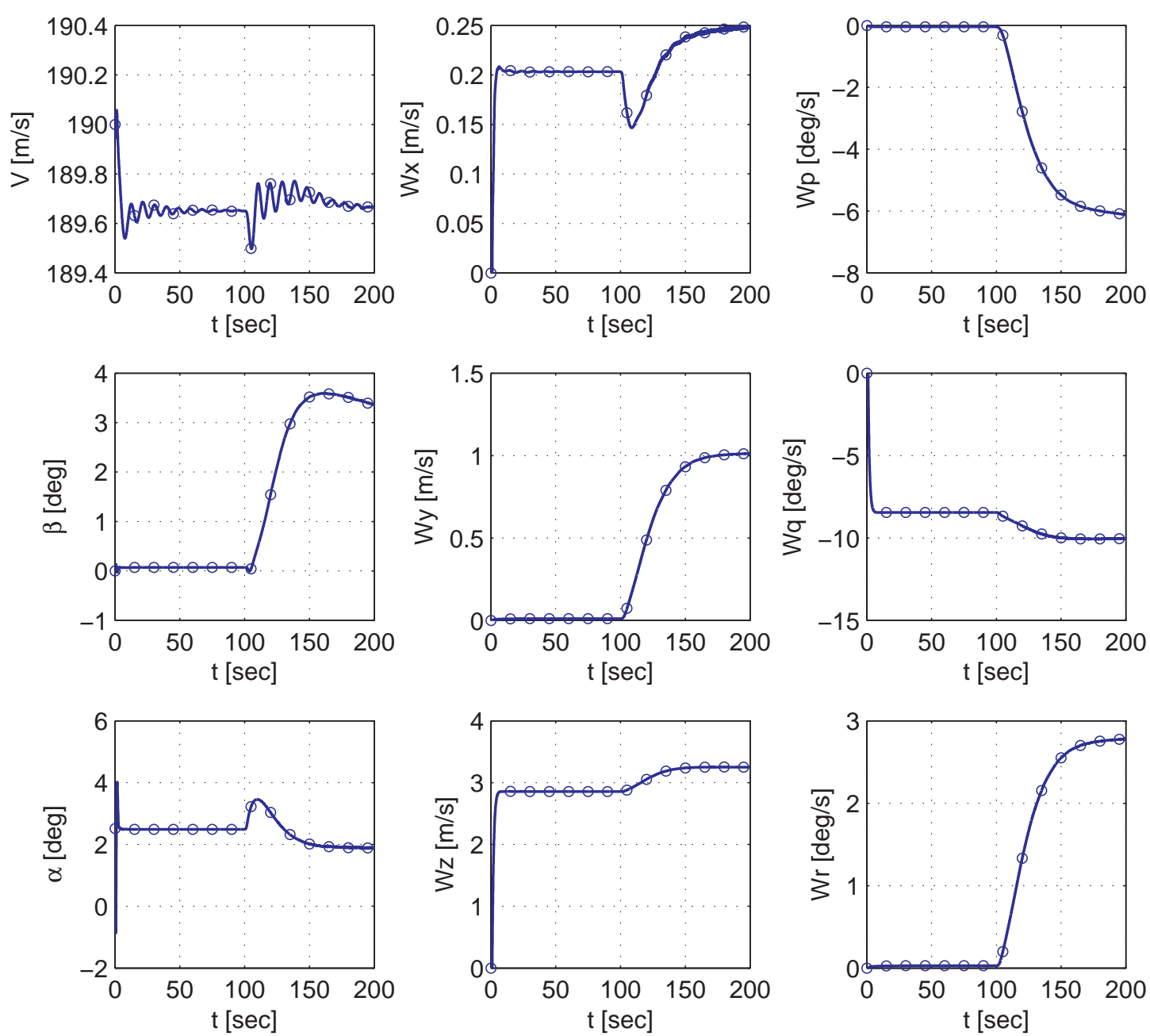

Figure 5.23. Revised Weight $\mathbf{Q}, \mathbf{R}: V, \beta, \alpha$, and Winds for Nonlinear Simulation (Interactive Flow Field Only) LQR MIMO + Friedland Control Disturbance Rejection while Tanker Performs a 30 Degree Bank. 

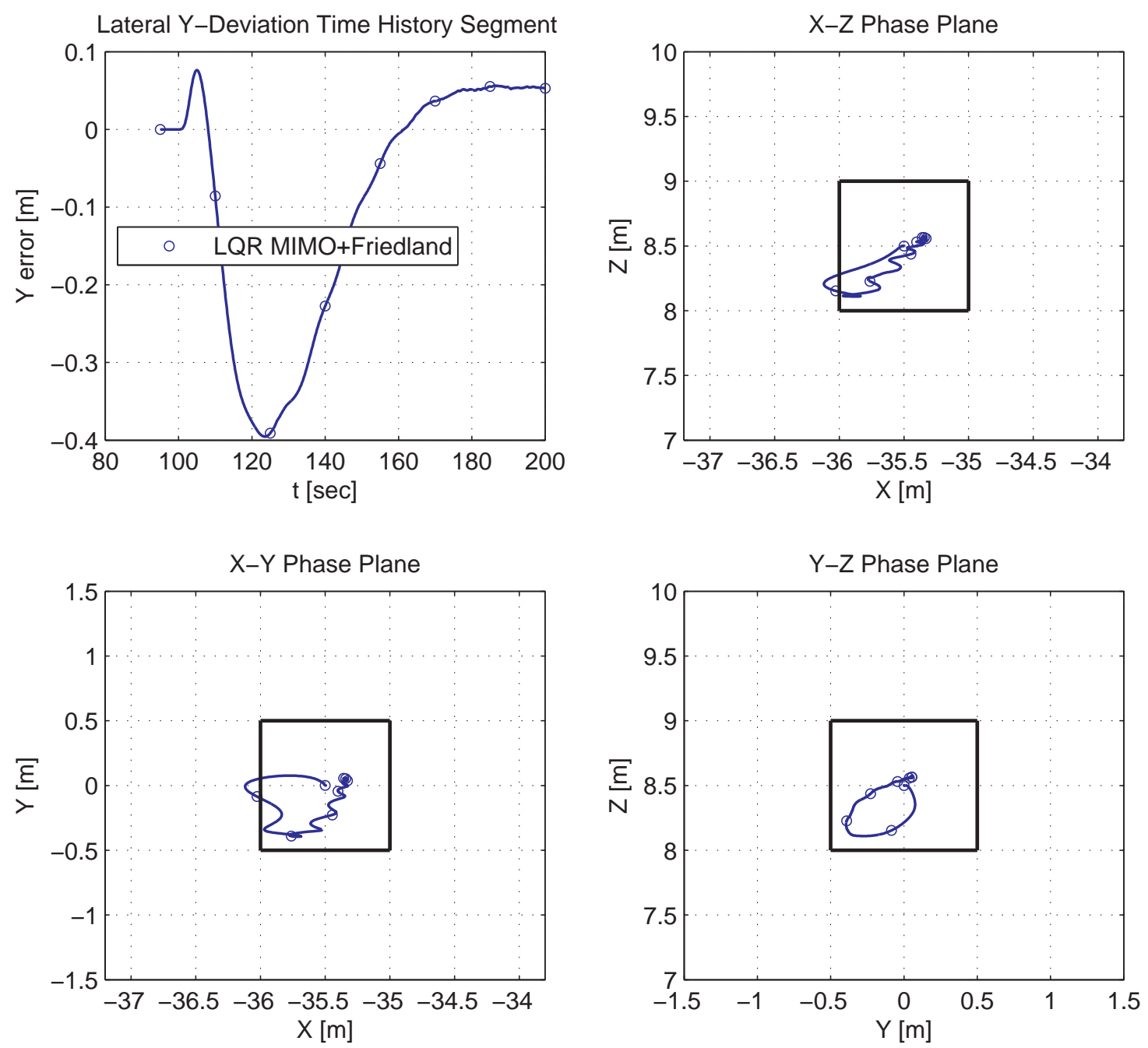

Figure 5.24. Revised Weight Q, R: Phase Plane LQR MIMO + Friedland Control Only for Nonlinear Simulation (Interactive Flow Field Only) while Tanker Performs a 30 Degree Bank. 
5.2.2 Control Law Analysis in the Presence of Tanker Flow Field and Non-Steady Prevailing Wind

The full order nonlinear simulation utilized in this study is a result of considerable efforts from prior research by Dogan et al to recreate a 2004 flight test relative position station-keeping event of a Learjet receiver tracking a KC-135 tanker [4]. The model successfully approximates the impact of the non-uniform vortex-induced tanker wind field, as presented in the time histories in the previous section [1]. The model is also able to recreate the non-steady inertial atmospheric conditions of the 2004 flight test event, denoted as prevailing winds and turbulence. With all three of these disturbances enabled, the simulation is considered to mimic actual refueling conditions, and provides the means to interrogate the robustness of the newly designed controller.

Again, similar to the evaluation procedure described in the previous section, the simulation is initialized at a trim equilibrium point in the absence of these three wind sources. Upon execution, the effects are brought into the simulation over a small time duration (all wind components are gradually turned on through a first order filter with a time constant of 1 second starting at 1 second in the simulation execution time). Finally, the tanker begins the turning maneuver at a sufficient execution time after the initial wind transient subsides on the receiver. Refer to Fig. (5.25) and Fig. (5.26) which represent the two tanker maneuvers (right and left 15 degree banked turns, respectively, at execution time 100 seconds) used for the control law analysis. (Note the tanker turn magnitude has been reduced from $30 \mathrm{deg}$ to $15 \mathrm{deg}$ in this section due to stabilization problems encountered with all wind active. Additionally, the refined performance index did not yield stable tracking for either controller in the presence of the additional disturbance and, therefore, all results in this section are for the baseline performance index only). The non-steady inertial turbulence is evident in the tracking response with tanker bank attitude deviations ranging within a degree of the bank attitude command. 


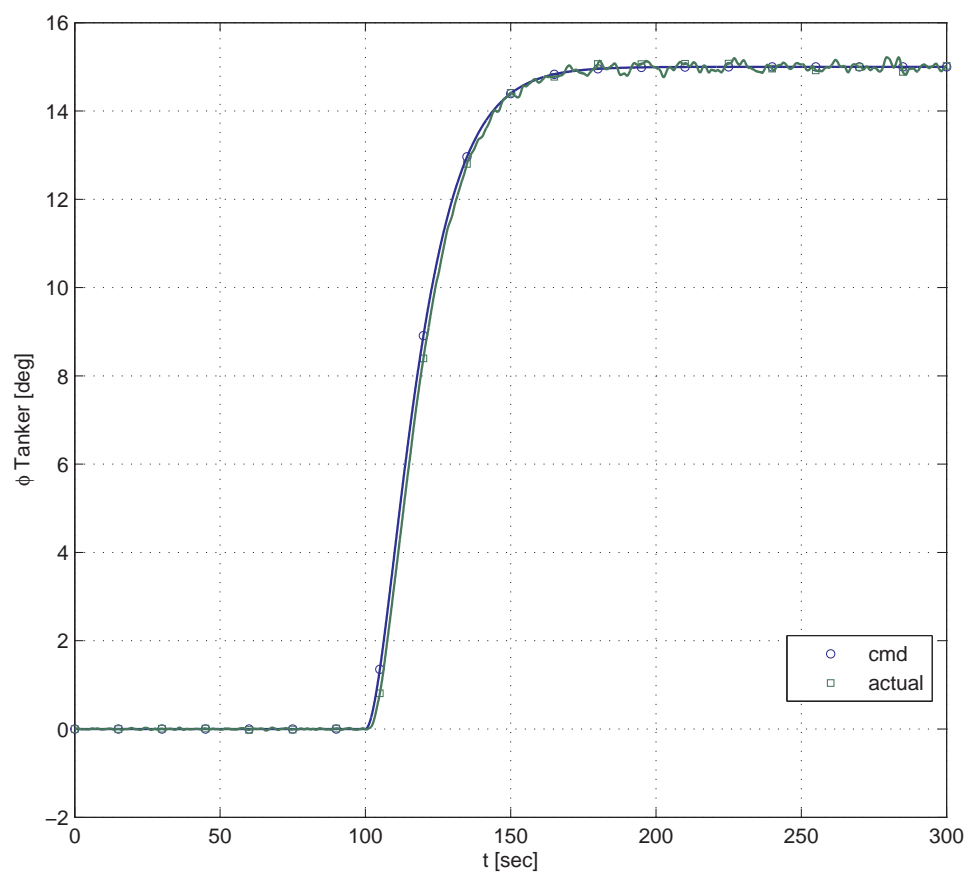

Figure 5.25. Nonlinear Simulation Tanker Performing a 15 Degree Right Bank in NonSteady Atmosphere.

Fig. (5.27) and Fig. (5.28) depict the response of the existing LQR MIMO $[1,9,10]$ controller when the tanker performs a 15 degree banked turn to the right in a realistic atmosphere with the baseline performance index gain set. Undoubtedly, the station-keeping task has become significantly more difficult with the addition of atmospheric disturbance with frequent relative position deviations from the commanded refueling box on the order of 4.5 to 5 meters (14.7 to $16.4 \mathrm{ft}$ ). Again, the initial response observed is partly considered unrealistic due to the initialization of the simulation without disturbance. However, the character (magnitude and frequency) of the deviations appear to be consistent throughout the entire time history. Also of note, the stabilizer in Fig. (5.27) does not correlate to the turbulence and maintains a steady position of approximately -1.75 deg. This control surface is selected as fixed to match the flight test event and is not an effector in the closed loop system. Future work should consider improving the linear 


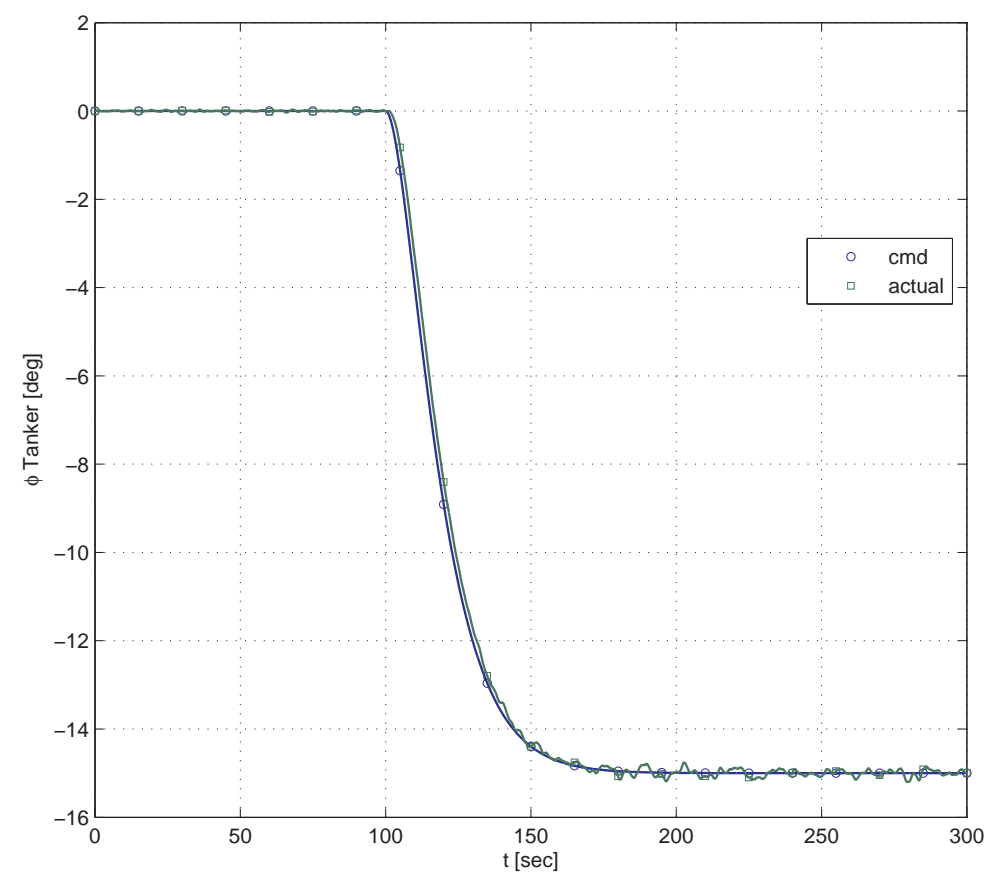

Figure 5.26. Nonlinear Simulation Tanker Performing a -15 Degree Left Bank in NonSteady Atmosphere.

model to incorporate the impact of this non-zero position and is referenced in Chapter 6.

Moving the tanker bank maneuver to the execution time at 100 seconds facilitates analysis of the effect of the accelerating target on the receiver. In other words, in Fig. (5.27), the y-lateral deviation obviously peaks as the tanker initiates the maneuver. The receiver response to maintain position on the accelerating contact position is recognizably an increase in thrust and aileron. The total velocity of the receiver is essentially constant throughout the turn as portrayed in Fig. (5.28). However, the angle of sideslip, $\beta$, increases as the receiver tracks the tanker in turn. In a manned application, pilots may consider this response as unfavorable in a conventional sense and denote the behavior as a non-coordinated level turn. Improvement of maintaining zero sideslip on the receiver is also recommended in future work Chapter 6. Also, the angle of attack, $\alpha$, response 
should be investigated as the operating range appears to increase throughout the maneuver and may be deemed unacceptable. Initial transient aside in the wind components as previously aforementioned, Fig. (5.28) time histories yield a few notable insights regarding atmospheric disturbances on the receiver. Wind components now include both inertial and tanker flow field disturbances resolved into components in the receiver body axis. At 100 seconds, the rolling and yawing vortices and the downwash on the receiver exhibit a transient response as the tanker accelerates. The forward and side washes, however, exhibit a lower frequency change considered dominated by the change in heading of the receiver exposed to prevailing winds [4]. In other words, as the receiver follows the tanker in the level turn, the body axis rotates on the inertial $x-y$ phase plane and the prevailing winds transition over time from head winds to tail winds. Likewise, the cross winds change signs throughout the turning maneuver. Finally, the pitch vortex on the receiver remains fairly constant throughout the station-keeping event and therefore is dominated by the interactive tanker flow field.

Fig. (5.29) and Fig.(5.30) present the results for repeating the case of the tanker performing a 15 degree banked turn to the right, now with the Friedland disturbance rejection [20] enabled. Immediately discernible is the absence of the peak lateral deviation near simulation time 100 seconds as encountered with the baseline controller.

Refer to Fig. (5.31) which compares the tracking errors between the two controllers in a Gaussian Probability Density Function (PDF) for the time history segment when the tanker executes the turning maneuver. Each subplot is titled with the mean $(\mu)$ and standard deviation $(\sigma)$ of the relative position error from the respective command. The PDF provides a powerful method for presenting the overall trend in the time history where the peak of the curve is centered at the mean and width of the curve is a function of the standard deviation. For instance, in Fig. (5.31), the green stripes, centered at zero deviation, indicate the goal of the desired station-keeping tracking performance (within a 
half of meter or $1.31 \mathrm{ft}$ of the command). The yellow stripes indicate a tolerable threshold of deviation (relaxed to within $1.5 \mathrm{~m}$ or $5 \mathrm{ft}$ of the command), based on performance requirements. The area under the PDF curve outside the yellow stripe equates the probability of deviation for exceeding the threshold.

Immediately noticeable in Fig. (5.31) is the significant improvement in lateral tracking with the new Friedland compensation where peak y-deviation due to the tanker turn is reduced from $12 \mathrm{~m}$ to $6 \mathrm{~m}(39.3 \mathrm{ft}$ to $19.7 \mathrm{ft})$. Also, the lateral deviation mean and standard deviation are reduced significantly with the new controller. Axial deviation for both controllers exhibits an approximate zero mean, although there is a subtle degradation apparent in x-deviation with the new control law (slightly higher standard deviation). The difference in tracking for the z-axis between the two controllers is also subtle, although the new controller offers slight improvement (decreased mean and standard deviation). Clearly, the most prominent feature in the plot resides in the lateral deviation PDF which reflects the probability of maintaining the threshold is considerably higher with the new Friedland control law.

While the new controller offers a welcomed improvement for the receiver simulation in the presence of a realistic atmosphere, the response still does not meet the desired tracking criteria established to maintain the refueling position within a half of a meter on all axes. However, this result is considered to be a limitation of the fidelity of disturbance model employed. In other words, the new disturbance rejection technique [20] successfully attenuates the exogenous system currently modeled (tanker acceleration) significantly, as proven in the previous section. In order to meet adequate tracking performance in the presence of additional disturbances (winds), the receiver linear model and controller gain set formulation should be revisited to capture these effects as recommended in Chapter 6. (Note as discussed at the conclusion of Chapter 2, refinement of the disturbance technique, with the objective of improving wind attenuation, will require two items to 
be modeled: (i) the wind impact on the transformation matrix of the exogenous state vector into the receiver state-space system (E) and (ii) the matrix governing the first order differential equation describing the wind dynamics).

An additional note regarding Fig.(5.30) is the absence of the transients observed in the vorticity rolling and yawing components with the baseline controller at the tanker maneuver time of 100 seconds. This difference is considered due to the fact the Friedland controller maintains a closer relative position to the tanker throughout the maneuver. In other words, as the receiver with the baseline controller deviates from the refueling box as the target accelerates away, the vehicle transitions to a new position within the non-uniform vortex-induced wind field aft of the tanker, effectively changing the exposure to aerodynamic coupling. On the other hand, the Friedland controller experiences less transient behavior in the non-uniform wind field due to less spatial deviation relative to the tanker. 

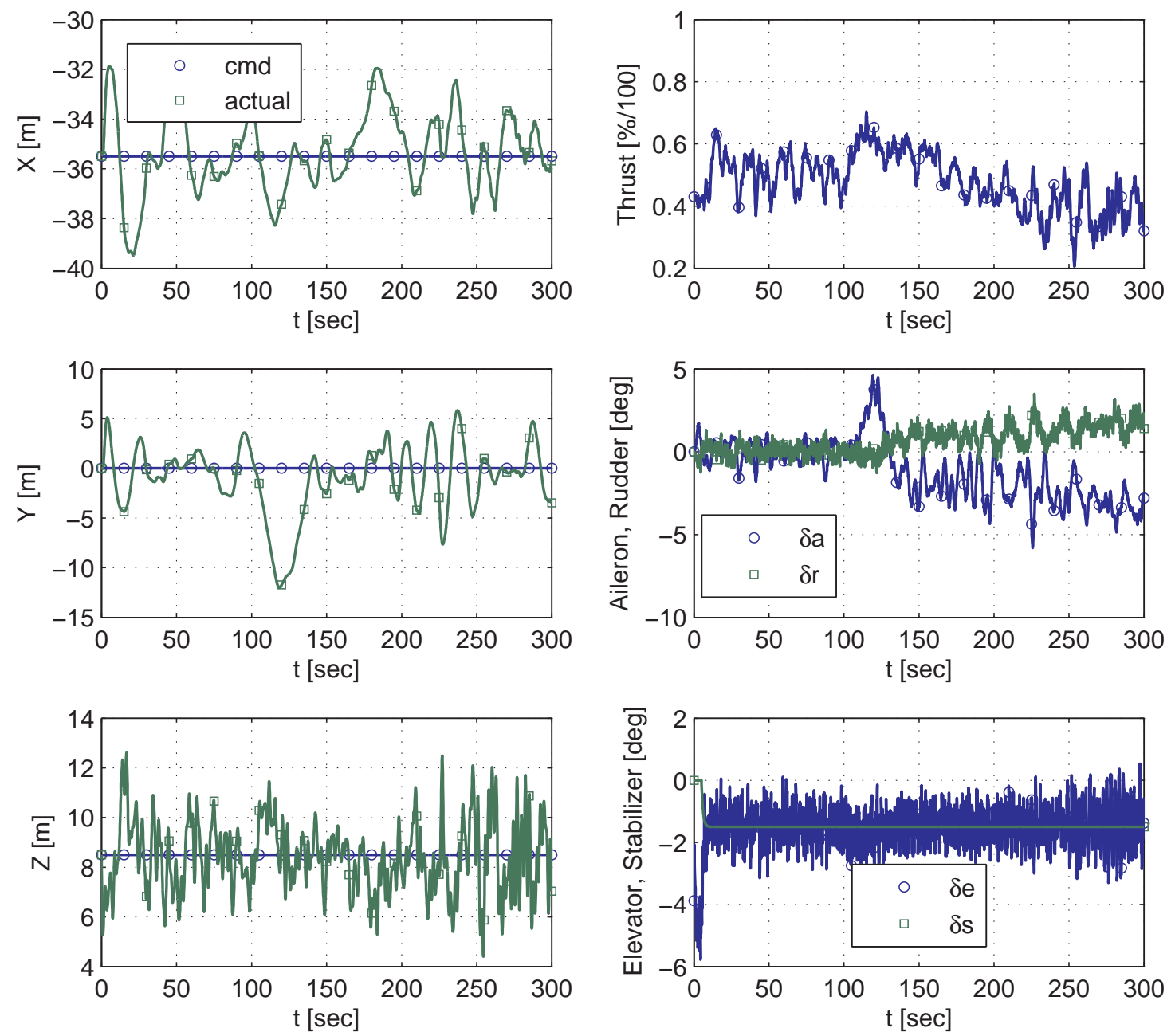

Figure 5.27. Currently Employed Q, R: Station-Keeping and Effectors for Nonlinear Simulation LQR MIMO Control while Tanker Performs a 15 Degree Right Bank in NonSteady Atmosphere. 

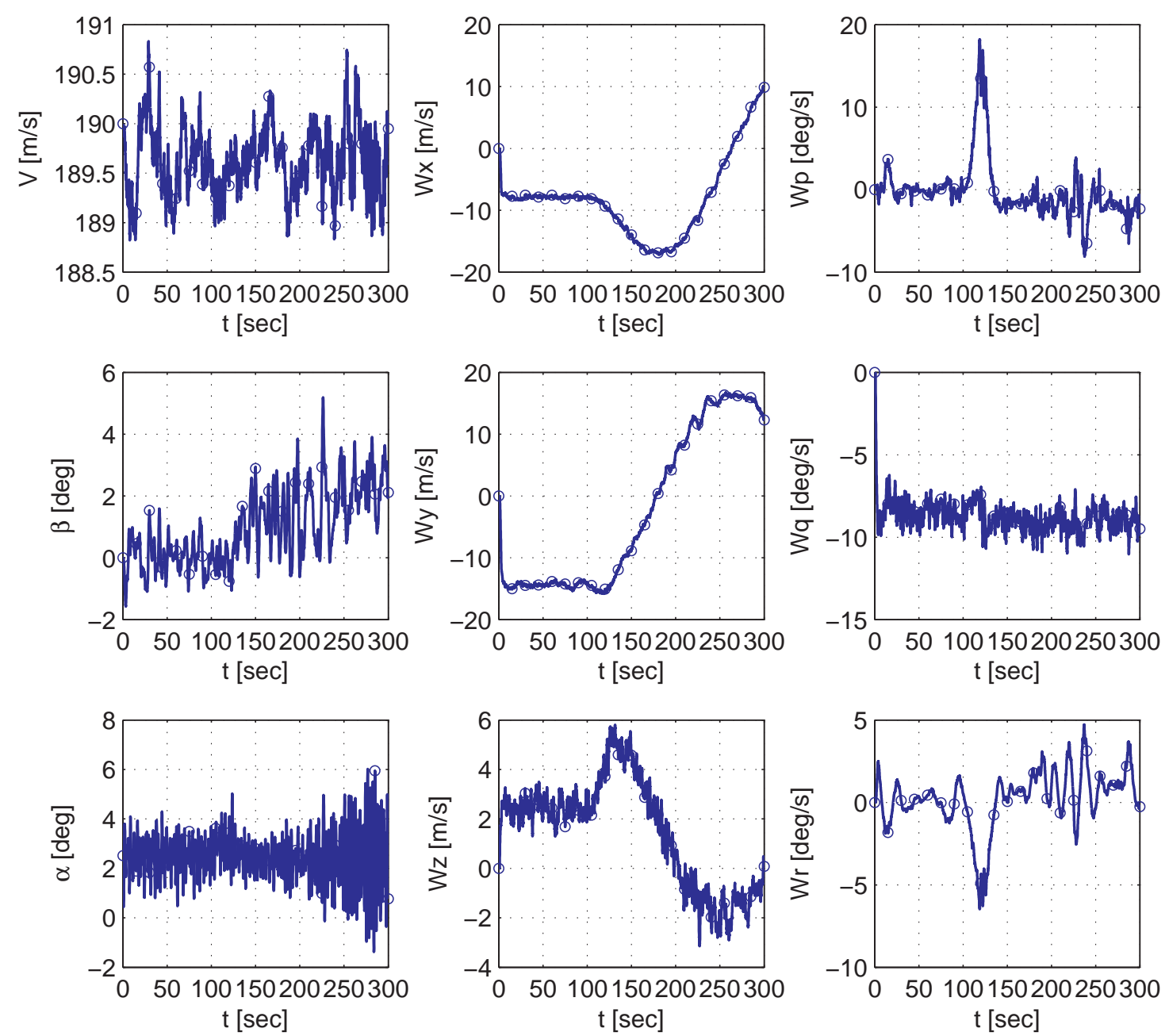

Figure 5.28. Currently Employed Q, R: $V, \beta, \alpha$, and Winds for Nonlinear Simulation LQR MIMO Control while Tanker Performs a 15 Degree Right Bank in Non-Steady Atmosphere. 

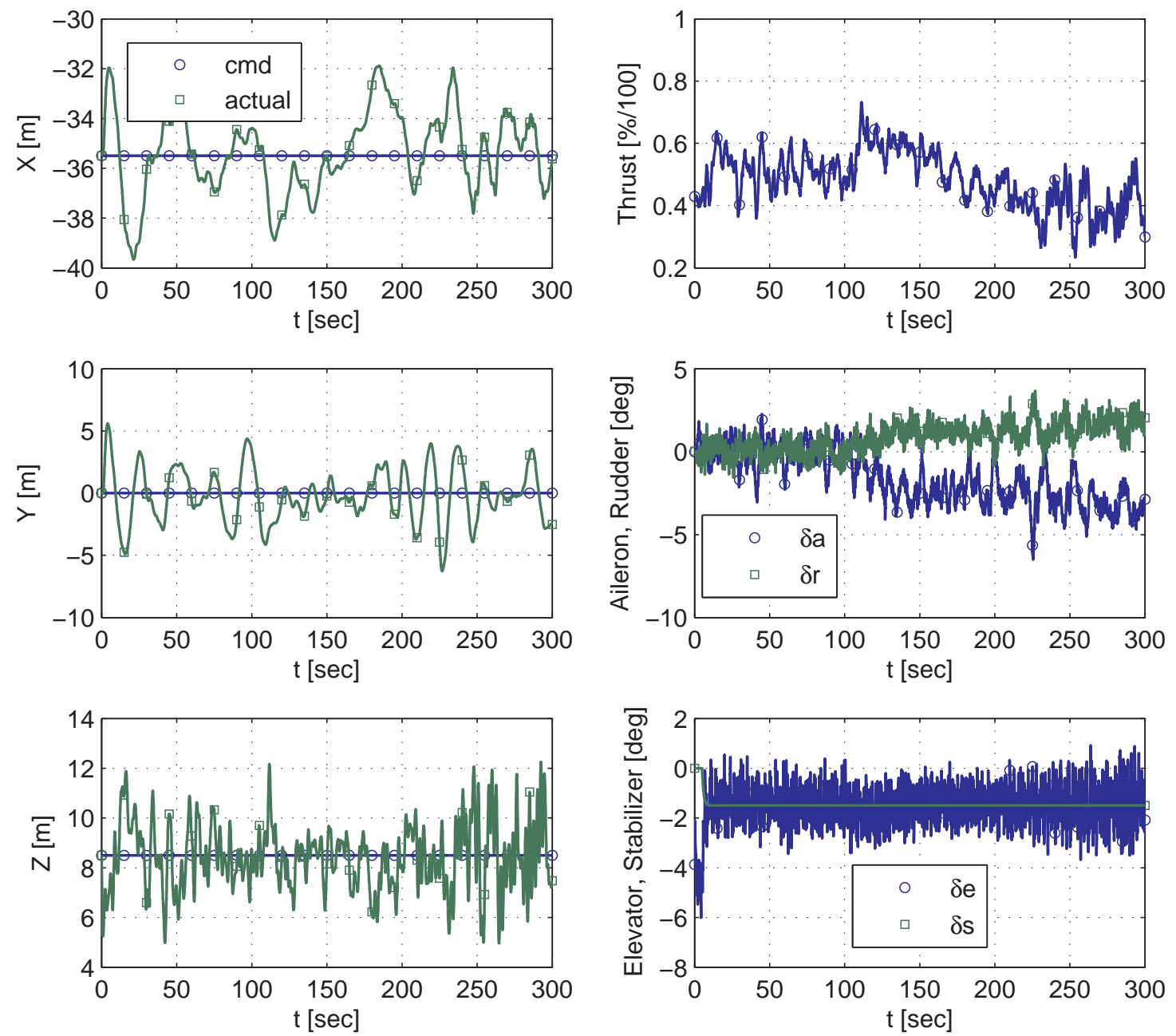

Figure 5.29. Currently Employed Q, R: Station-Keeping and Effectors for Nonlinear Simulation LQR MIMO + Friedland Control while Tanker Performs a 15 Degree Right Bank in Non-Steady Atmosphere. 

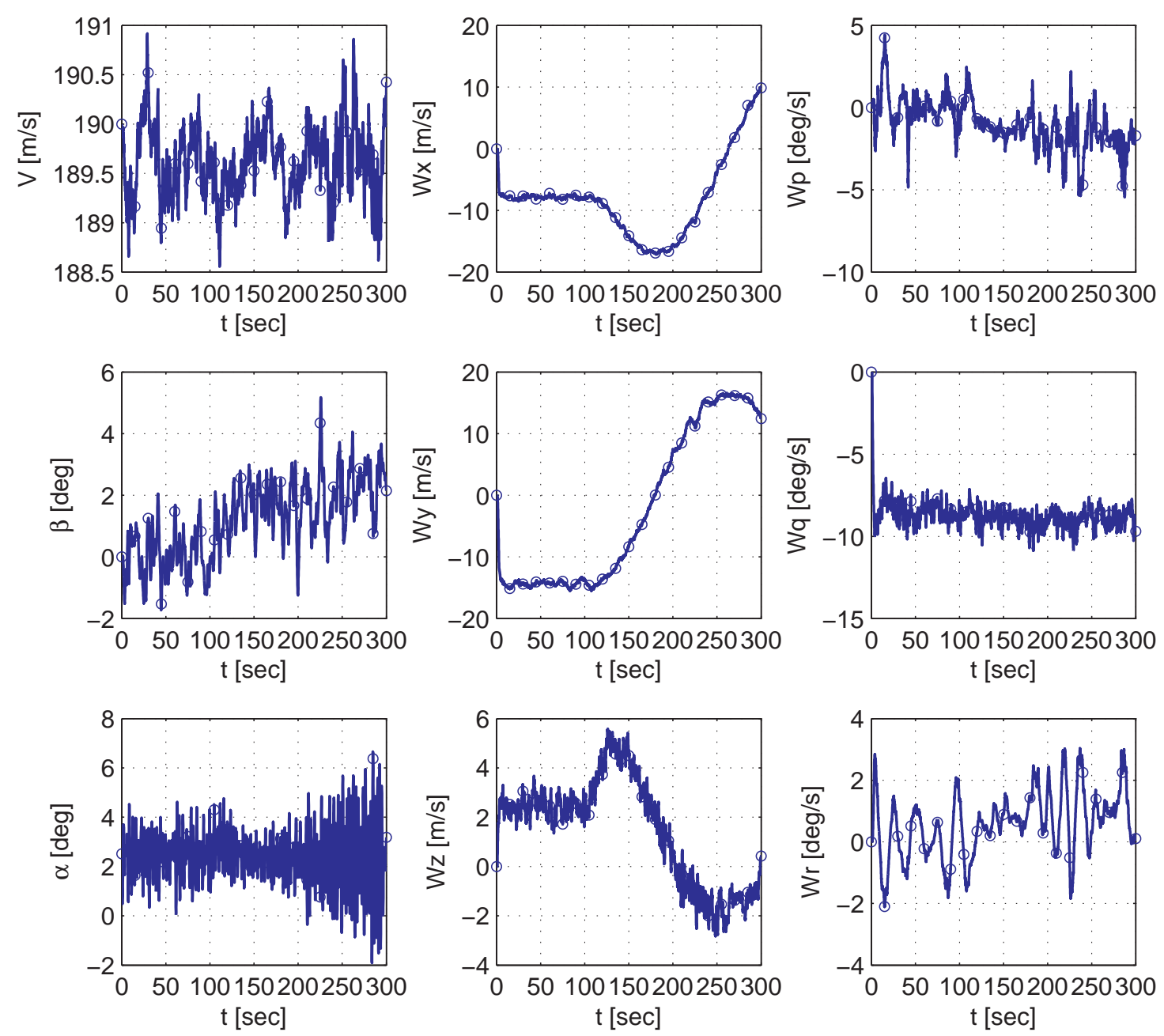

Figure 5.30. Currently Employed Q, R: $V, \beta, \alpha$, and Winds for Nonlinear Simulation LQR MIMO + Friedland Control while Tanker Performs a 15 Degree Right Bank in Non-Steady Atmosphere. 

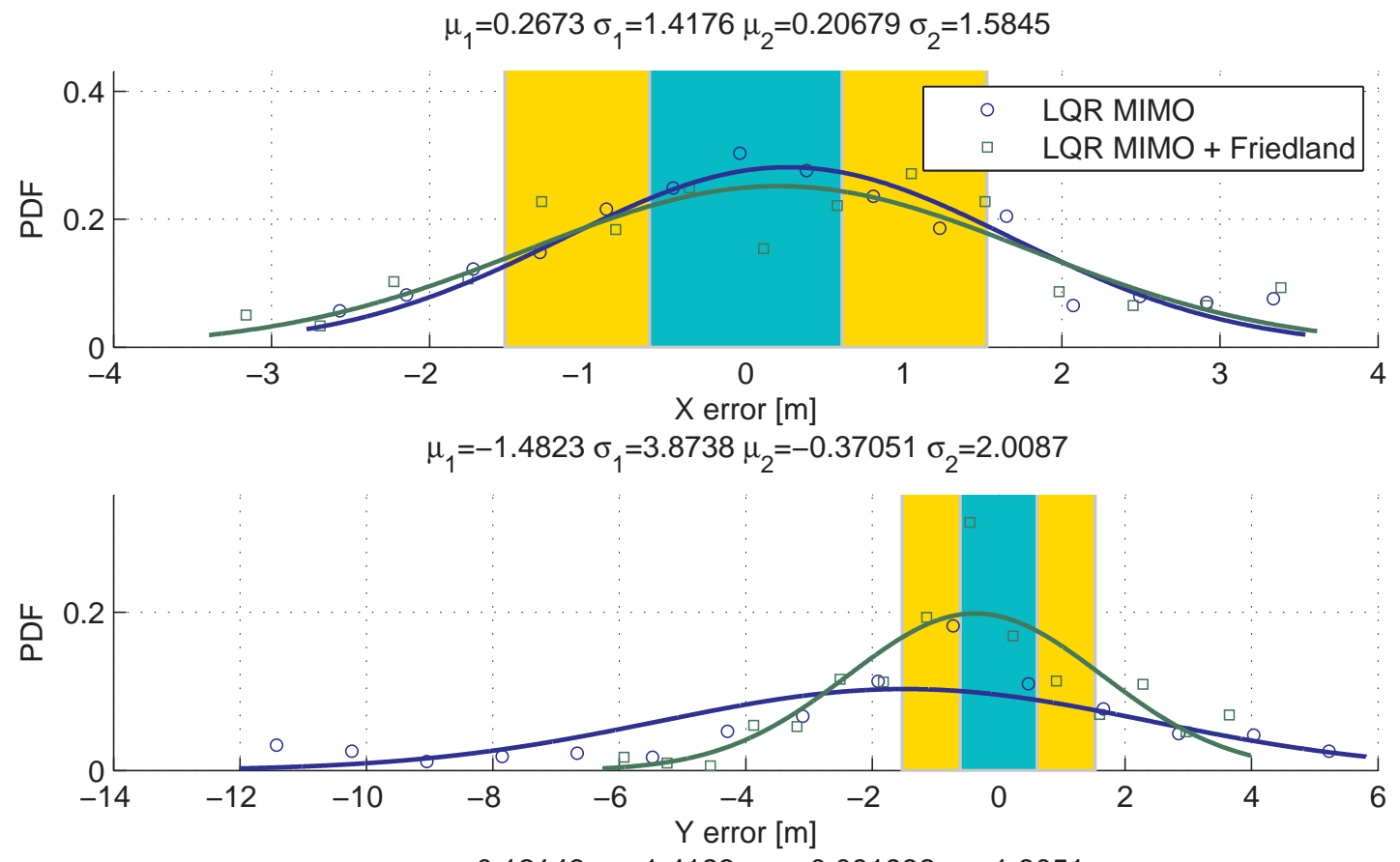

$\mu_{1}=-0.12148 \sigma_{1}=1.4123 \mu_{2}=-0.091932 \sigma_{2}=1.3051$

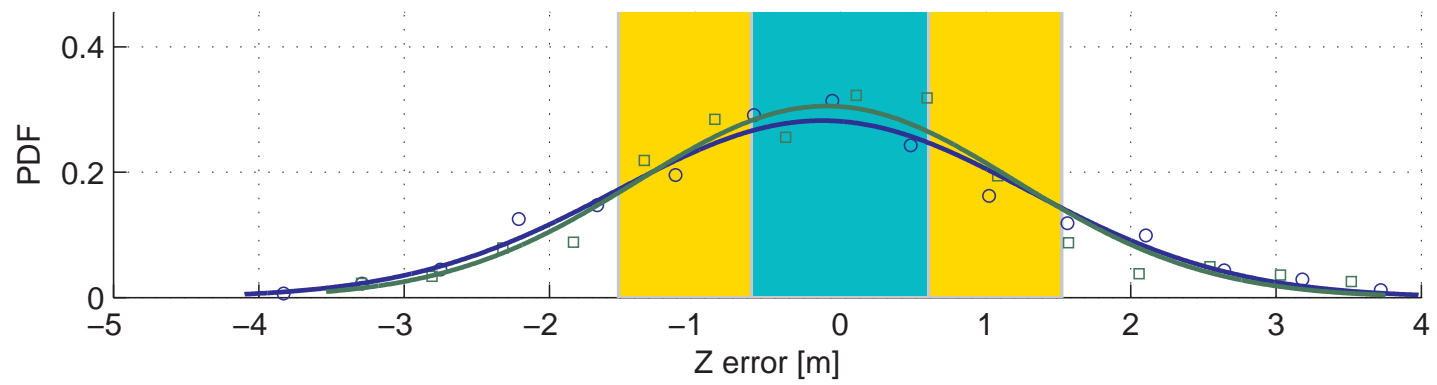

Figure 5.31. Currently Employed Q, R: Gaussian Probability Density Function Controller Comparison for Nonlinear Simulation while Tanker Performs a 15 Degree Right Bank in Non-Steady Atmosphere. 
With a non-steady prevailing wind active, a unidirectional tanker maneuver does not suffice for an exhaustive evaluation of the control laws as, most likely, the response characteristics will be asymmetric as a function of turn direction. Therefore, the previous analysis is repeated for a tanker -15 degree turn to the left. Fig. (5.32) and Fig.(5.33) proves this anticipation in that the baseline controller fails in the tracking task (and fails in receiver flight stabilization) with effector saturation occurring as the tanker performs the left turn (as opposed to a successful tracking operation when the tanker turns right). The Friedland controller on the other hand, presented in Fig. (5.34) and Fig.(5.35), succeeds and manages to maintain lateral deviation within $5.5 \mathrm{~m}$ (18 ft) of the commanded refueling position. Finally, the PDF for the Friedland controller is presented in Fig.(5.36), which is considered to demonstrate robustness in that the system maintains deviation from the refueling command box to within $7.5 \mathrm{~m}(24.6 \mathrm{ft})$ across all axes despite the unaccounted wind disturbances. Again, this response may be further improved by including an approximation for winds in the inception of the control law. (Alternatively, the weighting matrices in the performance index may be refined in an attempt to reduce the sensitivity of the closed loop system to inertial wind and turbulence). 

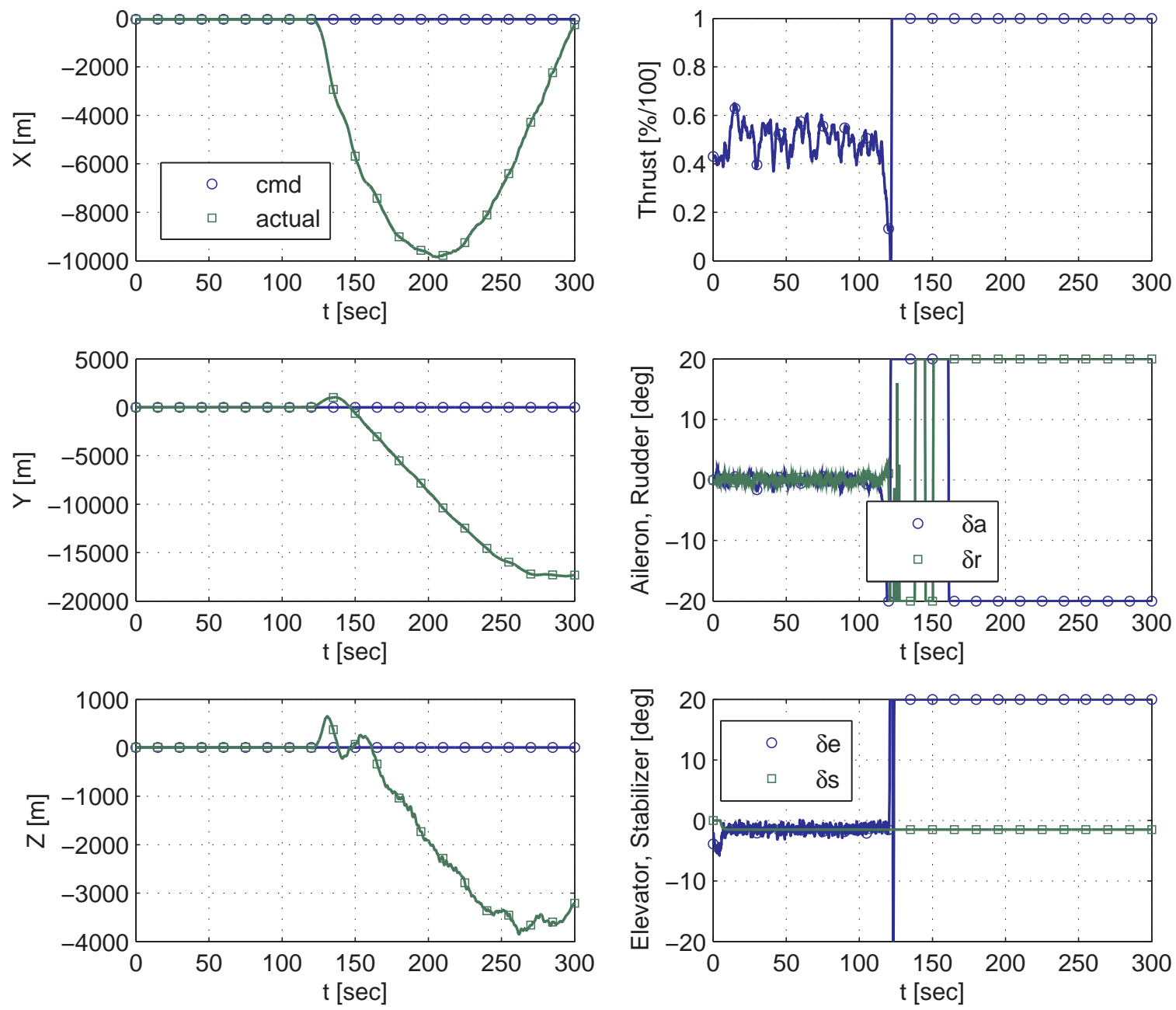

Figure 5.32. Currently Employed Q, R: Station-Keeping and Effectors for Nonlinear Simulation LQR MIMO Control while Tanker Performs a -15 Degree Left Bank in NonSteady Atmosphere. 

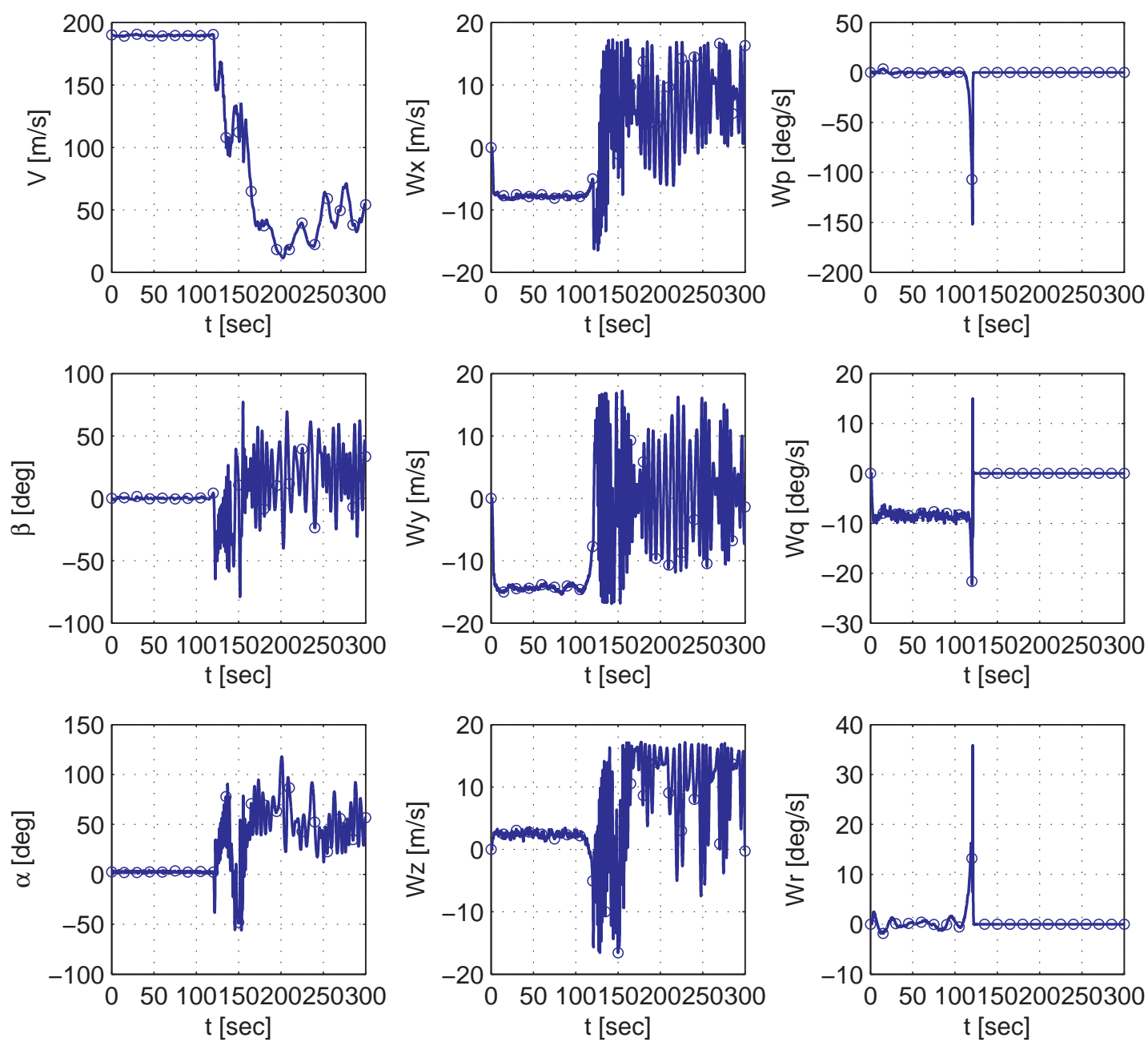

Figure 5.33. Currently Employed Q, R: $V, \beta, \alpha$, and Winds for Nonlinear Simulation LQR MIMO Control while Tanker Performs a -15 Degree Left Bank in Non-Steady Atmosphere. 

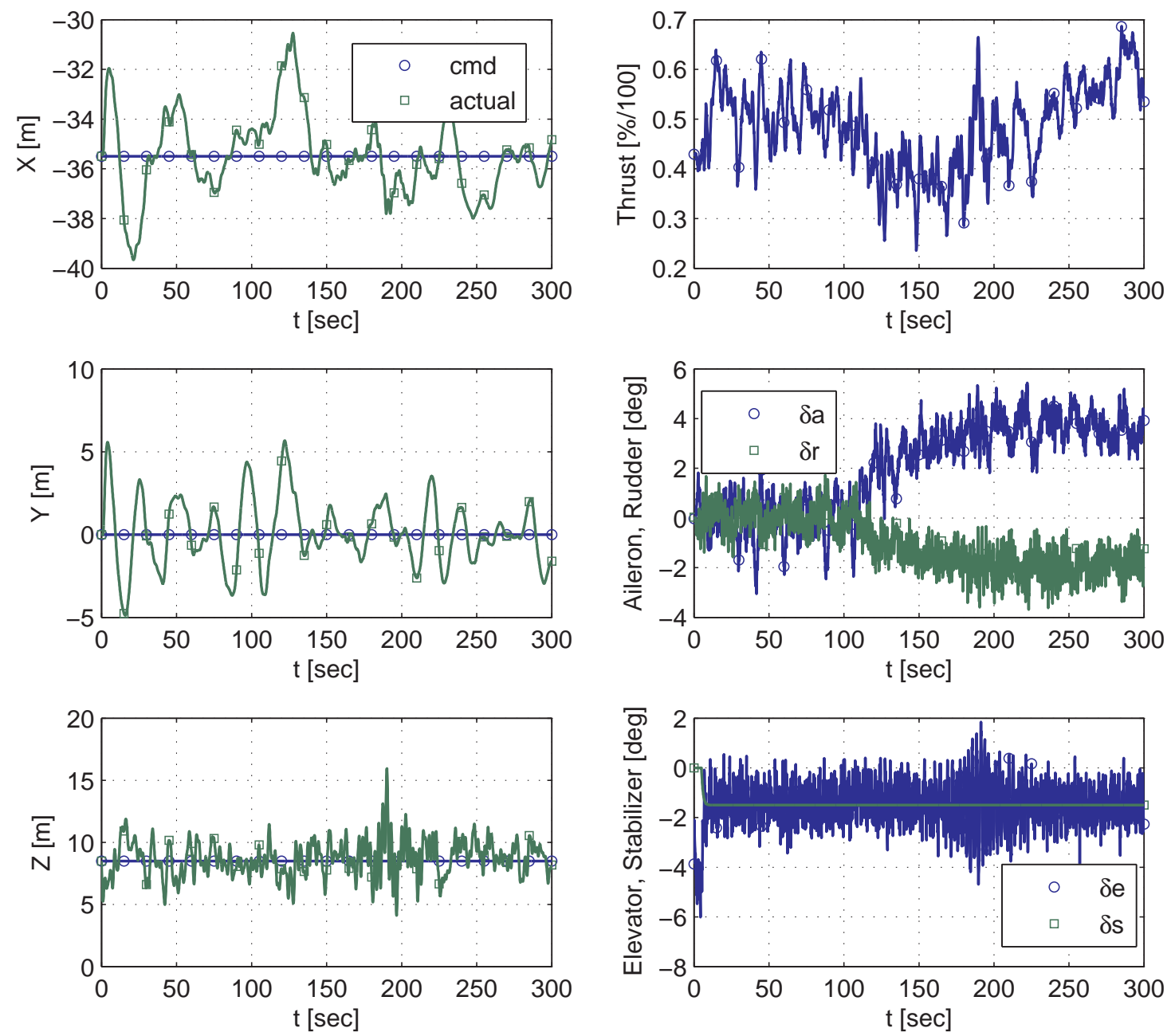

Figure 5.34. Currently Employed Q, R: Station-Keeping and Effectors for Nonlinear Simulation LQR MIMO + Friedland Control while Tanker Performs a -15 Degree Left Bank in Non-Steady Atmosphere. 

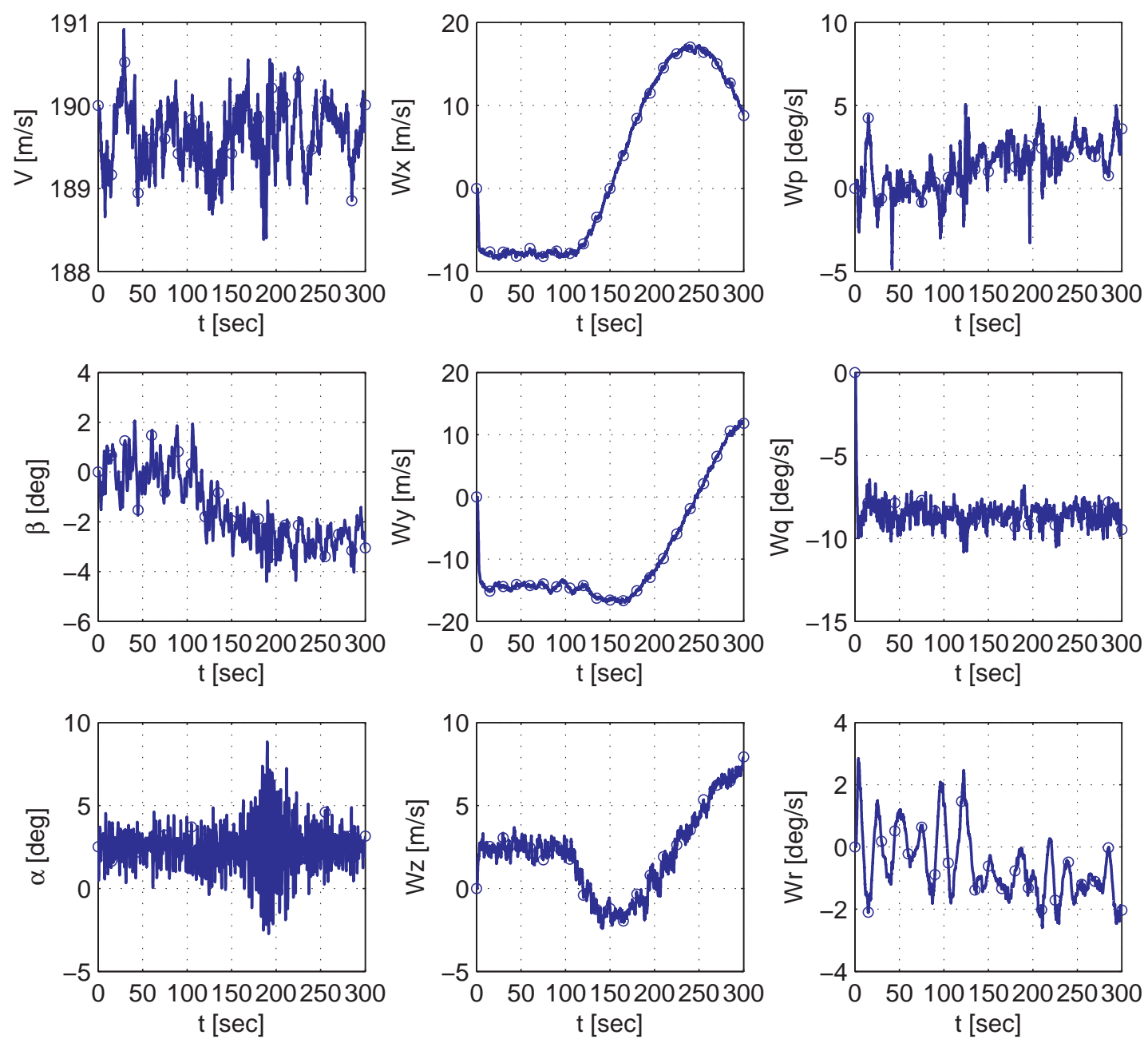

Figure 5.35. Currently Employed Q, R: $V, \beta, \alpha$, and Winds for Nonlinear Simulation LQR MIMO + Friedland Control while Tanker Performs a -15 Degree Left Bank in Non-Steady Atmosphere. 

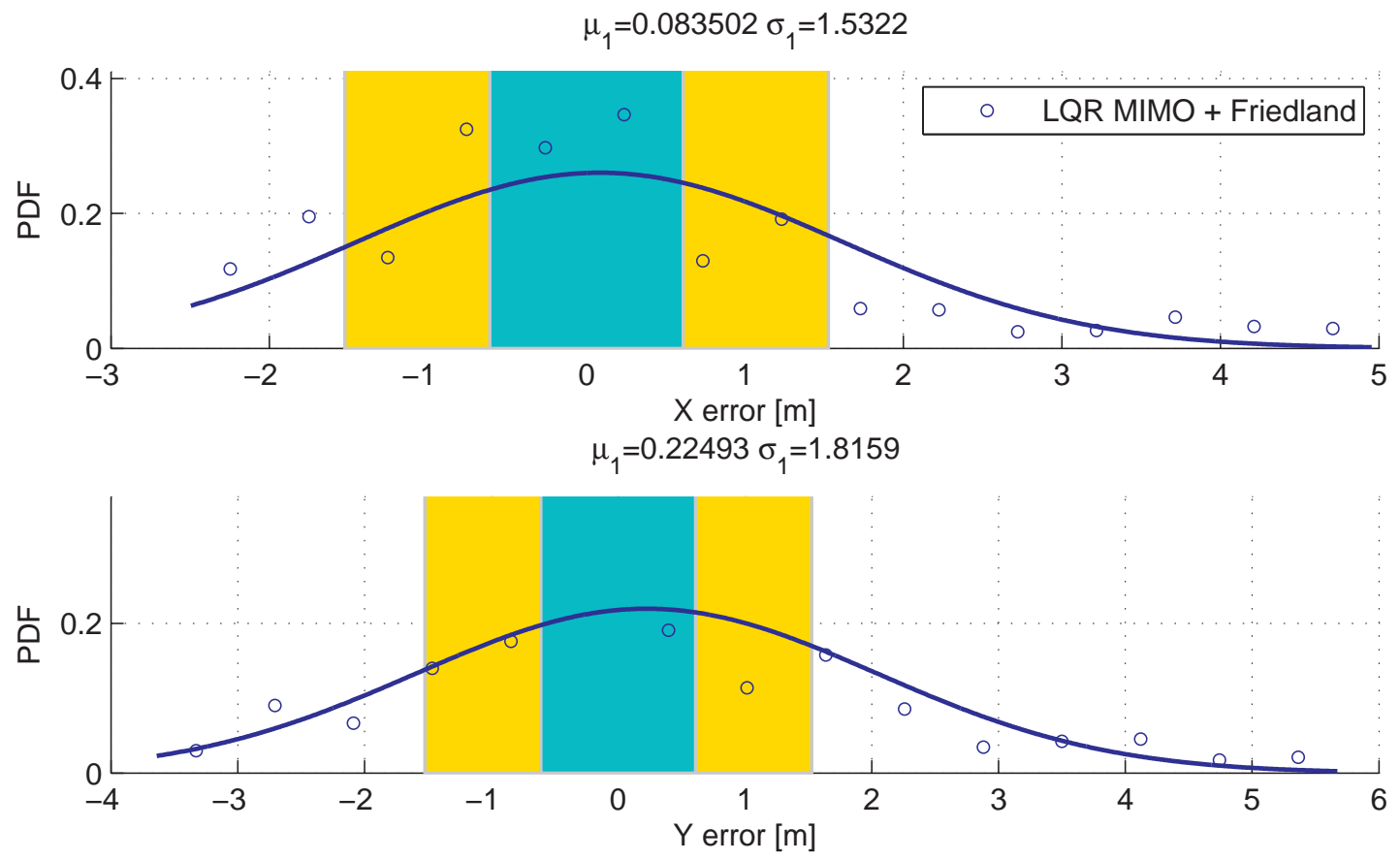

$\mu_{1}=0.29027 \sigma_{1}=1.4346$

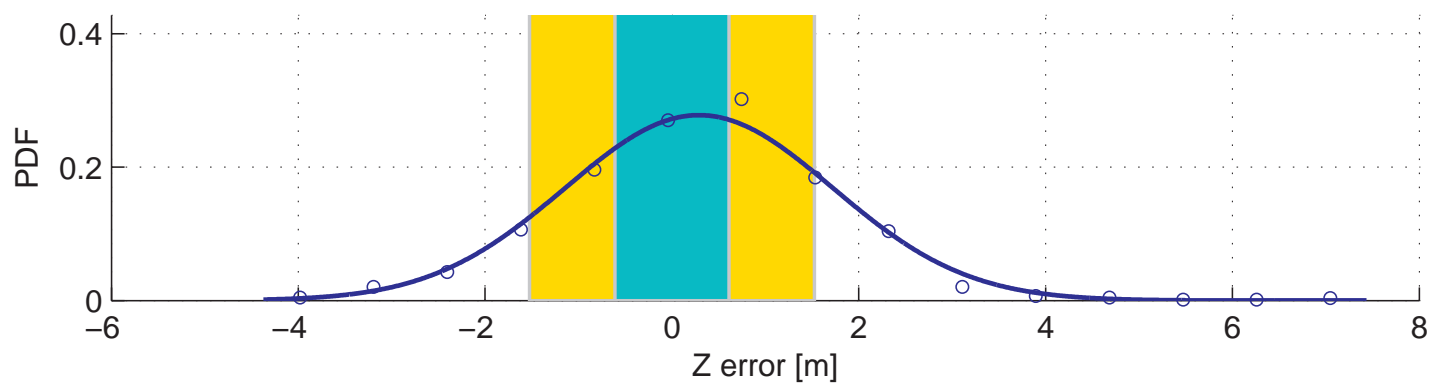

Figure 5.36. Currently Employed Q, R: Gaussian Probability Density Function LQR MIMO + Friedland Control Only for Nonlinear Simulation while Tanker Performs a -15 Degree Left Bank in Non-Steady Atmosphere. 


\section{CHAPTER 6}

\section{SUMMARY, CONCLUSIONS, AND RECOMMENDATIONS FOR FUTURE WORK}

\subsection{Summary}

Table 6.1 presents a summary of the nonlinear simulation results generated in Chapter 5 and allows rapid comparison between both controllers and their ability to maintain contact position in the refueling zone. While in some cases, slight degradation is observed in $x$ and $z$ tracking, the new Friedland disturbance rejection consistently offers significant potential in improving lateral deviation due to an accelerating tanker. Also, in cases where the baseline architecture fails, the new controllers appears to offer an increase in robustness in that stability is preserved. Note these simulation evaluations are repeated for the receiver at the observation relative position in Appendix E.

Table 6.1. Summary of Results for Nonlinear Simulation with Receiver at Contact Position

\begin{tabular}{|c|c|c|c|c|c|c|c|}
\hline Winds & Maneuver & Control & Gain Set & Stable? & Max $\left|e_{x}\right|$ & Max $\left|e_{y}\right|$ & Max $\left|e_{z}\right|$ \\
\hline \hline Vortex Induced & $30 \mathrm{deg}$ & Base & Unity & No & - & - & - \\
\hline Vortex Induced & $30 \mathrm{deg}$ & Friedland & Unity & No & - & - & - \\
\hline Vortex Induced & $30 \mathrm{deg}$ & Base & Base & Yes & $3 \mathrm{~m}$ & $25 \mathrm{~m}$ & $2 \mathrm{~m}$ \\
\hline Vortex Induced & $30 \mathrm{deg}$ & Friedland & Base & Yes & $4.5 \mathrm{~m}$ & $7 \mathrm{~m}$ & $1.5 \mathrm{~m}$ \\
\hline Vortex Induced & $30 \mathrm{deg}$ & Base & Revised & No & - & - & - \\
\hline Vortex Induced & $30 \mathrm{deg}$ & Friedland & Revised & Yes & $0.5 \mathrm{~m}$ & $0.4 \mathrm{~m}$ & $0.5 \mathrm{~m}$ \\
\hline All & $15 \mathrm{deg}$ & Base & Base & Yes & $3.5 \mathrm{~m}$ & $12 \mathrm{~m}$ & $4 \mathrm{~m}$ \\
\hline All & $15 \mathrm{deg}$ & Friedland & Base & Yes & $4 \mathrm{~m}$ & $6 \mathrm{~m}$ & $4 \mathrm{~m}$ \\
\hline All & $-15 \mathrm{deg}$ & Base & Base & No & - & - & - \\
\hline All & $-15 \mathrm{deg}$ & Friedland & Base & Yes & $5 \mathrm{~m}$ & $6 \mathrm{~m}$ & $7 \mathrm{~m}$ \\
\hline
\end{tabular}




\subsection{Conclusions}

Using the equations of motion defined for a receiver vehicle with respect to an accelerating and rotating reference frame (tanker vehicle), developed in prior work [1, $9,10]$, the tanker motion is characterized as disturbance in a two-vehicle metastatespace open loop dynamics time variant system. Utilizing the existing LQR MIMO [1, $9,10]$ infrastructure in place for the receiver, a matrix transformation is developed to map the existing tanker states to receiver disturbance by using the tanker closed loop dynamics equation in conjunction with a rotation matrix (from inertial frame to tanker body frame). The metastate-space open loop system is proved to be time invariant due to the second property of a special orthogonal rotation matrix and allows the formulation of an augmented metastate-space system whose state matrix contains the disturbance quantification aforementioned. The Kalman gain solution of the steady state Algebraic Ricatti Equation is utilized for integration into a suboptimal controller, as proposed by Friedland, and multiple performance indices are analyzed for the loop closure, both linearly and nonlinearly (with aerodynamic coupling between the two bodies and with and without realistic atmospheric conditions). Simulation results exhibit the potential for significant improvement in y-lateral deviation from the refueling box with the Friedland control law enabled. With an optimized performance index to prevent effector saturation of the receiver, both transient and steady state responses are superior with the new feedforward control law, where improved disturbance attenuation on the station-keeping task is clearly visualized in the frequency domain linear analysis.

\subsection{Future Work}

The greatest disturbances on the receiver system, in the context of the stationkeeping aerial refueling task, are (i) accelerations of the target tanker and (ii) the exter- 
nal wind environment in the neighborhood of the receiver spatial position. This research effort has shown that if the item (i) disturbance is modeled linearly for the control law formulation procedure, the Friedland disturbance rejection technique [20] offers significant improvement potential in attenuating the impact of the disturbance on the tracking system. Therefore, a future investigative effort is recommended to expand the Friedland disturbance rejection control for item (ii). As previously aforementioned, this expansion will require approximation of the wind on the receiver state-space system via the (E) disturbance matrix. One possible approach recommended is to estimate the maximum bounds of the wind disturbance and essentially employ a robust control theory by quantifying these approximations. Additionally, the bounded wind estimations will require approximation of the wind rates and state matrix governing the wind dynamics, in order to formulate the metastate-space system with the encapsulated exogenous system.

The procedure for developing the linear transformation matrix from the tanker state vector to receiver disturbance vector can be simplified by revisiting the receiver Equations Of Motion (EOM) for Translational Kinematics (TK). If the tanker TK EOM is substituted directly into the receiver TK EOM, time variance in the rotation matrix, posed by time variation of heading, is canceled by multiplying the transpose of the same rotation matrix. Note this procedure will modify the definition of the receiver disturbance vector, $\underline{w}$, and will modify the tanker translational velocity components to be represented in the tanker wind frame, rather than the inertial frame.

In the linear analysis results, the unity performance index time domain step responses appear to be limited in bandwidth by the feed-forward command dynamics employed in the tanker bank attitude controller. In other words, despite an evenly penalized control action (and without actuator dynamics), the minimum achievable lateral deviation settling time is considered partly tied to the first order lag filters used as command 
conditioning in the tanker bank controller. An alternative feed-forward path in the tanker could be investigated for improving receiver station-keeping transient response.

Nonlinear simulation results were presented with failed station-keeping tasks in which control effectors were recognizably saturated. Future investigation is required to understand the nature of this saturation and whether the saturation causes divergent station-keeping or vice versa (the unstable divergent station-keeping leads to a resultant effector saturation). One, the control action penalty matrix may require refinement in order to lessen the tendency to encounter rate and position limits on the actuators. However, a second consideration to investigate is the validity of the linear model. If the linear model poorly represents the nonlinear model, the controller may fail to stabilize the system. A candidate procedure for validation that should be considered is a time domain comparison to a small perturbation for both systems. In other words, monitor the state and output response of both the linear model and the nonlinear model at an equivalent nominal condition for an equivalent input, usually an impulse of small magnitude. If the nonlinear system remains near the nominal condition sufficiently, good correlation should exist between the time histories if the linear model is a valid approximation. (Note this validity test obviously requires analysis about a stable nominal condition. Otherwise, stimulus by an impulse should drive both system models away from the nominal condition, effectively eliminating the capability to perform a time history comparison).

While the current nonlinear model does not include actuator dynamics, also recommended for potential future work, other items that should be investigated for incorporation into the linear model are winds (as aforementioned, this disturbance may significantly alter the nominal conditions and should also be considered in the trim algorithm or computation of the nominal condition); the effects of a non-zero stabilizer (this control surface position should be proved negligible if not incorporated into the 
control formulation); and the dynamics of the engine (clearly the controller relies on an accurate depiction of the thrust magnitude and frequency response in optimizing the linear quadratic regulator solution. Note the nonlinear simulation uses a first order engine dynamics model and is not captured in the linear model). Additionally, the current gain scheduling for the controllers in the nonlinear simulation includes only six nominal conditions. Considering additional nominal conditions and independent variables may offer an increase to the envelope of operation for the controllers or an increase in robustness to the presence of model uncertainties.

Nonlinear simulation results were presented with the receiver angle of sideslip noticeably increasing throughout the turning maneuver. As previously mentioned, significant values of sideslip angle in banked flight may be considered as unfavorable for coordinated turning. This response is recommended as a potential tuning effort to be conducted in future performance indices. Furthermore, the oscillations of angle of attack, as well as questionable bifurcations in the responses of control surfaces observed in the time histories, should also be understood and investigated as potential stability issues.

Regarding stability, a number of classical Single Input Single Output (SISO) linear analysis results were presented. In a conventional study, future work could be to extract stability margins per effector for each control law and nominal condition. However, modern optimal control demands the investigation of Multiple Input Multiple Output (MIMO) stability. Future work is recommended to investigate quantifying stability margins for a MIMO system, with singular value theory and multi-variable frequency domain analysis.

Multiple performance indices were presented and used to define the cost function in the linear quadratic regulator minimization problem. The unity performance index aside, other weight factors were established after innumerable trial simulation executions, where the process involved time history analysis followed by a reiteration to the performance 
index. Obviously, this process can be burdensome for establishing an optimal gain set. Future work is recommended to investigate methods of automation for refinement of the performance index. Consideration should include an attempt to grasp the effective impact on the controller with a weight modification. In other words, future work may identify and correlate classical design techniques to the performance index refinement. For example, if one increases the weight on the receiver state deviation, $y$, without modifying the weight factor for error state deviation of the same variable, $e_{y}$, ascertain if this performance index refinement essentially modifies the proportional to integral ratio in the controller architecture (by determining if a similar response is achieved by utilizing the classical technique of modifying this ratio in a $P+I$ control law). Finally, regarding control law architecture, other methodologies should be investigated in future work (feedback linearization, sliding mode control, adaptive control, robust control, etc.) as well as reconsideration of the assumption that all states are available for feedback in the current controller (i.e. incorporation of observers with linear-quadratic Gaussian control, etc.). Furthermore, investigations could also be extended to nonlinear theory in order to determine whether Jacobian linearization is a sufficient tool for analyzing the stability of the aerial refueling problem. Alternatives may include examination of potential Lyapunov function candidates and their application to guaranteed stability theory. 
APPENDIX A

EXPANDED SCALAR EQUATIONS OF MOTION FOR RECEIVER AIRCRAFT IN THE ABSENCE OF WINDS 
This appendix lists the receiver expanded scalar equations of motion presented in matrix-form in Chapter 2 without the wind terms. The wind terms are excluded in order to provide a reference to the form of nonlinear equations utilized in the linearization procedure for the receiver control law formulation.

\section{A.1 Translational Kinematics}

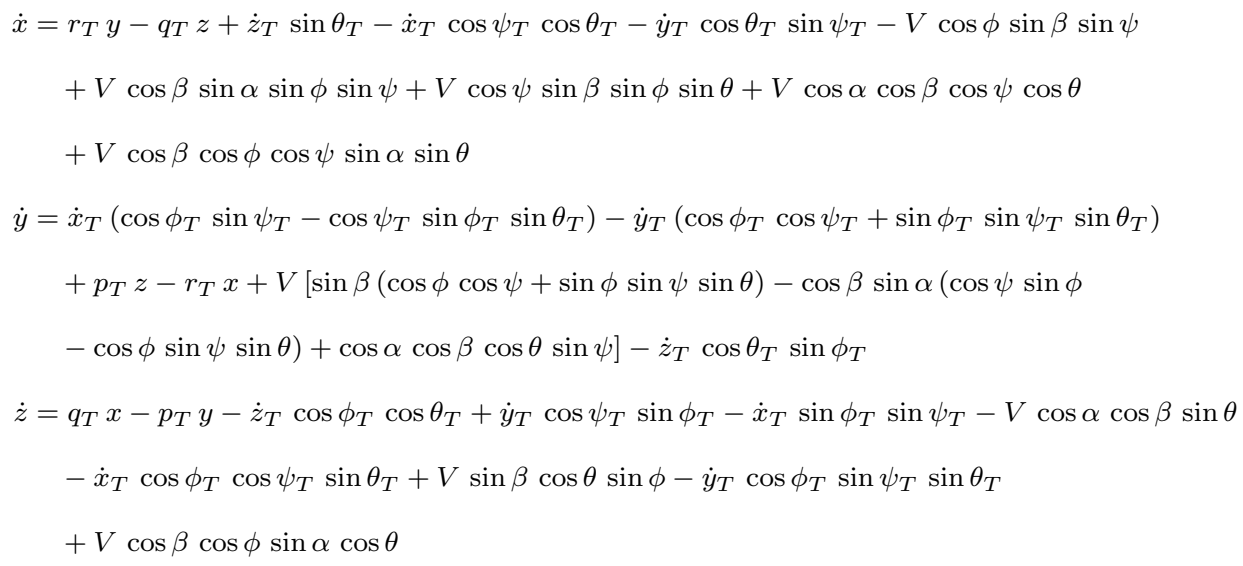

\section{A.2 Translational Dynamics}

$$
\begin{aligned}
\dot{V}= & \left(T_{y} \sin \beta-D+T_{x} \cos \alpha \cos \beta+T_{z} \cos \beta \sin \alpha\right) / m_{R}+g \cos \phi \sin \beta \sin \psi \sin \theta_{T} \\
& +g \cos \phi_{T} \sin \beta \cos \theta_{T} \cos \theta \sin \phi-g \cos \beta \sin \alpha \sin \phi \sin \psi \sin \theta_{T} \\
& -g \cos \psi \sin \beta \sin \phi \sin \theta_{T} \sin \theta-g \cos \alpha \cos \beta \cos \phi_{T} \cos \theta_{T} \sin \theta \\
& -g \cos \alpha \cos \beta \cos \psi \cos \theta \sin \theta_{T}+g \cos \phi \cos \psi \sin \beta \cos \theta_{T} \sin \phi_{T} \\
& +g \sin \beta \cos \theta_{T} \sin \phi \sin \phi_{T} \sin \psi \sin \theta+g \cos \beta \cos \phi \cos \phi_{T} \sin \alpha \cos \theta_{T} \cos \theta \\
& +g \cos \alpha \cos \beta \cos \theta_{T} \cos \theta \sin \phi_{T} \sin \psi-g \cos \beta \cos \psi \sin \alpha \cos \theta_{T} \sin \phi \sin \phi_{T} \\
& -g \cos \beta \cos \phi \cos \psi \sin \alpha \sin \theta_{T} \sin \theta \\
& +g \cos \beta \cos \phi \sin \alpha \cos \theta_{T} \sin \phi_{T} \sin \psi \sin \theta \\
\dot{\beta}= & p \sin \alpha-r \cos \alpha-S /\left(V m_{R}\right)-r_{T} \sin \alpha \sin \theta-r_{T} \cos \alpha \cos \phi \cos \theta+q_{T} \cos \alpha \cos \psi \sin \phi \\
& +p_{T} \cos \psi \sin \alpha \cos \theta-p_{T} \cos \alpha \sin \phi \sin \psi+q_{T} \sin \alpha \cos \theta \sin \psi+\left(T_{y} \cos \beta\right) /\left(V m_{R}\right) \\
& -p_{T} \cos \alpha \cos \phi \cos \psi \sin \theta-q_{T} \cos \alpha \cos \phi \sin \psi \sin \theta-\left(T_{x} \cos \alpha \sin \beta\right) /\left(V m_{R}\right) \\
& -\left(T_{z} \sin \alpha \sin \beta\right) /\left(V m_{R}\right)+\left(g \cos \beta \cos \phi \sin \psi \sin \theta_{T}\right) / V
\end{aligned}
$$


$+\left(g \cos \beta \cos \phi \cos \psi \cos \theta_{T} \sin \phi_{T}\right) / V+\left(g \cos \beta \cos \phi_{T} \cos \theta_{T} \cos \theta \sin \phi\right) / V$

$+\left(g \cos \alpha \cos \phi_{T} \sin \beta \cos \theta_{T} \sin \theta\right) / V+\left(g \cos \alpha \cos \psi \sin \beta \cos \theta \sin \theta_{T}\right) / V$

$-\left(g \cos \beta \cos \psi \sin \phi \sin \theta_{T} \sin \theta\right) / V+\left(g \sin \alpha \sin \beta \sin \phi \sin \psi \sin \theta_{T}\right) / V$

$-\left(g \cos \phi \cos \phi_{T} \sin \alpha \sin \beta \cos \theta_{T} \cos \theta\right) / V-\left(g \cos \alpha \sin \beta \cos \theta_{T} \cos \theta \sin \phi_{T} \sin \psi\right) / V$

$+\left(g \cos \psi \sin \alpha \sin \beta \cos \theta_{T} \sin \phi \sin \phi_{T}\right) / V+\left(g \cos \phi \cos \psi \sin \alpha \sin \beta \sin \theta_{T} \sin \theta\right) / V$

$+\left(g \cos \beta \cos \theta_{T} \sin \phi \sin \phi_{T} \sin \psi \sin \theta\right) / V$

$-\left(g \cos \phi \sin \alpha \sin \beta \cos \theta_{T} \sin \phi_{T} \sin \psi \sin \theta\right) / V$

$\dot{\alpha}=q-p \cos \alpha \tan \beta+q_{T} \cos \phi \cos \psi-r \sin \alpha \tan \beta-p_{T} \cos \phi \sin \psi+r_{T} \cos \theta \sin \phi$

$-L /\left(V m_{R} \cos \beta\right)+r_{T} \cos \alpha \tan \beta \sin \theta+p_{T} \cos \psi \sin \phi \sin \theta+q_{T} \sin \phi \sin \psi \sin \theta$

$-p_{T} \cos \alpha \cos \psi \tan \beta \cos \theta+\left(T_{z} \cos \alpha\right) /\left(V m_{R} \cos \beta\right)-q_{T} \cos \alpha \tan \beta \cos \theta \sin \psi$

$-r_{T} \cos \phi \sin \alpha \tan \beta \cos \theta-\left(T_{x} \sin \alpha\right) /\left(V m_{R} \cos \beta\right)+q_{T} \cos \psi \sin \alpha \tan \beta \sin \phi$

$-p_{T} \sin \alpha \tan \beta \sin \phi \sin \psi-p_{T} \cos \phi \cos \psi \sin \alpha \tan \beta \sin \theta$

$-q_{T} \cos \phi \sin \alpha \tan \beta \sin \psi \sin \theta+\left(g \cos \phi_{T} \sin \alpha \cos \theta_{T} \sin \theta\right) /(V \cos \beta)$

$+\left(g \cos \psi \sin \alpha \cos \theta \sin \theta_{T}\right) /(V \cos \beta)-\left(g \cos \alpha \sin \phi \sin \psi \sin \theta_{T}\right) /(V \cos \beta)$

$+\left(g \cos \alpha \cos \phi \cos \phi_{T} \cos \theta_{T} \cos \theta\right) /(V \cos \beta)-\left(g \cos \alpha \cos \psi \cos \theta_{T} \sin \phi \sin \phi_{T}\right) /(V \cos \beta)$

$-\left(g \cos \alpha \cos \phi \cos \psi \sin \theta_{T} \sin \theta\right) /(V \cos \beta)-\left(g \sin \alpha \cos \theta_{T} \cos \theta \sin \phi_{T} \sin \psi\right) /(V \cos \beta)$

$+\left(g \cos \alpha \cos \phi \cos \theta_{T} \sin \phi_{T} \sin \psi \sin \theta\right) /(V \cos \beta)$

\section{A.3 Rotational Kinematics}

$$
\begin{aligned}
& \dot{\phi}=p+q \sin \phi \tan \theta+r \cos \phi \tan \theta \\
& \dot{\theta}=q \cos \phi-r \sin \phi \\
& \dot{\psi}=(q \sin \phi+r \cos \phi) \sec \theta
\end{aligned}
$$

\section{A.4 Rotational Dynamics}

$$
\begin{aligned}
\dot{p}= & \left(\left\{I _ { x z } ^ { 2 } \left[q-p_{T}(\cos \phi \sin \psi-\cos \psi \sin \phi \sin \theta)\right.\right.\right. \\
& \left.\left.+q_{T}(\cos \phi \cos \psi+\sin \phi \sin \psi \sin \theta)+r_{T} \cos \theta \sin \phi\right]\right\} /\left(I_{x z}^{2}-I_{x x} I_{z z}\right) \\
& +\left\{I _ { z z } ^ { 2 } \left[q-p_{T}(\cos \phi \sin \psi-\cos \psi \sin \phi \sin \theta)+q_{T}(\cos \phi \cos \psi\right.\right. \\
& \left.\left.\left.+\sin \phi \sin \psi \sin \theta)+r_{T} \cos \theta \sin \phi\right]\right\} /\left(I_{x z}^{2}-I_{x x} I_{z z}\right)\right)\left[r+p_{T}(\sin \phi \sin \psi+\cos \phi \cos \psi \sin \theta)\right. \\
& \left.-q_{T}(\cos \psi \sin \phi-\cos \phi \sin \psi \sin \theta)+r_{T} \cos \phi \cos \theta\right] \\
& +r_{T}(q \cos \phi \cos \theta-r \cos \theta \sin \phi)+\dot{r}_{T} \sin \theta
\end{aligned}
$$


$-\left(\left\{I_{x x} I_{x z}\left[q-p_{T}(\cos \phi \sin \psi-\cos \psi \sin \phi \sin \theta)\right.\right.\right.$

$\left.\left.+q_{T}(\cos \phi \cos \psi+\sin \phi \sin \psi \sin \theta)+r_{T} \cos \theta \sin \phi\right]\right\} /\left(I_{x z}^{2}-I_{x x} I_{z z}\right)$

$+\left\{I_{x z} I_{z z}\left[q-p_{T}(\cos \phi \sin \psi-\cos \psi \sin \phi \sin \theta)+q_{T}(\cos \phi \cos \psi\right.\right.$

$\left.\left.\left.+\sin \phi \sin \psi \sin \theta)+r_{T} \cos \theta \sin \phi\right]\right\} /\left(I_{x z}^{2}-I_{x x} I_{z z}\right)\right)\left(p-r_{T} \sin \theta\right.$

$\left.+p_{T} \cos \psi \cos \theta+q_{T} \cos \theta \sin \psi\right)+p_{T}[q(\sin \phi \sin \psi$

$+\cos \phi \cos \psi \sin \theta)+r(\cos \phi \sin \psi-\cos \psi \sin \phi \sin \theta)]$

$-q_{T}[q(\cos \psi \sin \phi-\cos \phi \sin \psi \sin \theta)+r(\cos \phi \cos \psi$

$+\sin \phi \sin \psi \sin \theta)]-\left(I_{z z} \mathcal{L}\right) /\left(I_{x z}^{2}-I_{x x} I_{z z}\right)-\left(I_{x z} \mathcal{N}\right) /\left(I_{x z}^{2}-I_{x x} I_{z z}\right)$

$+I_{y y}\left(\left[I_{x z}\left(p-r_{T} \sin \theta+p_{T} \cos \psi \cos \theta+q_{T} \cos \theta \sin \psi\right)\right] /\left(I_{x z}^{2}-I_{x x} I_{z z}\right)\right.$

$-\left\{I_{z z}\left[r+p_{T}(\sin \phi \sin \psi+\cos \phi \cos \psi \sin \theta)-q_{T}(\cos \psi \sin \phi\right.\right.$

$\left.\left.\left.-\cos \phi \sin \psi \sin \theta)+r_{T} \cos \phi \cos \theta\right]\right\} /\left(I_{x z}^{2}-I_{x x} I_{z z}\right)\right)\left[q-p_{T}(\cos \phi \sin \psi\right.$

$-\cos \psi \sin \phi \sin \theta)+q_{T}(\cos \phi \cos \psi+\sin \phi \sin \psi \sin \theta)$

$\left.+r_{T} \cos \theta \sin \phi\right]-\dot{p}_{T} \cos \psi \cos \theta$

$-\dot{q}_{T} \cos \theta \sin \psi$

$\dot{q}=q_{T}[p(\cos \psi \sin \phi-\cos \phi \sin \psi \sin \theta)+r \cos \theta \sin \psi]+\dot{p}_{T}(\cos \phi \sin \psi-\cos \psi \sin \phi \sin \theta)$

$-\dot{q}_{T}(\cos \phi \cos \psi+\sin \phi \sin \psi \sin \theta)+\mathcal{M} / I_{y y}$

$-\left(\left\{I_{x x}\left[r+p_{T}(\sin \phi \sin \psi+\cos \phi \cos \psi \sin \theta)-q_{T}(\cos \psi \sin \phi\right.\right.\right.$

$\left.\left.-\cos \phi \sin \psi \sin \theta)+r_{T} \cos \phi \cos \theta\right]\right\} / I_{y y}+\left[I_{x z}\left(p-r_{T} \sin \theta\right.\right.$

$\left.\left.\left.+p_{T} \cos \psi \cos \theta+q_{T} \cos \theta \sin \psi\right)\right] / I_{y y}\right)\left(p-r_{T} \sin \theta+p_{T} \cos \psi \cos \theta\right.$

$\left.+q_{T} \cos \theta \sin \psi\right)-r_{T}(r \sin \theta+p \cos \phi \cos \theta)$

$+\left(\left\{I_{x z}\left[r+p_{T}(\sin \phi \sin \psi+\cos \phi \cos \psi \sin \theta)-q_{T}(\cos \psi \sin \phi\right.\right.\right.$

$\left.\left.-\cos \phi \sin \psi \sin \theta)+r_{T} \cos \phi \cos \theta\right]\right\} / I_{y y}+\left[I_{z z}\left(p-r_{T} \sin \theta\right.\right.$

$\left.\left.\left.+p_{T} \cos \psi \cos \theta+q_{T} \cos \theta \sin \psi\right)\right] / I_{y y}\right)\left[r+p_{T}(\sin \phi \sin \psi\right.$

$+\cos \phi \cos \psi \sin \theta)-q_{T}(\cos \psi \sin \phi-\cos \phi \sin \psi \sin \theta)$

$\left.+r_{T} \cos \phi \cos \theta\right]-p_{T}[p(\sin \phi \sin \psi+\cos \phi \cos \psi \sin \theta)$

$-r \cos \psi \cos \theta]-\dot{r}_{T} \cos \theta \sin \phi$

$\dot{r}=q_{T}[p(\cos \phi \cos \psi+\sin \phi \sin \psi \sin \theta)-q \cos \theta \sin \psi]+\left(\left\{I_{x x} I_{x z}\left[q-p_{T}(\cos \phi \sin \psi-\cos \psi \sin \phi \sin \theta)\right.\right.\right.$

$\left.\left.+q_{T}(\cos \phi \cos \psi+\sin \phi \sin \psi \sin \theta)+r_{T} \cos \theta \sin \phi\right]\right\} /\left(I_{x z}^{2}-I_{x x} I_{z z}\right)$

$+\left\{I_{x z} I_{z z}\left[q-p_{T}(\cos \phi \sin \psi-\cos \psi \sin \phi \sin \theta)+q_{T}(\cos \phi \cos \psi\right.\right.$

$\left.\left.\left.+\sin \phi \sin \psi \sin \theta)+r_{T} \cos \theta \sin \phi\right]\right\} /\left(I_{x z}^{2}-I_{x x} I_{z z}\right)\right)\left[r+p_{T}(\sin \phi \sin \psi\right.$

$+\cos \phi \cos \psi \sin \theta)-q_{T}(\cos \psi \sin \phi-\cos \phi \sin \psi \sin \theta)$

$\left.+r_{T} \cos \phi \cos \theta\right]-\dot{p}_{T}(\sin \phi \sin \psi+\cos \phi \cos \psi \sin \theta)$

$+\dot{q}_{T}(\cos \psi \sin \phi-\cos \phi \sin \psi \sin \theta)+r_{T}(q \sin \theta+p \cos \theta \sin \phi)$ 
$-\left(\left\{I_{x x}^{2}\left[q-p_{T}(\cos \phi \sin \psi-\cos \psi \sin \phi \sin \theta)+q_{T}(\cos \phi \cos \psi\right.\right.\right.$

$\left.\left.+\sin \phi \sin \psi \sin \theta)+r_{T} \cos \theta \sin \phi\right]\right\} /\left(I_{x z}^{2}-I_{x x} I_{z z}\right)+\left\{I_{x z}^{2}\left[q-p_{T}(\cos \phi \sin \psi\right.\right.$

$-\cos \psi \sin \phi \sin \theta)+q_{T}(\cos \phi \cos \psi+\sin \phi \sin \psi \sin \theta)$

$\left.\left.\left.+r_{T} \cos \theta \sin \phi\right]\right\} /\left(I_{x z}^{2}-I_{x x} I_{z z}\right)\right)\left(p-r_{T} \sin \theta+p_{T} \cos \psi \cos \theta+q_{T} \cos \theta \sin \psi\right)$

$-p_{T}[p(\cos \phi \sin \psi-\cos \psi \sin \phi \sin \theta)+q \cos \psi \cos \theta]$

$-\left(I_{x z} \mathcal{L}\right) /\left(I_{x z}^{2}-I_{x x} I_{z z}\right)-\left(I_{x x} \mathcal{N}\right) /\left(I_{x z}^{2}-I_{x x} I_{z z}\right)+I_{y y}\left(\left[I_{x x}\left(p-r_{T} \sin \theta\right.\right.\right.$

$\left.\left.+p_{T} \cos \psi \cos \theta+q_{T} \cos \theta \sin \psi\right)\right] /\left(I_{x z}^{2}-I_{x x} I_{z z}\right)-\left\{I_{x z}\left[r+p_{T}(\sin \phi \sin \psi\right.\right.$

$+\cos \phi \cos \psi \sin \theta)-q_{T}(\cos \psi \sin \phi-\cos \phi \sin \psi \sin \theta)$

$\left.\left.\left.+r_{T} \cos \phi \cos \theta\right]\right\} /\left(I_{x z}^{2}-I_{x x} I_{z z}\right)\right)\left[q-p_{T}(\cos \phi \sin \psi-\cos \psi \sin \phi \sin \theta)\right.$

$\left.+q_{T}(\cos \phi \cos \psi+\sin \phi \sin \psi \sin \theta)+r_{T} \cos \theta \sin \phi\right]$

$-\dot{r}_{T} \cos \phi \cos \theta$ 
APPENDIX B

EXPANDED SCALAR EQUATIONS OF MOTION FOR TANKER AIRCRAFT IN THE ABSENCE OF WINDS 
This appendix lists the tanker expanded scalar equations of motion presented in matrix-form in Chapter 2 without the wind terms. Again, as presented in Appendix A for the receiver, the wind terms are excluded in order to provide a reference to the form of nonlinear equations utilized in the linearization procedure for the tanker control law formulation.

\section{B.1 Translational Kinematics}

$$
\begin{aligned}
\dot{x}_{T}= & V_{T} \cos \beta_{T} \sin \alpha_{T}\left(\sin \phi_{T} \sin \psi_{T}+\cos \phi_{T} \cos \psi_{T} \sin \theta_{T}\right)-V_{T} \sin \beta_{T}\left(\cos \phi_{T} \sin \psi_{T}\right. \\
& \left.-\cos \psi_{T} \sin \phi_{T} \sin \theta_{T}\right)+V_{T} \cos \alpha_{T} \cos \beta_{T} \cos \psi_{T} \cos \theta_{T} \\
\dot{y}_{T}= & V_{T} \sin \beta_{T}\left(\cos \phi_{T} \cos \psi_{T}+\sin \phi_{T} \sin \psi_{T} \sin \theta_{T}\right)-V_{T} \cos \beta_{T} \sin \alpha_{T}\left(\cos \psi_{T} \sin \phi_{T}\right. \\
& \left.-\cos \phi_{T} \sin \psi_{T} \sin \theta_{T}\right)+V_{T} \cos \alpha_{T} \cos \beta_{T} \cos \theta_{T} \sin \psi_{T} \\
\dot{z}_{T}= & V_{T} \sin \beta_{T} \cos \theta_{T} \sin \phi_{T}-V_{T} \cos \alpha_{T} \cos \beta_{T} \sin \theta_{T}+V_{T} \cos \beta_{T} \cos \phi_{T} \sin \alpha_{T} \cos \theta_{T}
\end{aligned}
$$

\section{B.2 Translational Dynamics}

$$
\begin{aligned}
\dot{V}_{T}= & g \sin \beta_{T} \cos \theta_{T} \sin \phi_{T}-g \cos \alpha_{T} \cos \beta_{T} \sin \theta_{T}-\left(D_{T}-T_{T} \cos \alpha_{T} \cos \beta_{T} \cos \delta_{T}\right. \\
& \left.+T_{T} \cos \beta_{T} \sin \alpha_{T} \sin \delta_{T}\right) / m_{T}+g \cos \beta_{T} \cos \phi_{T} \sin \alpha_{T} \cos \theta_{T} \\
\dot{\beta}_{T}= & -\left(S_{T}+T_{T} \cos \alpha_{T} \cos \delta_{T} \sin \beta_{T}-T_{T} \sin \alpha_{T} \sin \beta_{T} \sin \delta_{T}+V_{T} m_{T} r_{T} \cos \alpha_{T}\right. \\
& -V_{T} m_{T} p_{T} \sin \alpha_{T}-g m_{T} \cos \beta_{T} \cos \theta_{T} \sin \phi_{T}-g m_{T} \cos \alpha_{T} \sin \beta_{T} \sin \theta_{T} \\
& \left.+g m_{T} \cos \phi_{T} \sin \alpha_{T} \sin \beta_{T} \cos \theta_{T}\right) /\left(V_{T} m_{T}\right) \\
\dot{\alpha}_{T}= & -\left(L_{T}+T_{T} \cos \alpha_{T} \sin \delta_{T}+T_{T} \cos \delta_{T} \sin \alpha_{T}-V_{T} m_{T} q_{T} \cos \beta_{T}-g m_{T} \sin \alpha_{T} \sin \theta_{T}\right. \\
& -g m_{T} \cos \alpha_{T} \cos \phi_{T} \cos \theta_{T}+V_{T} m_{T} r_{T} \cos \beta_{T} \sin \alpha_{T} \tan \beta_{T} \\
& \left.+V_{T} m_{T} p_{T} \cos \alpha_{T} \cos \beta_{T} \tan \beta_{T}\right) /\left(V_{T} m_{T} \cos \beta_{T}\right)
\end{aligned}
$$

\section{B.3 Rotational Kinematics}

$$
\begin{aligned}
& \dot{\phi}_{T}=p_{T}+q_{T} \sin \phi_{T} \tan \theta_{T}+r_{T} \cos \phi_{T} \tan \theta_{T} \\
& \dot{\theta}_{T}=q_{T} \cos \phi_{T}-r_{T} \sin \phi_{T} \\
& \dot{\psi}_{T}=\left(q_{T} \sin \phi_{T}+r_{T} \cos \phi_{T}\right) \sec \theta_{T}
\end{aligned}
$$


B.4 Rotational Dynamics

$$
\begin{aligned}
\dot{p}_{T}= & -\left(I_{z z_{T}} \mathcal{L}_{T}+I_{x z_{T}} \mathcal{N}_{T}-I_{x z_{T}}^{2} q_{T} r_{T}-I_{z z_{T}}^{2} q_{T} r_{T}+I_{x x_{T}} I_{x z_{T}} p_{T} q_{T}-I_{x z_{T}} I_{y y_{T}} p_{T} q_{T}\right. \\
& \left.+I_{x z_{T}} I_{z z_{T}} p_{T} q_{T}+I_{y y_{T}} I_{z z_{T}} q_{T} r_{T}\right) /\left(I_{x z_{T}}^{2}-I_{x x_{T}} I_{z z_{T}}\right) \\
\dot{q}_{T}= & \left(\mathcal{M}_{T}-I_{x z_{T}} p_{T}^{2}+I_{x z_{T}} r_{T}^{2}-I_{x x_{T}} p_{T} r_{T}+I_{z z_{T}} p_{T} r_{T}\right) / I_{y y_{T}} \\
\dot{r}_{T}= & -\left(I_{x z_{T}} \mathcal{L}_{T}+I_{x x_{T}} \mathcal{N}_{T}+I_{x x_{T}}^{2} p_{T} q_{T}+I_{x z_{T}}^{2} p_{T} q_{T}-I_{x x_{T}} I_{y y_{T}} p_{T} q_{T}-I_{x x_{T}} I_{x z_{T}} q_{T} r_{T}\right. \\
& \left.+I_{x z_{T}} I_{y y_{T}} q_{T} r_{T}-I_{x z_{T}} I_{z z_{T}} q_{T} r_{T}\right) /\left(I_{x z_{T}}^{2}-I_{x x_{T}} I_{z z_{T}}\right)
\end{aligned}
$$




\section{APPENDIX C}

CONTINUOUS TIME LINEAR QUADRATIC REGULATOR SUBOPTIMAL SOLUTION 
This appendix solves the Continuous Time Linear Quadratic Regulator (CT LQR), with the Friedland disturbance rejection method, by employing the procedure described in detail by Lewis and Syrmos [20] [27]. The choice of presenting the CT LQR complements the simulation in this work which executes as a continuous time Simulink ${ }^{\circledR}$ model with a fixed step of $\frac{1}{20}$ seconds using the ode3 Bogacki-Shampine solver algorithm.

Recalling the linear system presented in Chapter 2 in Eq.(2.16),

$$
\underline{\dot{x}}_{o}=\mathbf{A}_{\mathbf{o}} \underline{x}_{o}+\mathbf{B}_{\mathbf{o}} \underline{u}
$$

with state matrix $\mathbf{A}_{\mathbf{o}} \in \Re^{28 x 28}$, state vector $\underline{x}_{o} \in \Re^{28 x 1}$, control input matrix $\mathbf{B}_{\mathbf{o}} \in \Re^{28 x 6}$, and control vector $\underline{u} \in \Re^{6 x 1}$, a general performance index or cost, $J$, to be minimized is given as

$$
J=\phi\left(\underline{x}_{o}(T), T\right)+\int_{t_{0}}^{T} L\left(\underline{x}_{o}, \underline{u}, \tau\right) d \tau
$$

where the function, $\phi$, is defined as a quadratic function of values for both the final state vector, $\underline{x}_{o}(T)$, and Ricatti kernel matrix, $\hat{\mathbf{M}}$.

$$
\phi\left(\underline{x}_{o}(T), T\right)=\frac{1}{2} \underline{x}_{o}^{T}(T) \hat{\mathbf{M}}(T) \underline{x}_{o}(T)
$$

The Lagrangian function $L$ within the finite integral component of the performance index in Eq. (C.2) shapes the desired transient response of the closed loop system and is defined as

$$
L\left(\underline{x}_{o}, \underline{u}, \tau\right)=\frac{1}{2}\left[\underline{x}_{o}^{T}(\tau) \mathbf{Q} \underline{x}_{o}(\tau)+\underline{u}^{T}(\tau) \mathbf{R} \underline{u}(\tau)\right]
$$

Recalling Eq. (2.16) as the linearized metastate-space system which encapsulates the exogenous component into state vector, $\underline{x}_{o},[20]$

$$
f\left(\underline{x}_{o}, \underline{u}, \tau\right)=\underline{\dot{x}}_{o}=\mathbf{A}_{\mathbf{o}} \underline{x}_{o}+\mathbf{B}_{\mathbf{o}} \underline{u}
$$

the Hamiltonian, $\mathbf{H}$, is constructed as

$$
\mathbf{H}\left(\underline{x}_{o}, \underline{u}, \tau\right)=L\left(\underline{x}_{o}, \underline{u}, \tau\right)+\lambda^{T} f\left(\underline{x}_{o}, \underline{u}, \tau\right)
$$


Substituting Eq. (C.4) and Eq. (C.5) into Eq. (C.6) yields the fully expanded Hamiltonian formulation which describes the total minimization problem of performance index in conjunction with the constraint [27],

$$
\mathbf{H}\left(\underline{x}_{o}, \underline{u}, \tau\right)=\frac{1}{2}\left[\underline{x}_{o}^{T}(\tau) \mathbf{Q} \underline{x}_{o}(\tau)+\underline{u}^{T}(\tau) \mathbf{R} \underline{u}(\tau)\right]+\underline{\lambda}^{T}\left(\mathbf{A}_{\mathbf{o}} \underline{x}_{o}+\mathbf{B}_{\mathbf{o}} \underline{u}\right)
$$

where $\underline{\lambda}$ is the vector of Lagrange multipliers or the costate vector. The partial derivative of the Hamiltonian with respect to the costate vector yields the original constraint of the minimization problem in Eq. (C.5) [27].

$$
\frac{\partial \mathbf{H}}{\partial \underline{\lambda}}=\mathbf{A}_{\mathbf{o}} \underline{x}_{o}+\mathbf{B}_{\mathbf{o}} \underline{u}=\underline{\dot{x}}_{o}=f\left(\underline{x}_{o}, \underline{u}, \tau\right)
$$

The partial derivative of the Hamiltonian with respect to the metastate vector, $\underline{x}_{o}$, yields the meta-system costate equation as [27]

$$
\frac{\partial \mathbf{H}}{\partial \underline{x}_{o}}=\frac{\partial L}{\partial \underline{x}_{o}}+\frac{\partial f^{T}}{\partial \underline{x}_{o}} \underline{\lambda}=\mathbf{Q} \underline{x}_{o}+\mathbf{A}_{\mathbf{o}}{ }^{T} \underline{\lambda}=-\underline{\dot{\lambda}}
$$

Finally, the stationarity condition is found by considering the partial derivative of the Hamiltonian with respect to the control action, [27]

$$
\frac{\partial \mathbf{H}}{\partial \underline{u}}=\mathbf{R} \underline{u}+\mathbf{B}_{\mathbf{o}}^{T} \underline{\lambda}=0
$$

Solving Eq. (C.10) for $\underline{u}$, the optimizing control law is found to be [27]

$$
\underline{u}=-\mathbf{R}^{-1} \mathbf{B}_{\mathbf{o}}{ }^{T} \underline{\lambda}
$$

where the costate $\underline{\lambda}$ develops backwards in time as dictated by the governing Eq. (C.9). Substituting the optimal control law into the open loop metastate-space Eq. (C.5), the closed loop metastate-space equation is found as [27]

$$
\underline{\dot{x}}_{o}=\mathbf{A}_{\mathbf{o}} \underline{x}_{o}-\mathbf{B}_{\mathbf{o}} \mathbf{R}^{-1} \mathbf{B}_{\mathbf{o}}^{T} \underline{\lambda}
$$


and develops forward in time. Combining the forward time developing Eq. (C.12) with the backward time developing Eq. (C.9), the Hamiltonian system is formulated [27]

$$
\left[\begin{array}{c}
\frac{\dot{x}_{o}}{\dot{\lambda}}
\end{array}\right]=\left[\begin{array}{ll}
\mathbf{A}_{\mathbf{o}} & -\mathbf{B}_{\mathbf{o}} \mathbf{R}^{-1} \mathbf{B}_{\mathbf{o}}{ }^{T} \\
-\mathbf{Q} & -\mathbf{A}_{\mathbf{o}}{ }^{T}
\end{array}\right]\left[\begin{array}{c}
\underline{x}_{o} \\
\underline{\lambda}
\end{array}\right]
$$

which is characterized by an interesting set of symmetric eigenvalues on the s-plane. The metastate-system closed loop roots exist in the left half stable plane while the roots corresponding to the costate vector offer a direct reflection in the unstable right half plane.

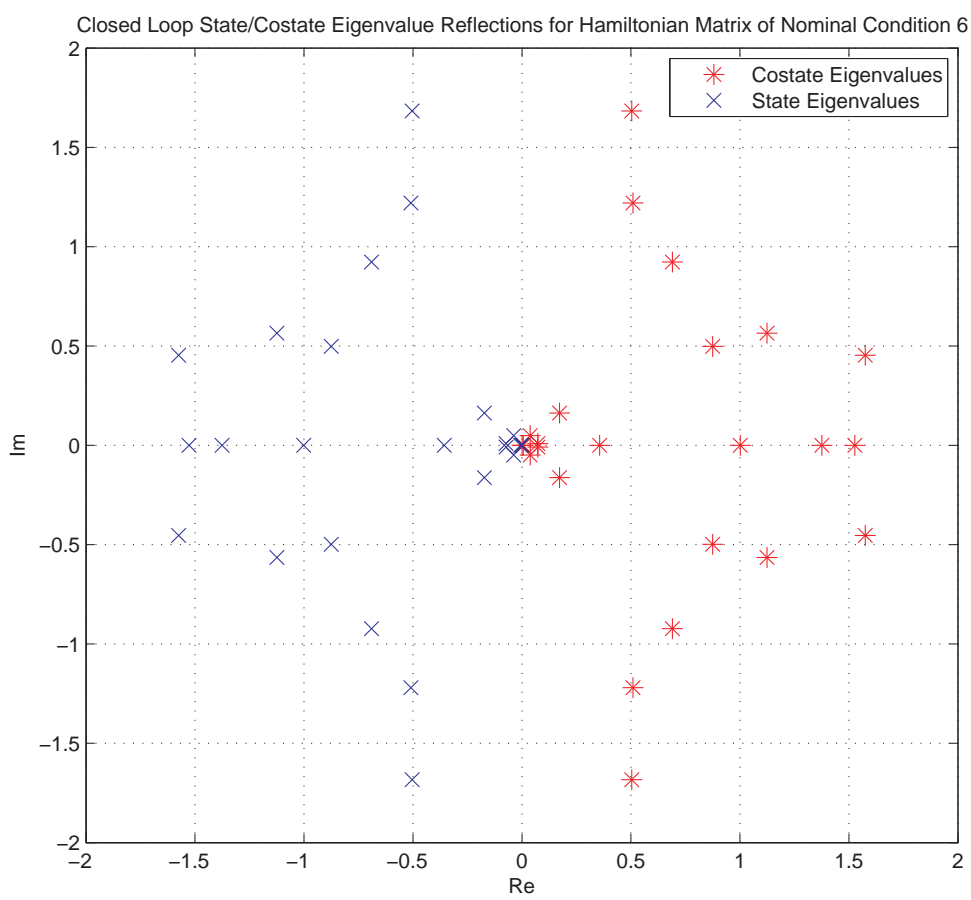

Figure C.1. Closed Loop State/Costate Eigenvalue Reflections for Hamiltonian Matrix of Nominal Condition 6 . 
As a "free-final-state" problem, the boundary condition equates the final costate, $\underline{\lambda}(T)$ to the partial derivative of the function $\phi$ with respect to the final state, $\underline{x}_{o}(T)[27]$.

$$
\frac{\partial \phi}{\partial \underline{x}_{o}(T)}=\underline{\lambda}(T)
$$

Therefore, differentiating Eq. (C.3) with respect to $\underline{x}_{o}(T)$,

$$
\underline{\lambda}(T)=\hat{\mathbf{M}}(T) \underline{x}_{o}(T)
$$

Using the "sweep method" from Bryson and Ho [28], Eq. (C.15) is assumed to hold for the entire time interval (the duration in which the system moves from initial to final state) [27]. Therefore,

$$
\underline{\lambda}(t)=\hat{\mathbf{M}}(t) \underline{x}_{o}(t)
$$

Differentiating with respect to time,

$$
\dot{\dot{\lambda}}=\dot{\hat{\mathbf{M}}} \underline{x}_{o}+\hat{\mathbf{M}} \underline{x}_{o}
$$

Substituting the closed loop metastate-space Eq. (C.12) for $\underline{\dot{x}}_{o}$ as well as utilizing Eq. (C.16), Eq. (C.17) is expanded as

$$
\underline{\dot{\lambda}}=\dot{\hat{\mathbf{M}}} \underline{x}_{o}+\hat{\mathbf{M}}\left(\mathbf{A}_{\mathbf{o}} \underline{x}_{o}-\mathbf{B}_{\mathbf{o}} \mathbf{R}^{-1} \mathbf{B}_{\mathbf{o}}^{T} \hat{\mathbf{M}} \underline{x}_{o}\right)
$$

Recalling the meta-system costate equation in Eq. (C.9), Eq. (C.18) becomes [27]

$$
-\mathbf{Q} \underline{x}_{o}-\mathbf{A}_{\mathbf{o}}^{T} \hat{\mathbf{M}} \underline{x}_{o}=\dot{\hat{\mathbf{M}}} \underline{x}_{o}+\hat{\mathbf{M}}\left(\mathbf{A}_{\mathbf{o}} \underline{x}_{o}-\mathbf{B}_{\mathbf{o}} \mathbf{R}^{-1} \mathbf{B}_{\mathbf{o}}^{T} \hat{\mathbf{M}} \underline{x}_{o}\right)
$$

Rearranging $\dot{\hat{\mathbf{M}}}$ to the left hand side and removing all common $\underline{x}_{o}$ terms, the matrix Ricatti equation is found as [27]

$$
-\dot{\hat{\mathbf{M}}}=\mathbf{A}_{\mathbf{o}}{ }^{T} \hat{\mathbf{M}}+\hat{\mathbf{M}} \mathbf{A}_{\mathbf{o}}-\hat{\mathbf{M}} \mathbf{B}_{\mathbf{o}} \mathbf{R}^{-1} \mathbf{B}_{\mathbf{o}}{ }^{T} \hat{\mathbf{M}}+\mathbf{Q}
$$

Eq. (C.20) is the governing differential equation for the Ricatti kernel matrix, $\hat{\mathbf{M}}$, and develops backwards in time from the final $T$ to initial $t_{o}$. Substituting Eq. (C.16) into 
the control law Eq. (C.11), the time variant control law optimal solution as a function of the Ricatti kernel matrix is found as [27].

$$
\underline{u}(t)=-\mathbf{R}^{-1} \mathbf{B}_{\mathbf{o}}{ }^{T} \hat{\mathbf{M}}(t) \underline{x}_{o}(t)
$$

Therefore, in order to realize the optimal control law, Eq. (C.20) is integrated to find the time variant Ricatti kernel matrix. A typical solution may be observed in Fig. (C.2). Note the negative time axis which reflects the integration begins at the final time, $T$, and solves to the initial $t_{o}$.

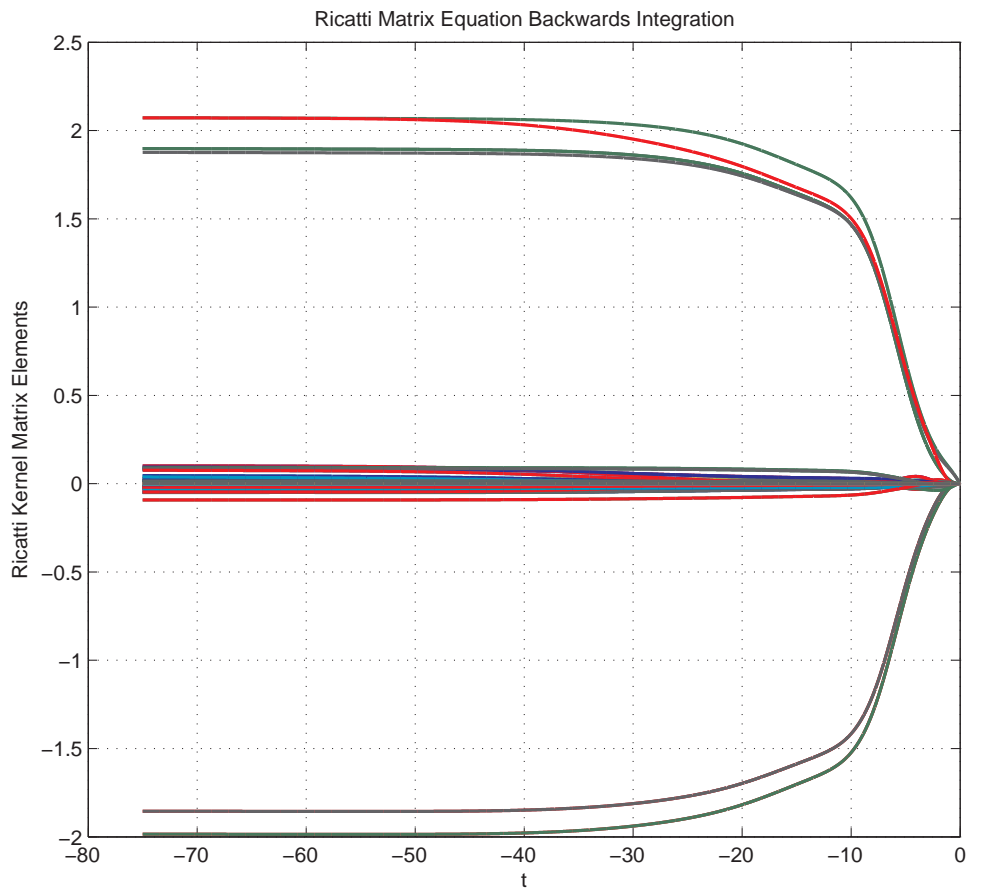

Figure C.2. Ricatti Matrix Equation Backwards Integration for Nominal Condition 6. 
Clearly, the Ricatti kernel matrix approaches a steady state solution in Fig. (C.2) (as $t$ approaches $-\infty$ ), which may be utilized in a suboptimal time invariant control law of the form

$$
\underline{u}(t)=-\mathbf{R}^{-1} \mathbf{B}_{\mathbf{o}}^{T} \overline{\mathbf{M}} \underline{x}_{o}(t)
$$

where $\overline{\mathbf{M}}$ denotes the steady state Ricatti kernel. Note the control law in Eq. (C.22) is considered time invariant due to the constant Kalman gain, $\mathbf{R}^{-1} \mathbf{B}_{\mathbf{o}}{ }^{T} \overline{\mathbf{M}}$. Rather than integrating Eq. (C.20) for prolonged durations to recognize the steady state solution for a suboptimal control law, a direct analytic computation is found using the eigenvalues of the Hamiltonian matrix from Eq. (C.13). Formulating a new matrix, D, consisting of the closed loop eigenvalues from the Hamiltonian matrix,

$$
\mathbf{D}=\left[\begin{array}{ll}
-\mathrm{D}_{\text {eig }} & 0 \\
0 & \mathrm{D}_{\text {eig }}
\end{array}\right]
$$

where $\mathbf{D}_{\text {eig }}$ is a diagonal matrix containing the costate closed loop eigenvalues (i.e. right half plane roots). The corresponding eigenvectors, arranged in order with respect to $\mathbf{D}$, are used to construct a new matrix [27]

$$
\mathrm{W}=\left[\begin{array}{ll}
\mathbf{W}_{11} & \mathbf{W}_{12} \\
\mathbf{W}_{21} & \mathbf{W}_{22}
\end{array}\right]
$$

where $\left[\mathbf{W}_{\mathbf{1 1}}{ }^{T} \mathbf{W}_{\mathbf{2 1}}{ }^{T}\right]^{T}$ are the eigenvectors of the stable closed loop metastate poles. From these matrices, the direct analytic steady state solution to Eq. (C.20) is realized as $[27]$

$$
\overline{\mathbf{M}}=\mathbf{W}_{21} \mathbf{W}_{11}^{-1}
$$

Again, Eq. (C.25) provides the solution to the algebraic Ricatti equation when $\dot{\hat{M}}$ equals zero. Therefore, the suboptimal control law problem is governed by

$$
\mathbf{0}=\mathbf{A}_{\mathbf{o}}{ }^{T} \hat{\mathbf{M}}+\hat{\mathbf{M}} \mathbf{A}_{\mathbf{o}}-\hat{\mathbf{M}} \mathbf{B}_{\mathbf{o}} \mathbf{R}^{-1} \mathbf{B}_{\mathbf{o}}{ }^{T} \hat{\mathbf{M}}+\mathbf{Q}
$$


Next, this equation is partitioned in accordance with the metasystem formulation in order to understand the Friedland control law utilized on the feedback states and feed-forward exogenous components. Recalling the Friedland procedure provided in detail in Chapter 2 which encapsulates the exogenous components into the state-space matrices as

$$
\mathbf{A}_{\mathbf{o}}=\left[\begin{array}{c|c}
\mathbf{A} & \mathbf{E} \\
\hline \mathbf{0} & \mathbf{A}_{\mathbf{T}_{\mathbf{C L}}}
\end{array}\right] \text { and } \mathbf{B}_{\mathbf{o}}=\left[\begin{array}{c}
\mathbf{B} \\
\hline \mathbf{0}
\end{array}\right] \text {, }
$$

and the performance index matrix of

$$
\mathrm{Q}=\left[\begin{array}{c|c}
\mathrm{Q}_{x} & \mathbf{0} \\
\hline \mathbf{0} & \mathbf{0}
\end{array}\right]
$$

Eq. (C.20) and Eq. (C.26) expand as the following

$$
\begin{aligned}
& {\left[\begin{array}{cc}
-\dot{\hat{\mathbf{M}}}_{\mathbf{1}} & -\dot{\hat{\mathbf{M}}}_{\mathbf{2}} \\
-\dot{\hat{\mathbf{M}}}_{\mathbf{2}}{ }^{T} & -\dot{\hat{\mathbf{M}}}_{\mathbf{3}}
\end{array}\right]=\left[\begin{array}{ll}
\mathbf{A}^{T} & \mathbf{0} \\
\mathbf{E}^{T} & \mathbf{A}_{\mathbf{T}_{\mathbf{C L}}}{ }^{T}
\end{array}\right]\left[\begin{array}{ll}
\hat{\mathbf{M}}_{\mathbf{1}} & \hat{\mathbf{M}}_{\mathbf{2}} \\
\hat{\mathbf{M}}_{\mathbf{2}}^{T} & \hat{\mathbf{M}}_{\mathbf{3}}
\end{array}\right]+\left[\begin{array}{ll}
\hat{\mathbf{M}}_{\mathbf{1}} & \hat{\mathbf{M}}_{\mathbf{2}} \\
\hat{\mathbf{M}}_{2}^{T} & \hat{\mathbf{M}}_{\mathbf{3}}
\end{array}\right]\left[\begin{array}{ll}
\mathbf{A} & \mathbf{E} \\
\mathbf{0} & \mathbf{A}_{\mathbf{T}_{\mathbf{C L}}}
\end{array}\right]} \\
& -\left[\begin{array}{cc}
\hat{\mathbf{M}}_{1} & \hat{\mathbf{M}}_{2} \\
\hat{\mathbf{M}}_{2}^{T} & \hat{\mathbf{M}}_{3}
\end{array}\right]\left[\begin{array}{l}
\mathbf{B} \\
0
\end{array}\right] \mathbf{R}^{-1}\left[\mathbf{B}^{T} \mathbf{0}\right]\left[\begin{array}{ll}
\hat{\mathbf{M}}_{1} & \hat{\mathbf{M}}_{2} \\
\hat{\mathbf{M}}_{2}^{T} & \hat{\mathbf{M}}_{3}
\end{array}\right] \\
& +\left[\begin{array}{cc}
\mathrm{Q}_{x} & 0 \\
0 & 0
\end{array}\right]=\left[\begin{array}{ll}
0 & 0 \\
0 & 0
\end{array}\right]
\end{aligned}
$$

Recognizing that the suboptimal control law in Eq. (C.22) creates a closed loop action impacting the receiver state rate alone, only the top row of Eq. (C.27) is solved for $\hat{\mathbf{M}}_{\mathbf{1}}$ and $\hat{\mathbf{M}}_{2}$ (which are independent of $\hat{\mathbf{M}}_{3}$ ). Extraction of these two equations yields

$$
\begin{gathered}
-\dot{\hat{M}}_{1}=\hat{M}_{1} A+A^{T} \hat{M}_{1}-\hat{M}_{1} B R^{-1} B^{T} \hat{M}_{1}+Q_{x}=0 \\
-\dot{\hat{M}}_{2}=\hat{M}_{1} \mathbf{E}+\hat{M}_{2} A_{T_{C L}}+\left(A^{T}-\hat{M}_{1} B R^{-1} B^{T}\right) \hat{M}_{2}=0
\end{gathered}
$$

whose steady state solutions, $\overline{\mathbf{M}}_{1}$ and $\overline{\mathbf{M}}_{2}$, govern the receiver control law.

$$
\underline{u}=-\mathbf{R}^{-1} \mathbf{B}^{\mathbf{T}} \overline{\mathbf{M}}_{1} \underline{x}-\mathbf{R}^{-1} \mathbf{B}^{\mathbf{T}} \overline{\mathbf{M}}_{2} \underline{x}_{T_{A}}
$$


APPENDIX D

TRANSFORMATION OF RECEIVER DISTURBANCE STATE-SPACE MATRIX 
This appendix provides the elements of the $\mathbf{H}(\mathbf{t})$ matrix which quantifies disturbance in the receiver linearized state-space system, as described in Chapter 2 in Eq. (2.12). Also provided are the elements of $\boldsymbol{\Upsilon}(\mathbf{t})$, which is used as the linear transformation from the tanker state vector to the receiver disturbance vector, as presented in Chapter 4 .

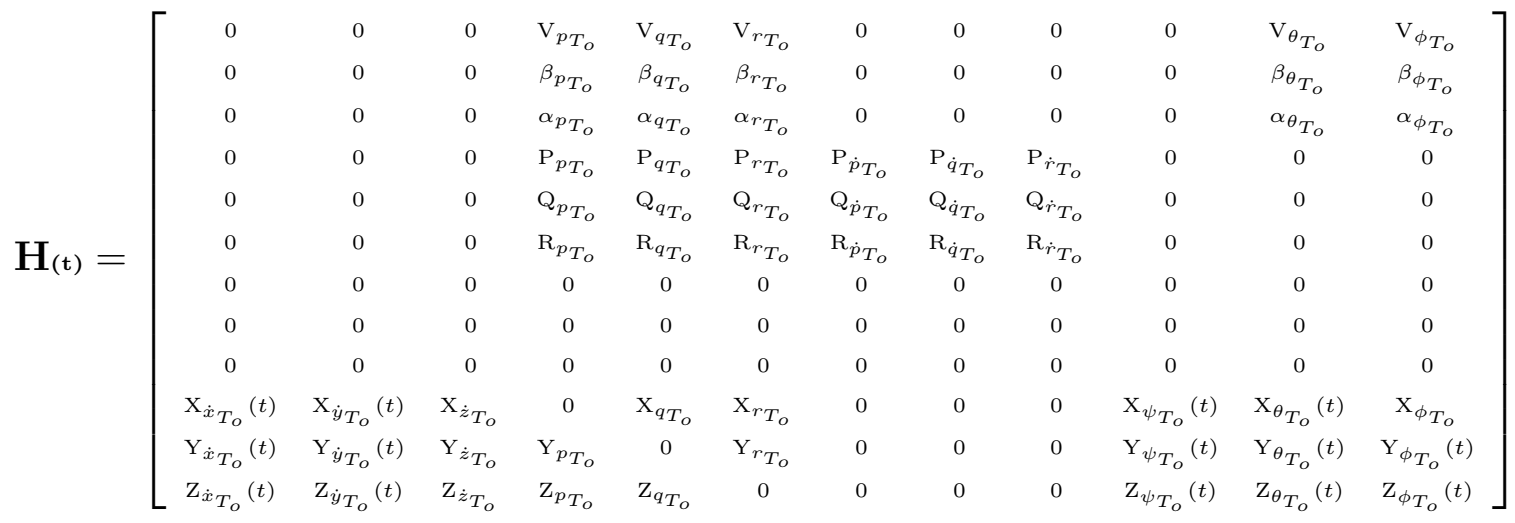

$$
\begin{aligned}
& \mathrm{V}_{p_{T_{o}}}=0 \\
& \mathrm{~V}_{q_{T_{o}}}=0 \\
& \mathrm{~V}_{r_{T_{o}}}=0 \\
& \mathrm{~V}_{\theta_{T_{o}}}=-g m_{R} \cos \theta_{T}\left\{[\cos \beta \sin \alpha(\sin \phi \sin \psi+\cos \phi \cos \psi \sin \theta)] / m_{R}-[\sin \beta(\cos \phi \sin \psi-\cos \psi \sin \phi \sin \theta)] / m_{R}\right. \\
& \left.+(\cos \alpha \cos \beta \cos \psi \cos \theta) / m_{R}\right\}-g m_{R} \cos \phi_{T} \sin \theta_{T}\left[(\sin \beta \cos \theta \sin \phi) / m_{R}-(\cos \alpha \cos \beta \sin \theta) / m_{R}\right. \\
& \left.+(\cos \beta \cos \phi \sin \alpha \cos \theta) / m_{R}\right]-g m_{R} \sin \phi_{T} \sin \theta_{T}\left\{[\sin \beta(\cos \phi \cos \psi+\sin \phi \sin \psi \sin \theta)] / m_{R}\right. \\
& \left.-[\cos \beta \sin \alpha(\cos \psi \sin \phi-\cos \phi \sin \psi \sin \theta)] / m_{R}+(\cos \alpha \cos \beta \cos \theta \sin \psi) / m_{R}\right\}\left.\right|_{o} \\
& \mathrm{~V}_{\phi_{T_{o}}}=g m_{R} \cos \phi_{T} \cos \theta_{T}\left\{[\sin \beta(\cos \phi \cos \psi+\sin \phi \sin \psi \sin \theta)] / m_{R}-[\cos \beta \sin \alpha(\cos \psi \sin \phi\right. \\
& \left.-\cos \phi \sin \psi \sin \theta)] / m_{R}+(\cos \alpha \cos \beta \cos \theta \sin \psi) / m_{R}\right\}-g m_{R} \cos \theta_{T} \sin \phi_{T}\left[(\sin \beta \cos \theta \sin \phi) / m_{R}\right. \\
& \left.-(\cos \alpha \cos \beta \sin \theta) / m_{R}+(\cos \beta \cos \phi \sin \alpha \cos \theta) / m_{R}\right]\left.\right|_{o} \\
& \beta_{p_{T_{o}}}=\cos \psi \sin \alpha \cos \theta-\cos \alpha \sin \phi \sin \psi-\left.\cos \alpha \cos \phi \cos \psi \sin \theta\right|_{o} \\
& \beta_{q_{T_{o}}}=\cos \alpha \cos \psi \sin \phi+\sin \alpha \cos \theta \sin \psi-\left.\cos \alpha \cos \phi \sin \psi \sin \theta\right|_{o} \\
& \beta_{r_{T_{o}}}=-\sin \alpha \sin \theta-\left.\cos \alpha \cos \phi \cos \theta\right|_{o} \\
& \beta_{\theta_{T_{o}}}=g m_{R} \cos \theta_{T}\left\{[\cos \beta(\cos \phi \sin \psi-\cos \psi \sin \phi \sin \theta)] /\left(V m_{R}\right)+[\sin \alpha \sin \beta(\sin \phi \sin \psi\right. \\
& \left.+\cos \phi \cos \psi \sin \theta)] /\left(V m_{R}\right)+(\cos \alpha \cos \psi \sin \beta \cos \theta) /\left(V m_{R}\right)\right\} \\
& -g m_{R} \cos \phi_{T} \sin \theta_{T}\left[(\cos \beta \cos \theta \sin \phi) /\left(V m_{R}\right)+(\cos \alpha \sin \beta \sin \theta) /\left(V m_{R}\right)\right. \\
& \left.-(\cos \phi \sin \alpha \sin \beta \cos \theta) /\left(V m_{R}\right)\right]-g m_{R} \sin \phi_{T} \sin \theta_{T}\{[\cos \beta(\cos \phi \cos \psi
\end{aligned}
$$




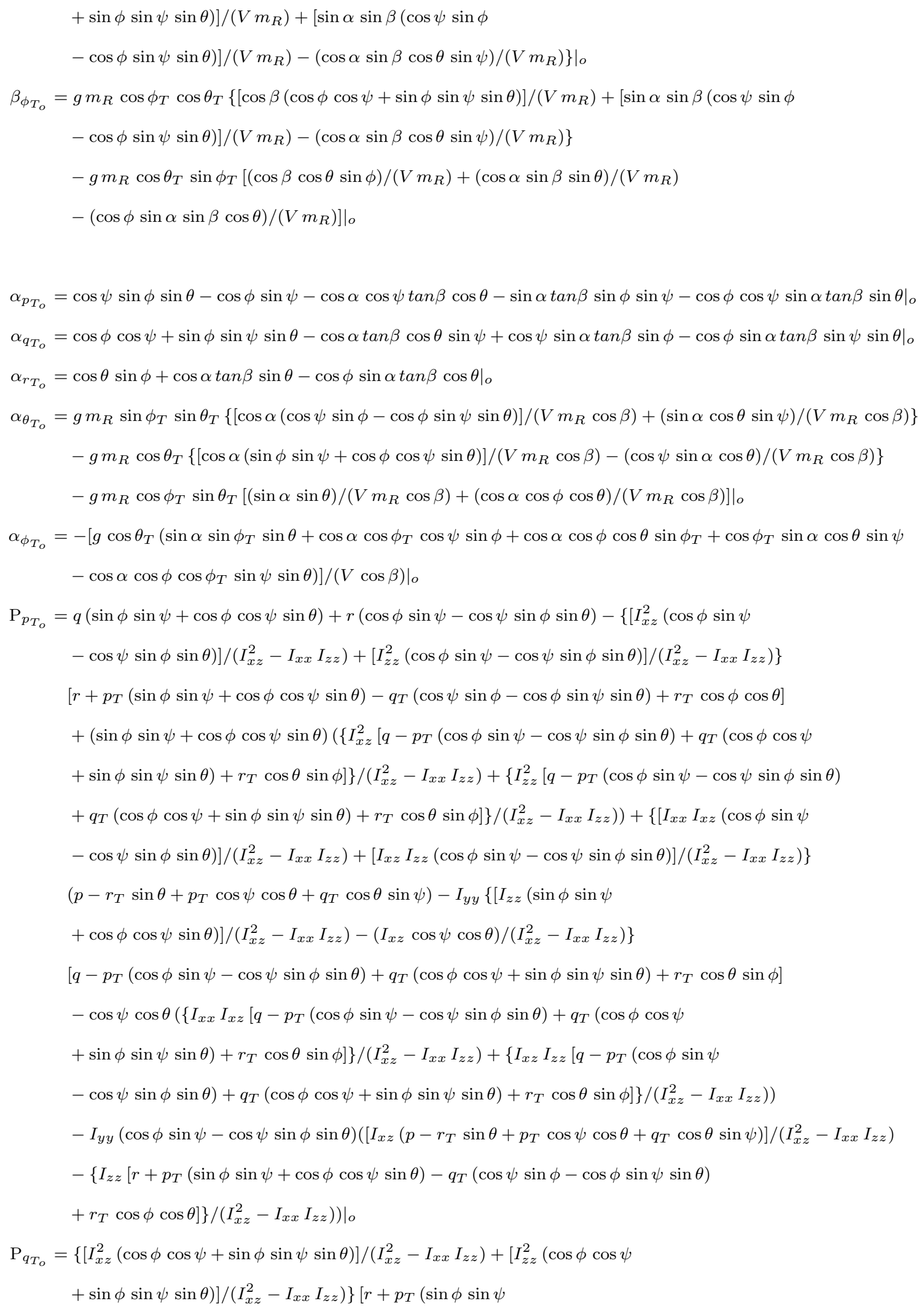


$+\cos \phi \cos \psi \sin \theta)-q_{T}(\cos \psi \sin \phi-\cos \phi \sin \psi \sin \theta)$

$\left.+r_{T} \cos \phi \cos \theta\right]-r(\cos \phi \cos \psi+\sin \phi \sin \psi \sin \theta)$

$-q(\cos \psi \sin \phi-\cos \phi \sin \psi \sin \theta)-(\cos \psi \sin \phi$

$-\cos \phi \sin \psi \sin \theta)\left(\left\{I_{x z}^{2}\left[q-p_{T}(\cos \phi \sin \psi-\cos \psi \sin \phi \sin \theta)\right.\right.\right.$

$\left.\left.+q_{T}(\cos \phi \cos \psi+\sin \phi \sin \psi \sin \theta)+r_{T} \cos \theta \sin \phi\right]\right\} /\left(I_{x z}^{2}-I_{x x} I_{z z}\right)$

$+\left\{I_{z z}^{2}\left[q-p_{T}(\cos \phi \sin \psi-\cos \psi \sin \phi \sin \theta)+q_{T}(\cos \phi \cos \psi\right.\right.$

$\left.\left.\left.+\sin \phi \sin \psi \sin \theta)+r_{T} \cos \theta \sin \phi\right]\right\} /\left(I_{x z}^{2}-I_{x x} I_{z z}\right)\right)-\left\{\left[I_{x x} I_{x z}(\cos \phi \cos \psi\right.\right.$

$+\sin \phi \sin \psi \sin \theta)] /\left(I_{x z}^{2}-I_{x x} I_{z z}\right)+\left[I_{x z} I_{z z}(\cos \phi \cos \psi\right.$

$\left.+\sin \phi \sin \psi \sin \theta)] /\left(I_{x z}^{2}-I_{x x} I_{z z}\right)\right\}\left(p-r_{T} \sin \theta+p_{T} \cos \psi \cos \theta+q_{T} \cos \theta \sin \psi\right)$

$+I_{y y}\left\{\left[I_{z z}(\cos \psi \sin \phi-\cos \phi \sin \psi \sin \theta)\right] /\left(I_{x z}^{2}-I_{x x} I_{z z}\right)\right.$

$\left.+\left(I_{x z} \cos \theta \sin \psi\right) /\left(I_{x z}^{2}-I_{x x} I_{z z}\right)\right\}\left[q-p_{T}(\cos \phi \sin \psi-\cos \psi \sin \phi \sin \theta)\right.$

$\left.+q_{T}(\cos \phi \cos \psi+\sin \phi \sin \psi \sin \theta)+r_{T} \cos \theta \sin \phi\right]$

$-\cos \theta \sin \psi\left(\left\{I_{x x} I_{x z}\left[q-p_{T}(\cos \phi \sin \psi-\cos \psi \sin \phi \sin \theta)\right.\right.\right.$

$\left.\left.+q_{T}(\cos \phi \cos \psi+\sin \phi \sin \psi \sin \theta)+r_{T} \cos \theta \sin \phi\right]\right\} /\left(I_{x z}^{2}-I_{x x} I_{z z}\right)$

$+\left\{I_{x z} I_{z z}\left[q-p_{T}(\cos \phi \sin \psi-\cos \psi \sin \phi \sin \theta)\right.\right.$

$\left.\left.\left.+q_{T}(\cos \phi \cos \psi+\sin \phi \sin \psi \sin \theta)+r_{T} \cos \theta \sin \phi\right]\right\} /\left(I_{x z}^{2}-I_{x x} I_{z z}\right)\right)$

$+I_{y y}(\cos \phi \cos \psi+\sin \phi \sin \psi \sin \theta)\left(\left[I_{x z}\left(p-r_{T} \sin \theta\right.\right.\right.$

$\left.\left.+p_{T} \cos \psi \cos \theta+q_{T} \cos \theta \sin \psi\right)\right] /\left(I_{x z}^{2}-I_{x x} I_{z z}\right)-\left\{I_{z z}\left[r+p_{T}(\sin \phi \sin \psi\right.\right.$

$+\cos \phi \cos \psi \sin \theta)-q_{T}(\cos \psi \sin \phi-\cos \phi \sin \psi \sin \theta)$

$\left.\left.\left.+r_{T} \cos \phi \cos \theta\right]\right\} /\left(I_{x z}^{2}-I_{x x} I_{z z}\right)\right)\left.\right|_{o}$

$\mathrm{P}_{r_{T_{o}}}=\sin \theta\left(\left\{I_{x x} I_{x z}\left[q-p_{T}(\cos \phi \sin \psi-\cos \psi \sin \phi \sin \theta)+q_{T}(\cos \phi \cos \psi+\sin \phi \sin \psi \sin \theta)\right.\right.\right.$

$\left.\left.+r_{T} \cos \theta \sin \phi\right]\right\} /\left(I_{x z}^{2}-I_{x x} I_{z z}\right)+\left\{I_{x z} I_{z z}\left[q-p_{T}(\cos \phi \sin \psi-\cos \psi \sin \phi \sin \theta)+q_{T}(\cos \phi \cos \psi\right.\right.$

$\left.\left.\left.+\sin \phi \sin \psi \sin \theta)+r_{T} \cos \theta \sin \phi\right]\right\} /\left(I_{x z}^{2}-I_{x x} I_{z z}\right)\right)-\left[\left(I_{x x} I_{x z} \cos \theta \sin \phi\right) /\left(I_{x z}^{2}-I_{x x} I_{z z}\right)\right.$

$\left.+\left(I_{x z} I_{z z} \cos \theta \sin \phi\right) /\left(I_{x z}^{2}-I_{x x} I_{z z}\right)\right]\left(p-r_{T} \sin \theta+p_{T} \cos \psi \cos \theta+q_{T} \cos \theta \sin \psi\right)$

$+\left[\left(I_{x z}^{2} \cos \theta \sin \phi\right) /\left(I_{x z}^{2}-I_{x x} I_{z z}\right)+\left(I_{z z}^{2} \cos \theta \sin \phi\right) /\left(I_{x z}^{2}-I_{x x} I_{z z}\right)\right]\left[r+p_{T}(\sin \phi \sin \psi\right.$

$\left.+\cos \phi \cos \psi \sin \theta)-q_{T}(\cos \psi \sin \phi-\cos \phi \sin \psi \sin \theta)+r_{T} \cos \phi \cos \theta\right]$

$+\cos \phi \cos \theta\left(\left\{I_{x z}^{2}\left[q-p_{T}(\cos \phi \sin \psi-\cos \psi \sin \phi \sin \theta)\right.\right.\right.$

$\left.\left.+q_{T}(\cos \phi \cos \psi+\sin \phi \sin \psi \sin \theta)+r_{T} \cos \theta \sin \phi\right]\right\} /\left(I_{x z}^{2}-I_{x x} I_{z z}\right)$

$+\left\{I_{z z}^{2}\left[q-p_{T}(\cos \phi \sin \psi-\cos \psi \sin \phi \sin \theta)+q_{T}(\cos \phi \cos \psi\right.\right.$

$\left.\left.\left.+\sin \phi \sin \psi \sin \theta)+r_{T} \cos \theta \sin \phi\right]\right\} /\left(I_{x z}^{2}-I_{x x} I_{z z}\right)\right)+q \cos \phi \cos \theta$

$-I_{y y}\left[\left(I_{x z} \sin \theta\right) /\left(I_{x z}^{2}-I_{x x} I_{z z}\right)+\left(I_{z z} \cos \phi \cos \theta\right) /\left(I_{x z}^{2}-I_{x x} I_{z z}\right)\right]\left[q-p_{T}(\cos \phi \sin \psi\right.$

$-\cos \psi \sin \phi \sin \theta)+q_{T}(\cos \phi \cos \psi+\sin \phi \sin \psi \sin \theta)$

$\left.+r_{T} \cos \theta \sin \phi\right]-r \cos \theta \sin \phi+I_{y y} \cos \theta \sin \phi\left(\left[I_{x z}\right.\right.$ 


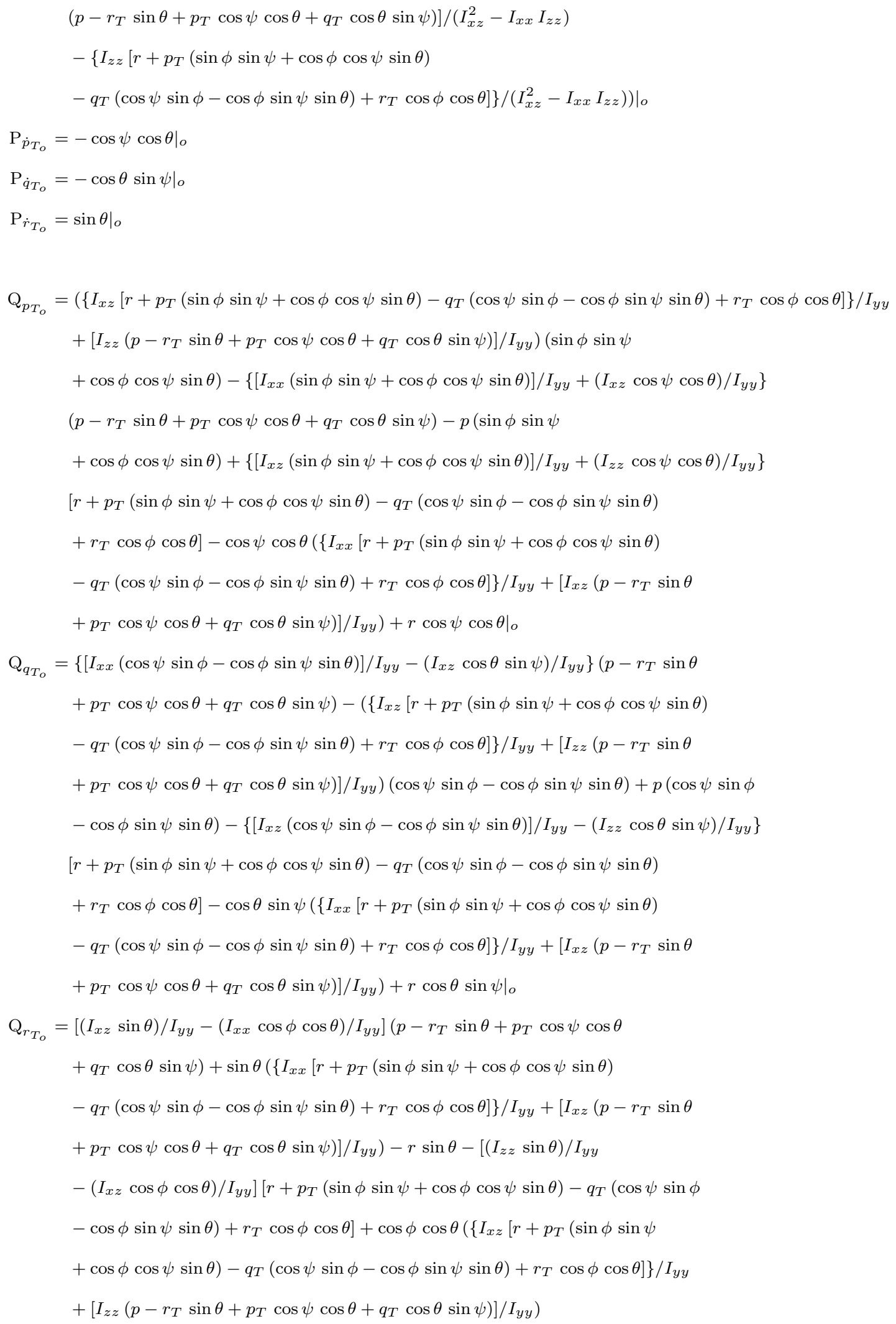




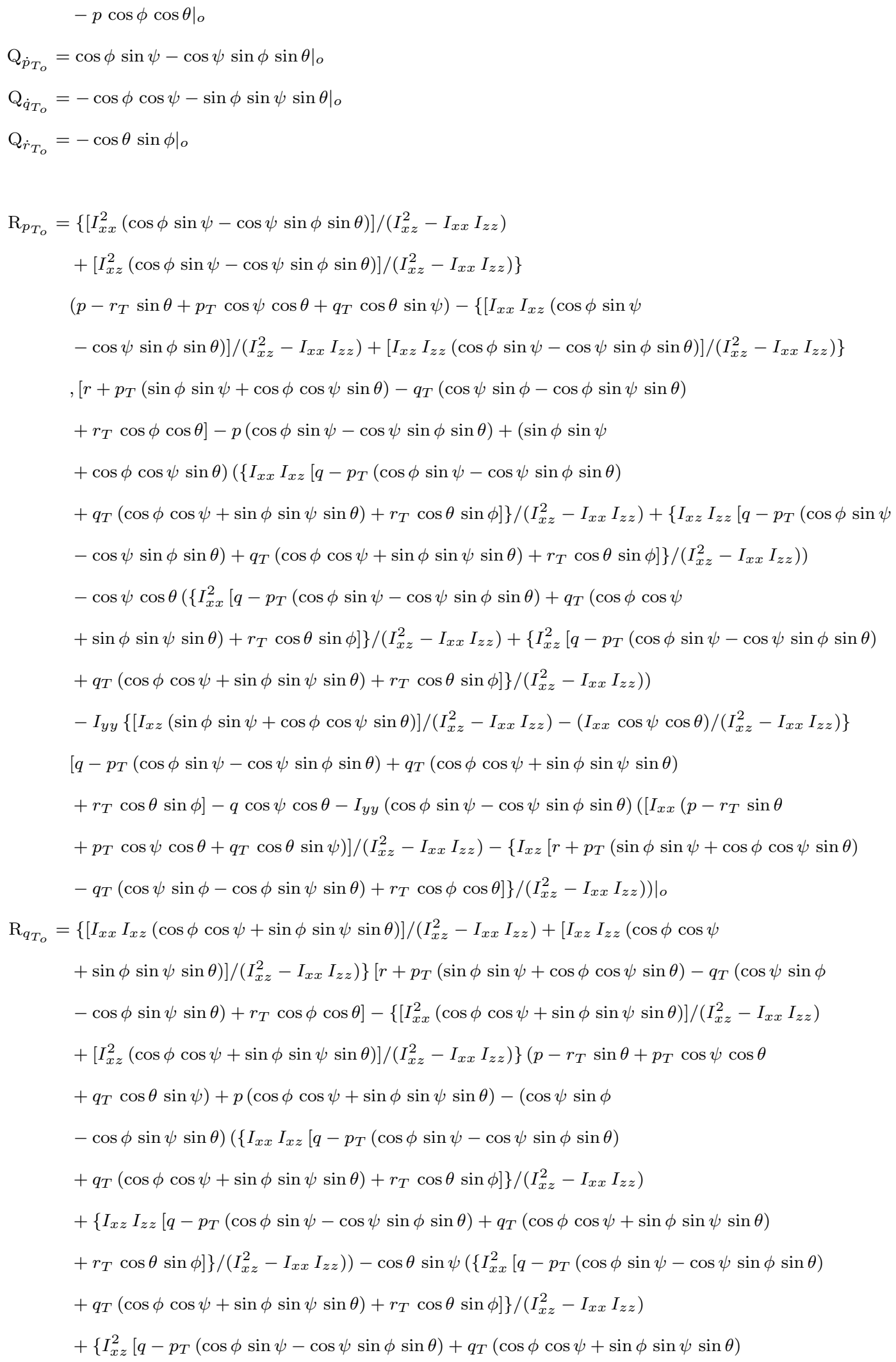




$$
\begin{aligned}
& \left.\left.\left.+r_{T} \cos \theta \sin \phi\right]\right\} /\left(I_{x z}^{2}-I_{x x} I_{z z}\right)\right)+I_{y y}\left\{\left[I_{x z}(\cos \psi \sin \phi-\cos \phi \sin \psi \sin \theta)\right] /\left(I_{x z}^{2}-I_{x x} I_{z z}\right)\right. \\
& \left.+\left(I_{x x} \cos \theta \sin \psi\right) /\left(I_{x z}^{2}-I_{x x} I_{z z}\right)\right\}\left[q-p_{T}(\cos \phi \sin \psi-\cos \psi \sin \phi \sin \theta)+q_{T}(\cos \phi \cos \psi\right. \\
& \left.+\sin \phi \sin \psi \sin \theta)+r_{T} \cos \theta \sin \phi\right]-q \cos \theta \sin \psi+I_{y y}(\cos \phi \cos \psi \\
& +\sin \phi \sin \psi \sin \theta)\left(\left[I_{x x}\left(p-r_{T} \sin \theta+p_{T} \cos \psi \cos \theta+q_{T} \cos \theta \sin \psi\right)\right] /\left(I_{x z}^{2}-I_{x x} I_{z z}\right)\right. \\
& -\left\{I _ { x z } \left[r+p_{T}(\sin \phi \sin \psi+\cos \phi \cos \psi \sin \theta)-q_{T}(\cos \psi \sin \phi-\cos \phi \sin \psi \sin \theta)\right.\right. \\
& \left.\left.\left.+r_{T} \cos \phi \cos \theta\right]\right\} /\left(I_{x z}^{2}-I_{x x} I_{z z}\right)\right)\left.\right|_{o} \\
& \mathrm{R}_{r_{T_{o}}}=\sin \theta\left(\left\{I _ { x x } ^ { 2 } \left[q-p_{T}(\cos \phi \sin \psi-\cos \psi \sin \phi \sin \theta)+q_{T}(\cos \phi \cos \psi\right.\right.\right. \\
& \left.\left.+\sin \phi \sin \psi \sin \theta)+r_{T} \cos \theta \sin \phi\right]\right\} /\left(I_{x z}^{2}-I_{x x} I_{z z}\right)+\left\{I _ { x z } ^ { 2 } \left[q-p_{T}(\cos \phi \sin \psi-\cos \psi \sin \phi \sin \theta)\right.\right. \\
& \left.\left.\left.+q_{T}(\cos \phi \cos \psi+\sin \phi \sin \psi \sin \theta)+r_{T} \cos \theta \sin \phi\right]\right\} /\left(I_{x z}^{2}-I_{x x} I_{z z}\right)\right) \\
& -\left[\left(I_{x x}^{2} \cos \theta \sin \phi\right) /\left(I_{x z}^{2}-I_{x x} I_{z z}\right)+\left(I_{x z}^{2} \cos \theta \sin \phi\right) /\left(I_{x z}^{2}-I_{x x} I_{z z}\right)\right]\left(p-r_{T} \sin \theta\right. \\
& \left.+p_{T} \cos \psi \cos \theta+q_{T} \cos \theta \sin \psi\right)+\left[\left(I_{x x} I_{x z} \cos \theta \sin \phi\right) /\left(I_{x z}^{2}-I_{x x} I_{z z}\right)\right. \\
& \left.+\left(I_{x z} I_{z z} \cos \theta \sin \phi\right) /\left(I_{x z}^{2}-I_{x x} I_{z z}\right)\right]\left[r+p_{T}(\sin \phi \sin \psi+\cos \phi \cos \psi \sin \theta)-q_{T}(\cos \psi \sin \phi\right. \\
& \left.-\cos \phi \sin \psi \sin \theta)+r_{T} \cos \phi \cos \theta\right]+q \sin \theta+p \cos \theta \sin \phi \\
& -I_{y y}\left[\left(I_{x x} \sin \theta\right) /\left(I_{x z}^{2}-I_{x x} I_{z z}\right)+\left(I_{x z} \cos \phi \cos \theta\right) /\left(I_{x z}^{2}-I_{x x} I_{z z}\right)\right]\left[q-p_{T}(\cos \phi \sin \psi\right. \\
& -\cos \psi \sin \phi \sin \theta)+q_{T}(\cos \phi \cos \psi+\sin \phi \sin \psi \sin \theta) \\
& \left.+r_{T} \cos \theta \sin \phi\right]+\cos \phi \cos \theta\left(\left\{I _ { x x } I _ { x z } \left[q-p_{T}(\cos \phi \sin \psi-\cos \psi \sin \phi \sin \theta)\right.\right.\right. \\
& \left.\left.+q_{T}(\cos \phi \cos \psi+\sin \phi \sin \psi \sin \theta)+r_{T} \cos \theta \sin \phi\right]\right\} /\left(I_{x z}^{2}-I_{x x} I_{z z}\right) \\
& +\left\{I _ { x z } I _ { z z } \left[q-p_{T}(\cos \phi \sin \psi-\cos \psi \sin \phi \sin \theta)+q_{T}(\cos \phi \cos \psi\right.\right. \\
& \left.\left.\left.+\sin \phi \sin \psi \sin \theta)+r_{T} \cos \theta \sin \phi\right]\right\} /\left(I_{x z}^{2}-I_{x x} I_{z z}\right)\right)+I_{y y} \cos \theta \sin \phi\left(\left[I _ { x x } \left(p-r_{T} \sin \theta\right.\right.\right. \\
& \left.\left.+p_{T} \cos \psi \cos \theta+q_{T} \cos \theta \sin \psi\right)\right] /\left(I_{x z}^{2}-I_{x x} I_{z z}\right)-\left\{I _ { x z } \left[r+p_{T}(\sin \phi \sin \psi+\cos \phi \cos \psi \sin \theta)\right.\right. \\
& \left.\left.\left.-q_{T}(\cos \psi \sin \phi-\cos \phi \sin \psi \sin \theta)+r_{T} \cos \phi \cos \theta\right]\right\} /\left(I_{x z}^{2}-I_{x x} I_{z z}\right)\right)\left.\right|_{o} \\
& \mathrm{R}_{\dot{p}_{T_{o}}}=-\sin \phi \sin \psi-\left.\cos \phi \cos \psi \sin \theta\right|_{o} \\
& \mathrm{R}_{\dot{q}_{T_{o}}}=\cos \psi \sin \phi-\left.\cos \phi \sin \psi \sin \theta\right|_{o} \\
& \mathrm{R}_{\dot{r}_{T_{o}}}=-\left.\cos \phi \cos \theta\right|_{o} \\
& \mathrm{X}_{\dot{x}_{T_{o}}}(t)=-\left.\cos \psi_{T} \cos \theta_{T}\right|_{o} \\
& \mathrm{X}_{\dot{y}_{T_{o}}}(t)=-\left.\cos \theta_{T} \sin \psi_{T}\right|_{o} \\
& \mathrm{X}_{\dot{z}_{T_{o}}}=\left.\sin \theta_{T}\right|_{o} \\
& \mathrm{X}_{q_{T_{o}}}=-\left.z\right|_{o} \\
& \mathrm{X}_{r_{T_{o}}}=\left.y\right|_{o} \\
& \mathrm{X}_{\psi_{T_{o}}}(t)=-\left.\cos \theta_{T}\left(\dot{y}_{T} \cos \psi_{T}-\dot{x}_{T} \sin \psi_{T}\right)\right|_{o} \\
& \mathrm{X}_{\theta_{T_{o}}}(t)=\dot{z}_{T} \cos \theta_{T}+\dot{x}_{T} \cos \psi_{T} \sin \theta_{T}+\left.\dot{y}_{T} \sin \psi_{T} \sin \theta_{T}\right|_{o} \\
& \mathrm{X}_{\phi_{T_{o}}}=0
\end{aligned}
$$




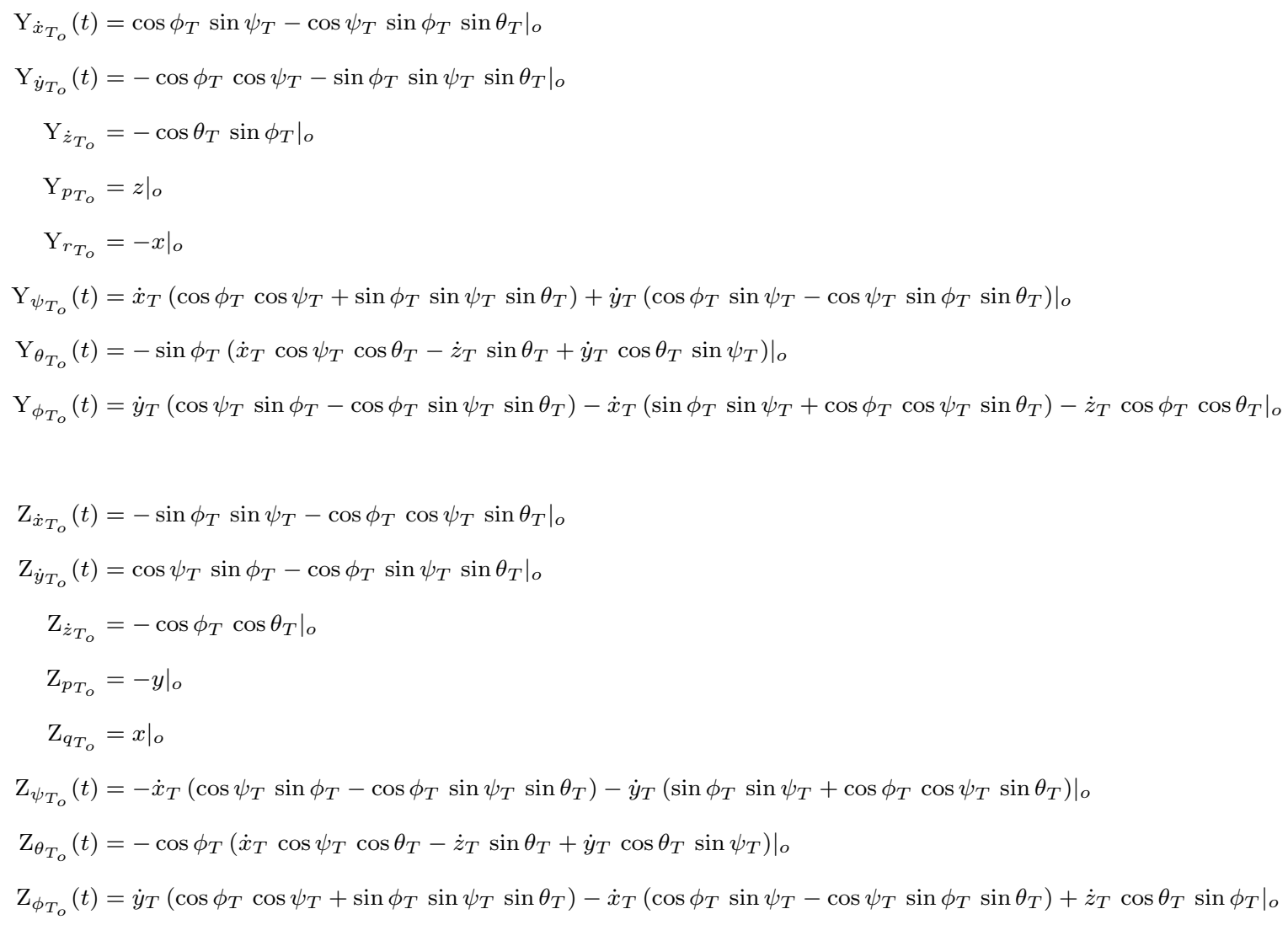

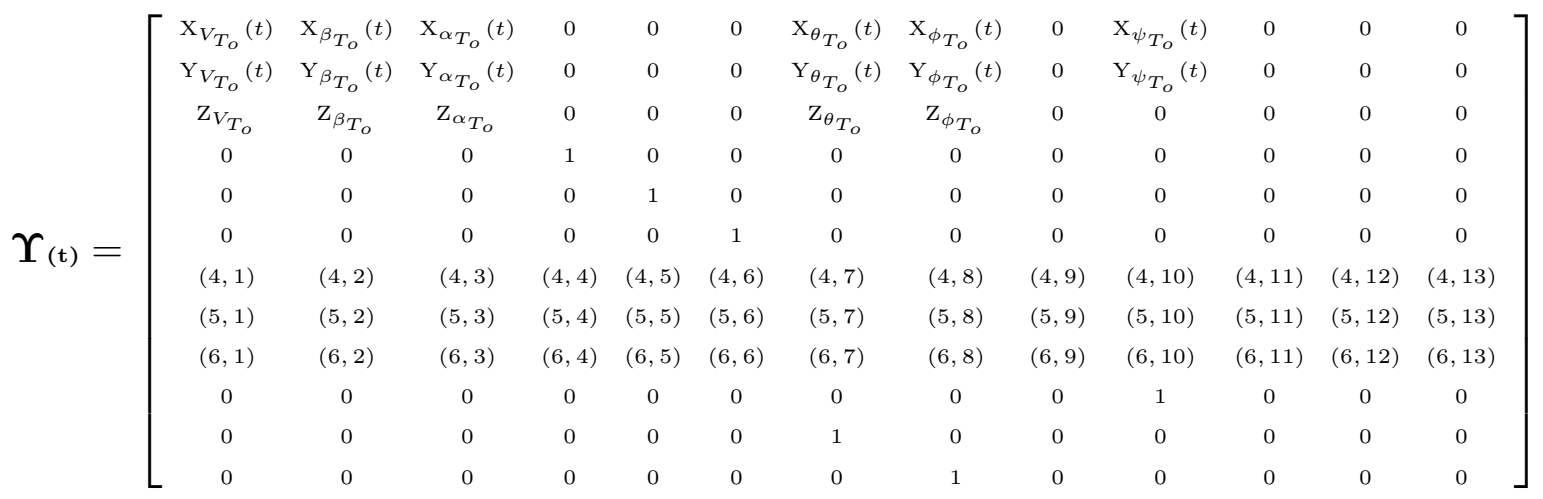

$\mathrm{X}_{V_{T_{o}}}(t)=\cos \beta_{T} \sin \alpha_{T}\left(\sin \phi_{T} \sin \psi_{T}+\cos \phi_{T} \cos \psi_{T} \sin \theta_{T}\right)-\sin \beta_{T}\left(\cos \phi_{T} \sin \psi_{T}-\cos \psi_{T} \sin \phi_{T} \sin \theta_{T}\right)$ $+\left.\cos \alpha_{T} \cos \beta_{T} \cos \psi_{T} \cos \theta_{T}\right|_{o}$ 
$\mathrm{X}_{\beta_{T_{o}}}(t)=-V_{T} \cos \beta_{T}\left(\cos \phi_{T} \sin \psi_{T}-\cos \psi_{T} \sin \phi_{T} \sin \theta_{T}\right)-V_{T} \sin \alpha_{T} \sin \beta_{T}\left(\sin \phi_{T} \sin \psi_{T}\right.$ $\left.+\cos \phi_{T} \cos \psi_{T} \sin \theta_{T}\right)-\left.V_{T} \cos \alpha_{T} \cos \psi_{T} \sin \beta_{T} \cos \theta_{T}\right|_{o}$

$\mathrm{X}_{\alpha_{T_{o}}}(t)=V_{T} \cos \alpha_{T} \cos \beta_{T}\left(\sin \phi_{T} \sin \psi_{T}+\cos \phi_{T} \cos \psi_{T} \sin \theta_{T}\right)-\left.V_{T} \cos \beta_{T} \cos \psi_{T} \sin \alpha_{T} \cos \theta_{T}\right|_{o}$

$\mathrm{X}_{\theta_{T_{o}}}(t)=\left.V_{T} \cos \psi_{T}\left(\sin \beta_{T} \cos \theta_{T} \sin \phi_{T}-\cos \alpha_{T} \cos \beta_{T} \sin \theta_{T}+\cos \beta_{T} \cos \phi_{T} \sin \alpha_{T} \cos \theta_{T}\right)\right|_{o}$

$\mathrm{X}_{\phi_{T_{o}}}(t)=V_{T} \sin \beta_{T}\left(\sin \phi_{T} \sin \psi_{T}+\cos \phi_{T} \cos \psi_{T} \sin \theta_{T}\right)+V_{T} \cos \beta_{T} \sin \alpha_{T}\left(\cos \phi_{T} \sin \psi_{T}\right.$ $\left.-\cos \psi_{T} \sin \phi_{T} \sin \theta_{T}\right)\left.\right|_{o}$

$\mathrm{X}_{\psi_{T_{o}}}(t)=V_{T} \cos \beta_{T} \sin \alpha_{T}\left(\cos \psi_{T} \sin \phi_{T}-\cos \phi_{T} \sin \psi_{T} \sin \theta_{T}\right)-V_{T} \sin \beta_{T}\left(\cos \phi_{T} \cos \psi_{T}\right.$ $\left.+\sin \phi_{T} \sin \psi_{T} \sin \theta_{T}\right)-\left.V_{T} \cos \alpha_{T} \cos \beta_{T} \cos \theta_{T} \sin \psi_{T}\right|_{o}$

$\mathrm{Y}_{V_{T_{o}}}(t)=\sin \beta_{T}\left(\cos \phi_{T} \cos \psi_{T}+\sin \phi_{T} \sin \psi_{T} \sin \theta_{T}\right)-\cos \beta_{T} \sin \alpha_{T}\left(\cos \psi_{T} \sin \phi_{T}\right.$ $\left.-\cos \phi_{T} \sin \psi_{T} \sin \theta_{T}\right)+\left.\cos \alpha_{T} \cos \beta_{T} \cos \theta_{T} \sin \psi_{T}\right|_{o}$

$\mathrm{Y}_{\beta_{T_{o}}}(t)=V_{T} \cos \beta_{T}\left(\cos \phi_{T} \cos \psi_{T}+\sin \phi_{T} \sin \psi_{T} \sin \theta_{T}\right)+V_{T} \sin \alpha_{T} \sin \beta_{T}\left(\cos \psi_{T} \sin \phi_{T}\right.$ $\left.-\cos \phi_{T} \sin \psi_{T} \sin \theta_{T}\right)-\left.V_{T} \cos \alpha_{T} \sin \beta_{T} \cos \theta_{T} \sin \psi_{T}\right|_{o}$

$\mathrm{Y}_{\alpha_{T_{o}}}(t)=-V_{T} \cos \alpha_{T} \cos \beta_{T}\left(\cos \psi_{T} \sin \phi_{T}-\cos \phi_{T} \sin \psi_{T} \sin \theta_{T}\right)-\left.V_{T} \cos \beta_{T} \sin \alpha_{T} \cos \theta_{T} \sin \psi_{T}\right|_{o}$

$\mathrm{Y}_{\theta_{T_{o}}}(t)=\left.V_{T} \sin \psi_{T}\left(\sin \beta_{T} \cos \theta_{T} \sin \phi_{T}-\cos \alpha_{T} \cos \beta_{T} \sin \theta_{T}+\cos \beta_{T} \cos \phi_{T} \sin \alpha_{T} \cos \theta_{T}\right)\right|_{o}$

$\mathrm{Y}_{\phi_{T_{o}}}(t)=-V_{T} \sin \beta_{T}\left(\cos \psi_{T} \sin \phi_{T}-\cos \phi_{T} \sin \psi_{T} \sin \theta_{T}\right)-V_{T} \cos \beta_{T} \sin \alpha_{T}\left(\cos \phi_{T} \cos \psi_{T}\right.$ $\left.+\sin \phi_{T} \sin \psi_{T} \sin \theta_{T}\right)\left.\right|_{o}$

$\mathrm{Y}_{\psi_{T_{o}}}(t)=V_{T} \cos \beta_{T} \sin \alpha_{T}\left(\sin \phi_{T} \sin \psi_{T}+\cos \phi_{T} \cos \psi_{T} \sin \theta_{T}\right)-V_{T} \sin \beta_{T}\left(\cos \phi_{T} \sin \psi_{T}\right.$ $\left.-\cos \psi_{T} \sin \phi_{T} \sin \theta_{T}\right)+\left.V_{T} \cos \alpha_{T} \cos \beta_{T} \cos \psi_{T} \cos \theta_{T}\right|_{o}$ 


\section{APPENDIX E \\ SIMULATION RESULTS REPEATED AT OBSERVATION RELATIVE REFUELING POSITION}


This appendix repeats the evaluation tests established in Chapter 5 at the observation relative position to the tanker, rather than the contact refueling relative position. While the contact position is located directly aft of the tanker $(-35.5 \mathrm{~m}$ aft in axial deviation along the tanker fuselage reference line, $0 \mathrm{~m}$ lateral displacement from the fuselage reference line along the tanker wing reference line, and $8.5 \mathrm{~m}$ elevation in vertical displacement from the fuselage reference line), the observation position is further aft with a significant lateral offset (-59.13 m aft, $56.33 \mathrm{~m}$ lateral displacement, $0 \mathrm{~m}$ elevation). This difference in relative position raises two considerations. One, the observation position effectively increases the moment arm when considering a fixed coordinate relative to the tanker body, and motion of the tanker amplifies the spatial change of this position as the arm is extended further from the center (tanker body frame origin). Consider the partial derivatives that contain the coordinates of the relative position presented in Appendix D, which describe the influence of tanker motion on the receiver in the $\mathbf{H}(t)$ matrix as a function of the moment arm. The axial deviation rate, or rate of change in the $x$ direction, is contingent on the tanker angular velocity components of pitch rate and yaw rate by the $z$ and $y$ moment arms, respectively, as given by

$$
\begin{aligned}
& \mathrm{X}_{q_{T_{o}}}=-\left.z\right|_{o} \\
& \mathrm{X}_{r_{T_{o}}}=\left.y\right|_{o}
\end{aligned}
$$

The lateral deviation rate of the receiver with respect to the commanded relative position is impacted by the tanker roll rate (with $z$ as the moment arm) and yaw rate (with $x$ as the moment arm) given by,

$$
\begin{gathered}
\mathrm{Y}_{p_{T_{o}}}=\left.z\right|_{o} \\
\mathrm{Y}_{r_{T_{o}}}=-\left.x\right|_{o}
\end{gathered}
$$


Finally, the receiver elevation deviation rate is sensitive to the tanker roll rate (with $y$ as the moment arm) and pitch rate (with $x$ as the moment arm) given by,

$$
\begin{aligned}
& \mathrm{Z}_{p_{T_{o}}}=-\left.y\right|_{o} \\
& \mathrm{Z}_{q_{T_{o}}}=\left.x\right|_{o}
\end{aligned}
$$

Therefore, due to the fact that the observation position includes a zero elevation difference while the contact position is offset $8.5 \mathrm{~m}$ in $z$, one would expect a reduced sensitivity in the axial and lateral deviation for changes in the tanker body pitch rate and roll rate, respectively. However, the $x$ and $y$ coordinates are dramatically increased at the observation position, which according to the above partial derivatives, amplifies the impact on all axes of the station-keeping task due to tanker motion (i.e. changes in angular velocity). Refer to Fig. (E.1) which overlays station-keeping of contact position versus observation position when the tanker performs a 30 degree bank to the right, with the refined performance index and the absence of inertial wind. Note this comparison is conducted for the new Friedland control law alone, as these results exhibited the best tracking performance. The contact position time history is extracted from Fig. (5.24), previously presented in Chapter 5. Note the phase plane plot is modified to represent errors from the commanded position in order to allow correlation between the two responses at different relative positions. Performance is noticeably degraded and adequate tracking is not achieved in that the previously established box is exceeded in all axes when at the observation position. This result in degraded station-keeping performance at the observation relative position is considered due to the increased moment arms as aforementioned in the analysis of the partial derivative expansions. Recognizably, station-keeping at the observation position will involve a different set of tracking requirements when compared to the refueling contact position. The equivalent tracking criteria are used in this research solely to allow comparison of performance at the two relative positions. 

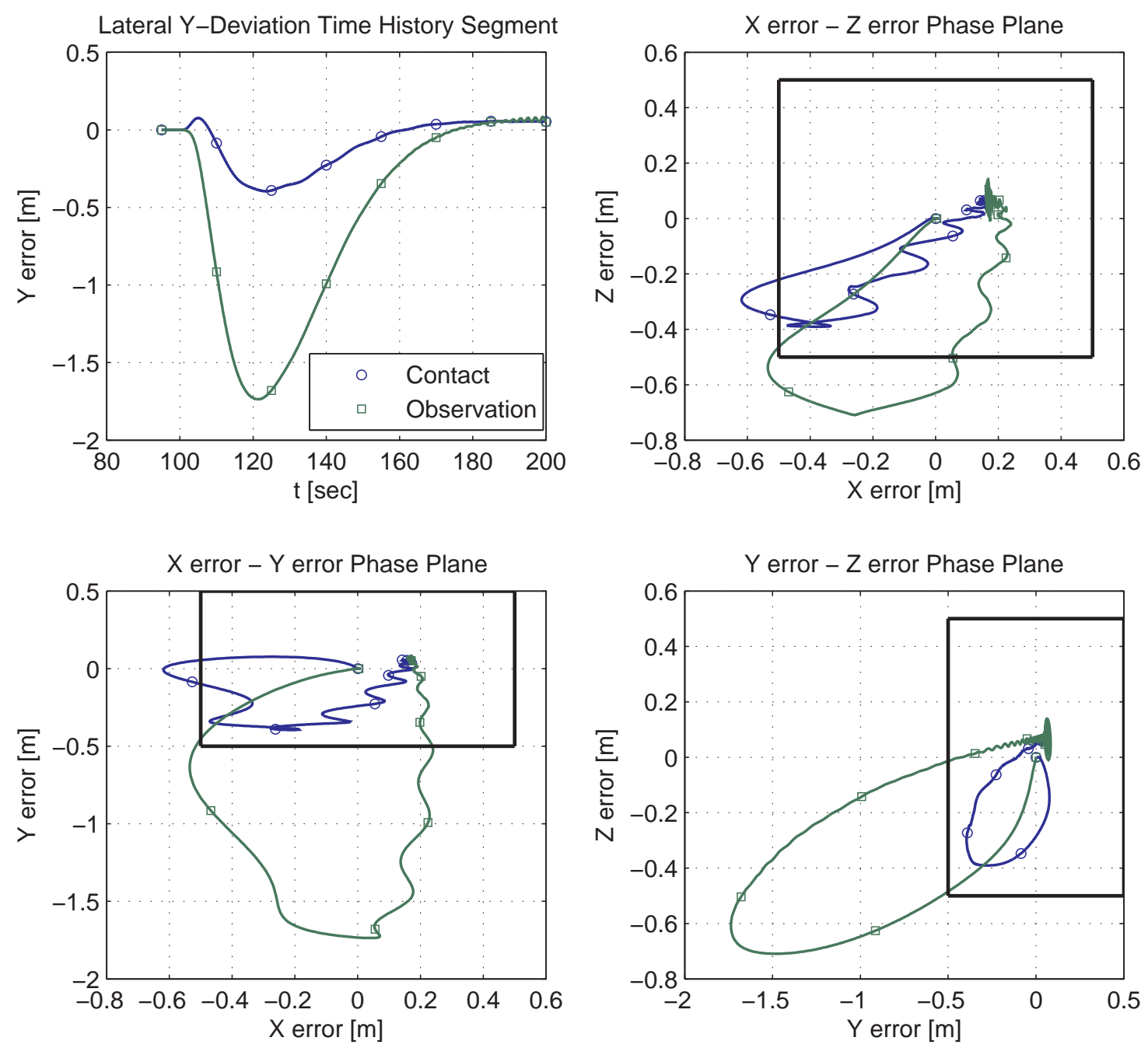

Figure E.1. Revised Weight Q, R: Contact and Observation Position Phase Plane Errors LQR MIMO + Friedland Control Only for Nonlinear Simulation (Interactive Flow Field Only) while Tanker Performs a 30 Degree Bank. 
The second consideration regarding the impact of maintaining observation position rather than contact position is the expectation of decreased aerodynamic coupling, due to the receiver flying with a lateral and axial offset position. Obviously, the receiver will be less susceptible to disturbance caused by the non-uniform vortex-induced tanker wind field when further away from the tanker. In other words, with reduced exposure to this turbulent flow field, receiver station-keeping at the observation position should improve and require less control effort. Fig. (E.2) compares the wind environment on the receiver between the two relative positions, for the time history data used in the phase plane presentation previously discussed. (Note for the purpose of legibility, the legend on Fig. (E.2) has been omitted. Refer to Fig. (E.1) in that the blue lines with circle markers correspond to contact position while the green lines with square markers correspond to the results for the receiver at the observation position). Clearly, the magnitude of wind disturbance is significantly less at the observation position as anticipated. Therefore, with other disturbances aside (prevailing winds and tanker acceleration), one would expect station-keeping performance to be superior at the observation position for tracking a tanker while in straight and level flight. 

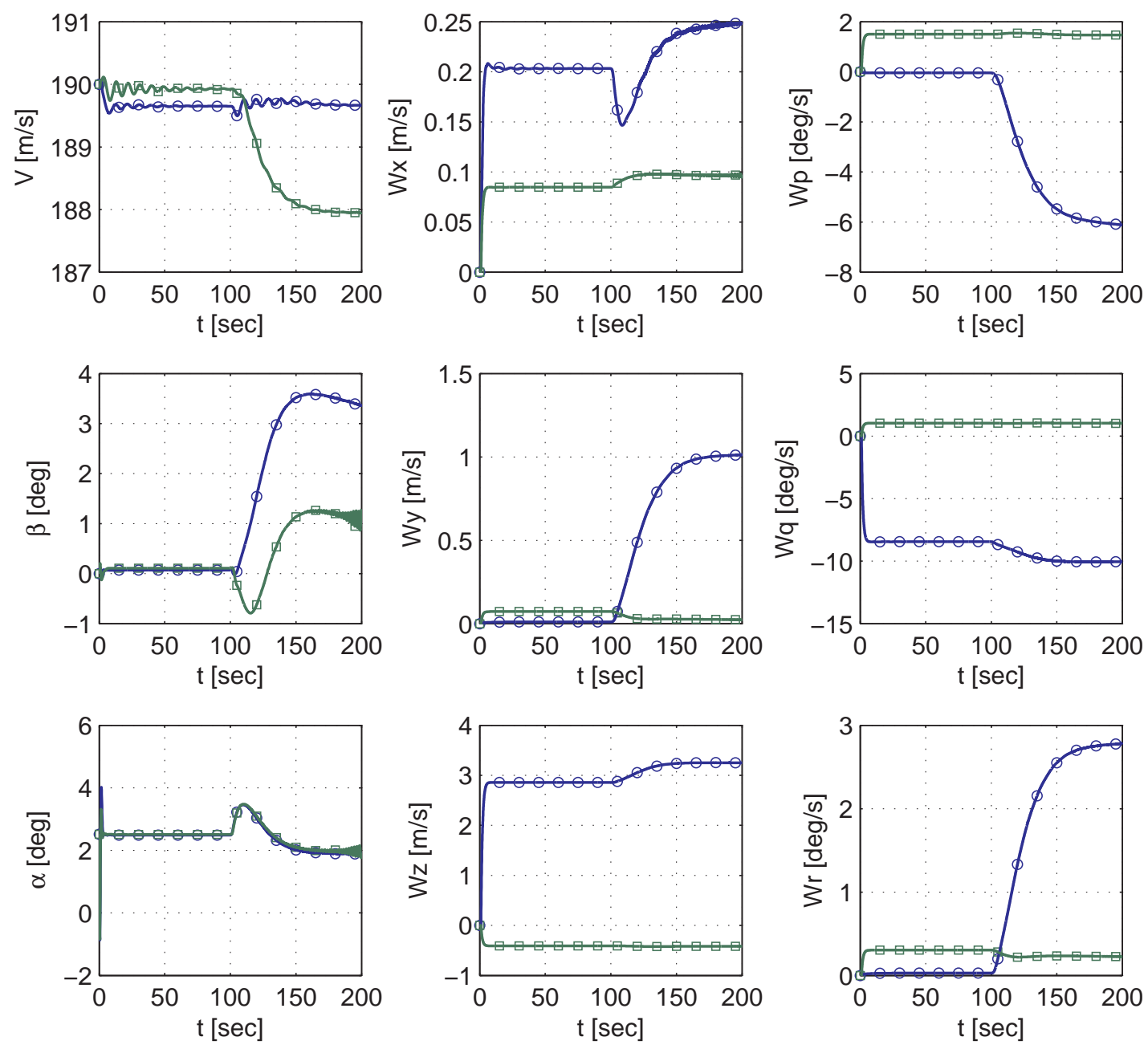

Figure E.2. Revised Weight Q, R: $V, \beta, \alpha$, and Winds Comparison (Contact Versus Observation Position) for Nonlinear Simulation (Interactive Flow Field Only) LQR MIMO + Friedland Control Disturbance Rejection while Tanker Performs a 30 Degree Bank. 
The following figures in this appendix are presented as raw data in that all evaluation techniques have been previously discussed in Chapter 5. Again, the linear and nonlinear results included are regenerated for the receiver station-keeping task while at the observation relative position to the tanker. Table E.1 provides a reference summary of results for the nonlinear simulation.

Table E.1. Summary of Results for Nonlinear Simulation with Receiver at Observation Position

\begin{tabular}{|c|c|c|c|c|c|c|c|}
\hline Winds & Maneuver & Control & Gain Set & Stable? & Max $\left|e_{x}\right|$ & Max $\left|e_{y}\right|$ & Max $\left|e_{z}\right|$ \\
\hline \hline Vortex Induced & $30 \mathrm{deg}$ & Base & Unity & No & - & - & - \\
\hline Vortex Induced & $30 \mathrm{deg}$ & Friedland & Unity & No & - & - & - \\
\hline Vortex Induced & $30 \mathrm{deg}$ & Base & Base & Yes & $5 \mathrm{~m}$ & $20 \mathrm{~m}$ & $2.5 \mathrm{~m}$ \\
\hline Vortex Induced & $30 \mathrm{deg}$ & Friedland & Base & Yes & $4 \mathrm{~m}$ & $6 \mathrm{~m}$ & $6 \mathrm{~m}$ \\
\hline Vortex Induced & $30 \mathrm{deg}$ & Base & Revised & No & - & - & - \\
\hline Vortex Induced & $30 \mathrm{deg}$ & Friedland & Revised & Yes & $0.5 \mathrm{~m}$ & $2 \mathrm{~m}$ & $1 \mathrm{~m}$ \\
\hline All & $15 \mathrm{deg}$ & Base & Base & Yes & $4 \mathrm{~m}$ & $8 \mathrm{~m}$ & $5 \mathrm{~m}$ \\
\hline All & $15 \mathrm{deg}$ & Friedland & Base & Yes & $5 \mathrm{~m}$ & $6 \mathrm{~m}$ & $5 \mathrm{~m}$ \\
\hline All & $-15 \mathrm{deg}$ & Base & Base & Yes & $4 \mathrm{~m}$ & $12 \mathrm{~m}$ & $6 \mathrm{~m}$ \\
\hline All & $-15 \mathrm{deg}$ & Friedland & Base & Yes & $3 \mathrm{~m}$ & $4 \mathrm{~m}$ & $5 \mathrm{~m}$ \\
\hline
\end{tabular}

E.1 Linear Model Results for Observation Relative Position Station-Keeping 

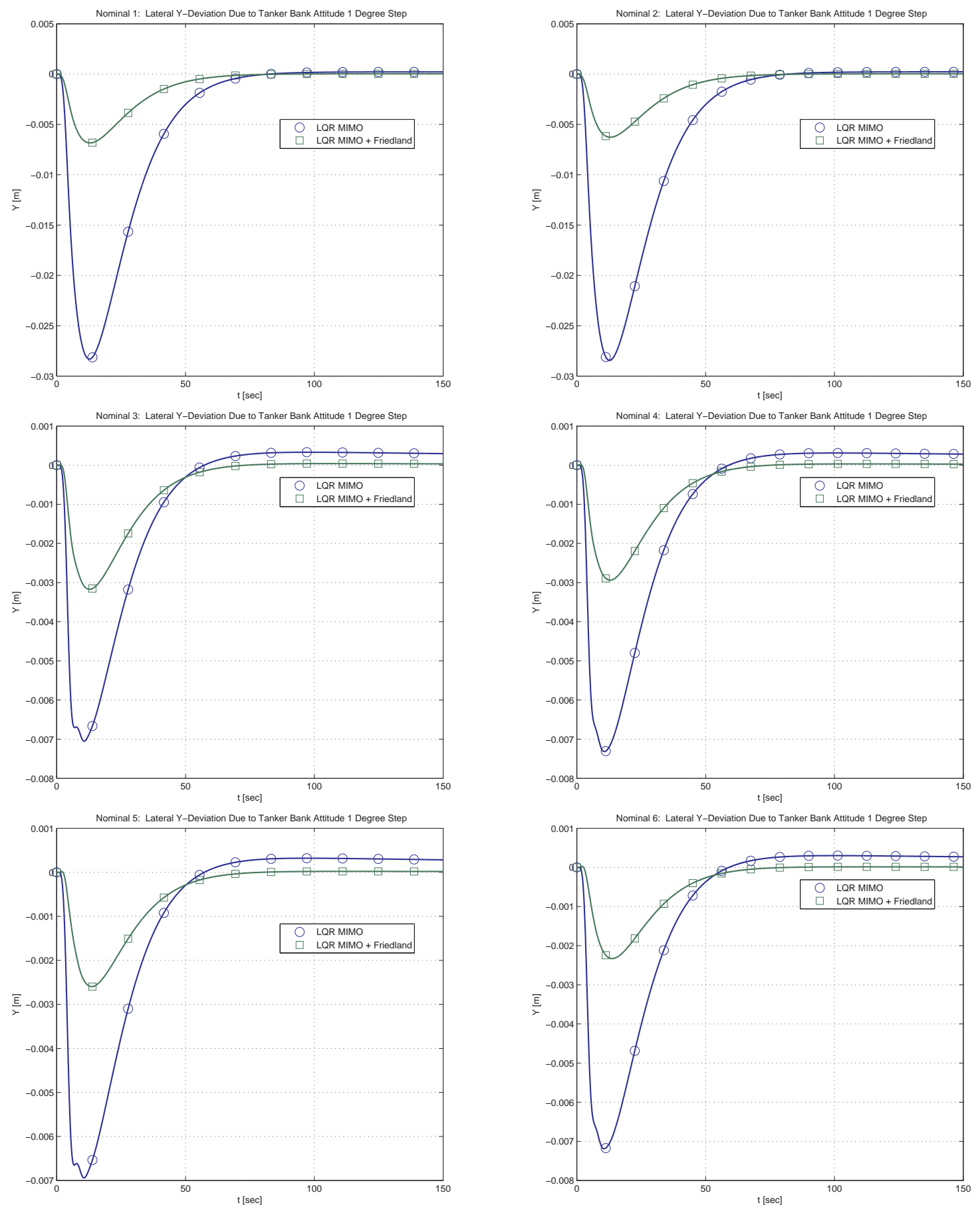

Figure E.3. Receiver Lateral (y) Deviation Response Due to Tanker Bank Attitude Command Unit Step [m/deg] per Nominal Condition (Unity Weight Q, R). 

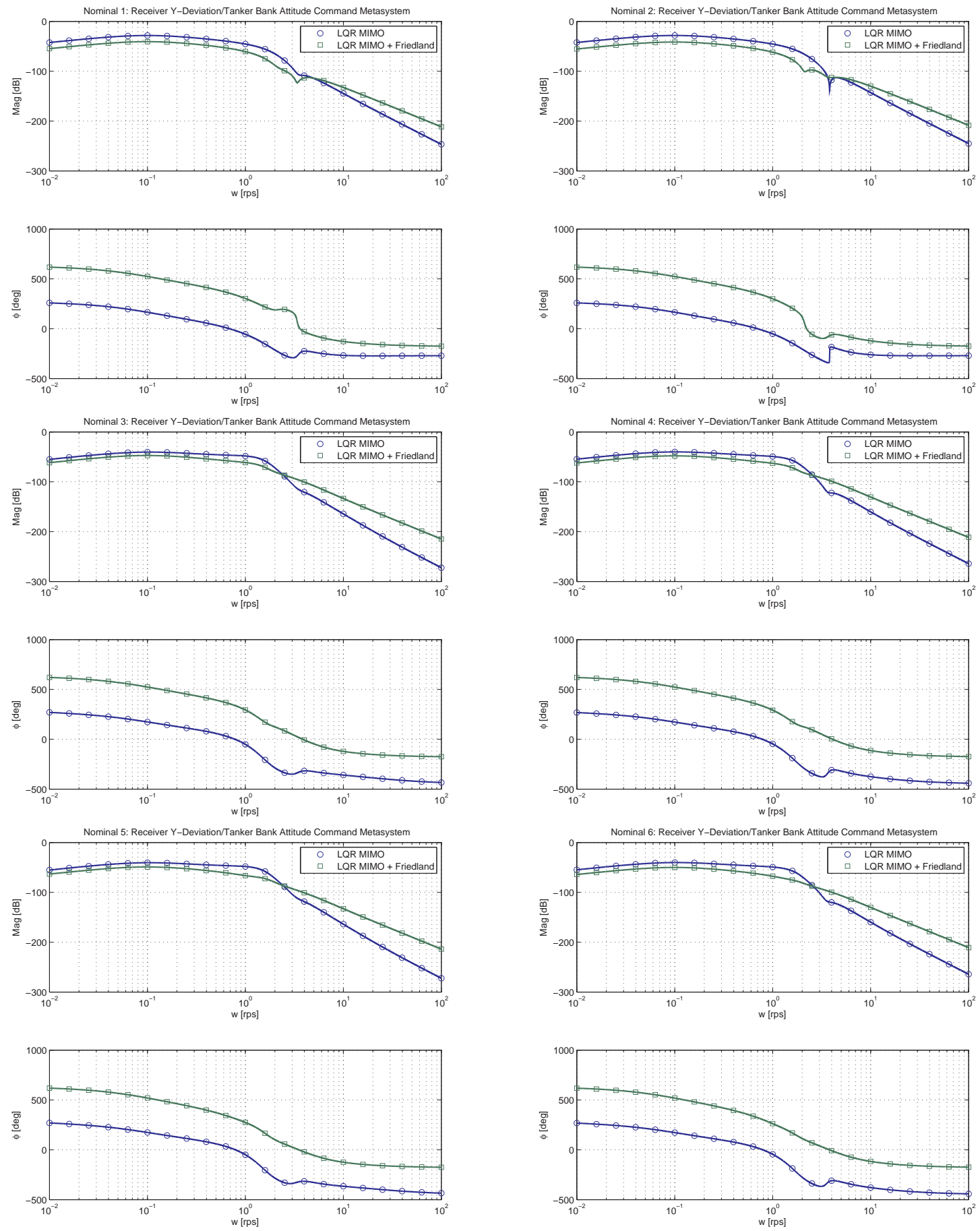

Figure E.4. Receiver Lateral (y) Deviation to Tanker Bank Attitude Command Metasystem Frequency Response per Nominal Condition (Unity Weight Q, R). 

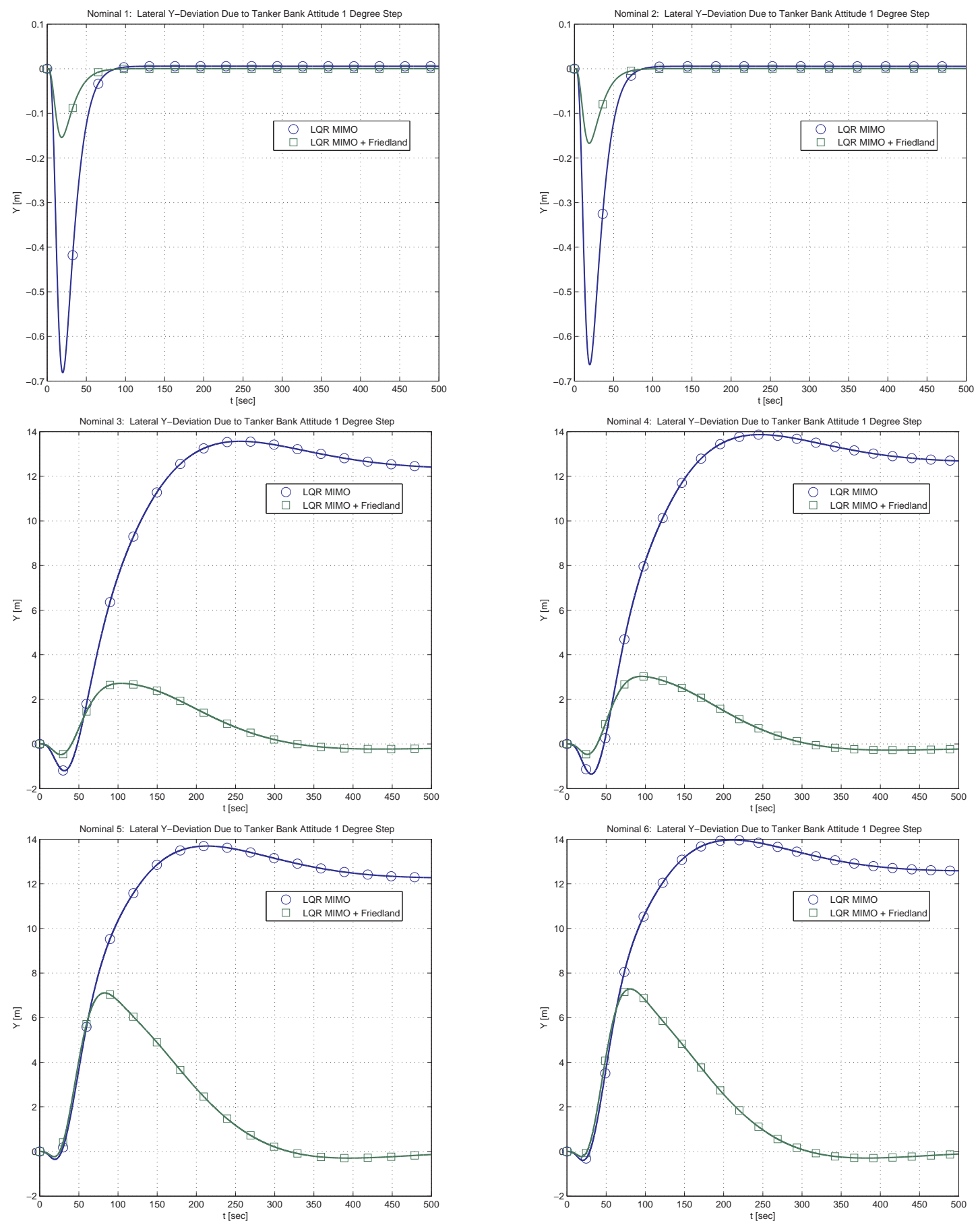

Figure E.5. Receiver Lateral (y) Deviation Response Due to Tanker Bank Attitude Command Unit Step [m/deg] per Nominal Condition (Currently Employed Q, R). 

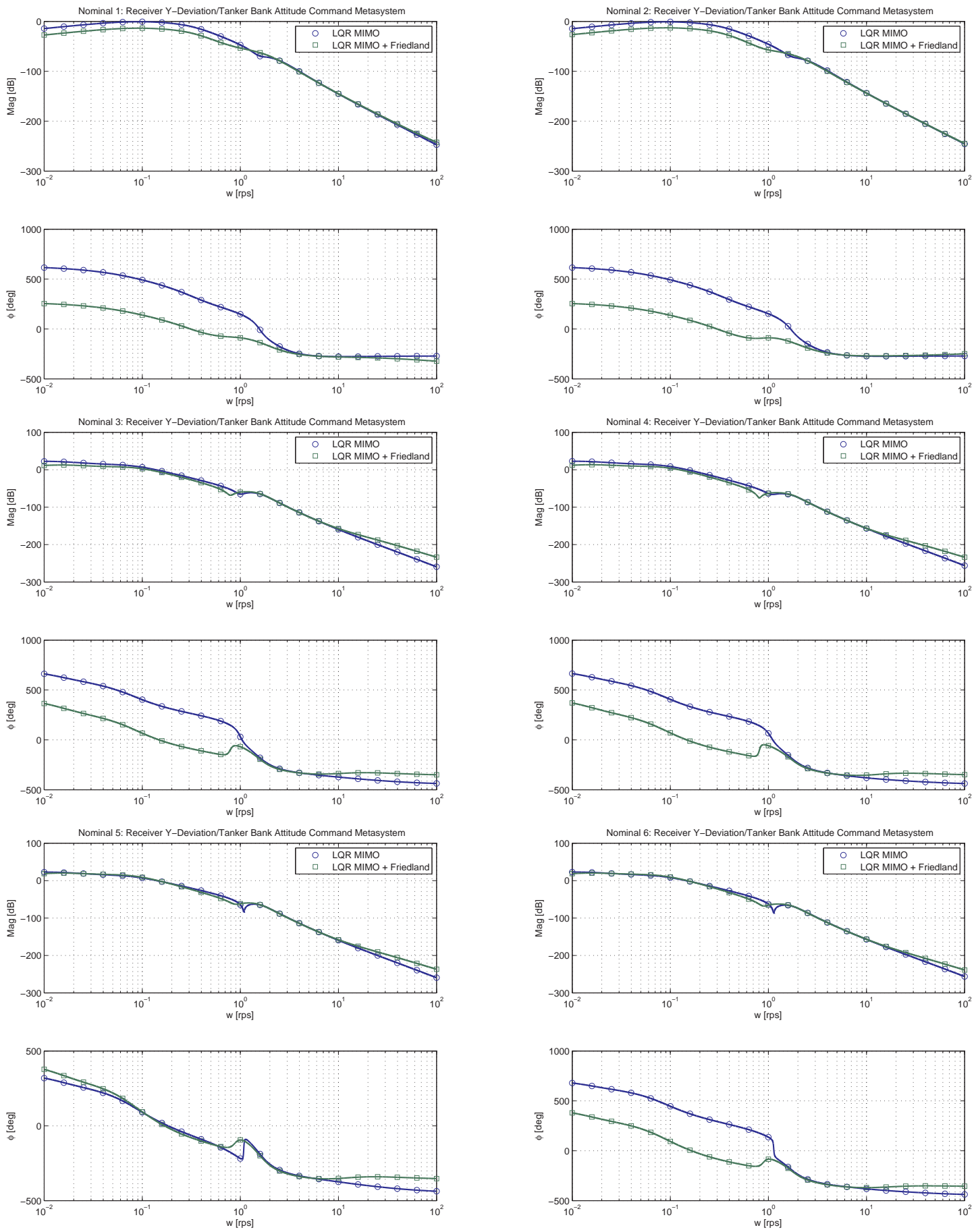

Figure E.6. Receiver Lateral (y) Deviation to Tanker Bank Attitude Command Metasystem Frequency Response per Nominal Condition (Currently Employed Q, R). 

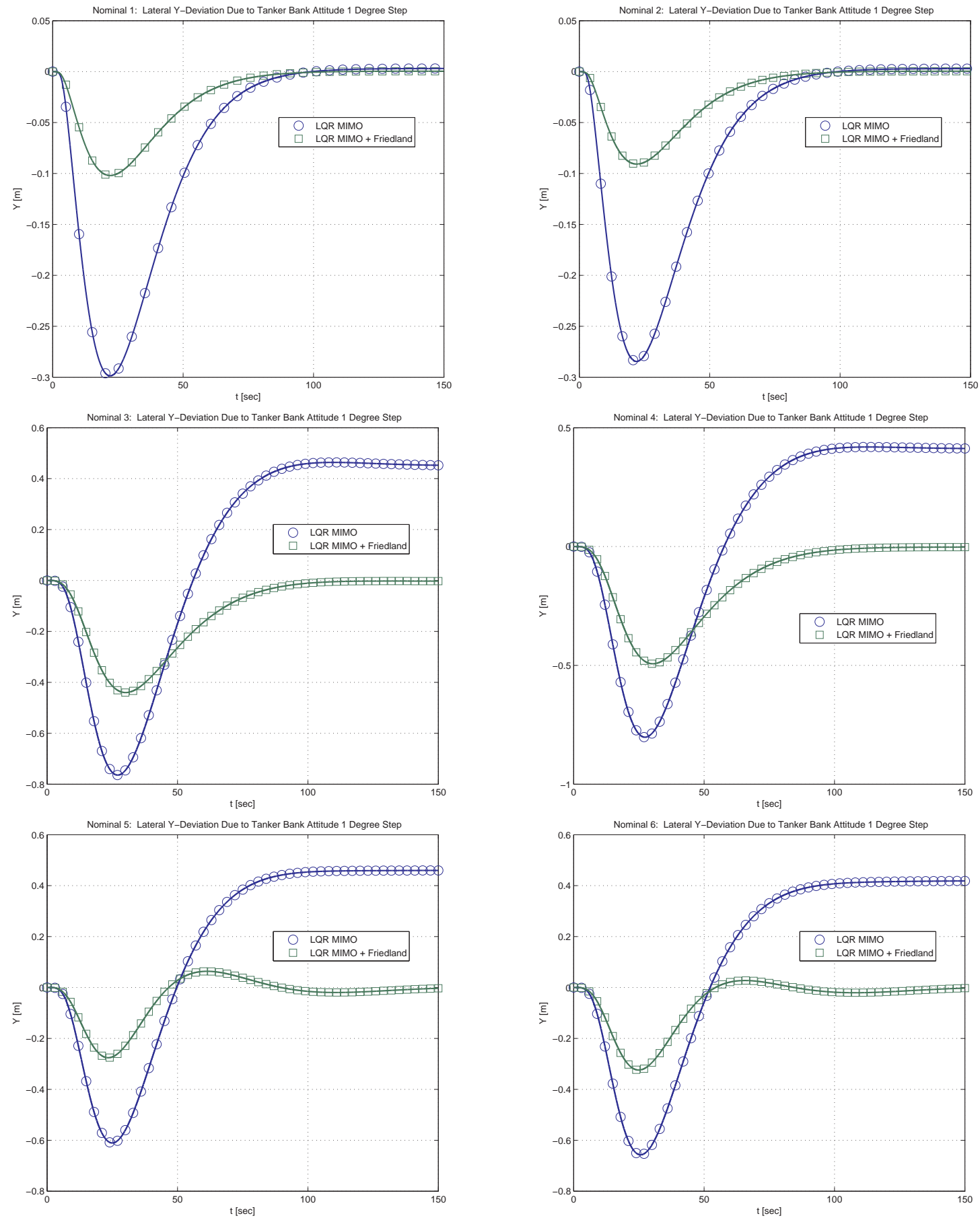

Figure E.7. Receiver Lateral (y) Deviation Response Due to Tanker Bank Attitude Command Unit Step [m/deg] per Nominal Condition (Revised Weight $\mathbf{Q}, \mathbf{R}$ ). 

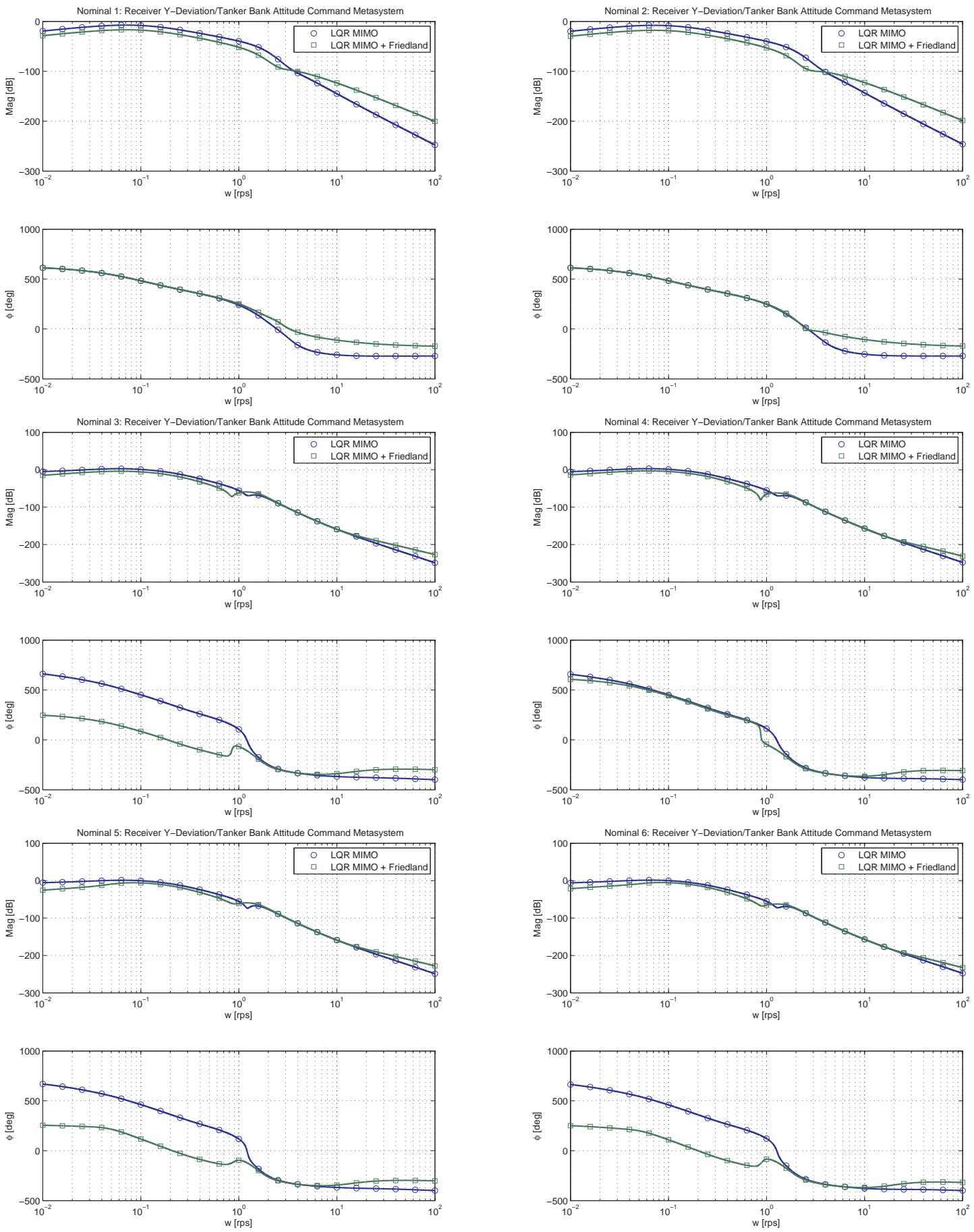

Figure E.8. Receiver Lateral (y) Deviation to Tanker Bank Attitude Command Metasystem Frequency Response per Nominal Condition (Revised Weight Q, R). 
E.2 Nonlinear Model Results

E.2.1 Results for Observation Relative Position Station-Keeping in the Presence of Tanker Flow Field

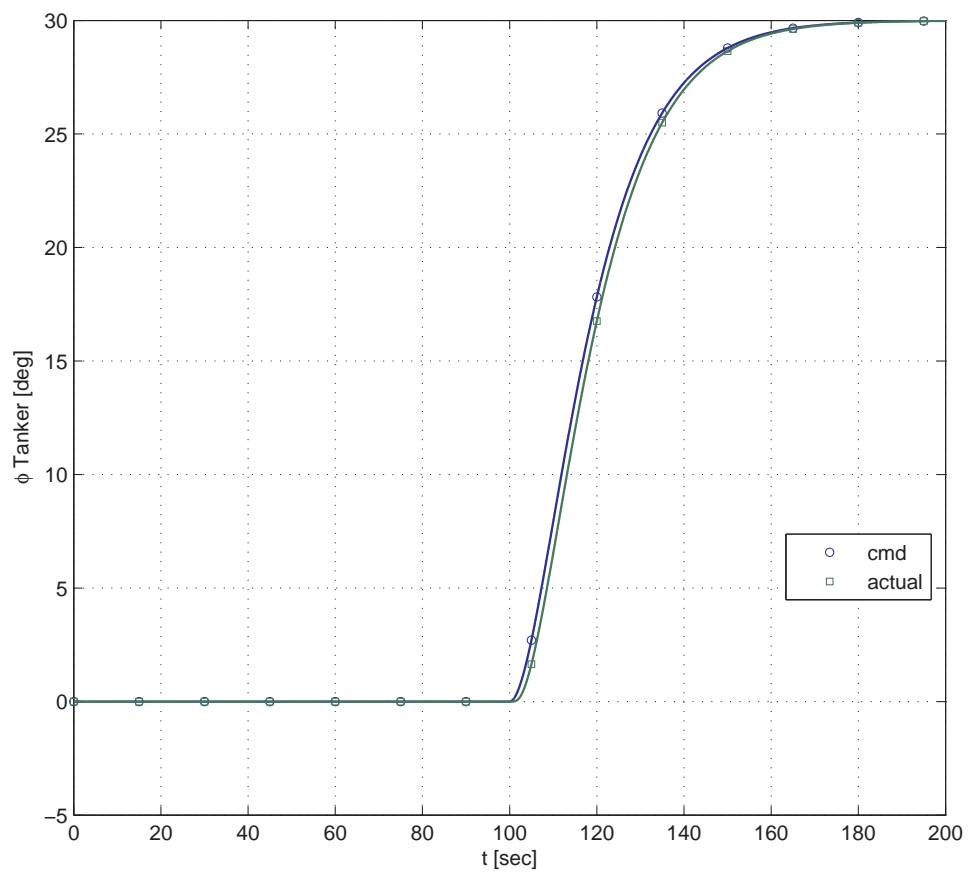

Figure E.9. Nonlinear Simulation Tanker Performing a 30 Degree Bank. 

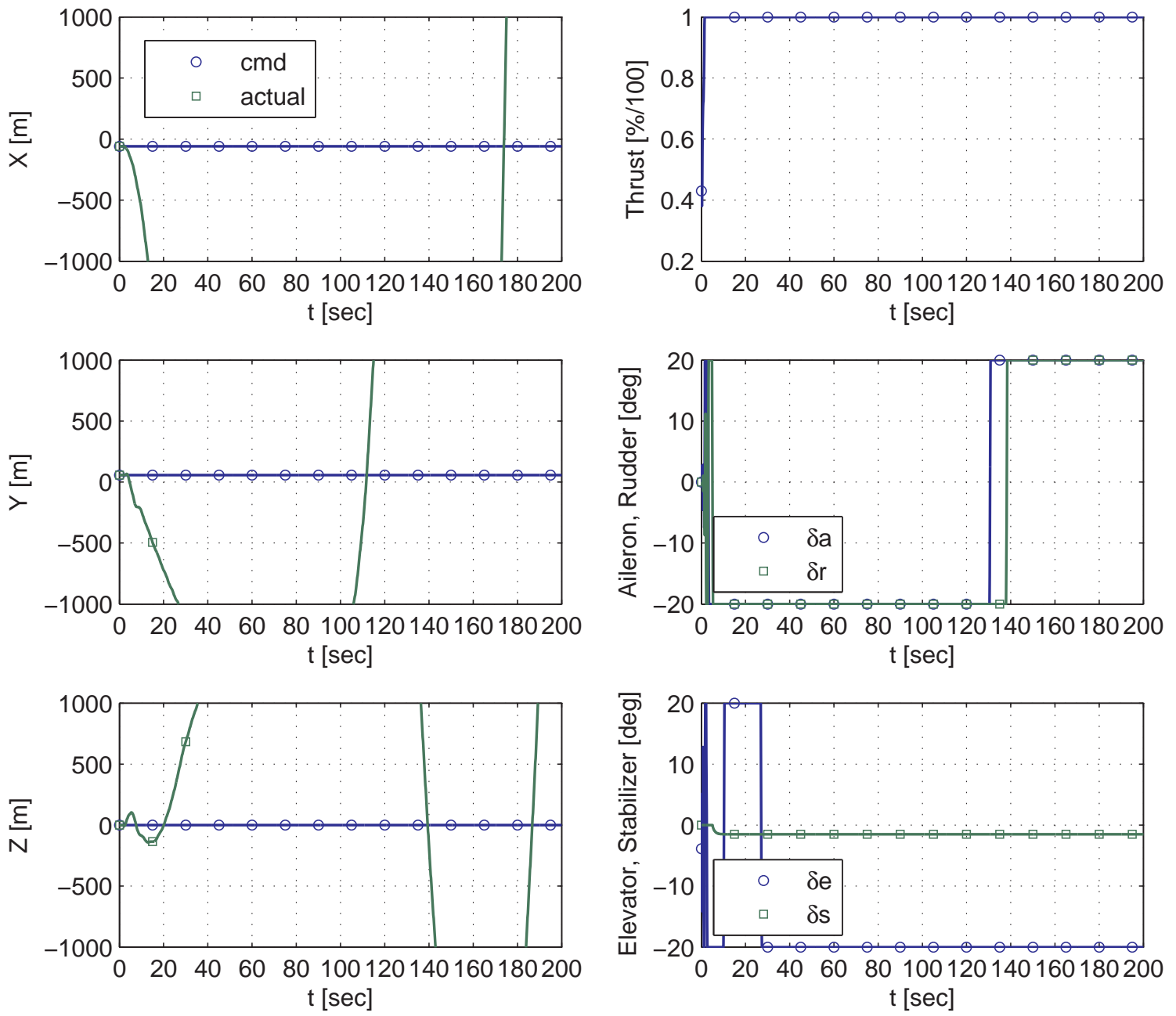

Figure E.10. Unity Weight Q, R: Station-Keeping and Effectors for Nonlinear Simulation (Interactive Flow Field Only) LQR MIMO Control while Tanker Performs a 30 Degree Bank. 

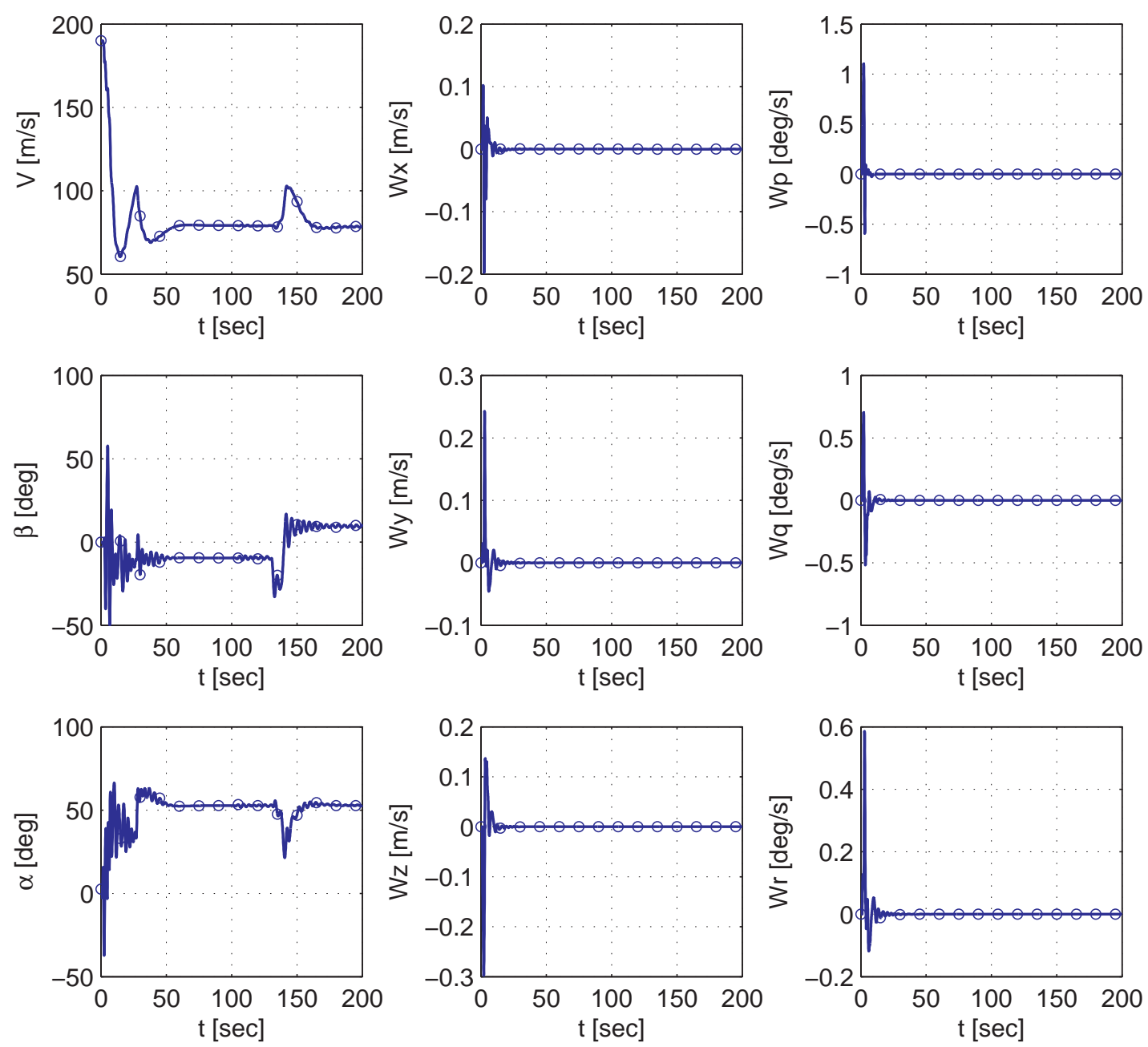

Figure E.11. Unity Weight Q, R: $V, \beta, \alpha$, and Winds for Nonlinear Simulation (Interactive Flow Field Only) LQR MIMO Control while Tanker Performs a 30 Degree Bank. 

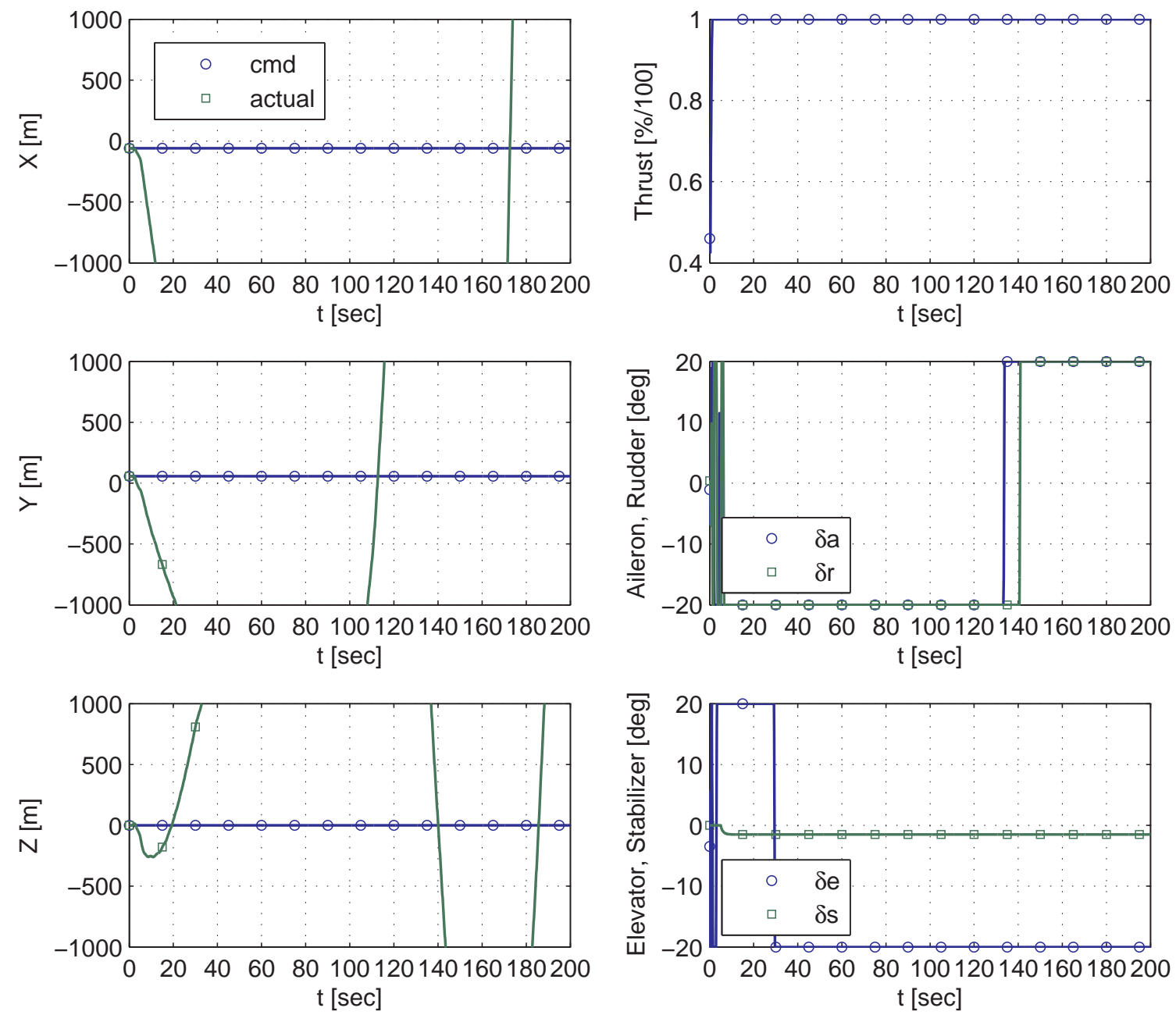

Figure E.12. Unity Weight Q, R: Station-Keeping and Effectors for Nonlinear Simulation (Interactive Flow Field Only) LQR MIMO + Friedland Control Disturbance Rejection while Tanker Performs a 30 Degree Bank. 

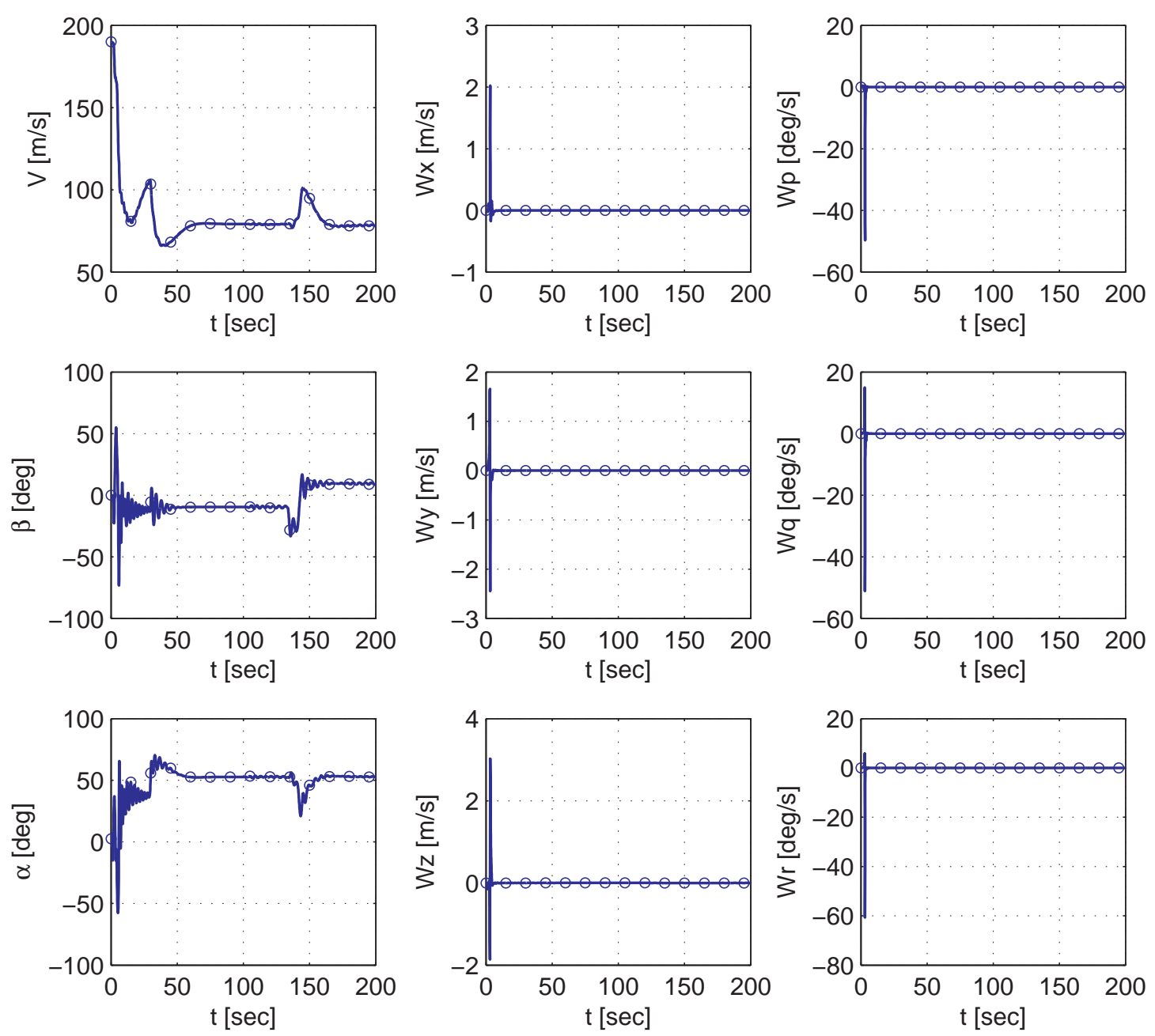

Figure E.13. Unity Weight Q, R: $V, \beta, \alpha$, and Winds for Nonlinear Simulation (Interactive Flow Field Only) LQR MIMO + Friedland Control Disturbance Rejection while Tanker Performs a 30 Degree Bank. 

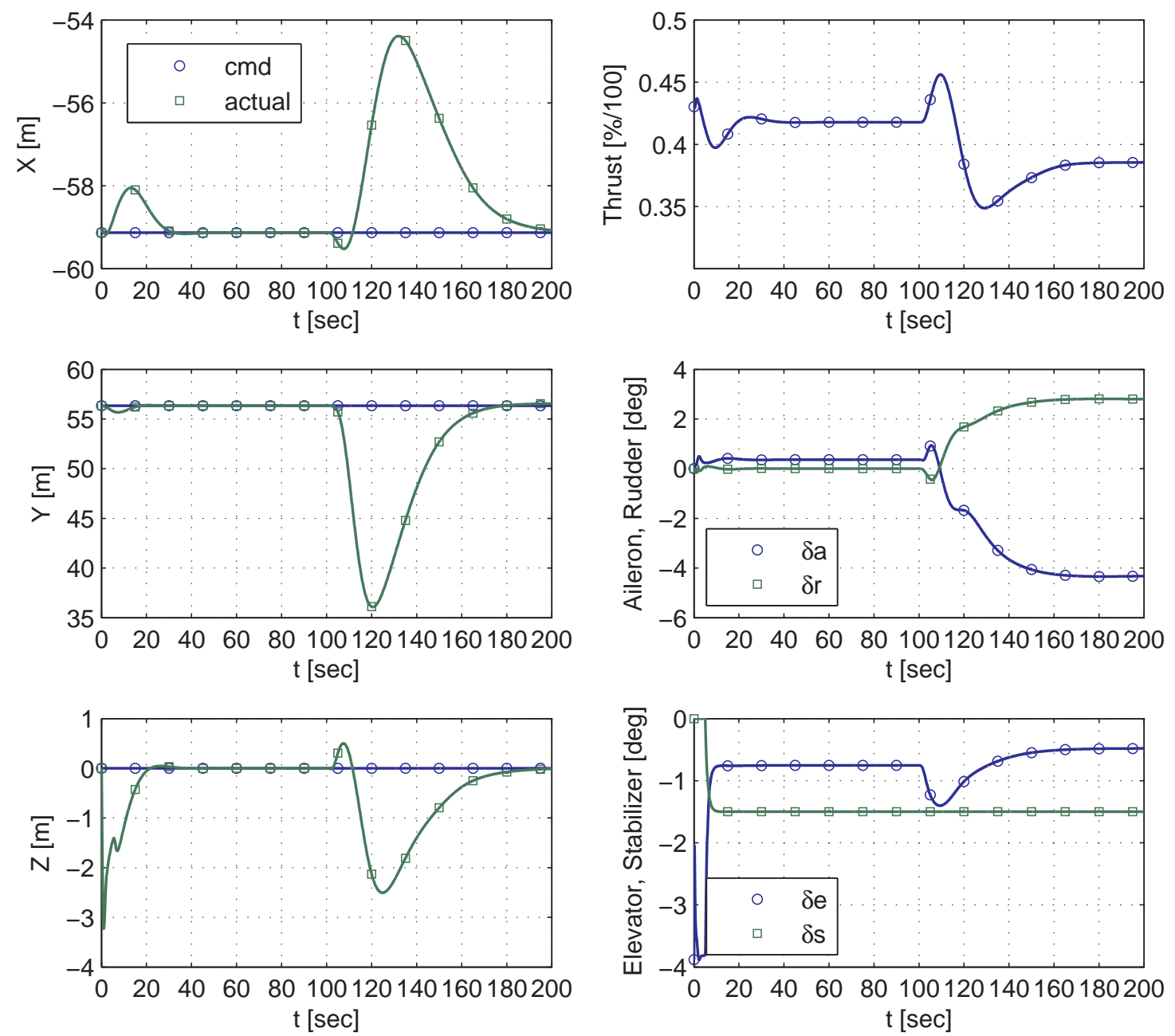

Figure E.14. Currently Employed Q, R: Station-Keeping and Effectors for Nonlinear Simulation (Interactive Flow Field Only) LQR MIMO Control while Tanker Performs a 30 Degree Bank. 

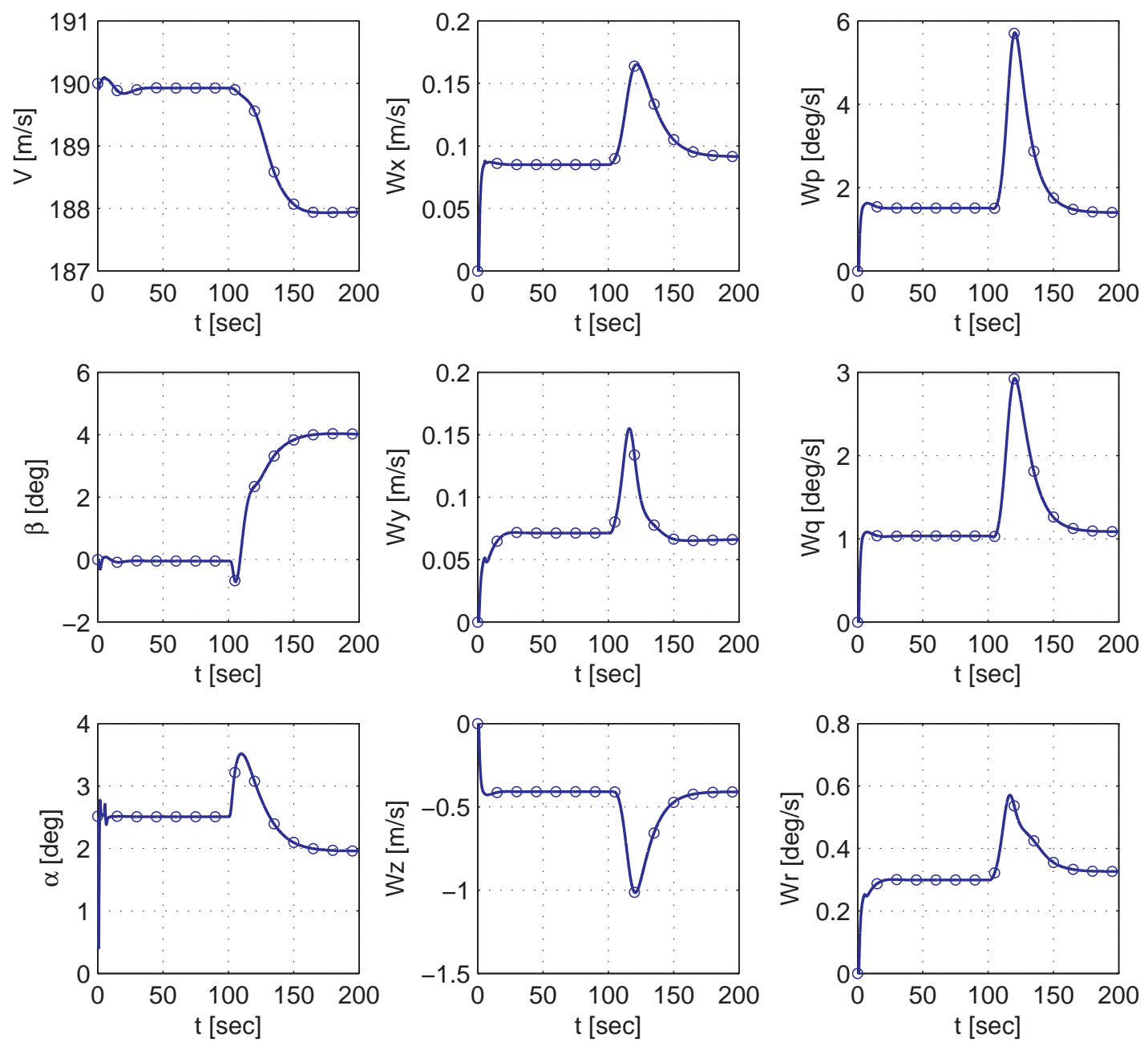

Figure E.15. Currently Employed Q, R: $V, \beta, \alpha$, and Winds for Nonlinear Simulation (Interactive Flow Field Only) LQR MIMO Control while Tanker Performs a 30 Degree Bank. 

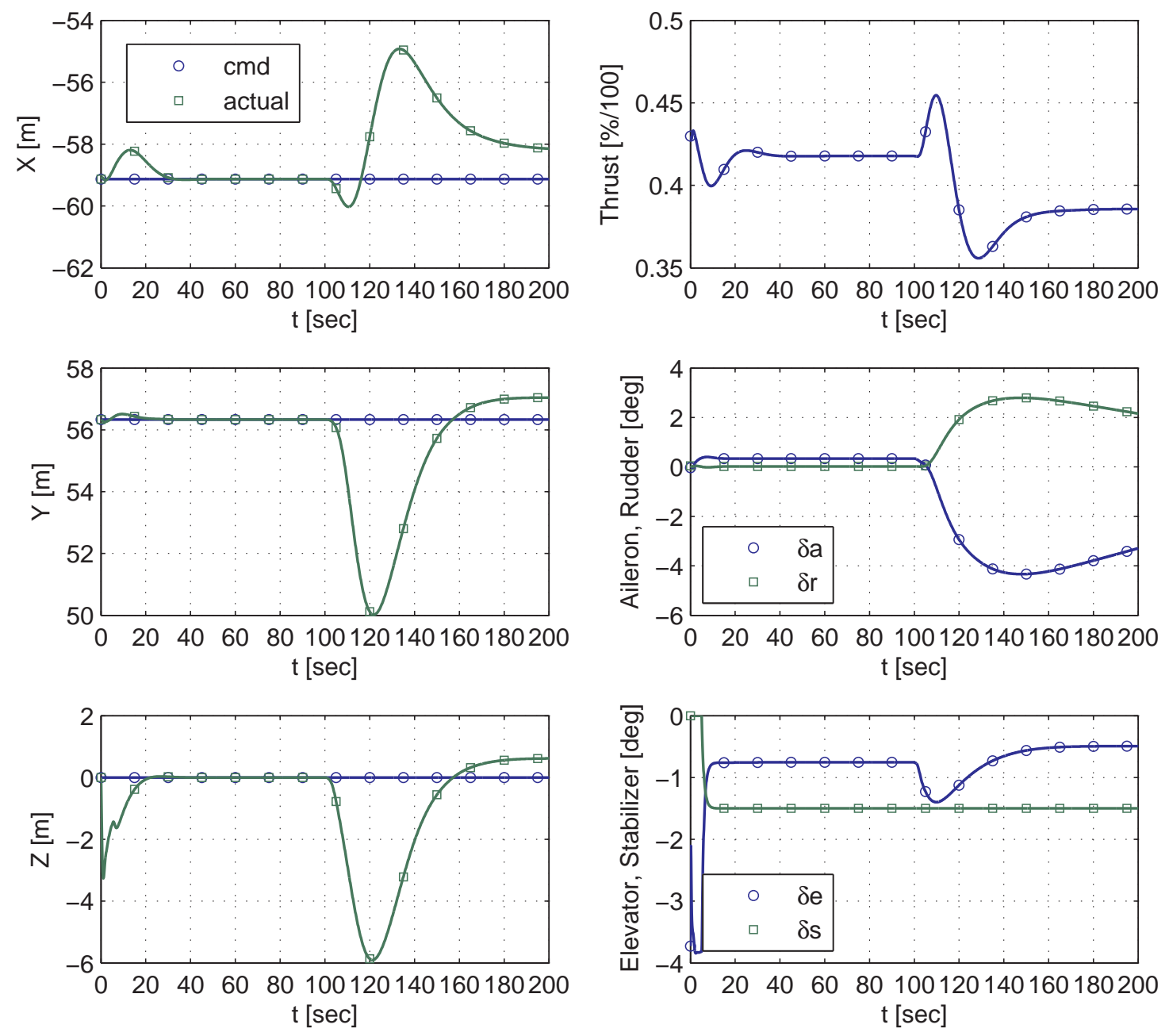

Figure E.16. Currently Employed Q, R: Station-Keeping and Effectors for Nonlinear Simulation (Interactive Flow Field Only) LQR MIMO + Friedland Control Disturbance Rejection while Tanker Performs a 30 Degree Bank. 

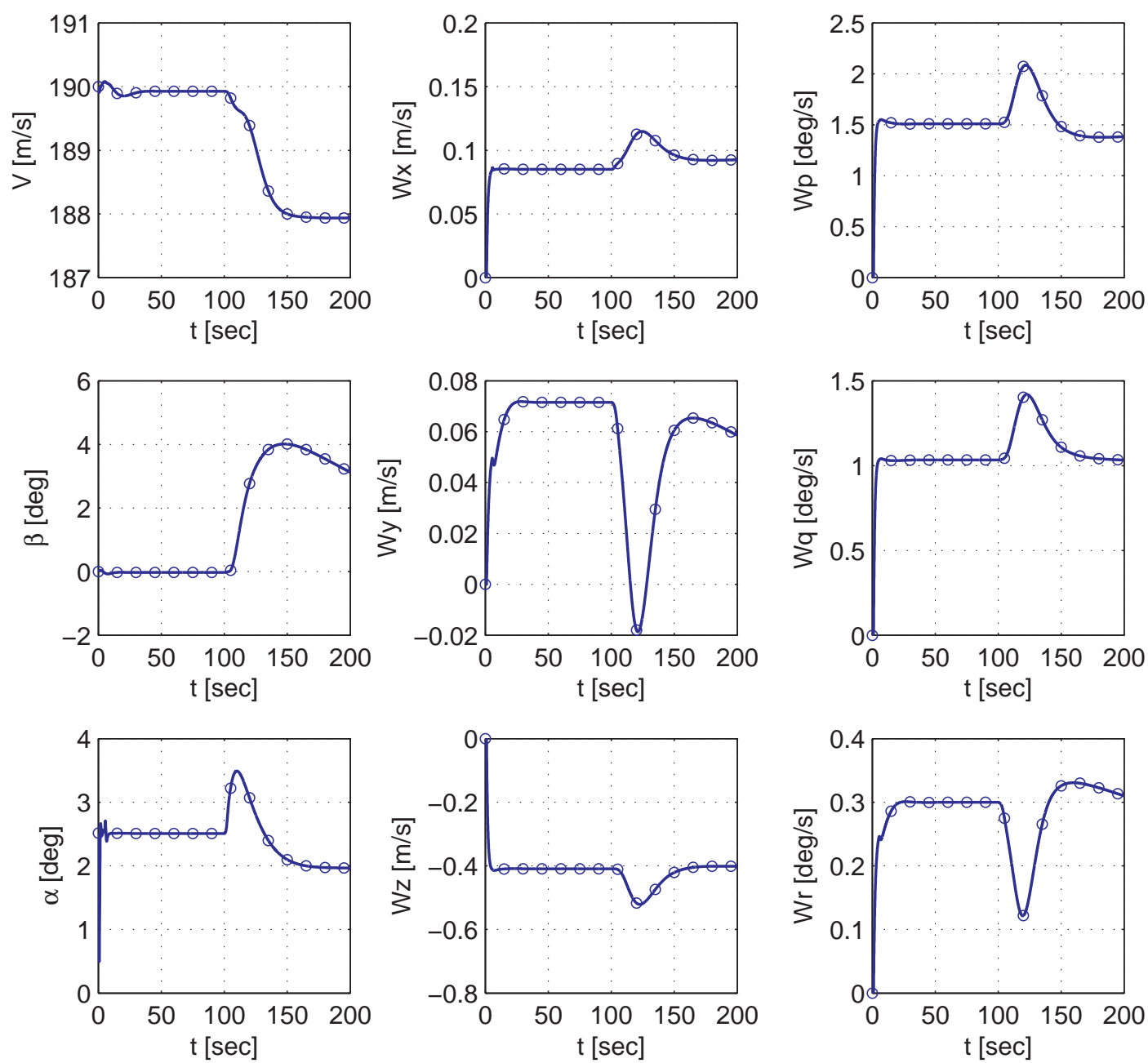

Figure E.17. Currently Employed Q, R: $V, \beta, \alpha$, and Winds for Nonlinear Simulation (Interactive Flow Field Only) LQR MIMO + Friedland Control Disturbance Rejection while Tanker Performs a 30 Degree Bank. 

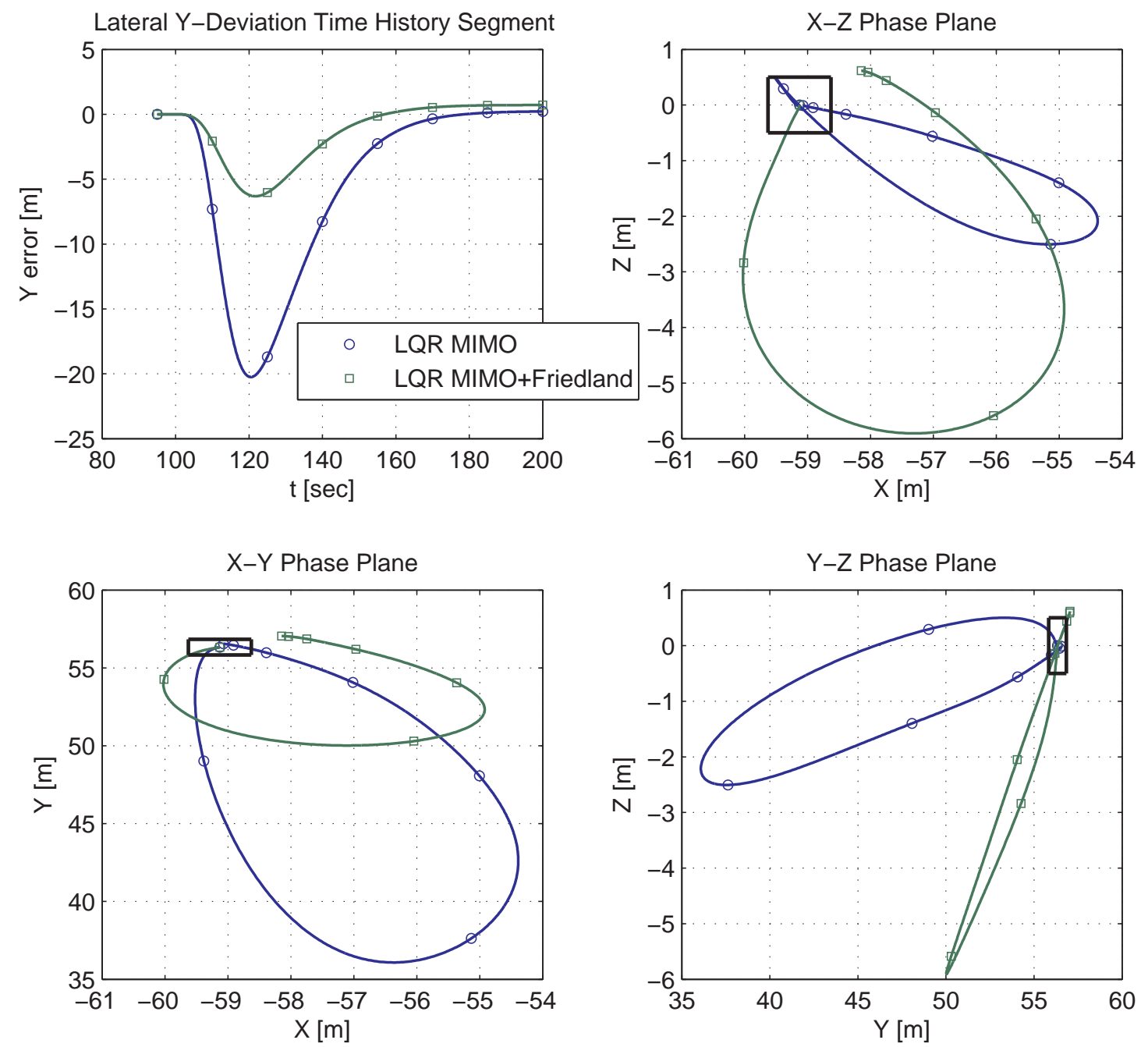

Figure E.18. Currently Employed Q, R: Phase Plane Controller Comparison for Nonlinear Simulation (Interactive Flow Field Only) while Tanker Performs a 30 Degree Bank. 

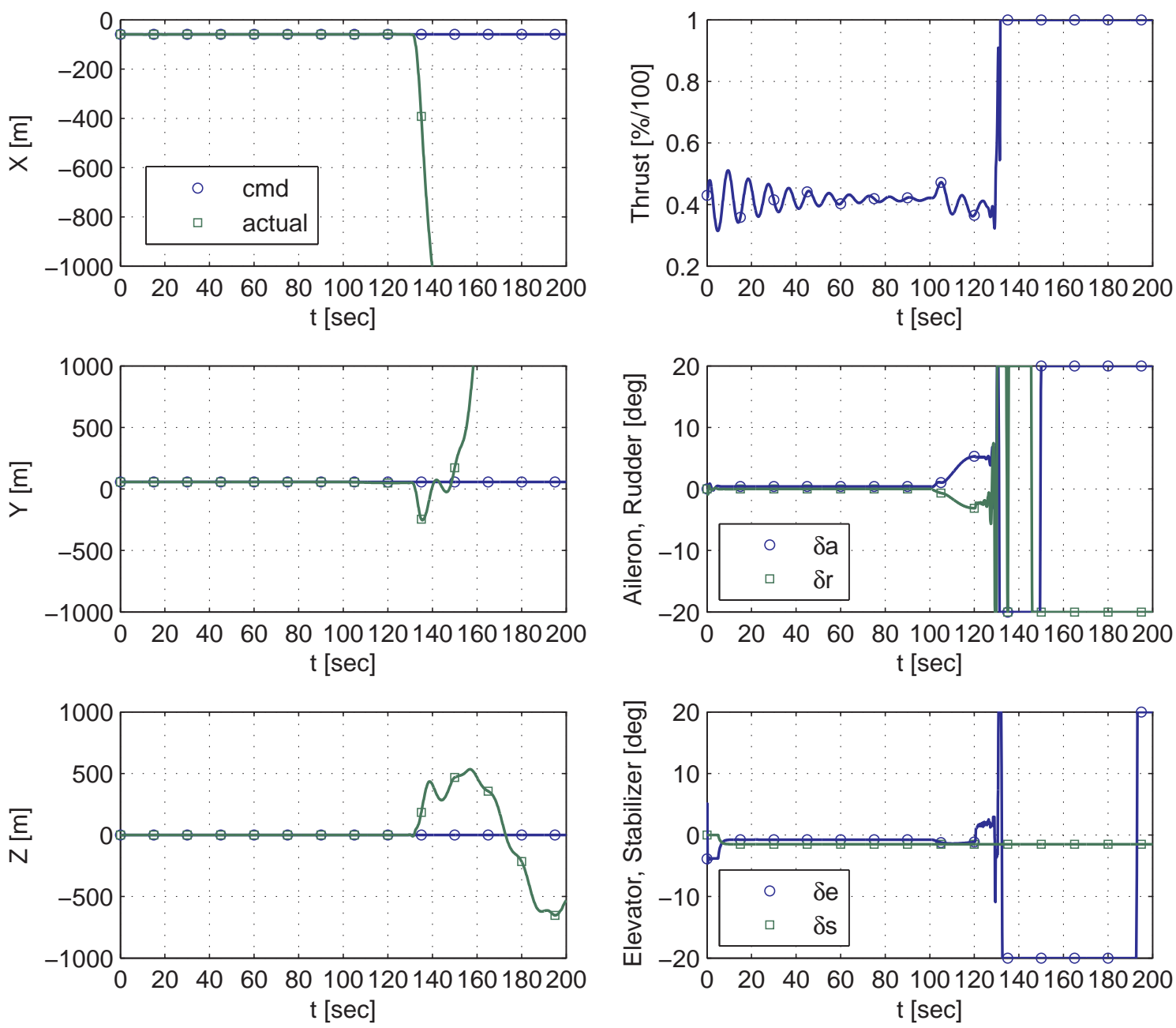

Figure E.19. Revised Weight Q, R: Station-Keeping and Effectors for Nonlinear Simulation (Interactive Flow Field Only) LQR MIMO Control while Tanker Performs a 30 Degree Bank. 

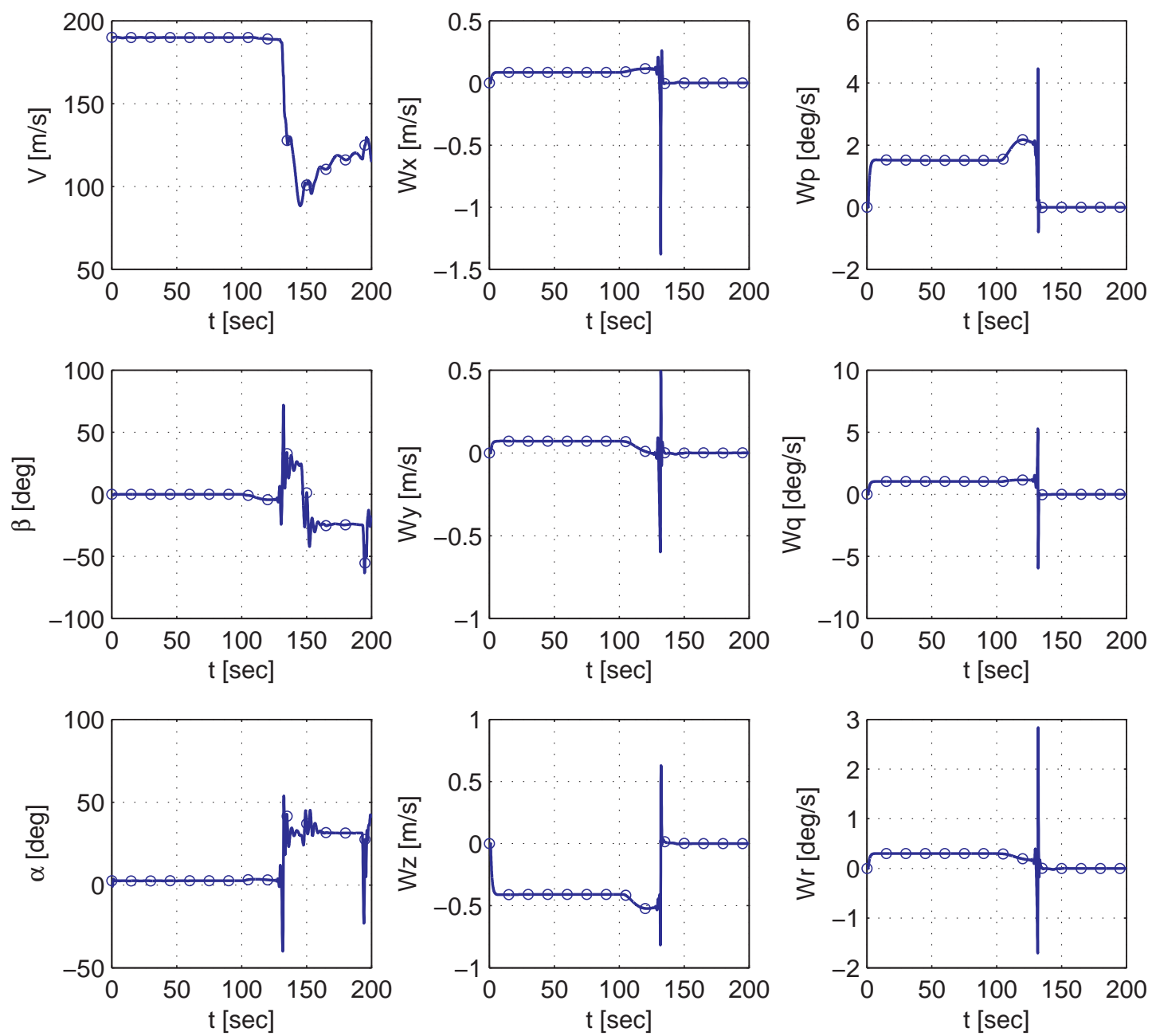

Figure E.20. Revised Weight Q, R: $V, \beta, \alpha$, and Winds for Nonlinear Simulation (Interactive Flow Field Only) LQR MIMO Control while Tanker Performs a 30 Degree Bank. 

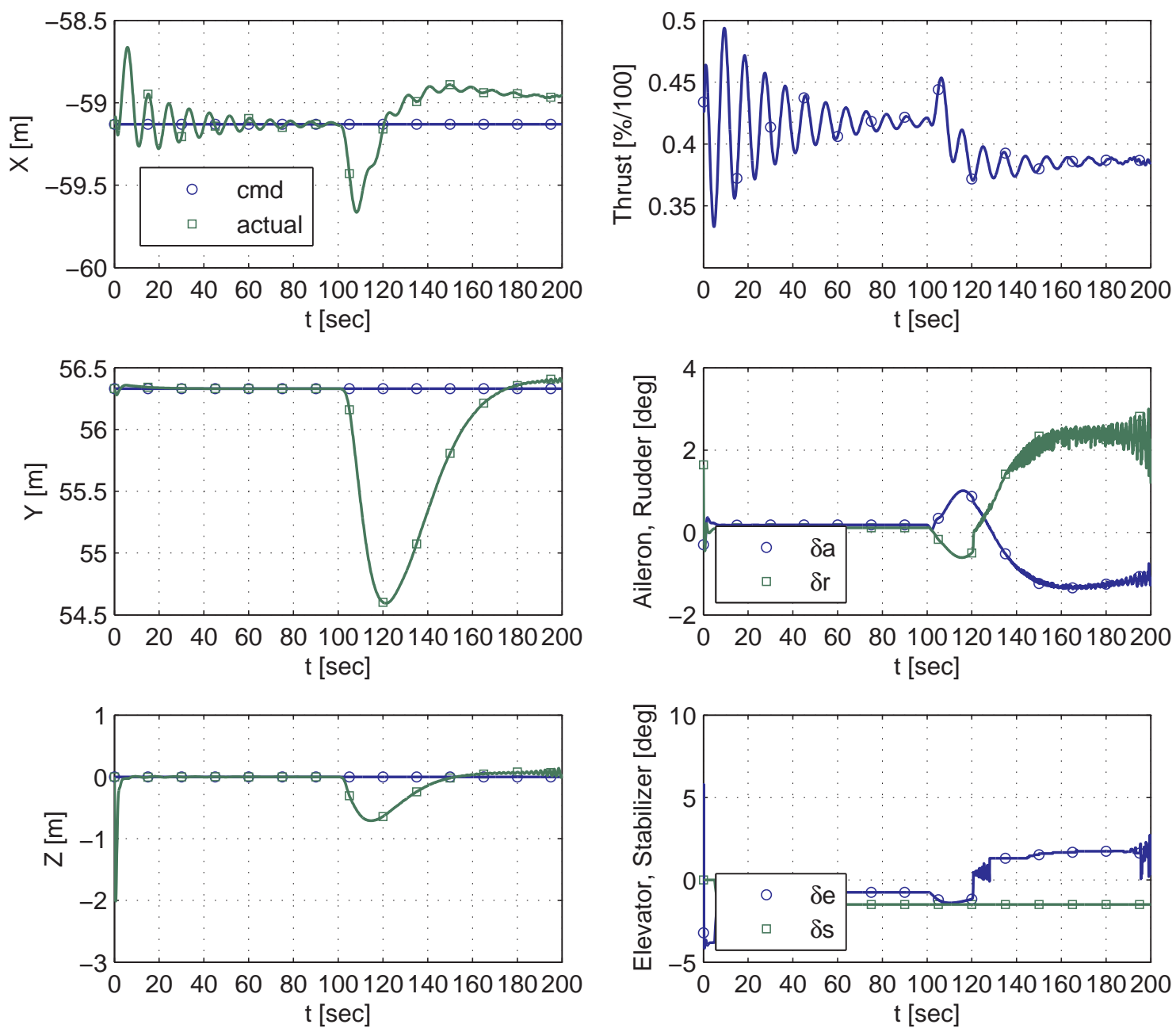

Figure E.21. Revised Weight Q, R: Station-Keeping and Effectors for Nonlinear Simulation (Interactive Flow Field Only) LQR MIMO + Friedland Control Disturbance Rejection while Tanker Performs a 30 Degree Bank. 

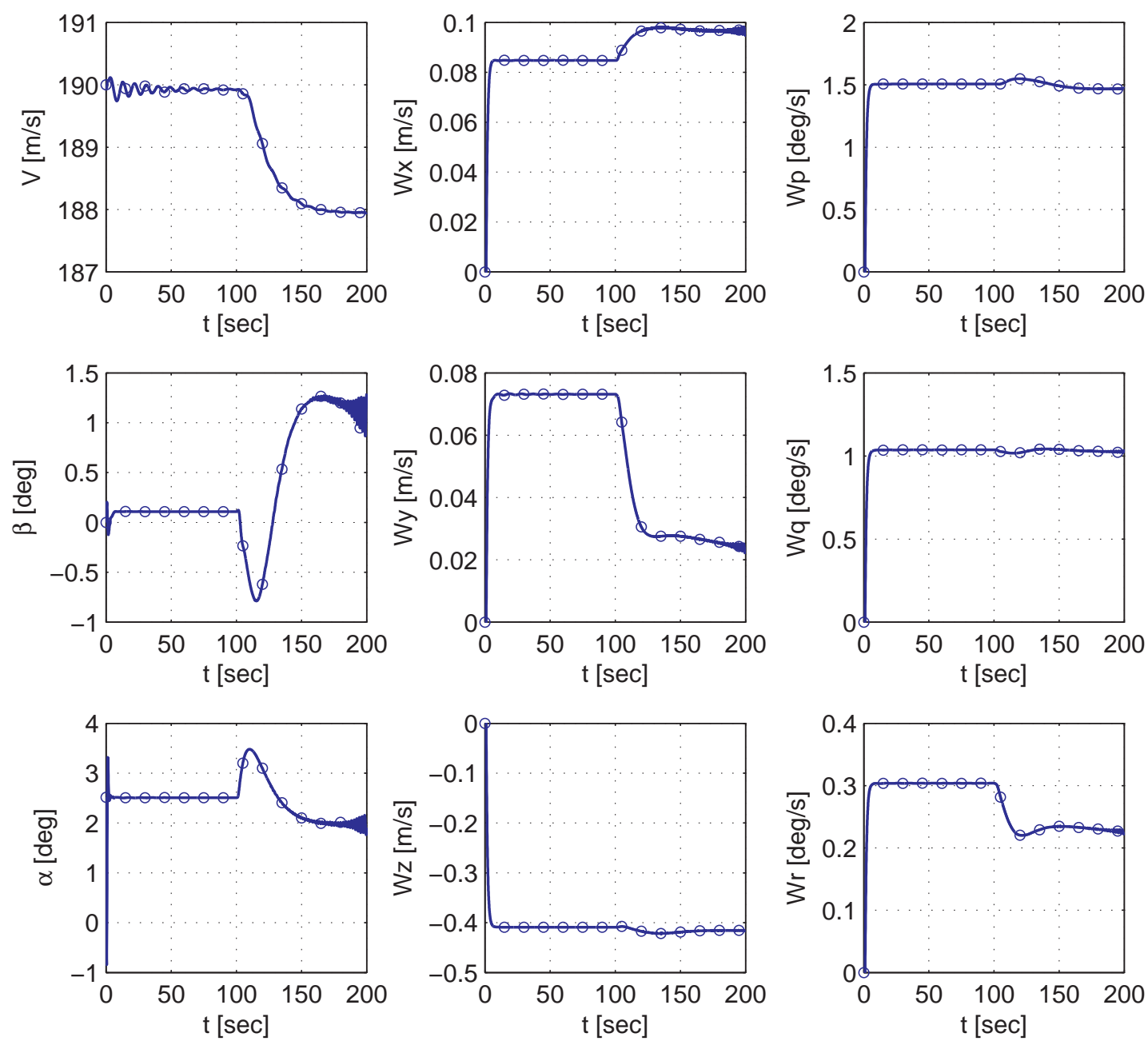

Figure E.22. Revised Weight Q, R: $V, \beta, \alpha$, and Winds for Nonlinear Simulation (Interactive Flow Field Only) LQR MIMO + Friedland Control Disturbance Rejection while Tanker Performs a 30 Degree Bank. 

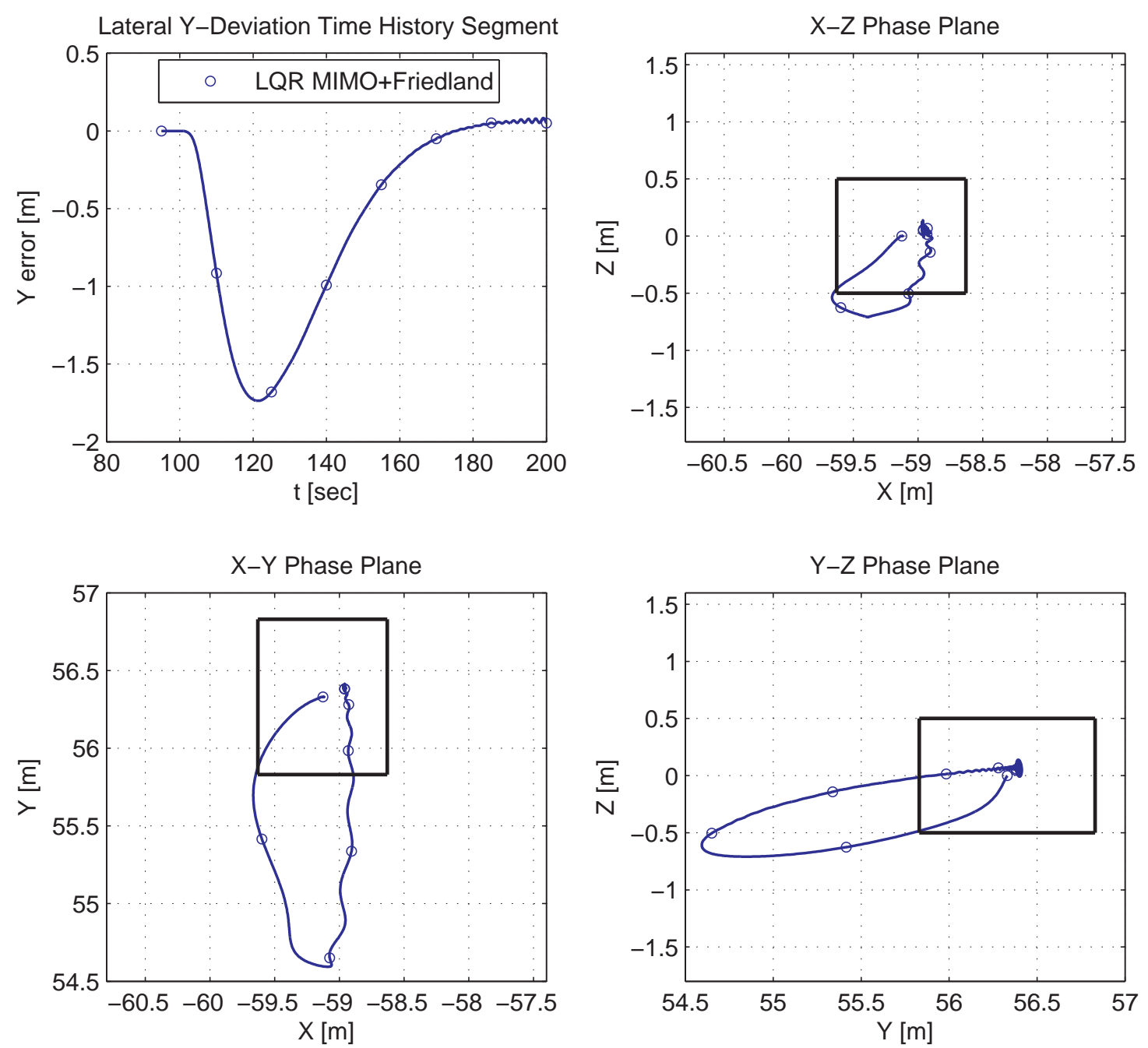

Figure E.23. Revised Weight Q, R: Phase Plane LQR MIMO + Friedland Control Only for Nonlinear Simulation (Interactive Flow Field Only) while Tanker Performs a 30 Degree Bank. 
E.2.2 Results for Observation Relative Position Station-Keeping in the Presence of Tanker Flow Field and Non-Steady Prevailing Wind

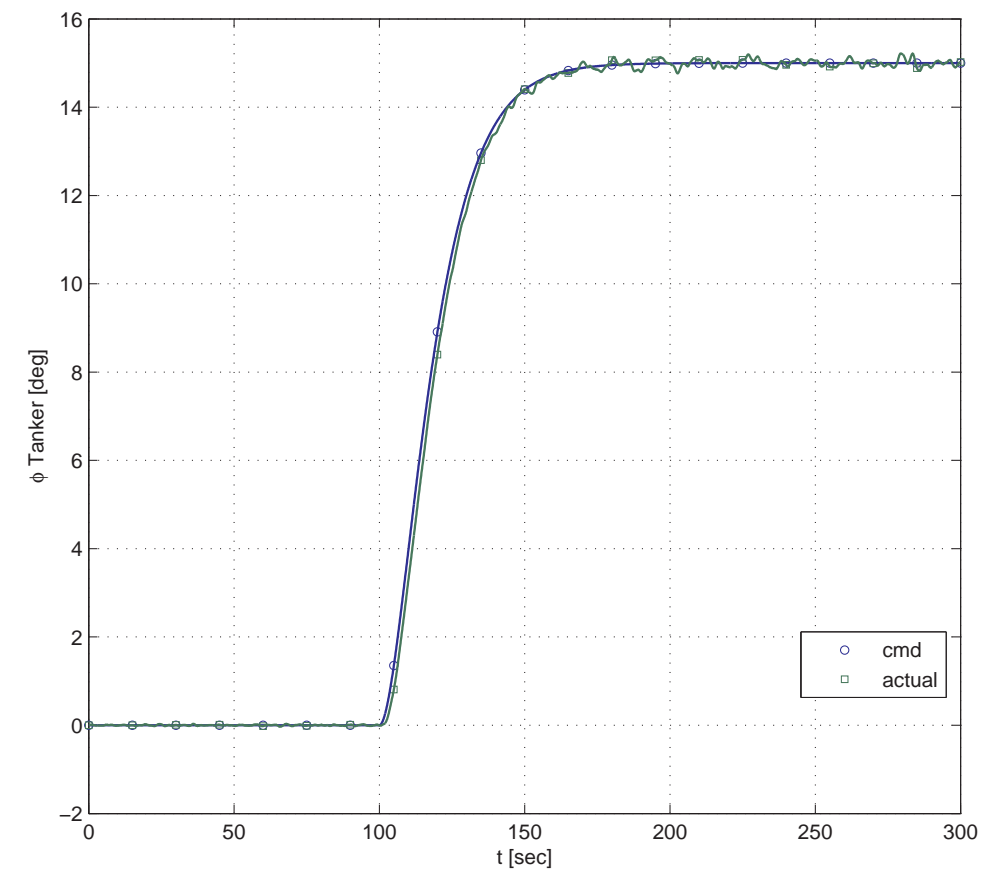

Figure E.24. Nonlinear Simulation Tanker Performing a 15 Degree Right Bank in NonSteady Atmosphere. 


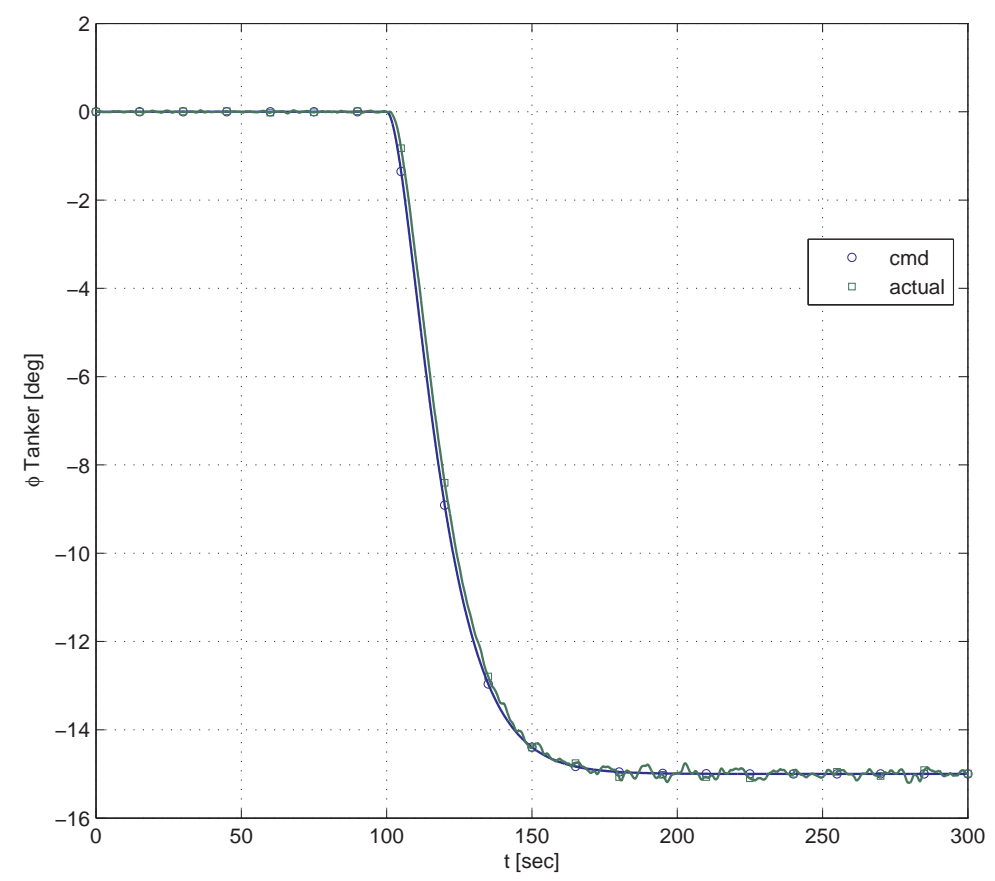

Figure E.25. Nonlinear Simulation Tanker Performing a -15 Degree Left Bank in NonSteady Atmosphere. 

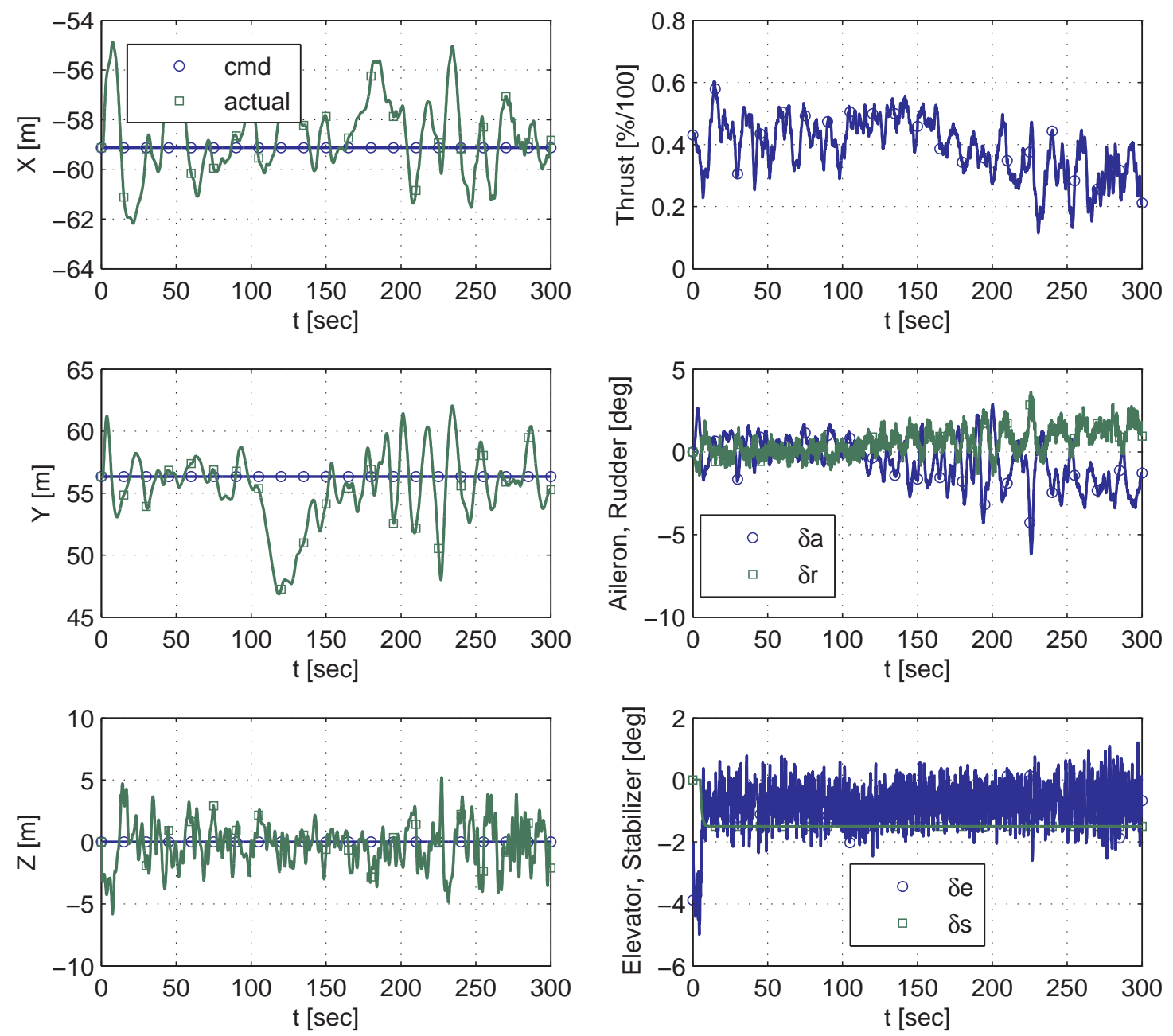

Figure E.26. Currently Employed Q, R: Station-Keeping and Effectors for Nonlinear Simulation LQR MIMO Control while Tanker Performs a 15 Degree Right Bank in NonSteady Atmosphere. 

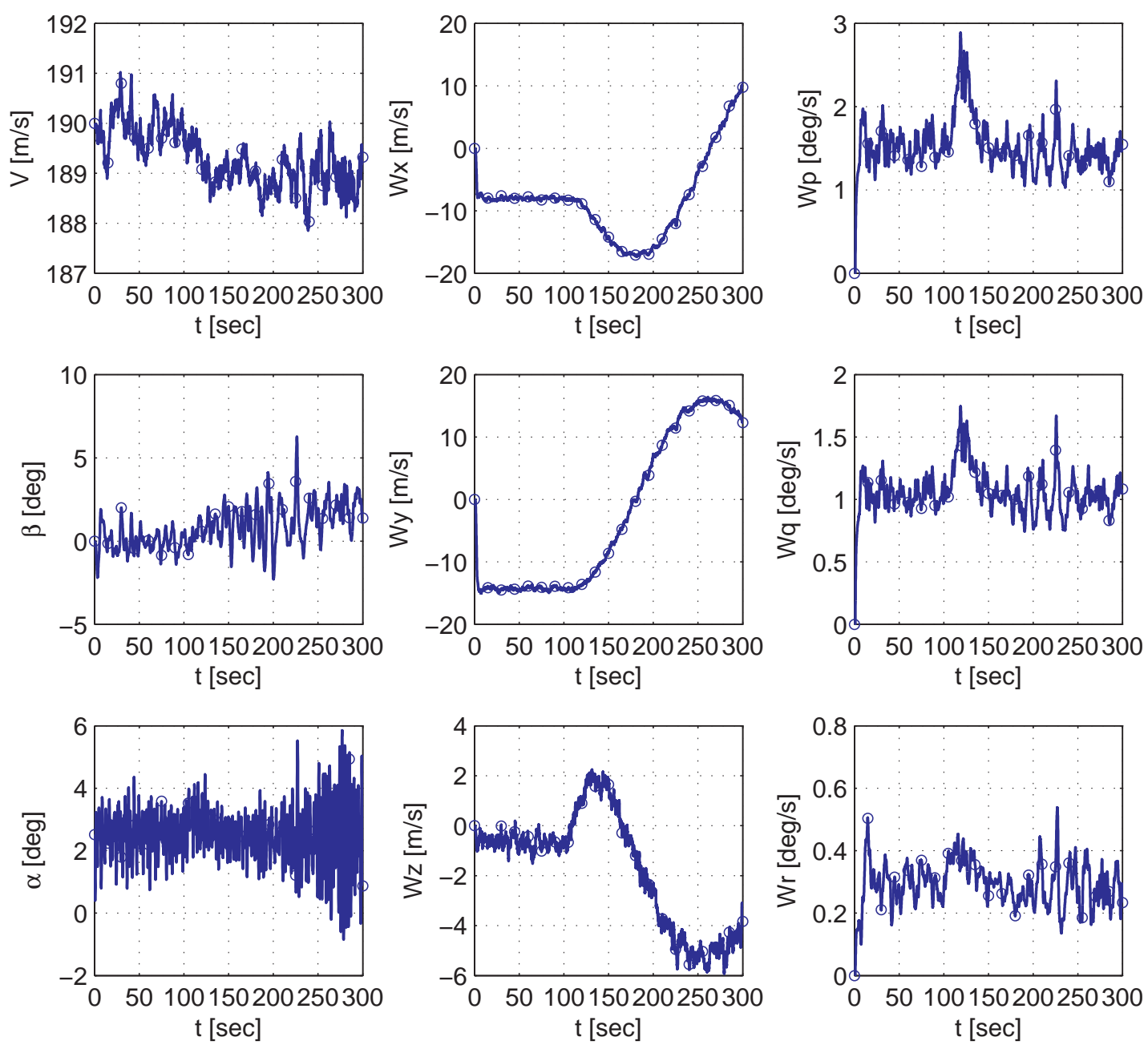

Figure E.27. Currently Employed Q, R: $V, \beta, \alpha$, and Winds for Nonlinear Simulation LQR MIMO Control while Tanker Performs a 15 Degree Right Bank in Non-Steady Atmosphere. 

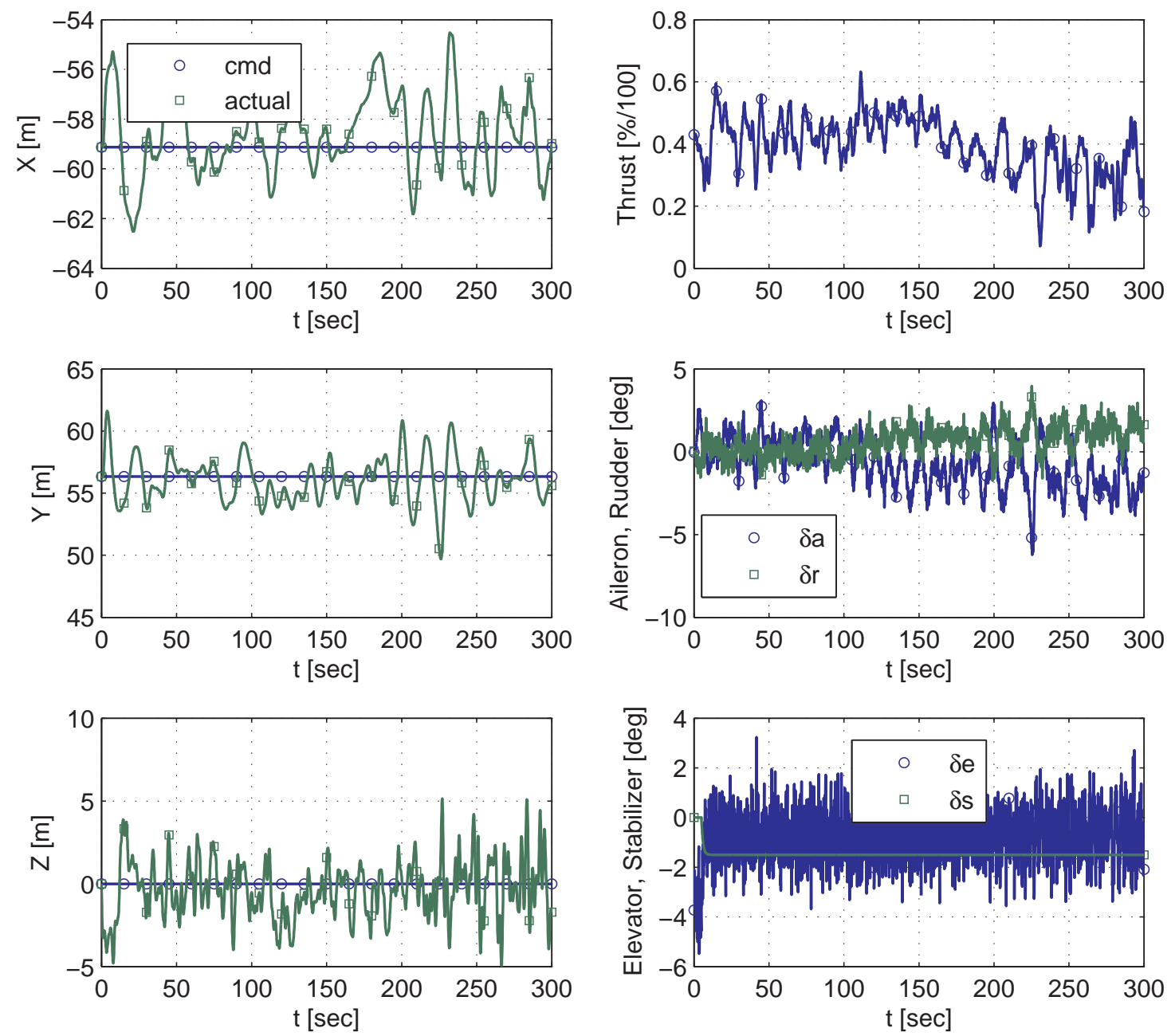

Figure E.28. Currently Employed Q, R: Station-Keeping and Effectors for Nonlinear Simulation LQR MIMO + Friedland Control while Tanker Performs a 15 Degree Right Bank in Non-Steady Atmosphere. 

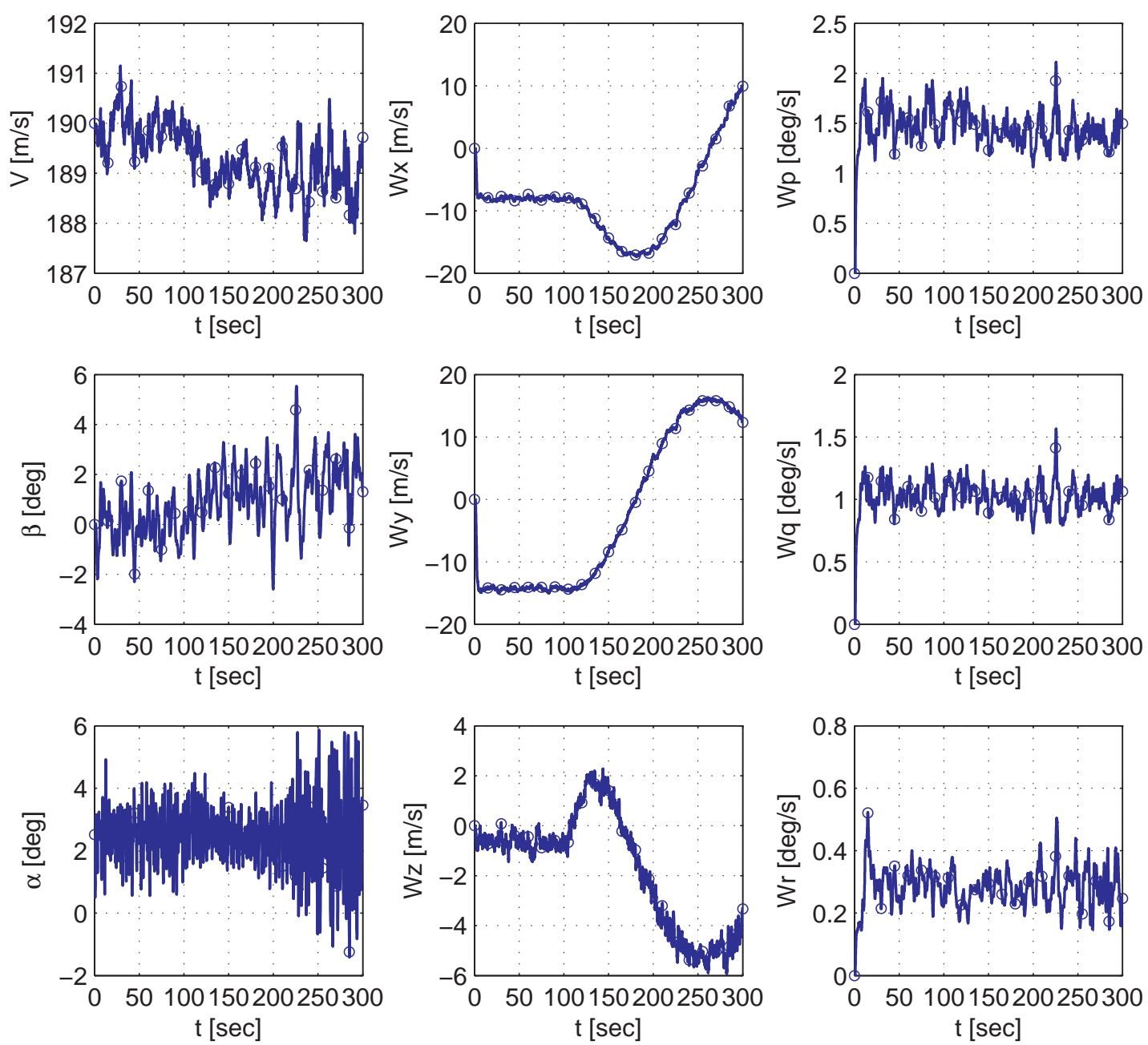

Figure E.29. Currently Employed Q, R: $V, \beta, \alpha$, and Winds for Nonlinear Simulation LQR MIMO + Friedland Control while Tanker Performs a 15 Degree Right Bank in Non-Steady Atmosphere. 

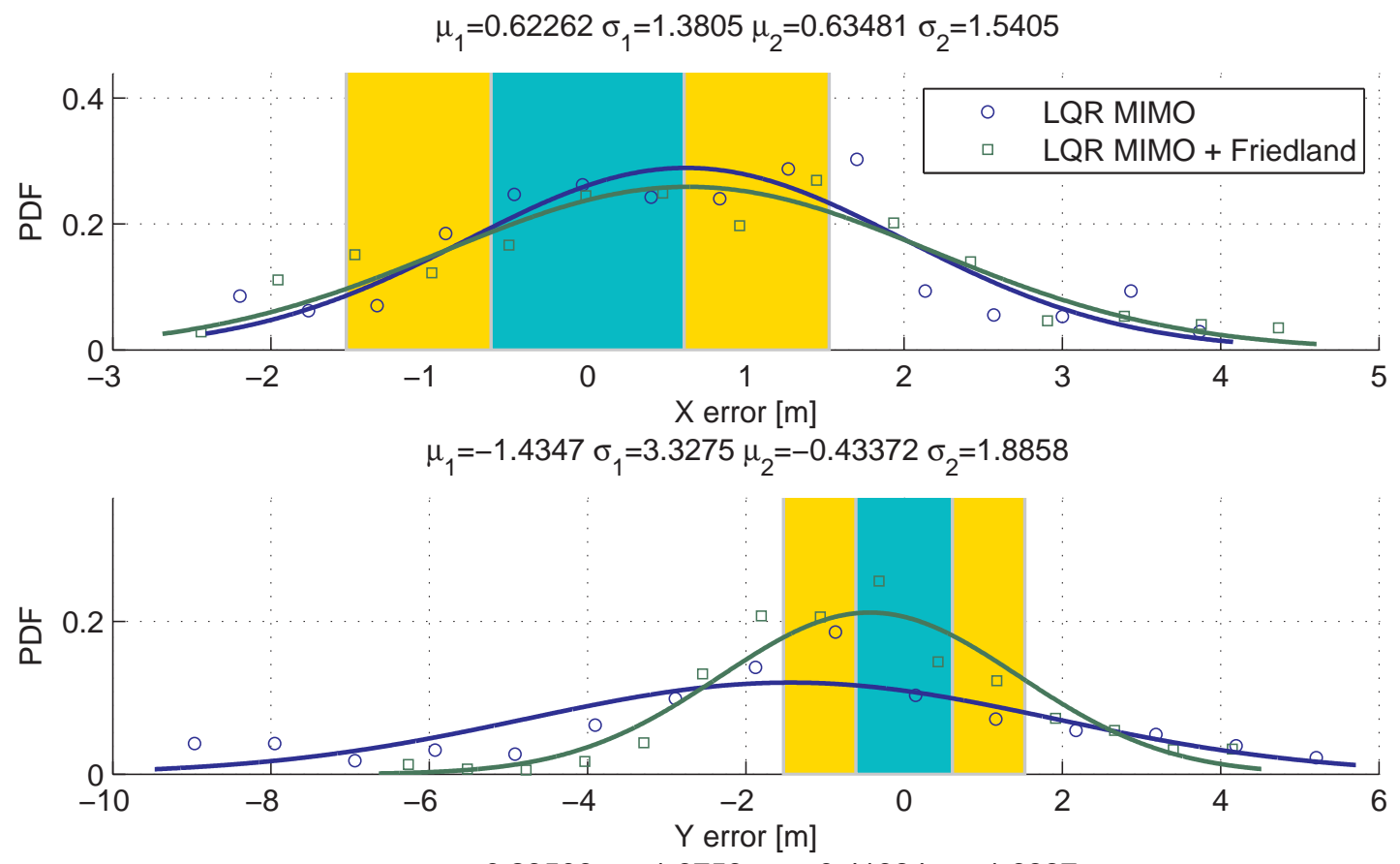

$\mu_{1}=-0.23506 \sigma_{1}=1.6753 \mu_{2}=-0.41384 \sigma_{2}=1.6887$

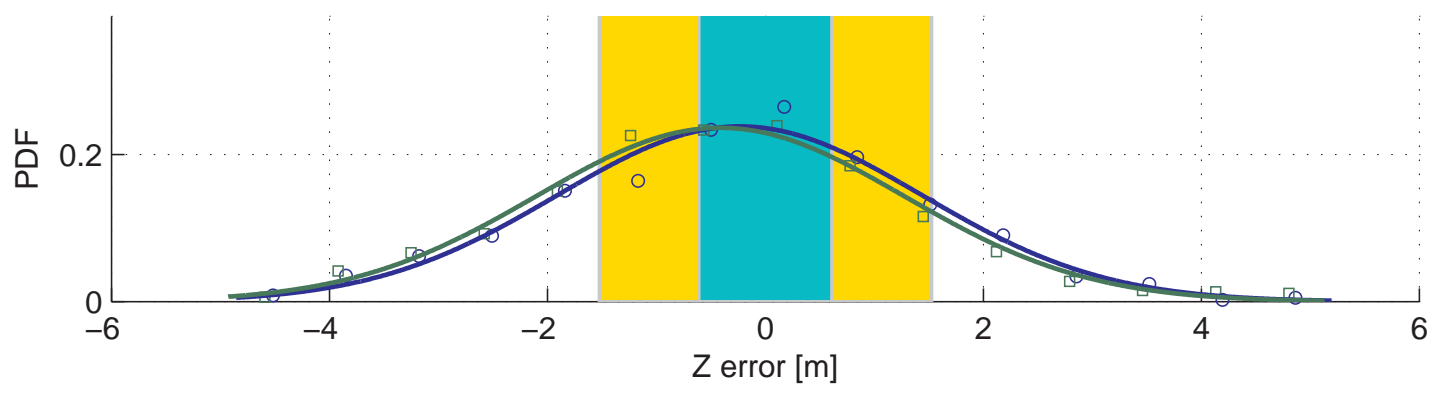

Figure E.30. Currently Employed Q, R: Gaussian Probability Density Function Controller Comparison for Nonlinear Simulation while Tanker Performs a 15 Degree Right Bank in Non-Steady Atmosphere. 

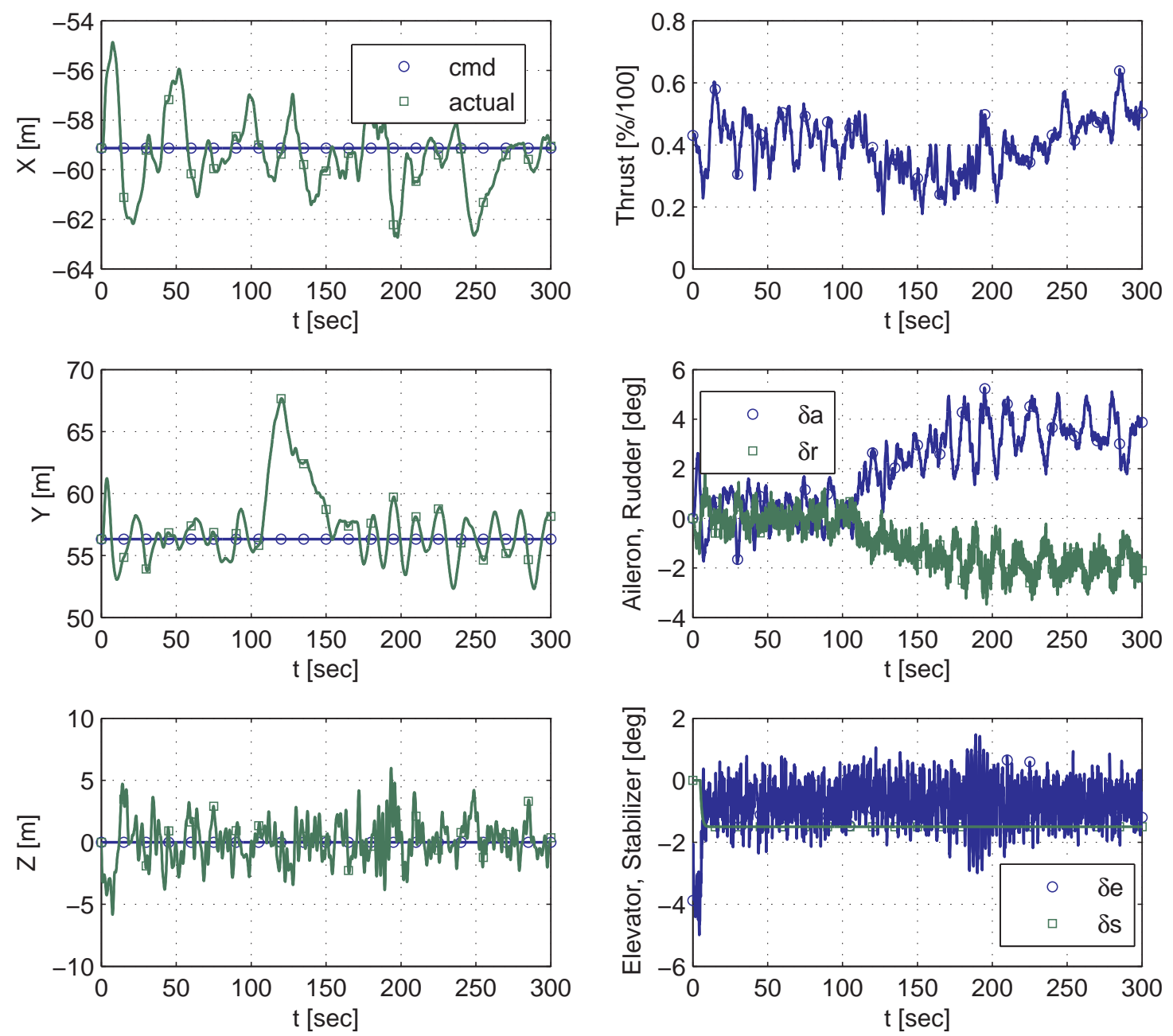

Figure E.31. Currently Employed Q, R: Station-Keeping and Effectors for Nonlinear Simulation LQR MIMO Control while Tanker Performs a -15 Degree Left Bank in NonSteady Atmosphere. 

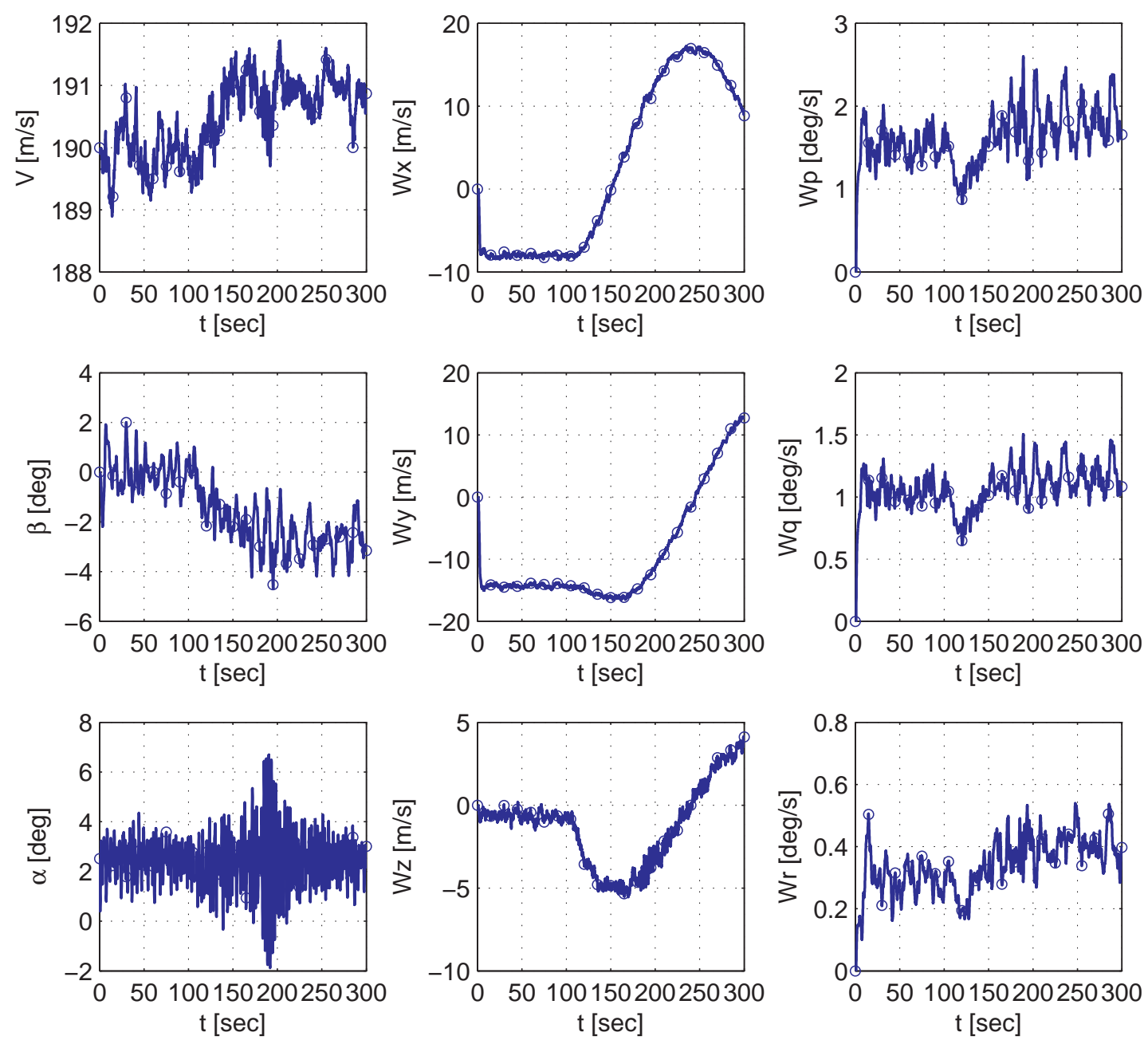

Figure E.32. Currently Employed Q, R: $V, \beta, \alpha$, and Winds for Nonlinear Simulation LQR MIMO Control while Tanker Performs a -15 Degree Left Bank in Non-Steady Atmosphere. 

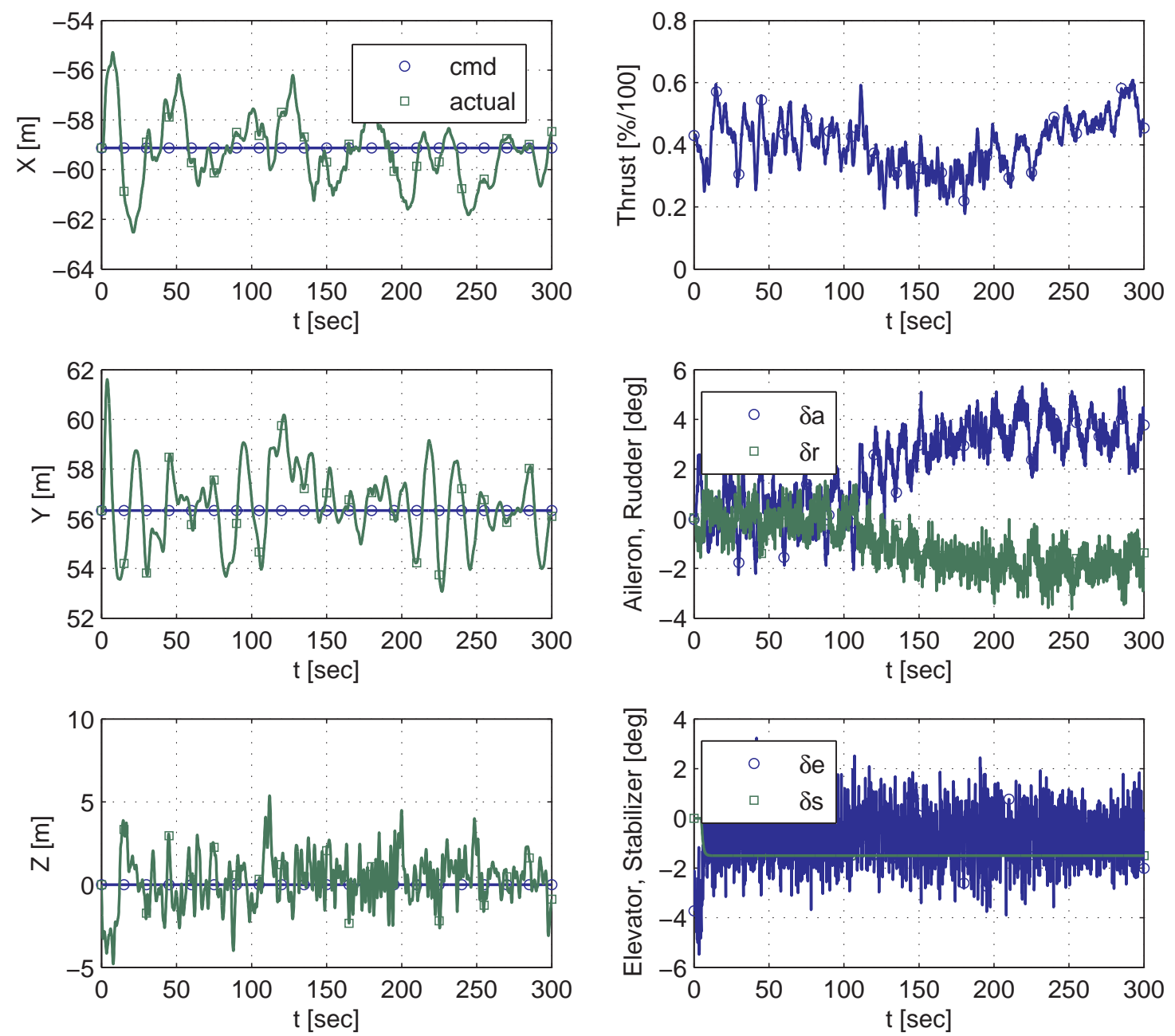

Figure E.33. Currently Employed Q, R: Station-Keeping and Effectors for Nonlinear Simulation LQR MIMO + Friedland Control while Tanker Performs a -15 Degree Left Bank in Non-Steady Atmosphere. 

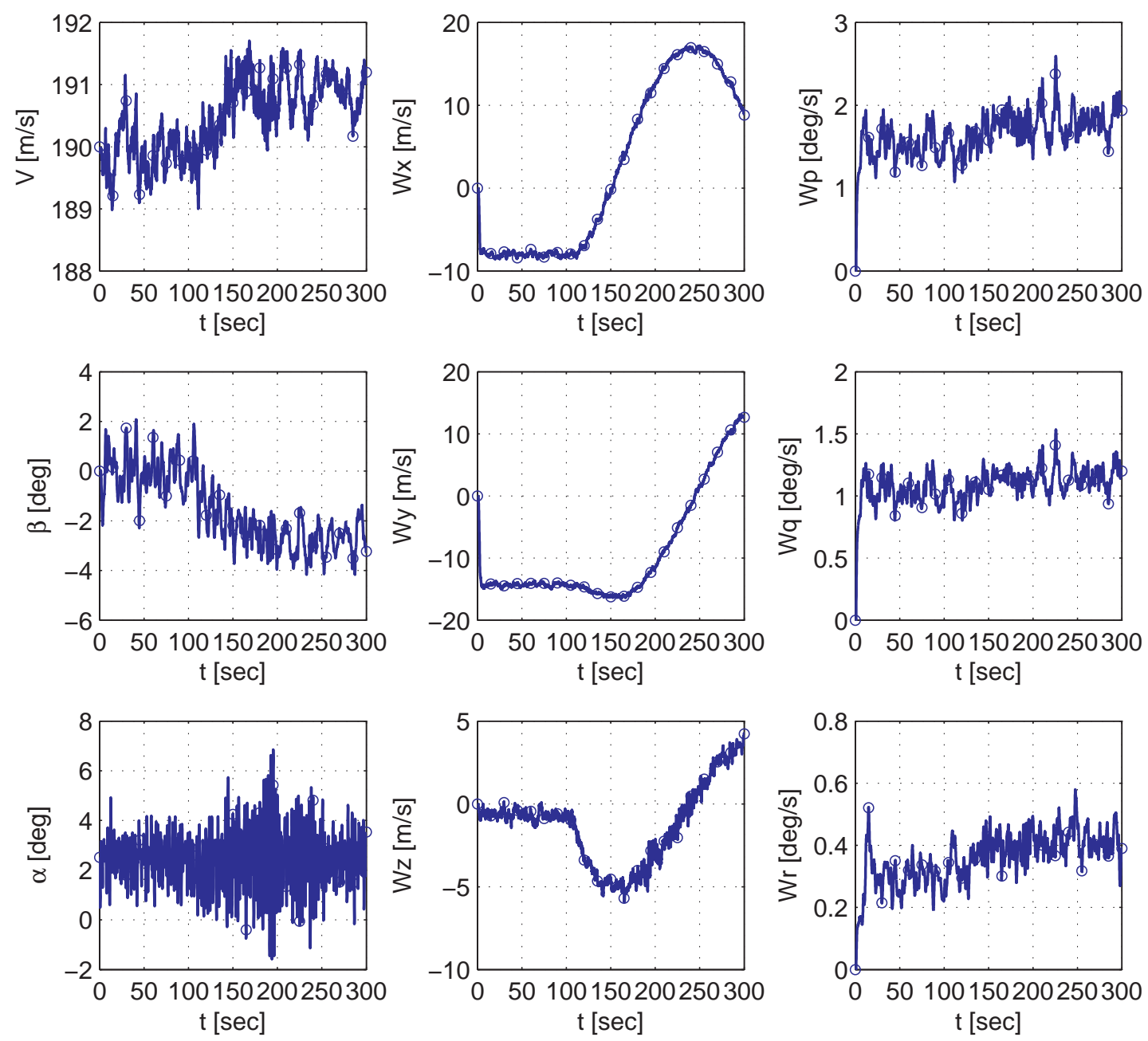

Figure E.34. Currently Employed Q, R: $V, \beta, \alpha$, and Winds for Nonlinear Simulation LQR MIMO + Friedland Control while Tanker Performs a -15 Degree Left Bank in Non-Steady Atmosphere. 

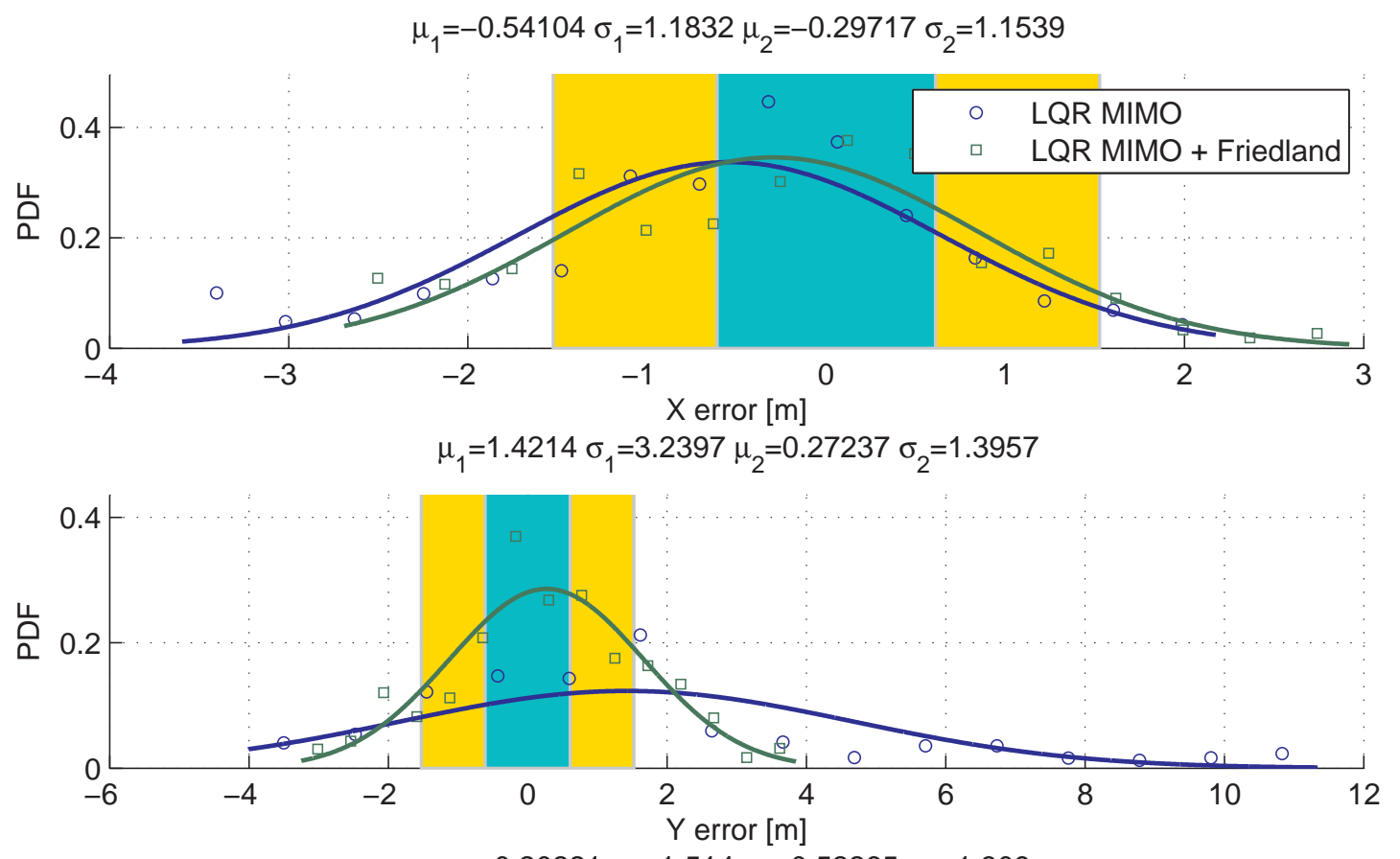

$\mu_{1}=0.20221 \sigma_{1}=1.514 \mu_{2}=0.52265 \sigma_{2}=1.309$

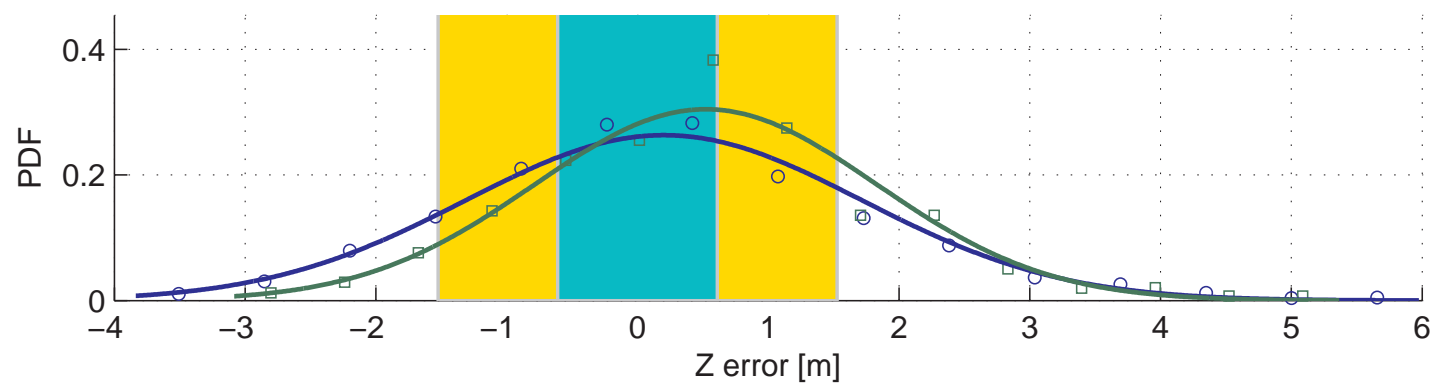

Figure E.35. Currently Employed Q, R: Gaussian Probability Density Function Controller Comparison for Nonlinear Simulation while Tanker Performs a -15 Degree Left Bank in Non-Steady Atmosphere. 


\section{REFERENCES}

[1] E. Kim, Control and Simulation of Relative Motion for Aerial Refueling in Racetrack Maneuver. Arlington, TX: MSAE thesis, The University of Texas at Arlington, May 2007.

[2] Wikipedia, "Aerial refueling," in http://en.wikipedia.org/wiki/Aerial_refueling, 2009.

[3] C. Elliott and A. Dogan, "Improving receiver station-keeping in aerial refueling by formulating tanker motion as disturbance," in Proceedings of AIAA Atmospheric Flight Mechanics Conference, Chicago, IL, August 2009, AIAA paper 2009-5602.

[4] C. Elliott, A. Dogan, and W. Blake, "Effects of mass and size on control of large receiver in aerial refueling," in Proceedings of AIAA Atmospheric Flight Mechanics Conference, Chicago, IL, August 2009, AIAA paper 2009-5927.

[5] J. Tucker, "Derivation of the dynamics equations of receiver aircraft in aerial refueling," Master's thesis, The University of Texas at Arlington, Arlington, TX, December 2007.

[6] J. Tucker, A. Dogan, and W. Blake, "Derivation of the dynamics equations of receiver aircraft in aerial refueling," in press for publication in AIAA Journal of Guidance, Control and Dynamics, 2009.

[7] _ _ , "Derivation of the dynamics equations of receiver aircraft in aerial refueling," in Proceedings of the AIAA 45th AIAA Aerospace Sciences Meeting and Exhibit, Reno, NV, August 2007, AIAA paper 2007-251. 
[8] J. Waishek, Derivation of the Dynamics Equations for Receiver Aircraft in Aerial Refueling. Arlington, TX: MSAE thesis, The University of Texas at Arlington, December 2007.

[9] E. Kim, A. Dogan, and W. Blake, "Control of a receiver aircraft relative to the tanker in racetrack maneuver," in Proceedings of the AIAA Guidance, Navigation, and Control Conference, Keystone, CO, August 2006, AIAA paper 2006-6710.

[10] A. Dogan, E. Kim, and W. Blake, "Control and simulation of relative motion for aerial refueling in racetrack maneuvers," Journal of Guidance, Control and Dynamics, vol. 30, no. 5, pp. 1551-1557, 2007.

[11] A. Dogan, S. Sato, and W. Blake, "Flight control and simulation for aerial refueling," in Proceedings of AIAA Guidance, Navigation, and Control Conference, San Francisco, CA, August 2005, AIAA paper 2005-6264.

[12] A. Dogan, S. Venkataramanan, and W. Blake, "Modeling of aerodynamic coupling between aircraft in close proximity," Journal of Aircraft, vol. 42:4, pp. 941-955, 2005.

[13] S. Venkataramanan and A. Dogan, "Modeling of aerodynamic coupling between aircraft in close proximities," in Proceedings of the AIAA Atmospheric Flight Mechanics Conference and Exhibit, Providence, RI, August 2004, AIAA paper 2004-5172.

[14] — - "Dynamic effects of trailing vortex with turbulence \& time-varying inertia in aerial refueling," in Proceedings of the AIAA Atmospheric Flight Mechanics Conference and Exhibit, Providence, RI, August 2004, AIAA paper 2004-4945.

[15] S. Venkataramanan, A. Dogan, and W. Blake, "Vortex effect modelling in aircraft formation flight," in Proceedings of the AIAA Atmospheric Flight Mechanics Conference and Exhibit, Austin, TX, August 2003, AIAA paper 2003-5385. 
[16] T. A. Lewis, Flight Data Analysis and Simulation of Wind Effects During Aerial Refueling. Arlington, TX: MSAE thesis, The University of Texas at Arlington, May 2008.

[17] A. Dogan, T. Lewis, and W. Blake, "Wake-vortex induced wind with turbulence in aerial refueling - part a: Flight data analysis," in Proceedings of the AIAA Atmospheric Flight Mechanics Conference and Exhibit, Honolulu, Hawaii, August 2008, AIAA-2008-6696.

[18] — - "Wake-vortex induced wind with turbulence in aerial refueling - part b: Model and simulation validation," in Proceedings of the AIAA Atmospheric Flight Mechanics Conference and Exhibit, Honolulu, Hawaii, August 2008, AIAA-2008-6697.

[19] _ - "Flight data analysis and simulation of wind effects during aerial refueling," AIAA Journal of Aircraft, vol. 45, no. 6, pp. 2036-2048, November-December 2008.

[20] B. Friedland, Control System Design, An Introduction to State-Space Methods. New York, NY: McGraw-Hill, Inc., 1986, ch. 10.

[21] C. Johnson, "Accommodation of external disturbances in linear regulator and servomechanism problems," in IEEE Transactions on Automatic Control, vol. AC-16, no. 6,1971 , pp. $635-644$.

[22] J. Profeta, W. Vogt, and M. Mickle, "Disturbance estimation and compensation in linear systems," in IEEE Transaction on Aerospace and Electronic Systems, vol. 26, no. 2, 1990, pp. 225-231.

[23] C. Johnson, "Adaptive controller design using disturbance-accommodation techniques," in International Journal of Control, vol. 42, 1985, pp. 193-210.

[24] J. Han, "Auto-disturbance rejection control and its applications," vol. 13, no. 1, 1998, pp. 19-23. 
[25] Q. Zheng, "On active disturbance rejection control: Stability analysis and applications in disturbance decoupling control," Ph.D. dissertation, Cleveland State University, Cleveland, OH, 2009.

[26] B. L. Stevens and F. L. Lewis, Aircraft Control and Simulation, 2nd Edition. USA: John Wiley \& Sons, Inc., 2003.

[27] F. L. Lewis and V. L. Syrmos, Optimal Control. USA: John Wiley \& Sons, Inc., 1995.

[28] J. Bryson, A. E. and Y. C. Ho, Applied Optimal Control. USA: New York: Hemisphere, 1975. 


\section{BIOGRAPHICAL STATEMENT}

Christopher Michael Elliott was born in Houston, Texas on July 11, 1976. He received a Bachelor of Science degree in Aerospace Engineering from the University of Texas at Austin in December 1998. Concurrent with undergraduate studies, he worked at the Institute of Advanced Technology as a Research Engineer in the Electro-Magnetic Launch Projectiles (EMLP) Dynamics group. After graduation, he worked as a Software Engineer Consultant with the Radx Corporation out of Plano, Texas until accepting a position in October 1999 with the United Space Alliance as an Aerospace Engineer in the International Space Station Program Office at the Johnson Space Center in Houston, Texas. Transferring to the Automation and Robotics NASA directorate in August 2000 with Lockheed Martin Space Operations, he worked in the Multi-use Remote Manipulator Development Facility (MRMDF) until February 2003. During this period, Chris began graduate studies at the University of Houston at Clear Lake in Software Engineering. In February 2003, he transferred to Lockheed Martin Aeronautics Company in Fort Worth, Texas and joined the F16 Control Law Analysis and Design Flight Dynamics team. Along with the career change from the space industry to the atmospheric flight industry, Chris began a new graduate degree path at the University of Texas at Arlington in Aerospace Engineering with an emphasis on Flight Mechanics in the Spring of 2006. Today he continues full time employment with the Lockheed Martin Aeronautics Company with the Flight Dynamics team for the F16, F2, and T50 aircraft as well as the ADP Skunkworks Flight Controls team. He has published multiple technical papers at

the American Institute of Aeronautics and Astronautics (AIAA) as a senior professional 
member and plans to continue postgraduate research with Dr. Atilla Dogan subsequent to a MS graduation in December 2009. 